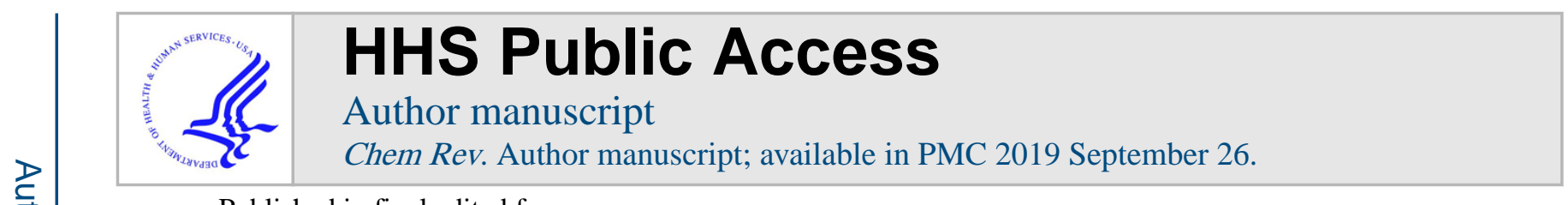

Published in final edited form as:

Chem Rev. 2018 September 26; 118(18): 8798-8888. doi:10.1021/acs.chemrev.7b00698.

\title{
Redox Signaling by Reactive Electrophiles and Oxidants
}

\author{
Saba Parvez ${ }^{\S, \dagger, \uparrow, ~ M a r c u s ~ J . ~ C . ~ L o n g ~}{ }^{\dagger, \uparrow}$, Jesse R. Poganik ${ }^{\ddagger, \dagger}$, and Yimon Aye ${ }^{\ddagger, \dagger, ¥,{ }^{\star}}$ \\ ‡Ecole Polytechnique Fédérale de Lausanne, Institute of Chemical Sciences and Engineering, \\ 1015, Lausanne, Switzerland §Department of Pharmacology and Toxicology, College of \\ Pharmacy, University of Utah, Salt Lake City, Utah, 84112, USA tDepartment of Chemistry and \\ Chemical Biology, Cornell University, Ithaca, New York, 14853, USA ${ }^{*}$ Department of Biochemistry, \\ Weill Cornell Medicine, New York, New York, 10065, USA
}

\begin{abstract}
The concept of cell signaling in the context of non-enzyme-assisted protein modifications by reactive electrophilic and oxidative species—broadly known as redox signaling —is a uniquely complex topic that has been approached from numerous different and multidisciplinary angles. Our Review reflects on 5 aspects critical for understanding how Nature harnesses these noncanonical post-translational modifications to coordinate distinct cellular activities: (1) specific players and their generation; (2) physicochemical properties; (3) mechanisms of action; (4) methods of interrogation; and (5) functional roles in health and disease. Emphasis is primarily placed on the latest progress in the field, but several aspects of classical work likely forgotten/lost are also recollected. For researchers with interests in getting into the field, our Review is anticipated to function as a primer. For the expert, we aim to stimulate thought and discussion about fundamentals of redox signaling mechanisms, and nuances of specificity/selectivity and timing in this sophisticated yet fascinating arena at the crossroads of chemistry and biology.
\end{abstract}

\section{Graphical Abstract}

Correspondence: ya222@ cornell.edu (Y.A.).

ItEqual contributions

A patent application related to the content of the Review has been filed by Cornell University 


\section{INTRODUCTION}

The cell is a microcosm of the global compartmentalized multimedia information superhighway. Signals constantly flow hither and thither mediating information interchange between proteins/pathways/organelles as well as translating coded extracellular information to chemical signals that form the language of the cell. Unsurprisingly, a complex series of signaling mechanisms have evolved: these are big business for the cell in terms of the resources they use and the benefits they can bring. Since these signaling processes control specific decision making, they are intrinsically linked to both etiology and treatment of disease. They have thus also become cornerstones of drug discovery and design.

Chemical signals are typically considered to be relayed by specific enzymes. The two principal enzyme-orchestrated signaling codes are phosphate and ubiquitin/ubiquitin-like modifiers. Enzymes catalyzing these information interchanges/relays account for around $10 \%$ of the genome [roughly 1000 genes for each pathway; a number similar to the number of genes required by mitochondria(1)]. This conjugation machinery faithfully and efficiently identifies specific upstream signals, writes appropriate coded information that downstream proteins can interpret and hence ultimately ensures a specific response occurs to a particular stimulus at a specific time. This guild of writers, readers, and erasers is required because a single chemical signal, depending on context can usher a huge number of different downstream signaling processes. Thus, the key to these pathways is the specificity and precision ensured by the enzymes that orchestrate them and the regulation that these processes impart.

However, there is a second signaling mode lacking the polished syntax and structure of enzymatic signaling cascades. This vernacular is conveyed through inherently reactive chemical signals that modify their specific target proteins largely without enzyme mediation. This is the focus of our review. We focus specifically on reactive oxidants and electrophiles. Reactive chemical signals are similar to classical methyl/acetyl/malonyl/phosphate/ubiquitin signals in that they modulate signaling pathways in a context specific manner. However, their chemical AND functional promiscuity renders them a potential source of misinformation rather than a means to precisely coordinate responses to promote fitness. Thus, at first glance, signaling by reactive chemicals is distinct from traditional ordered, logical signaling pathways. It seems the cell would have no need for chemically inefficient and promiscuous modifications. Nonetheless, there is growing evidence that many reactive oxidative and electrophilic species (ROS/RES) possess the ability to act as cellular signals. Consistent with this argument, many proteins have evolved to be sensitive to specific forms of these signals, and to further resist overreaction once a specific signaling pathway has been modified. Moreover, such protein-specific non-enzymatic modifications at low occupancy elicit specific signaling outputs just like in enzyme-orchestrated signaling pathways.

In this review, we will introduce the main players in ROS/RES signaling in eukaryotic cells. We will then set out the physicochemical properties of biological signals and sensor proteins that allow them to act as specific sensor/signal pairs in the backdrop of the cellular milieu. Because these signals must find their target in the cell ostensibly unaided, we will discuss important dimensions and varied local/regional concentrations of reactive species within the 
cell and organelles. Based on these properties, we will then critically review and evaluate specific examples of these signal sensors and the pathways they modulate in the context of physiologically-relevant biological signaling processes. We will next discuss methods to interrogate redox signaling and evaluate relevance of redox signaling as a new paradigm in therapeutic interventions through the discussions of key druggable proteins and how these proteins can be harvested for therapies. Our two-pronged goal is to provide a new lens to understand both redox signaling as a physiological signaling prototype, and its emerging translational impacts on human medicine.

\section{KEY PLAYERS IN NON-ENZYME-MEDIATED CELL SIGNALING}

A large number of reactive intermediates are generated during normal physiological and pathological conditions;(2) for instance there are 70,000 DNA lesions formed by chemical modification of DNA per cell per day in humans.(3) However, recent research indicates the nuanced ability of biological systems to harness ROS/RES—once considered only to play damaging roles-as small-molecule signaling mediators essential for fitness.

ROS and RES are two major categories of reactive redox-linked signals. Other reactive species exist in the cell, including reactive nitrogen and sulfur species (RNS/RSS). Many of the conditions that generate ROS/RES can also generate RNS/RSS, so these species are often present together. However, physical properties of individual subtypes, identity of specific sensor protein targets, and/or downstream signaling elicited by these species are likely different. Accordingly, reactive species can be reasonably subcategorized and considered separately. We will only discuss RES and ROS; we direct readers to complementary reviews on RNS(4-6) and RSS.(5,7-9)

Most ROS are generated as a result of reduction of molecular oxygen.(10) ROS react with various proteins and other small molecules, resulting in the generation of a wide variety of secondary redox signals including RES. RES are also generated by the action of enzymatic pathways such as cyclooxygenases (COXs) $(11,12)$ and lipoxygenases (LOXs)(13-15) and as byproducts of some metabolic processes.(16) This section describes the biogenesis of ROS and RES and highlights the key cellular compartments and processes responsible for their generation.

\subsection{Reactive oxygen species (ROS)-mediated signal transduction}

2.1.1 Physiologically-relevant oxidants $-\mathrm{E}^{0}$ is the most common parameter to characterize the "power" of an oxidant $\left(\Delta \mathrm{G}=-\mathrm{nFE}^{0}\right.$, where $\mathrm{n}$ and $\mathrm{F}$ are number of electrons transferred and the Faraday constant, respectively). Thus, for a molecule that is prone to behave as an oxidant, reduction potential is positive. However, it is worth noting that redox exchange requires two players, and so long as there is a significant difference between redox potentials, redox exchange can occur [regardless of the sign(s) of the respective $\mathrm{E}^{0} \mathrm{~s}$ ]. It is worth remembering that $\mathrm{E}^{0}$ defines thermodynamic behavior. Thus, the effective reduction potential has to be scaled to account for the relative concentrations of each component of the equilibrium: $E=E^{0}-\frac{R T}{n F} \ln \left[\frac{\text { reduced }}{\text { oxidized }}\right]$. Furthermore, in the absence of enzymatic assistance, chemoselectivity may be dictated more by kinetics/frontier-molecular-orbital (FMO) 
interactions than thermodynamics. One interesting example of this phenomenon is nucleophilic vs. electrophilic oxidants. Substrates bearing both thioether and sulfoxide functional groups, such as thianthrene-5-oxide, are regioselectively oxidized by electrophilic oxidants like m-CPBA at the thioether, the more electron-rich moiety. On the other hand, thianthrene-5-oxide is oxidized by anionic oxidants, such as m-CPBA/NaOH, at the sulfoxide, the more electron-deficient moiety, to give the sulfone (Figure S1).

Regiodivergence can be explained by the fact that the higher HOMO (S lone pair of thioether) is best matched to overlap with the $\sigma^{*}(\mathrm{O}-\mathrm{O})$ orbital in the protonated peracid. However, once the peracid is deprotonated, the thiol lone pair is repelled suppressing nucleophilic attack at the $\sigma^{*}(\mathrm{O}-\mathrm{O})$. However, the negatively-charged peracid $\mathrm{O}$ lone pairs are raised in energy due to electron pair repulsion, allowing good overlap with the sulfoxide $\pi^{*}$ orbital. Instances where these phenomena may impact biology are discussed below.

Principal cellular ROS include radicals [e.g., superoxide $\left(\mathrm{O}_{2}{ }^{--}\right)$and hydroxyl radical $\left({ }^{\circ} \mathrm{OH}\right)$ ], and non-radical species [e.g., hydrogen peroxide $\left(\mathrm{H}_{2} \mathrm{O}_{2}\right)$ and singlet oxygen $\left({ }^{1} \mathrm{O}_{2}\right)$ ]. (17) $\mathrm{O}_{2}{ }^{--}\left[\mathrm{E}^{0}\left(\mathrm{O}_{2}{ }^{-}-/ \mathrm{H}_{2} \mathrm{O}_{2}\right)=0.9 \mathrm{~V}(18)\right]$ is produced by the single electron reduction of molecular oxygen with reductants such as NADPH and $\mathrm{FADH}_{2}$ acting as the electron donors (see section 2.1.2). $\mathrm{O}_{2}{ }^{-}$-is the primary oxidant generated within many organelles. Enzymes responsible for this process include NADPH oxidases (NOX) that are located at the plasma membrane and the electron transport chain (ETC) in the mitochondria. Nevertheless, $\mathrm{O}_{2}$ - -steady-state concentration is maintained at picomolar levels due to its enzyme-catalyzed dismutation [by superoxide dismutase (SOD)] to $\mathrm{H}_{2} \mathrm{O}_{2}$ and oxygen.(19) In addition to SOD, an emerging class of membrane-bound enzymes termed superoxide oxidases (SOOs) oxidize $\mathrm{O}_{2}{ }^{-}$-to oxygen at diffusion-limited rates.(20) Despite its low abundance in mammalian cells, $\mathrm{O}_{2}{ }^{-}$-is important in the regulation of various cell signaling pathways. For instance, Angiotensin II-induced $\mathrm{O}_{2}{ }^{-}$-generation activates the p38-MAPK pathway resulting in altered gene expression in endothelial cells.(21) Generation of $\mathrm{O}_{2}{ }^{-}-$is also required for Angiotensin II-mediated regulation of vasopressin secretion in the central nervous system, thirst and salt appetite, and modulation of the sympathetic nervous system.(22)

Mitochondrial $\mathrm{O}_{2}{ }^{-}$-signal is critical in lifespan regulation in C. elegans, although the exact molecular mechanisms are unclear.(23)

$\mathrm{H}_{2} \mathrm{O}_{2}\left[\mathrm{E}^{0}\left(\mathrm{H}_{2} \mathrm{O}_{2} / \mathrm{H}_{2} \mathrm{O}\right)=1.35 \mathrm{~V}(18)\right]$ is the most abundant ROS in eukaryotes.(24) Dismutation of $\mathrm{O}_{2}{ }^{-}$-is a significant source of intracellular $\mathrm{H}_{2} \mathrm{O}_{2}$. Direct production of $\mathrm{H}_{2} \mathrm{O}_{2}$ by oxidases such as NOX4 [for which $90 \%$ of ROS produced is $\mathrm{H}_{2} \mathrm{O}_{2}$ and $\mathrm{O}_{2}{ }^{-}{ }^{-}$accounts for only $10 \%(25,26)$ ], xanthine $(27)$ and monoamine oxidases has also been reported.(27,28) The steady-state concentration of $\mathrm{H}_{2} \mathrm{O}_{2}$ ranges between 1-10 nM in cells (Table 1).(24) Deviations from this steady-state concentration trigger biological responses.(29) Typically, small and controlled changes are associated with signaling responses. The signaling role of $\mathrm{H}_{2} \mathrm{O}_{2}$ came to light with the discovery that treatment of human T cells with $\mathrm{H}_{2} \mathrm{O}_{2}$ activated NF- $\kappa B$ transcription factor.(30) N-acetyl cysteine (NAC) and other thiol compounds mitigated the effect of $\mathrm{H}_{2} \mathrm{O}_{2}$ induced NF- $\mathrm{kB}$ activation. NAC also blocked the activation of NF- $\kappa B$ upon stimulation with a diverse range of different stimuli including TNF- $\alpha$, phorbol esters, lipopolysaccharides, calcium ionophores, and double-stranded RNA, suggesting that NF- $\kappa \mathrm{B}$ activation is mediated by a common reactive signaling species.(30) Endogenouslygenerated $\mathrm{H}_{2} \mathrm{O}_{2}$ modulates a wide-range of signaling pathways mainly by oxidizing cysteine 
residues on protein targets such as kinases and phosphatases.(31,32) On the other hand, large perturbations in $\mathrm{H}_{2} \mathrm{O}_{2}$ concentrations induce stress response. Concentrations $>100 \mathrm{nM}$ can cause oxidative damage of biomolecules resulting in cell cycle arrest and eventual apoptosis. (24)

${ }^{1} \mathrm{O}_{2}$ \{formally, $\mathrm{O}_{2}\left({ }^{1} \Delta_{\mathrm{g}}\right) ;\left[\mathrm{E}^{0}\left({ }^{1} \mathrm{O}_{2} / \mathrm{O}_{2}{ }^{--}\right)=0.65 \mathrm{~V}(18)\right]$, excitation energy from ground state $\left.95 \mathrm{~kJ} \mathrm{~mol}^{-1}(33)\right\}$ is generated by the electronic excitation of triplet oxygen $\left\{{ }^{3} \mathrm{O}_{2}\right.$, more formally $\left.\mathrm{O}_{2}\left({ }^{3} \Sigma_{\mathrm{g}}{ }^{-}\right) ;\left[\mathrm{E}^{0}\left({ }^{3} \mathrm{O}_{2} / 2 \mathrm{H}_{2} \mathrm{O}\right)=0.82 \mathrm{~V}(34)\right]\right\}$. In biological systems, ${ }^{1} \mathrm{O}_{2}$ can be generated by (1) a light-induced excitation called the 'light reaction' and (2) a chemical excitation termed the 'dark reaction'.(35,36) In the light reaction, aromatic cellular constituents such as cytochromes and flavins as well as FAs(37) absorb light generating an excited triplet state $\left[\mathrm{T} 1\left(\pi \pi^{*}\right)\right]$ of the photosensitizer by intersystem crossing. The excited sensitizer forms an encounter complex with ${ }^{3} \mathrm{O}_{2}$, allowing the sensitizer to relax to a singlet state, generating reactive ${ }^{1} \mathrm{O}_{2}$ at the same time, in an overall spin-conserved process.(38) The dark reaction involves a chemical reaction, such as thermal decomposition of peroxynitrite $\left[\mathrm{ONOO}^{-} ; \mathrm{E}^{0}\left(\mathrm{ONOO}^{-} / \mathrm{NO}_{2}, \mathrm{H}_{2} \mathrm{O}\right)=1.4 \mathrm{~V}(39)\right]$ in the presence of peroxide (40) and enzymatic reactions catalyzed by myeloperoxidase, LOXs, and prostaglandin synthetases to generate ${ }^{1} \mathrm{O}_{2}$. (36) $\mathrm{O}_{2}$ formed in these processes is in the singlet state, due to spin conservation, since the starting materials are also singlet states (e.g., $\mathrm{HONO}_{2}$ ).(41) Because of the unique chemistry of ${ }^{1} \mathrm{O}_{2}$, it is able to perform two-electron oxidation chemistry - typically via ene and Diels-Alder reactions-and it is thus an effective oxidant of unsaturated lipids. In biological systems, generation of ${ }^{1} \mathrm{O}_{2}$ needs to be controlled because ${ }^{1} \mathrm{O}_{2}$ can oxidize several amino acid residues including cysteine, histidine, tyrosine, methionine and tryptophan.(42) Unsurprisingly, biology typically employs enzymes to generate highly localized bursts of ${ }^{1} \mathrm{O}_{2}$ at source that ultimately form less reactive signaling molecules, such as prostaglandins.(43) Uncontrolled release of ${ }^{1} \mathrm{O}_{2}$ induces oxidative damage. Oxidative damage to the eye and skin of animals has been attributed to UVmediated generation of ${ }^{1} \mathrm{O}_{2}$. (44) In fact, the ability of ${ }^{1} \mathrm{O}_{2}$ to cause excessive oxidative damage and apoptosis has led to its use as a photodynamic therapy for treating cancer.(42)

Another common biological oxidant is $\mathrm{HOCl}\left(\mathrm{E}^{0}=1.6 \mathrm{~V}\right)$, a molecule with a short (approx. $1 \mathrm{~min}$, although it is likely closer to seconds) half-life in cells. $(45,46)$ This molecule is involved in immune response as it is formed by the "myeloperoxidase- $\mathrm{H}_{2} \mathrm{O}_{2}-\mathrm{Cl}^{-}$" system in phagocytes (principally neutrophils and monocytes). The importance of $\mathrm{HOCl}$ formation is perhaps best illustrated by the fact that $5 \%$ of total neutrophil protein is myeloperoxidase. (47) Although not fully understood, $\mathrm{HOCl}$ has interesting biological properties that are distinct from peroxide.(48) $\mathrm{HOCl}$ reacts rapidly with glutathione (GSH) $\left(10^{7} \mathrm{M}^{-1} \mathrm{~s}^{-1}\right)(47)$ and NAC $\left(10^{8} \mathrm{M}^{-1} \mathrm{~s}^{-1}\right)$.(49) Intriguingly, the rate of reaction of $\mathrm{HOCl}$ with methionine is similar to the rate of $\mathrm{HOCl}$ oxidation of thiols $\left(10^{7}-10^{8} \mathrm{M}^{-1} \mathrm{~s}^{-1}\right)$.(49)This observation likely reflects the fact that $\mathrm{HOCl}$ is appreciably deprotonated at neutral $\mathrm{pH}\left(\mathrm{p} K_{\mathrm{a}} \mathrm{HOCl}: 7.5 ;(50)\right.$ $\mathrm{p} K_{\mathrm{a}} \mathrm{H}_{2} \mathrm{O}_{2}: 11.7$ in water), meaning that thiolate formation does not accelerate the reaction as efficiently as for the analogous oxidation by $\mathrm{H}_{2} \mathrm{O}_{2}$ (that is greatly accelerated by thiolate formation).(51)

$\mathrm{HOCl}$ affects cellular glutathione pools differently to treatment with standard oxidants that form glutathione disulfide. The product of human vascular epithelial cells (HUVEC) treated 
with $\mathrm{HOCl}$ is glutathione sulfonamide (GSA), a molecule that is rapidly excreted from cells. (52) (Figure S2) One explanation for this discrepancy is that a sulfenyl chloride is the initial product of the reaction between $\mathrm{RSH}$ and $\mathrm{HOCl}$. This intermediate may support an alternative reaction trajectory to the pathway that occurs when the sulfenic acid is formed. GSA formation may also reflect the fact that ${ }^{-} \mathrm{OCl}$ can behave as a nucleophilic oxidant,(53) meaning it has a higher propensity to oxidize electron-deficient sulfur than peroxide (whose rate of oxidation of sulfur decreases as sulfur oxidation state increases).

$\mathrm{HOCl}$ can react with amines to form chloramines. This process is around 100-fold slower than with thiols. Chloramines can also oxidize sulfur-containing amino acids, although the rates are relatively slow $\left(\mathrm{GSH}=115 \mathrm{M}^{-1} \mathrm{~s}^{-1}\right.$; methionine $\left.39 \mathrm{M}^{-1} \mathrm{~s}^{-1}\right)$. (51) In addition to amine chlorination, $\mathrm{HOCl}$ is also believed to chlorinate tyrosine,(54) likely through chloramine intermediates. The latter transfer chlorine in a fold-specific manner.(55) Chlorinated tyrosine modification is believed to be indicative of myeloperoxidase activity. (56) Although approximately 7-fold less potent than extracellular $\mathrm{H}_{2} \mathrm{O}_{2}$ treatment, extracellular $\mathrm{HOCl}$ treatment can also initiate tyrosine phosphorylation and TNF-a production, meaning that $\mathrm{HOCl}$ can elicit biologically relevant signaling.(57)

\subsubsection{ROS generation in mammalian cells: location, location, location?-An} important feature of signaling networks is the high level of protein target specificity demonstrated by cellular messengers. This is most readily exemplified by canonical, enzyme-assisted signaling processes, wherein a relatively unreactive precursor is activated and shepherded to a target protein by (an) enzymes(s). Many of these pathways-such as ubiquitination, SUMOylation, acetylation, methylation, phosphorylation and farnesylationuse inert precursors (Ub/SUMO/acetylCoA/S-adenosyl methionine or folate/ATP/ farnesylCoA) that are widely [although not necessarily uniformly $(113,114)$ ] distributed within the cell, with mediator enzyme's localization/expression/activity controlling signaling dynamics. Unlike these canonical systems, ROS (and RES) are highly reactive, short-lived and can modify many targets, largely in the absence of enzyme assistance.(17,115-117) It is thus likely that ROS/RES signals will not distribute evenly through the cell, meaning that location of generation will be a significant factor determining which signaling pathways are triggered. It must further be stressed that reactive molecules can have different fates due to context/location-specific regulation even within specific organelles.(118) We thus first focus on synthesis pathways (Figure 1).

\subsubsection{Superoxide generation at biological membranes by NADPH oxidase: ROS} generation by NOX is highly regulated, making NOX enzymes uniquely suited for redox signaling.(119) NOX proteins transfer electrons across biological membranes (Figure 2A) to reduce $\mathrm{O}_{2}$ to either $\mathrm{O}_{2}{ }^{\bullet-}$, or as discussed above $\mathrm{H}_{2} \mathrm{O}_{2}$ in the case of NOX4. ROS generation by NOX is initiated by NADPH as the 2-electron donor, and its subsequent transfer through FAD and heme cofactors to $\mathrm{O}_{2} \cdot(119)$

NOX were first identified in phagocytic cells such as macrophages and neutrophils. These cells generate a 'respiratory burst', a sudden generation of ROS that is used to combat invading microorganisms. The respiratory burst was attributed to the presence of gp91 phox (also known as NOX2), the transmembrane catalytic subunit of phagocytic NADPH oxidase, 
located on the cell membrane.(120) The discovery of other components of the phagocytic NOX enzyme system including the transmembrane protein $\mathrm{p} 22^{\text {phox }}$, and cytosolic subunits $\mathrm{p} 40^{\text {phox }}, \mathrm{p} 47^{\mathrm{phox}}$ and $\mathrm{p} 67^{\mathrm{phox}}$ followed.(119) The amino terminal region of NOX2 forms six transmembrane helices.(121) The region also contains five histidine residues, four of which are required for coordinating two hemes. The cytoplasmic C-terminus of the enzyme houses the binding sites for NADPH and FAD. Activation of NOX by exposure to microorganisms and inflammatory stimuli requires the recruitment of various cytosolic regulatory elements p40 $0^{\text {phox }}, \mathrm{p} 47^{\text {phox }}$, p67phox , and Rac1 to the membrane where they form a complex with cytochrome $b_{558}$ (gp91 ${ }^{\text {phox }}$ and $\mathrm{p} 22^{\text {phox }}$ ) (Figure $2 \mathrm{~A}$ ). The activation domain of $\mathrm{p} 67^{\text {phox }}$ initiates the rate-limiting electron transfer step from NADPH to FAD. The electrons are subsequently passed through two heme cofactors in the transmembrane domain to $\mathrm{O}_{2}$ that acts as the terminal electron acceptor, generating two $\mathrm{O}_{2}{ }^{-}$per NADPH used.

NOX enzymes are present in other cell types including fibroblasts, and vascular smooth muscle cells.(121) NOX1-a homolog of phagocytic gp91 phox — was isolated from colon epithelial cells.(122) Subsequently, NOX3(123) and NOX4(124) were identified in fetal tissues as well as in kidney, placenta, and glioblastoma cells. NOX5-expressed in the spleen, testis, and the lymph nodes-has an additional cytosolic calmodulin-like domain containing four calcium binding sites.(125) Unsurprisingly, NOX5 is stimulated by the calcium ionophore, ionomycin. In addition, a number of external signals such as growth factors, cytokines, and mechanical and elastic stress of epithelial cells trigger $\mathrm{O}_{2}$ - generation by NOX enzymes. Two other NOX homologs-DUOX1 and DUOX2(126) contain an additional extracellular peroxidase-homology domain at the N-terminus.(121) This functional coupling of ROS generation with its breakdown by the peroxidase-homology domain on a single gene underscores the importance of regulated ROS homeostasis.

In addition to plasma membrane-bound isoforms, NOX isoforms localize to subcellular organelles such as the endoplasmic reticulum (ER),(127) the nucleus, $(128,129)$ and the mitochondria.(130) NOX isoforms adopt different localizations in different cell types, indicating a complex context-dependent network controlling redox signal origin in cells. (131) NOX4, for example, was identified in the nucleus of HUVECs.(128) Nuclear fraction of HUVECs showed NADPH-dependent $\mathrm{O}_{2}{ }^{-}$-generation upon stimulation with phorbol ester, a positive regulator of protein kinase $\mathrm{C}$ that stimulates NOX activity. Nuclear NOX4 stimulation facilitates transactivation of the antioxidant response element (ARE) and siRNA knockdown of NOX4 abrogates this response. Certain isoforms of NOX, such as NOX5, also show variable localization. In the case of $\mathrm{NOX} 5, \mathrm{PI}(4,5) \mathrm{P}_{2}$ stimulates recruitment of the enzyme to the cell membrane via an $\mathrm{N}$-terminal polybasic region, leading to upregulation in extracellular ROS generation upon ionomycin stimulation of cells co-expressing NOX5 and PI4P kinase a.(132)

2.1.2.2 Mitochondrial generation of $\mathrm{O}_{2}{ }^{-}-$and $\mathrm{H}_{2} \mathrm{O}_{2}$ : Mitochondria are an important source of $\mathrm{O}_{2}{ }^{-}$and $\mathrm{H}_{2} \mathrm{O}_{2}$ (generated from $\mathrm{O}_{2}{ }^{-{ }^{-}}$-dismutation) in eukaryotic cells.(133) Mitochondrial $\mathrm{O}_{2}{ }^{-}$-production is estimated to be up to $2 \%$ of total $\mathrm{O}_{2}$ consumed by the isolated organelle.(134) In vivo $\mathrm{O}_{2}{ }^{-}$-flux under physiological conditions, however, is estimated to be significantly less $\left(<0.2 \%\right.$ of total $\mathrm{O}_{2}$ consumed by the organelle).(135) The steady-state $\mathrm{O}_{2}{ }^{-}$-concentration in mitochondrial matrix is estimated to be $10-200 \mathrm{pM}$ (Table 
1). $(24,134)$ These low levels are maintained by the action of manganese superoxide dismutase (Mn-SOD) —an important $\mathrm{O}_{2}{ }^{\circ}$ scavenger in mitochondria—present in micromolar concentrations in the matrix (Table 2).(101,133) Mitochondria-specific peroxiredoxins (Prx3 and Prx5) and glutathione peroxidase 1 (Gpx1) can further reduce the $\mathrm{H}_{2} \mathrm{O}_{2}$ generated to keep ROS levels in check. $(136,137)$ The significance of $\mathrm{O}_{2}{ }^{-}$-generation and clearance is perhaps best demonstrated by the fact that mice lacking mitochondrial MnSOD display postnatal lethality.(138) Heterozygotes are apparently normal, although mild elevation of some oxidative markers has been reported.(139) On the other hand, mice lacking extracellular SOD are normal.(140)

The production of $\mathrm{O}_{2}{ }^{-}$-primarily occurs at two points in the ETC, Complex I (NADH dehydrogenase) and Complex III (ubiquinone-cytochrome c reductase). Complex II also generates $\mathrm{O}_{2}{ }^{-}$under certain conditions.(133,141,142) (Figure 2B) Complex I and Complex II funnel electrons from reduced metabolites such as NADH and $\mathrm{FADH}_{2}$ to the coenzyme Q $(\mathrm{CoQ})$ reduction site through a chain of iron-sulfur $(\mathrm{FeS})$ centers. The electrons are then further transported to Cytochrome C (Cyt C) via Complex III that consists of 11 polypeptides, three hemes, and one FeS. From Cyt $\mathrm{C}$, ultimately, the electrons are transferred to Complex IV where they reduce ${ }^{3} \mathrm{O}_{2}$ to water. Flux through the ETC is also coupled with an ATP synthase that harnesses the energy of a transmembrane proton gradient to generate ATP.(143)

$\mathrm{O}_{2}{ }^{-}$-is produced in the mitochondria when electrons passing through ETC leak into the intermembrane region (Complex III) or the matrix of the mitochondria (Complex I and Complex III) (Figure 2B).(135) Experiments using isolated mitochondria show that Complex I generates superoxide in the mitochondrial matrix mainly by two mechanisms. $(147,148)$ In the first mechanism, $\mathrm{O}_{2}{ }^{-}-$is generated by the reaction of $\mathrm{O}_{2}$ with reduced FMN. $(148,149)$ Since the fraction of reduced FMN is determined by the NADH/NAD ${ }^{+}$ ratio, high $\mathrm{NADH} / \mathrm{NAD}^{+}$favors $\mathrm{O}_{2}{ }^{-}$-generation. The second mechanism involves reverse electron transfer (RET).(133) RET occurs when CoQ is reduced. Under high proton motive force, an electron is shuttled back from reduced $\mathrm{CoQ}\left(\mathrm{CoQH}_{2}\right)$ to Complex I, resulting in $\mathrm{O}_{2}$ - -generation. $(150,151)$ Complex III generates $\mathrm{O}_{2}{ }^{-}$-by the reaction of $\mathrm{O}_{2}$ with ubisemiquinone bound to the complex.(152) The superoxide generated at Complex III is released both in the mitochondrial matrix and the inner mitochondrial membrane space. (153) In principle, $\mathrm{O}_{2}{ }^{-}$-formed at either site can have signaling roles. However, considering the short half-life of $\mathrm{O}_{2}{ }^{\circ-}$, generation at Complex III-requiring traversing the membrane just once for cytosolic entry-may be more likely to elicit ROS signaling.(154)

Other $\mathrm{O}_{2}{ }^{-}$-generation sites in mitochondria include a-ketoglutarate and pyruvate dehydrogenase complexes.(155) Both enzymes house a flavin cofactor, the reduced form of which can reduce ${ }^{3} \mathrm{O}_{2}$ to $\mathrm{O}_{2}{ }^{--}$. Similar to Complex I, this reaction is enhanced when the mitochondrial electron acceptor $\mathrm{NAD}^{+}$is in short supply.(151) Additionally, p66shc, a mitochondrial intermembrane protein, enhances $\mathrm{O}_{2}{ }^{-}$production in response to apoptotic signals such as UV-irradiation and growth-signal deprivation in mammalian cells.(156) An inactive dimer, p66shc, upon stimulation with pro-apoptotic signals, converts to an active tetramer, which interacts with complex III to channel electrons away from cytochrome C. The oxidation of cytochrome $\mathrm{C}$ is coupled with the reduction of ${ }^{3} \mathrm{O}_{2}$ to generate $\mathrm{O}_{2}{ }^{-} \cdot \mathrm{H}_{2} \mathrm{O}_{2}$ 
resulting from $\mathrm{O}_{2}{ }^{--}$dismutation induces swelling of the mitochondria and opening of the permeability transition pore, key events in apoptosis.(156)

2.1.2.3 ER-mediated $\mathrm{O}_{2}{ }^{-}$and $\mathrm{H}_{2} \mathrm{O}_{2}$ generation: The ER lumen is one of the most oxidizing environments in the cell. The ER redox potential is estimated to be $-189 \mathrm{mV}$, considerably higher than mitochondria $(-280 \mathrm{mV})$ and cytosol $(-220 \mathrm{mV}) .(157)$ The oxidative environment of ER facilitates oxidative folding of proteins and disulfide bond formation. $(158,159)$ Protein disulfide bond formation is a redox process and in eukaryotic cells is catalyzed by several oxidoreductases including protein disulfide isomerase (PDI), (160) ERp61,(161) and ERp72.(161,162) Around 20 distinct oxidoreductases are found in the ER, each with unique redox potential and substrate specificity.(163) In PDI-catalyzed protein disulfide bond formation, cysteine residues in PDI serve as the acceptors of 2 electrons from cysteine residues of the substrate protein.(164) The reduced PDI is regenerated via multiple pathways.(159,165) The flavoenzyme ER oxidoreductase (Ero1) acts an important re-oxidizing protein for PDI.(166) This reoxidation step must be coupled to concomitant reduction of another substrate. In the ER lumen, ${ }^{3} \mathrm{O}_{2}$ serves as the ultimate oxidant, which is reduced to $\mathrm{H}_{2} \mathrm{O}_{2}$ in the process. $(167,168) \sim 25 \%$ of all $\mathrm{H}_{2} \mathrm{O}_{2}$ produced in yeast cells is generated by the action of Erolp-mediated disulfide bond formation. $(158,168,169)$ Additionally, ER-resident Prx 4 and Gpx7/8 also reoxidize PDI, consuming $\mathrm{H}_{2} \mathrm{O}_{2}$ in the process. Furthermore, non-enzymatic routes of PDI re-oxidation including those mediated by GSSG, dehydroascorbate, and $\mathrm{H}_{2} \mathrm{O}_{2}$ have also been described.(165) Finally, members of the quiescin-sulfhydryl oxidase (QSOX) family of flavoenzymes can directly catalyze the oxidation of peptide/protein thiols into disulfides with the accompanying reduction of molecular oxygen to $\mathrm{H}_{2} \mathrm{O}_{2}$. (170)

Microsomal monooxygenases (MMOs) are another important source of $\mathrm{O}_{2}{ }^{\cdot-}$ and $\mathrm{H}_{2} \mathrm{O}_{2}$ in the ER. MMOs are a multi-enzyme system comprising of a terminal cytochrome $\mathrm{P} 450$ (CYP450), which receives electrons from a FAD/FMN-containing NADPH cytochrome P450 reductase (CPR).(171,172) The primary function of MMO is the oxidation of external xenobiotics, small-molecule inhibitors/drugs, and endogenous metabolites. During monooxygenase reaction, a two-electron transfer is initiated by CPR using NADPH.(172) The electrons are relayed through CPR via FAD/FMN. One electron is accepted by the ferric iron (III) of the heme cofactor in CYP450 resulting in the ferrous (II) intermediate(172) (Figure 2C). This intermediate then binds to oxygen generating an oxycomplex, followed by a peroxy intermediate eventually forming a Compound I [ $\mathrm{Fe}(\mathrm{IV})=\mathrm{O}$; iron(IV)oxo] species that catalyzes the oxidation of substrates. In eukaryotes, a significant fraction of the activated oxygen in oxy- and peroxy-complex is released via multiple mechanisms without being used for substrate oxidation. One such mechanism involves the decay of the oxycomplex to generate $\mathrm{O}_{2}{ }^{-}$. Another mechanism involves protonation and subsequent uncoupling of the peroxy intermediate to generate $\mathrm{H}_{2} \mathrm{O}_{2}$ (Figure 2C).(149,172) MMOs have relatively poor second-order oxidation kinetics. For instance, Flavin-containing monooxygenase 3 has $k_{\mathrm{cat}} / K_{\mathrm{m}}=6700 \mathrm{M}^{-1} \mathrm{~s}^{-1}$ for the Aurora kinase inhibitor, Tozasertib, (173) and $3200 \mathrm{M}^{-1} \mathrm{~s}^{-1}$ for Danusertib,(174) another Aurora kinase inhibitor. There is also a low coupling efficiency (substrate turnover per molecule of NADPH used) of $\sim 60 \%$.(172) For many substrates, the coupling efficiencies are $<10 \%$ suggesting that a large fraction of 
activated oxygen leaks out as ROS.(175) Cytochrome b5, another membrane hemoprotein, was found to protect against ROS generation by increasing MMO activity and coupling efficiency.(175)

ER-localized NOX enzymes are yet another source of ROS. NOX4 generates $\mathrm{O}_{2}{ }^{-}$and $\mathrm{H}_{2} \mathrm{O}_{2}$ in the ER lumen thus maintaining the oxidative environment. $(25,26,168)$ Unfolded protein response (UPR) activation in peripheral vasculature cells is a means to activate NOX4 that results in increased ER ROS generation.(176)

2.1.2.4 Redox regulation of GSH in the ER versus the mitochondrion: Like other cellular compartments such as the mitochondria, redox status of the ER is also kept in check by various reducing agents.(177) GSH transported from the cytosol acts as the major reducing agent both in the ER and the mitochondria. Selective transport of GSH across the ER, a process that in yeast occurs through the ER channel Sec 61,(178) has been demonstrated in rat liver microsomal vesicles.(179) GSH transport into the sarcoplasmic reticulum of skeletal muscles occurs via ryanodine receptor calcium channel type1.(180) Additionally, the ER membrane is also permeable to small molecules,(181) perhaps allowing passive diffusion of GSH across the gradient from cytosol to the ER.(178) Despite the report of both active and passive diffusion into the ER, GSH concentration in the ER is kept low by multiple mechanisms. GSH is actively consumed in the ER to reduce incorrectly oxidized proteins. (182) Unsurprisingly, a significant amount of GSH forms mixed disulfides with ER proteins. (183) Additionally, $\mathrm{H}_{2} \mathrm{O}_{2}$ generated during oxidative folding of proteins mediated by PDI/ Ero1 oxidizes GSH.(167) The high concentration of $\mathrm{H}_{2} \mathrm{O}_{2}$ generated in the ER may help offset the low reactivity of GSH with $\mathrm{H}_{2} \mathrm{O}_{2}$ (second order rate constant of $\sim 1 \mathrm{M}^{-1} \mathrm{~s}^{-1}$ at $\mathrm{pH}$ 7.5 and at $\left.37^{\circ} \mathrm{C}\right) .(66)$ Additionally, ER-localized glutathione peroxidases such as Gpx7 and Gpx8 may accelerate the reaction(184) although recent reports suggest that neither have significant Gpx activity, instead preferring oxidized PDI as a substrate.(185)

GSH cannot cross the inner mitochondrial membrane due to its anionic nature at physiological $\mathrm{pH}$ and the negative membrane potential of the mitochondrial matrix.(186) Despite the physical barrier to GSH entry, mitochondria maintain a more reductive environment compared to the ER. For example, the GSH:GSSG ratio in ER is maintained around 3:1-1:1.(187) The ratio in cytosol and mitochonrdia ranges from 30:1-100:1.(187) The GSH concentration in mitochondria is around 10-14 mM.(188) Dicarboxylate and the a-ketoglutarate carrier proteins were suggested to mediate this transport; however, recent studies have disputed these findings(189) suggesting the existence of a yet unknown transport mechanism. Additionally, mitochondrial GSSG is reduced in the matrix by glutathione reductases using NADPH as the reductant thus maintaining high levels of mitochondrial GSH.(186)

2.1.2.5 Peroxisomal generation of ROS: Peroxisomes are multi-purpose organelles important for processes such as oxidation of fatty acids, catabolism of purines, and biosynthesis of glycerol-lipids and bile acids.(190) The central role of peroxisomes in the maintenance of redox homeostasis is exemplified by the fact that peroxisomes consume as much as $20 \%$ of cellular oxygen taken up by a cell and as they release $20-60 \%$ of the peroxide they generate(191) (likely through a specific porin-like channel).(192) Ultimately, 
this organelle generates approximately $35 \%$ of cellular peroxide. $(190,191)$ Peroxisome and mitochondria share the ability for cooperative $\beta$-oxidation of long chain fatty acids. These two organelles coordinate the generation and/or scavenging of ROS based on cellular needs. Peroxisomes house a battery of antioxidant enzymes including catalase, SODs, etc. to maintain redox balance.(193) Peroxisomes also contain various oxidases including D-amino acid oxidase, D-aspartate oxidase, urate oxidase, palmityl-CoA oxidase, etc., for the oxidation of various metabolites. Many of these oxidases are FAD/FMN-dependent oxidases that generate $\mathrm{O}_{2}{ }^{-}{ }^{-}$and $\mathrm{H}_{2} \mathrm{O}_{2}$ as byproducts. $(118,149)$ Additionally, xanthine oxidase and nitric oxide synthase generate $\mathrm{O}_{2}{ }^{\cdot-}$ and nitric oxide ('NO), respectively.(193)

Unsurprisingly, peroxisomes likely play important roles in redox signaling, although the ins and outs of these processes still need to be clarified.(194) Indeed, overexpression of fatty acyl CoA oxidase (a protein that generates peroxide in the peroxisome) in the presence of an $\mathrm{H}_{2} \mathrm{O}_{2}$-generating substrate stimulates NF- $\mathrm{kB}$ (a redox-controlled transcription factor) signaling.(195) Conversely, compromised activity of catalase, an antioxidant enzyme localized in peroxisomes, is associated with a number of human pathologies including hypertension, type 2-diabetes, and neurodegenerative diseases.(193)

2.1.2.6 Other sources of ROS: In addition to endogenous sources, a variety of exogenous stimuli also generate ROS in cells. Environmental sources such as UV light,(196) ionizing radiation,(59) and xenobiotics(172) can generate a variety of ROS. 1,2 and 1,4-benzenediols (such as catechol, resorcinol, and hydroquinone) and endogenously-generated catecholamines (dopamine, adrenaline, etc.) that are metabolized to quinone and semiquinone derivatives in cells also enable ROS generation in presence of trace metals. (197-199) Quinones and similar species can also behave as RES (see section 2.2.1.4). Whether the ROS modulating properties of RES are linked to disease and signaling is an ongoing area of debate.(200)

2.1.2.7 Interconversion of ROS: $\mathrm{O}_{2}{ }^{-\boldsymbol{}}$ generated by NOX enzymes can undergo chemical reactions to generate secondary oxidative species (Figure 3). $\mathrm{O}_{2}{ }^{\cdot-}$ can rapidly dismutate, either spontaneously or enzymatically, to $\mathrm{H}_{2} \mathrm{O}_{2}$ and $\mathrm{O}_{2}$. The rate constant for the spontaneous dismutation of $\mathrm{O}_{2}{ }^{-}-$is $\sim 10^{5} \mathrm{M}^{-1} \mathrm{~s}^{-1}$ at neutral $\mathrm{pH}$ in aqueous solution.(201) However, this reaction is bimolecular, and accordingly, the half-life of $\mathrm{O}_{2}{ }^{-1}$ depends on the $\mathrm{O}_{2}{ }^{\cdot-}$ concentration. SODs are highly abundant in eukaryotic cells (e.g. [Cu/Zn-SOD] in the cytosol of rat hepatocytes $\sim 1.36 \mathrm{mg} / \mathrm{mL}, 24 \mu \mathrm{M}$ ), and thus help maintain a low steady-state concentration of $\mathrm{O}_{2}{ }^{-}-(19,202)$ SODs increase the $\mathrm{O}_{2}{ }^{-}-$dismutation rate by four orders of magnitude to the diffusion limit $\left(\sim 10^{9} \mathrm{M}^{-1} \mathrm{~s}^{-1}\right)$. Unlike spontaneous dismutation, the reaction between $\mathrm{O}_{2}{ }^{-}$and SOD is of first order, rendering half-life independent of $\mathrm{O}_{2}{ }^{-}$concentration.(202)

$\mathrm{O}_{2}{ }^{\bullet-}$, although not very reactive with amino acids,(204) reacts with ${ }^{\bullet} \mathrm{NO}$ at diffusioncontrolled rates (approximately $10^{10} \mathrm{M}^{-1} \mathrm{~s}^{-1}$ ) to generate $\mathrm{ONOO}^{-}$.(205) Indeed in some cases this reaction may proceed faster than SOD-catalyzed dismutation.(72) $\mathrm{ONOO}^{-}$ intrinsically decays to ${ }^{\circ} \mathrm{OH}$ and ${ }^{\circ} \mathrm{NO}_{2}\left(k\right.$ approximately $\left.1 \mathrm{~s}^{-1}\right)$ around neutral $\mathrm{pH} .(73,206)$ $\mathrm{ONOO}^{-}$can also modify proteins, oxidize thiols, and react with fatty acids to generate RES (see Section 2.2.1.3).(207) These modification processes occur with rate constants on the 
order of $10^{2}$ to $10^{6} \mathrm{M}^{-1} \mathrm{~s}^{-1}$ and are believed to dominate over the intrinsic decomposition reaction (at least at neutral $\mathrm{pH}$, although this is not necessarily true in acidic vesicles).(208210). The most favored reaction for $\mathrm{ONOO}^{-}$is the one-electron reaction with $\mathrm{CO}_{2}$ to generate carbonate radical $\left[\left(\mathrm{CO}_{3}{ }^{-}, \mathrm{E}^{0}=1.8 \mathrm{~V}\right.\right.$; diffusion distance $\left.152 \mathrm{~nm}\right)$ and ${ }^{\circ} \mathrm{NO}_{2}\left(\mathrm{E}^{0}=\right.$ $1.0 \mathrm{~V}$; diffusion distance $188 \mathrm{~nm}) ;(116,211,212)$ (a process that occurs through a transient species that forms with a rate constant of $\left.\left.3 \times 10^{4} \mathrm{M}^{-1} \mathrm{~s}^{-1}\right)\right] . \mathrm{CO}_{3}{ }^{-}$, although less oxidizing than ${ }^{\circ} \mathrm{OH}\left(\mathrm{E}^{0}=2.3 \mathrm{~V}\right),(211)$ reacts with various amino acids with high second-order rate constants $\left[10^{6}-10^{8} \mathrm{M}^{-1} \mathrm{~s}^{-1}\right.$,(213) Cf. $10^{9}-10^{10} \mathrm{M}^{-1} \mathrm{~s}^{-1}$ for $\left.{ }^{\bullet} \mathrm{OH}(214)\right] . \mathrm{CO}_{3}{ }^{-}$shows some kinetic selectivity for tryptophan oxidation, a process that produces proteins linked through tryptophan dimers.(215) This may be a means to identify carbonate radical chemistry in cells, although this idea has not been fully expanded,(216) and likely is not fully diagnostic as multiple oxidative processes can lead to this product.

• $\mathrm{OH}$ is also generated from $\mathrm{O}_{2}{ }^{\cdot-}$ and $\mathrm{H}_{2} \mathrm{O}_{2}$ in the presence of trace metals through the Fenton/Haber-Weiss reactions. $\mathrm{H}_{2} \mathrm{O}_{2}$ can oxidize $\mathrm{Fe}^{2+}$ to generate ${ }^{\circ} \mathrm{OH}$ and the hydroxide anion. $(217,218)$ The rate constant for this reaction carried out by the iron hexa-aquo complex is reported to be $\sim 100 \mathrm{M}^{-1} \mathrm{~s}^{-1}$ at room temperature. $(219,220)$ The reaction rate varies significantly depending on the nature of ligands bound to iron (and their relative concentrations), the presence of reducing agents(221) and $\mathrm{pH} .(220)$ The rate constants quoted for different liganded iron species range at least between $10^{4}$ and $10^{-4} \mathrm{M}^{-1} \mathrm{~s}^{-1}$.(221) For instance, ATP and pyrophosphate [hard, anionic donor ligands that stabilize the (hard) $\mathrm{Fe}(\mathrm{III})$ form] suppress the Fenton reaction,(222) although this relationship is likely concentration dependent. Given the uncertain nature of the cellular labile iron pool,(223) it is thus hard to predict the rate of Fenton reaction in cells and hence the contribution of Fenton chemistry to ROS generation is likely to be context dependent.

The diverse chemistry undertaken by iron highlights the complexity of the interplay of ROS. For instance, SOD catalyzes the formation of 0.5 equivalents of $\mathrm{H}_{2} \mathrm{O}_{2}$ from each $\mathrm{O}_{2}{ }^{\cdot-}$. Thus, one could propose that SOD promotes peroxide formation in cells. Evidence has been provided that agrees with this hypothesis.(224) However, this argument ignores that $\mathrm{O}_{2}{ }^{-}$ has pleotropic effects on cells; it can disrupt many $4 \mathrm{Fe}-4 \mathrm{~S}$ centers, affecting the concentration of reduced iron in cells. The free iron generated can ultimately generate peroxide catalytically, and may actually upregulate peroxide generation over that in the presence of SOD. Indeed, overexpression of SOD leads to lower peroxide levels in Chinese hamster cells than parental cells.(225)

$\mathrm{HOCl}$, generated by myeloperoxidase, can react with peroxide anion to yield ${ }^{1} \mathrm{O}_{2 .}$ (199) Using diphenyl anthracene, a ${ }^{1} \mathrm{O}_{2}$ trap, it was estimated that $\sim 20 \%$ of the $\mathrm{O}_{2}$ consumed by neutrophils is used to generate ${ }^{1} \mathrm{O}_{2},(226)$ although subsequent studies have downplayed the importance of this process. $(83,227,228)$

Ultimately, the rapid interconversions of ROS, which often occur in a context-dependent manner, make it difficult to parse the most biologically-relevant ROS in cells. $\mathrm{H}_{2} \mathrm{O}_{2}$ because of its relatively long half-life and resulting diffusion distance (see section 3.1) compared to $\mathrm{O}_{2}{ }^{\cdot-},{ }^{\bullet} \mathrm{OH}$ and ${ }^{1} \mathrm{O}_{2}$-is thought to be the most important ROS in signaling. However, this notion could perhaps be a consequence of lack of tools to measure short-lived 
and more reactive ROS such as ${ }^{\bullet} \mathrm{OH}$ and ${ }^{1} \mathrm{O}_{2}$. Indeed, the high reactivity of species like ${ }^{\bullet} \mathrm{OH}$ and $\mathrm{ONOO}^{-}$can be harnessed to engender specificity in target modification. A two-step hypothesis has been proposed for the signaling roles of highly reactive radicals.(229) The first step involves a diffusion-controlled reaction of proteins with reactive radicals such as - OH to generate a stable protein radical. In the second step, the stable protein radical can react with ROS of relatively longer half-life such as $\mathrm{O}_{2}{ }^{--}$. This mechanism is exemplified in the oxidation/nitration of tyrosine residues where a more stable phenoxy-radical is generated by the reaction of tyrosine with ${ }^{\circ} \mathrm{OH}$. This stable radical then reacts with other less-reactive radicals including $\mathrm{O}_{2}{ }^{-}{ }^{-}$and ${ }^{\circ} \mathrm{NO}$, yielding oxidized/nitrated tyrosine.(229)

2.1.3 Inter-organelle and inter-cellular redox information exchange-Organelles frequently crosstalk. As was discussed in 2.1.2.4, mitochondria and peroxisomes share numerous common pathways and can crosstalk.(230) Additionally, peroxisomal tethering to the ER may enable transport of lipids from the ER to peroxisome and plasminogen and cholesterol precursors from peroxisome to the ER.(231) The de novo synthesis of peroxisomes was recently shown to require fusion of pre-peroxisomes derived from both ER and mitochondria, thus establishing a functional coupling between these organelles.(232) Accumulating evidence demonstrates that deregulation in lipid metabolism and ROS generation in peroxisomes perturbs mitochondrial redox balance.(233) For example, inhibition of peroxisomal catalase activity in human fibroblast Hs27 cells treated with aminotriazole (an irreversible inhibitor of catalase), upregulates mROS generation and inhibits mitochondrial aconitase [a protein known to be highly ROS sensitive(234)].(235) Additionally, knockdown of ABCD1 - a peroxisomal very long chain fatty acid transporter - causes oxidative damage to mitochondrial proteins and plays a causative role in X-linked adrenoleukodystrophy, a metabolic disorder of the spinal cord. Spinal cords of $a b c d 1^{-/-}$ mice feature increased ROS generation in the mitochondria upon treatment with very long chain fatty acids resulting in oxidation of mitochondrial proteins, including proteins in the mitochondrial ETC and those involved in the TCA cycle.(236) The exact mechanisms of how treatment with very long chain fatty acids generate mitochondrial ROS in cells deficient for abcd1 is still unclear.

ER and mitochondrial ROS generation are also interconnected. Menadione-induced ER stress resulting in $\mathrm{Ca}^{2+}$ release in the cytosol of pancreatic acinar cells increases mitochondrial ROS generation leading to apoptosis by induction of the mitochondrial permeability transition pore complex.(237) Additionally, mitochondrial dysfunction elicits increased ROS generation in the ER. During glucose starvation in yeast (which activates oxidative phosphorylation that occurs in the mitochondria), inactivation or loss of cytochrome-C oxidase (CCO) of the mitochondrial ETC results in upregulation of ROS generation from the surface of the ER.(238) CCO-deficient mitochondria recruit and activate Ras upon their outer membrane by an unknown mechanism. Activated Ras suppresses ERassociated protein degradation (ERAD), stabilizing ER-localized NOX, Ynop1, enabling the generation of $\mathrm{O}_{2} \cdot{ }^{\cdot-} \cdot(238,239)$

There are also examples in which ROS mediates intercellular communication. This is most notably demonstrated in the case of $\mathrm{H}_{2} \mathrm{O}_{2}$. The low reactivity of $\mathrm{H}_{2} \mathrm{O}_{2}$ and its long half-life enable large diffusion-distances $(\sim 0.5 \mu \mathrm{m})$ (Table 1)(59) more-suited for intercellular 
communication. It is worth noting that plasma membrane-bound NOX-dependent $\mathrm{H}_{2} \mathrm{O}_{2}$ is generated in the extracellular matrix, which is then transported into the cell. There is evidence that some of the $\mathrm{H}_{2} \mathrm{O}_{2}$ generated extracellularly may act as intercellular redox signals.(240) ROS generated in the mitochondria and activated myofibroblasts can act as signaling molecules inducing apoptosis in bystander cells.(241) Additionally, DUOXgenerated $\mathrm{H}_{2} \mathrm{O}_{2}$ is indispensable for rapid recruitment of leukocytes and efficient wound healing in zebrafish.(242) Lyn, a Src family kinase, functions as a $\mathrm{H}_{2} \mathrm{O}_{2}$ sensor mediating neutrophil recruitment to wound site in zebrafish larvae. Specific mutation of C466 from seven total cysteines on the neutrophilic Lyn abolishes its redox sensing ability and consequent recruitment of neutrophils.(243)

\subsection{Reactive electrophilic species (RES)-mediated signal transduction}

RES are now acknowledged to be important cellular signals.(115) A wide range of electrophiles have been shown to modulate several critical signaling pathways including antioxidant response (AR), apoptosis, DNA damage response, and cell growth. Biological electrophiles come in a variety of shapes and sizes ranging from formaldehyde to 12-nitroarachidonic acid (12- $\left.\mathrm{NO}_{2}-\mathrm{AA}\right)$, and epoxides to steroidal $\alpha, \beta$-unsaturated carbonyls that are found in prostaglandins (Figure 4).(115) Given the broad range of functionality but overlapping chemical reactivity within RES, study of biological RES is a challenge.

Considerable effort has been made to understand the chemical principles underlying the reaction between electrophiles and nucleophiles. One of the more widely-used models is the hard/soft acid-base model (HSAB).(244,245) HSAB depicts two extremes of nucleophiles/ electrophiles. Hard nucleophiles/electrophiles are often charged and non-polarizable. Soft nucleophiles/electrophiles typically have a delocalized charge density and are polarizable. According to HSAB, hard electrophiles prefer to react with hard bases and soft electrophiles with soft bases. The basis for this observation is typically ascribed to domination of charge/ charge interactions for "hard" and domination of HOMO-LUMO overlap for "soft" interactions.(244) One consequence of these differences is that Hard interactions tend to proceed through an early transition state, whereas soft interactions tend to go through later transition states.(246) Thus, there is a significant difference in charge distribution and bond formation in the different adduction transition states. The most signaling-relevant electrophiles are typically 'soft' Michael acceptors that react with 'soft' nucleophiles, principally thiols. There is evidence that amine-based nucleophiles [from proteins or other small molecules $(247,248)]$ may be able to adduct to soft electrophiles, especially if exposure time is increased. Most adducts to RES are stable under mild conditions (neutral $\mathrm{pH}$, and ambient temperatures) regardless of the nature of the bond formed between the electrophile and the nucleophile. However, most adducts are detected either by SDS-PAGE gel, or through direct mass spectrometry (MS). There is good evidence to show that many amine adducts are more stable than adducts to thiols, possibly explaining why histidine/lysine adducts have been reported by many researchers.

2.2.1 Lipid peroxidation products and RES generation-Lipid-derived electrophiles (LDEs) are made endogenously via both enzymatic and non-enzymatic pathways.(115,249) Polyunsaturated fatty acids (PUFAs) are an important source of 
endogenous LDEs. Peroxidation of PUFAs, either through enzymatic(250) or non-enzymatic pathways,(115) results in the generation of a variety of electrophilic compounds capable of signaling roles.(251) a, $\beta$-unsaturated carbonyls are present in micromolar quantities in the resting state in cells(84) but can rise much higher during stress.(85) (Table 1) These electrophiles modulate numerous processes in human biology - from development $(252,253)$ and aging(254) to cellular homeostasis events such as apoptosis,(255) immune response, $(115,256)$ and heat shock response.(115) For instance, $15 \mathrm{~d}-\mathrm{PGJ}_{2}$ is a potent immunemodulator that downregulates NF- $\kappa$ B pathway.(256) HNE inhibits 12-O-

tetradecanoylphorbol-13-acetate (TPA)-induced Jurkat T-cell activation by regulating NF- $\mathrm{BB}$ signaling. (257) HNE also activates the Keap1-Nrf2 antioxidant signaling response. $(258,259)$

2.2.1.1 Enzymatic lipid peroxidation pathways: Three classes of enzymes, working in concert or alone generate RES.(250) These enzymes consist of LOXs, $(13,249,260)$ COXs, $(11,261,262)$ and CYP450.(263) LDEs are primarily generated from two substrates: $\omega-3$ and $\omega-6$ fatty acid. An important substrate in the $\omega-6$ fatty acid pathway is arachidonic acid (AA). COX-and LOX-mediated oxidation of AA generates numerous secondary metabolites [only a fraction of which are electrophilic $(264,265)]$ with diverse biological activities/roles including regulating inflammation, $(266)$ vasodilation, $(265,266)$ and platelet aggregation. $(265,266)$

COXs are non-heme-iron-containing proteins that catalyze AA oxidation. Among the two mammalian COX isoforms, COX-1 is expressed in all tissues whereas COX-2 is limited to several tissues including kidney and brain $(267,268)$ and only induced in other tissues during inflammation.(269) Both COX-1 and COX-2 generate prostaglandin $\mathrm{H}_{2}\left(\mathrm{PGH}_{2}\right)$ from AAoxidation (Figure 5).(265) $\mathrm{PGH}_{2}$ is then further oxidized to other prostaglandins mediated by CYP450 prostaglandin synthetases.(265) Several prostaglandins (15d-PGJ $2, \mathrm{PGA}_{2}$ and $\mathrm{PGJ}_{2}$ ) contain a, $\beta$-unsaturated carbonyls making them electrophilic. In the case of $15 \mathrm{~d}-$ $\mathrm{PGJ}_{2}, \mathrm{PGH}_{2}$ is first oxidized by the action of prostaglandin D (PGD) synthetase into an unstable intermediate $\left(\mathrm{PGD}_{2}\right)$, which readily dehydrates to yield $\mathrm{PGJ}_{2}$.(270) $\mathrm{PGJ}_{2}$ can undergo spontaneous isomerization to generate ${ }^{12}-\mathrm{PGJ}_{2}$, which dehydrates to yield $15 \mathrm{~d}$ $\mathrm{PGJ}_{2}$ (Figure 5). In addition to AA, COX-2 also uses $\omega-3$ PUFA as a substrate to generate electrophilic lipid signals. COX-2 in activated macrophages stimulates the production of electrophilic oxo-derivatives from the $\omega-3$ fatty acids docosahexenoic acid (DHA) and eicosapentenoic acid. The process involves COX-2-catalyzed hydroxylation followed by dehydrogenases-assisted oxidation.(249)

2.2.1.2 Non-enzymatic lipid peroxidation: Non-enzymatic generation of LDEs is mediated by reactive intermediates, many of which can be formed by the interaction of redox active metals with more stable oxidants, such as peroxide.(115,271-274) ROS such as ${ }^{\circ} \mathrm{OH}(69)$ and RNS like ${ }^{\circ} \mathrm{NO}_{2}(205)$ react rapidly with immediately-accessible biological molecules. One of the key sites of reactive species generation is at the plasma membrane and membranes of other organelles (see section 2.1.2.1). PUFA-rich cell membranes undergo rapid hydrogen abstraction by radical oxidants. PUFA oxidation is favored due to the formation of stabilized carbon radicals and because $\mathrm{O}_{2}$ can concentrate in biological membranes.(275) Lipid 
oxidation is typically triggered by the kinetically-favored abstraction of the activated bisallylic hydrogen(276) by a radical oxidant such as $\mathrm{O}_{2}{ }^{-}{ }^{-}$or ${ }^{\bullet} \mathrm{OH}$ (Figure 6).(277) The resulting doubly-allylic radical reacts with ${ }^{3} \mathrm{O}_{2}$ to generate a lipid peroxide radical which abstracts hydrogen from another PUFA, forming a lipid hydroperoxide and a new lipid radical in a propagation step.(278) Lipid hydroperoxides generated during the reaction may undergo Hock cleavage (a process of 1,2-carbon migration in a peroxide to form a carbonyl and an alcohol) to generate LDEs such as HNE and 4-hydroxyhexenal (HHE), from $\omega-6$ and $\omega$-3 PUFAs, respectively (Figure 6).(277,279) LDEs may also undergo further oxidation to form the corresponding epoxy adducts either chemically or enzymatically.(280) Little is known about their specific chemistry in vivo. However, it is likely that their metabolic stability is different from canonical LDEs.(281) Aside from Hock cleavage, the lipid peroxide generated by ROS can cyclize, react with an additional ${ }^{3} \mathrm{O}_{2}$, and further rearrange to form various prostaglandin-like signaling molecules called isoprostanes (Figure 6). AAderived $\mathrm{E}_{2}$-and $\mathrm{D}_{2}$-isoprostanes dehydrate to generate electrophilic $\mathrm{A}_{2}$ - and $\mathrm{J}_{2}$-isoprostanes.

2.2.1.3 Nitro-fatty acids ( $\mathrm{NO}_{2}$-FA) generation: Nitroalkenes are relatively abundant in organisms. There is $\sim 1 \mathrm{nM}$ of nitro-conjugated linoleic acid in human plasma,(91) and 10's of $\mathrm{nM}$ in heart tissue under stress.(94) Biosynthesis of nitroalkenes is complex. ${ }^{\circ} \mathrm{NO}$ is mainly enzymatically generated by nitric oxide synthetases.(282) Although ${ }^{\circ} \mathrm{NO}$ is unreactive towards proteins,(5) it rapidly reacts with $\mathrm{O}_{2}{ }^{-}{ }^{-}$to generate $\mathrm{ONOO}^{-}$(Figure 3). These peroxynitrites generate ${ }^{\circ} \mathrm{NO}_{2}$ through processes discussed in 2.2.1.6.(283,284) ${ }^{\circ} \mathrm{NO}_{2}$ can abstract bisallylic hydrogen from PUFAs at diffusion controlled limits to initiate lipid peroxidation. This process forms a mesomerically stabilized carbon-centered radical (Figure 7).(115) When ${ }^{3} \mathrm{O}_{2}$ levels are high, this radical can be trapped by ${ }^{3} \mathrm{O}_{2}$ to form a peroxide. In low ${ }^{3} \mathrm{O}_{2}$ conditions, ${ }^{\circ} \mathrm{NO}_{2}$ reacts with PUFAs to generate $\mathrm{NO}_{2}$-FA.(285) The reaction can occur through direct addition to an olefin by ${ }^{\circ} \mathrm{NO}_{2}$ or with abstraction of a hydrogen radical from the doubly allylic methylene group within the PUFA by ${ }^{\circ} \mathrm{NO}_{2}$ radical. The latter proceeds through a similar doubly allylic radical. Under low ${ }^{3} \mathrm{O}_{2}$, another ${ }^{\circ} \mathrm{NO}_{2}$ reacts with the carbon-centered radical generating a short-lived intermediate nitroso ester that either hydrolyses or loses $\mathrm{NO}_{2} \mathrm{H}$ via $\mathrm{E} 1_{\mathrm{CB}}$ to form an $\alpha, \beta$-unsaturated nitro-FA. $\mathrm{NO}_{2}$-FA are important for cellular maintenance and act as ligands to receptors such as PPAR $\gamma$.(286) $\mathrm{NO}_{2}$-FA also inhibit tumor necrosis factor a (TNFa)-induced macrophage activation by regulating NF- $\mathrm{kB}$ signaling.(287) These molecules also activate the Keap1-Nrf2 antioxidant signaling response. $(258,259)$ Exogenous addition of nitro-oleic acid to mice was protected against myocardial ischemia reperfusion injury.(94) This was in part mediated through the downregulation of NF- $\mathrm{kB}$ pathway by inactivating the p65 subunit.(94)

In addition to nitrating lipids, $\mathrm{ONOO}^{-}$can also generate other electrophilic species, such as nitrated nucleotides. 8-nitro-cGMP is formed by the addition of $\mathrm{ONOO}^{-}$to cGMP.(288) 8nitro-cGMP can guanylate protein thiols.(289) For example, $S$-guanylation of Keap1 results in loss of its ability to bind Nrf2 and subsequently upregulates of a battery of AR genes. $(289,290) S$-guanylation of proteins also releases nitrite $\left(\mathrm{NO}_{2}^{-}\right)$, which can act as signaling molecule. $(291,292)$ 
2.2.1.4 Other metabolic processes that produce RES: Other RES can be formed by metabolic processes in cells, or are part of critical metabolic pathways. Fumarate is part of the citric acid cycle and is also a postulated oncometabolite(293) that can deplete glutathione and NADPH in cells.(294) Fumarate can also adduct to protein thiols(295) and amines.(295) Mutations in fumarate hydratase, a protein that is immediately downstream of fumarate in the citric acid cycle, can cause hereditary leiomyomatosis and renal cell cancer.(296) Protein succinylation (products of protein lysine adduction to fumaric acid) is elevated in cells from these patients. Fumarate is also believed to activate Keap1-Nrf2 pathway.(297) Another reactive electrophile derived from the citric acid cycle that has signaling roles(298) is itaconate, an enone synthesized in activated macrophages.(299,300) Methylglyoxal, a reactive dicarbonyl compound, can be formed from sugar oxidation. Interestingly, this compound builds ups in plant chloroplasts during photosynthesis.(301) In mammals, this species is formed both enzymatically and non-enzymatically through spontaneous hydrolysis of glyceraldehyde-3-phosphate or dihydroxyacetone phosphate (intermediates of glycolysis). (302-304).

Acrolein is also generated endogenously by the myeloperoxidase-mediated degradation of threonine(305) as well as various other metabolic processes.(306) While small concentrations may be beneficial,(306) acrolein can covalently conjugate with proteins and DNA resulting in electrophilic stress and cell death at higher concentrations.(307) Quinones can also be formed through normal metabolic processes. Two regioisomers of quinones can be made: 1,4-quinones ( $p$-quinones, e.g., ubiquinone) are typically more stable than their 1,2 -quinone regioisomers ( $o$-quinones), although this difference is functional group dependent. Tyrosinase is an enzyme that catalyzes the two-step oxidation of tyrosine to the corresponding $o$-quinone, a process that is important in the production of melanin. Tyrosinase generates $o$-quinones from L-DOPA and dopamine both of which can deplete glutathione and inhibit reverse transcriptase.(308) The action of tyrosinase on skinwhitening agents may also be responsible for leukoderma (vitiligo). (309) Critically, some tyrosinases can oxidize protein tyrosines leading to protein fragmentation, cross linking, or functionalization of tyrosine, depending on conditions.(310) Phenols, such as tyrosine, require enzymatic oxidation to form the $o$-quinone. However, 1,2-benzene diols are inherently prone to oxidation or can be oxidized enzymatically (by tyrosinase, CYP450, or peroxidases) to generate $o$-quinones. Various endogenous 1,2-benzene diols spontaneously generate $o$-quinones that can adduct to various biological molecules, including DNA. For instance, oxidized estrogen metabolites are linked to breast cancer $(311,312)$ by upregulating DNA damage through direct modification of DNA.(313)

Unlike most RES that are formed following ROS-initiated lipid-peroxidation events and carry messages downstream through protein modification, $o$-quinone-derived RES can additionally assist interconversion between different ROS forms. How the ROS-modulating properties of both $o$ - and $p$-quinones and their alkylating properties are linked to cancer is an ongoing area of debate.(200)

\subsubsection{Regulation of RES generation-Non-enzymatic generation of RES by ROS} acting on lipids (see section 2.2.1.2) can be considered as a means to preserve ROS signals, as RES tend to have longer diffusion distances than ROS (Table 1). The basal levels of 
various RES are maintained at low concentrations (Table 1). Oxidative stress as well as a number of pathological conditions promote RES generation.(91) Interestingly, HNE elevation can be caused by several stimuli, including cholesterol consumption.(314) Myocardial ischemia/reperfusion (I/R) injury significantly upregulates ROS generation. Analysis of myocardial tissue from mice following $30 \mathrm{~min}$ of ischemia and $30 \mathrm{~min}$ of reperfusion showed a $>15$-fold increase in levels of nitro-linoleic acid compared to shamoperated mice or mice that underwent only ischemia.(94) Pathological conditions associated with Alzheimer's and Parkinson's diseases (AD and PD) are also linked to increase in generation/accumulation of RES and RES-conjugated proteins. Brain tissues from patients with mild cognitive impairment and early AD show an increase ( 3-fold) in HNE and acrolein levels.(315) Furthermore, proteomic analysis of brain tissue reveals significant increase in carbonylated protein in $\mathrm{AD}$ and $\mathrm{PD}$ patients compared to age-matched controls. $(316,317)$

2.2.3 External sources of electrophiles-In addition to endogenous sources, cells are also continually exposed to non-native electrophiles. Exogenous sources include dietary electrophiles $(318,319)$ and environmental pollutants. Isothiocyanates are a family of dietary electrophiles produced by cruciferous vegetables such as broccoli and cabbage.

Isothiocyanates like sulforaphane and PEITC are activators of cytoprotective pathways(320) and have anticancer properties. $(321,322) \alpha, \beta$-unsaturated carbonyls like curcumin from turmeric(323) and cinnamaldehyde from cinnamon (324) also have protective effects on cells. Cells are also exposed to electrophiles from pollutants.(325) Acrolein is generated by heating oils at high temperature(306) and is also a major constituent of smoke from cigarettes(325) and exhaust fumes.(326)

\section{PHYSICOCHEMICAL PROPERTIES OF BIOLOGICAL RES AND ROS AND THEIR SENSORS IN TERMS OF INTRINSIC PROPERTIES AND REACTIVITIES}

In enzyme-assisted signaling/metabolic processes, enzyme participation can lead to reactions that are not expected based upon simple chemical principles. This can extend to exquisite and/or totally unexpected stereoselectivity, regioselectivity, and chemoselectivity (chemical-functional-group selectivity). Accordingly, the outcomes of these enzymemediated signaling processes are determined to a significant extent by enzymes. In enzymeindependent signaling modes, one may expect the stereoselection, regioselection, and chemoselection to be determined by the physiochemical properties of the signaling messengers. Consistent with this line of thought, cysteine is the primary target of both RES and ROS (high level of expected chemoselectivity), meaning that kinetic reactivity is certainly an important factor in this equation. However, we will also present several lines of evidence that shows that unexpected regio-/stereospecificity can be achieved by RES/ROS signaling, indicating that physicochemical properties of both the signaling small molecules AND target protein are important in redox signaling. The following sections will thus discuss the key physical characteristics of redox signals and what we know about the proteins that sense these reactive small molecules. In terms of the RES/ROS signal, we will discuss various physiochemical aspects that can favor reactivity. Although less is known 
about parameters/elements that render proteins susceptible to RES/ROS signals, we will address this key component at the end of the section.

The cell is unique because it is a strongly-reducing environment and hence finely tuned to respond to redox signals. Plasma is generally more oxidizing than the cell [redox couple is 60-90 mV more oxidizing than the cytosol; couple for cysteine/cysteine-cysteine-the

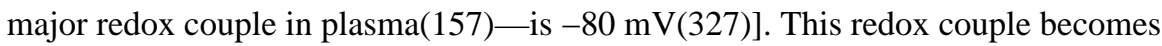
increasingly oxidative $(0.2 \mathrm{mV}$ per year) as organisms age or upon disease.(328) Unsurprisingly, oxidative damage to proteins has been reported to occur in plasma. For instance, oxidative modification of serum albumin (around 50-60\% total plasma protein content) occurs upon stroke in humans and rats.(329) However, the downstream ramifications of plasma oxidation are not as well-established as intracellular or direct contact cell-to-cell communications.

It is commonly assumed that a compound administered to the outside of a cell will quickly yield the same concentration within cells. This is not necessarily the case and even if equal distribution does eventually occur, establishment of equilibrium could take some time. Clearly, for reactive small molecules, equilibrium may be established particularly slowly because the cell will metabolize/react at a defined rate that may be similar to, or exceed, rate of import, leading to a lower intracellular concentration than the extracellular concentration. For oxidants that are actively metabolized by the cell, it is acknowledged that there is a gradient across the membrane. This was initially estimated to be 7-10 fold;(330) i.e., administration of $0.1 \mathrm{mM} \mathrm{H}_{2} \mathrm{O}_{2}$ will lead to a concentration of around $0.01 \mathrm{mM}$ in cells. More recent revisions have estimated that this value is closer to 700 fold. $(331,332)$ Clearly, care must be taken to define the range over which the specific gradient across the membrane is measured, as compensation factors may be saturated if extracellular $\mathrm{H}_{2} \mathrm{O}_{2}$ exceeds the detoxification capability of the cell, or apoptosis occurs. Finally, for reactive small molecules, especially RES and ROS, it is possible that reaction with serum proteins/buffers/ exogenous reactive molecules, such as thiols can occur prior to entry. This lowers the effective concentration of the small molecule, possibly in a time dependent manner.

Conversely, several reactive small signaling mediators, such as RES, can form long-lived (irreversible) adducts to various proteins, rendering diffusion across the membrane quasiirreversible. Thus, cellular "concentrative effects" have been established for numerous electrophiles, such as ITCs.(333) Thus: assume intracellular concentration of reactive small molecules or xenobiotics is the same as that in the media at your peril.

\subsection{Universal yardsticks with which to gauge concentrations, stabilities, and diffusion distances}

3.1.1 Concentrations and stabilities of canonical signaling particles in cells vary significantly and bear little relationship to intrinsic thermodynamic stability or concentration-ATP - the cellular energy storage device that is present at 1$10 \mathrm{mM}$ in cells - has a half-life of less than 60 minutes in the extracellular space(334) and this can drop to $0.2 \mathrm{~s}$ in the lung vasculature.(335) In cells, the half-life of ATP is on the order of seconds.(336) dATP on the other hand-a molecule with similar thermodynamic stability to ATP present in the cell at around one tenth of ATP concentration(337)—has a 
half-life of minutes in asynchronous cells.(338) Acyl phosphates (molecules that release similar energy to ATP upon hydrolysis) of murine prothymosin have a half-life of around 1 h.(339) Thioesters also have similar enthalpies of hydrolysis to ATP. However, half-lives of thioesters vary in cells significantly. For instance, the Ubc9-SUMO-1 thioester has a half-life of $3.6 \mathrm{~h}\left(k_{\text {hydrolysis }} 5.33 \times 10^{-5} \mathrm{M}^{-1} \mathrm{~s}^{-1}\right)$. On the other hand, $S$-palmitoylated Ras has a halflife between $20 \mathrm{~min}$ and $2 \mathrm{~h}$. (340)

Proteins also exhibit varied concentrations and half-lives, both of which are only weakly affected by protein size.(341) Most short-lived proteins in eukaryotes are degraded by the $26 \mathrm{~S}$ proteasome, a complex molecular machine consisting of a protease complex (20S) and a quality control/unfolding complex (19S). Ornithine decarboxylase is a classic short-lived protein with a half-life of around 20 min that is involved in various signaling pathways.(342) Ribonucleotide reductase (RNR) small subunit, a cell cycle regulated $45 \mathrm{kDa}$ protein important in DNA synthesis, has a half-life of $\sim 3 \mathrm{~h}$ in cells.(343) Ubiquitin-the small signaling polypeptide $(8 \mathrm{kDa})$ that ushers protein degradation through the ubiquitin proteasome pathway - has a half-life of approximately 10 h.(344) Longer-lived proteins [that are often degraded by autophagy(345)], such as RNR large subunit (90 kDa), purine biosynthesis enzyme, IMPDH (55 kDa), and Bcr-Abl (300 kDa; the oncogenic fusion protein responsible for various leukemias) have half-lives on the order of a cell cycle ( $20 \mathrm{~h}$ ). (346) Stabilities of proteins with post-translationally-modified residues can also vary widely. Phosphorylation has been recorded on histidine, serine/threonine and tyrosine. Phosphohistidine has a half-life on proteins between seconds(347) and 12 days.(348) Phosphate monoesters on the other hand have half-lives of $10^{12}$ years at room temperature. (349) Phosphatases can shorten the half-life of phosphorylated enzymes considerably.(350)

\subsubsection{Half-lives are critical components determining RES and ROS signaling} properties-Thus, half-lives vary significantly in the cell in a context-dependent manner. Half-lives do not particularly depend on the thermodynamic stability of the molecules in question, indicating that enzymatic regulation is the key determining factor. To some extent, the half-life of a molecule controls that molecule's ability to change concentration rapidly and dynamic range of response. This means that both magnitude of response as well as latency can be controlled by the ratio of synthesis: degradation rates. Rapidly-synthesized and rapidly-degraded particles can elevate concentration much more significantly for a given fold change in synthesis than a particle that is maintained at a similar concentration but is made and degraded slowly.(351) Similarly, a faster degradation rate will lead to a more rapid relaxation to basal levels once stimulation of production has decreased. Thus ultimately, rapid-response signaling particles tend to have rapid synthesis and turnovers. As we will see below, redox signals certainly fit into such a category.

3.1.3 Enzyme-assisted reaction rates-Standard Michaelis-Menten enzyme kinetics affords a second order rate constant $\left(k_{\mathrm{cat}} / K_{\mathrm{m}}\right)$ for reaction of enzyme with substrate. This value can be compared to the uncatalyzed second-order rate constant to show rate enhancements. For enzymes that have two substrates, such as glutathione-S-transferase (GST), we will use $k_{\text {cat }} / K_{\mathrm{m}}$ for the limiting reagent (such as HNE in the case of GST), provided the other substrate is close to saturation. GST has $K_{\mathrm{m}(\mathrm{GSH})}$ much lower than 
cellular glutathione concentration,(352) meaning that the conditions for calculation of $k_{\text {cat }} /$ $K_{\mathrm{m} \text { (HNE) }}$ (i.e., saturating GSH) is similar to conditions in a cell, at least in this respect. In general, for an average enzyme $k_{\mathrm{cat}} / K_{\mathrm{m}}$ is on the order of $10^{5} \mathrm{M}^{-1} \mathrm{~s}^{-1}$. Approximately $60 \%$ of all enzymes known fall within 2 orders of magnitude of this value.(353) $k_{\mathrm{cat}} / K_{\mathrm{m}}$ 's on the order of $100 \mathrm{M}^{-1} \mathrm{~s}^{-1}$ have been reported, although these are relatively rare.(354) Many enzymes with "low" $k_{\text {cat }} / K_{\mathrm{m}}\left(<1000 \mathrm{M}^{-1} \mathrm{~s}^{-1}\right)$ are secondary metabolic enzymes from plants, making relevance/identification of precise substrate somewhat questionable. Furthermore, low stability/misfolding of enzymes and potentially loss of positive regulators-parameters that are hard to assess experimentally—can reduce $k_{\text {cat }}$, rendering these values prone to underestimation; $K_{\mathrm{m}}$ tends to be a more reliable figure, as it is more or less independent of enzyme concentration, unless very high concentrations of enzyme are used, or the $K_{\mathrm{m}}$ is very low. The maximum theoretical $k_{\text {cat }} / K_{\mathrm{m}}$ is $10^{8}-10^{9} \mathrm{M}^{-1} \mathrm{~s}^{-1}$ although in practice this may not always be obtainable.

3.1.4 Diffusion distances-The diffusion distance for most redox signals is far below the length of the cell. However, since canonical signaling pathways can propagate the length of the whole cell and beyond, ultimately these "short range" signals can exert influence over a much longer range than their intrinsic diffusability suggests. However, for signaling to occur, the signal and primary sensor must lie within a suitably accessible distance, and so this section is concerned with signaling processes that occur when a native RES or ROS interacts with a specific sensor protein (first response signaling). The root mean square distance travelled by a diffusive reactive signal in 1D space is linked to half-life by the Einstein equation(355): $\mathrm{x}_{\mathrm{RMS}}=(2 \mathrm{D} t)^{0.5}$, wherein $\mathrm{D}$ and $\mathrm{t}$ designate diffusion coefficient (also see section 3.3.2) and time, respectively. Thus, as one would intuitively expect, longerlived or faster-moving reactive signals can cover more distance than shorter-lived, slower moving species. To promote "on-target" signaling, it is likely that the "choice" of signaling molecule in a specific pathway will be influenced by diffusion coefficient/stability. It is worth stressing that the values we discuss represent a threshold, i.e., a molecule of half-life $0.5 \mathrm{~s}$, with diffusion coefficient $\mathrm{D}=0.00001 \mathrm{~cm}^{-2} \mathrm{~s}^{-1}$, will travel $30 \mathrm{em}$ in one half-life. Thus "sphere of influence" is a function of size of the burst of signal produced, the threshold labeling required for signaling, and duration of the signal (i.e., length of time over which the signal remains in its intended location, a function of diffusability, stability, and time/duration of generation).

3.1.5 Dimensions of small molecules, proteins, and organelles-All diffusion distances must be evaluated in terms of the size of the cell and its components, so we describe some important distances below. Herein we compare linear dimensions (e.g., radius). However, in 3D space, differences scale with the cube of the radius, such that even small changes in radius correspond to large differences in volume. We start with the biological solvent, water, which has a diameter of $0.2 \mathrm{~nm}$. Single proteins range in size from around 5-8 kDa (e.g., SUMO and insulin) to over $300 \mathrm{kDa}$ (e.g., USP9x, approximately 300 $\mathrm{kDa}$; and titin, $3000 \mathrm{kDa}$ and $1000 \mathrm{~nm}$ long).(356) Proteins can also form large selfassembled structures such as the $26 \mathrm{~S}$ proteasome in eukaryotes (a $2000 \mathrm{kDa}$ conglomerate made up of $>30$ individual subunits). A rough rule of thumb states that a $100 \mathrm{kDa}$ globular protein will have a diameter of around $5-10 \mathrm{~nm}$. This dimension is close to the width of a 
lipid bilayer $(10 \mathrm{~nm})$. The ribosome has a diameter of $25-30 \mathrm{~nm}$ and mitochondria have diameters of $500 \mathrm{~nm}$. Aside from the nucleolus $(1000-3000 \mathrm{~nm})$, the nucleus $(10000 \mathrm{~nm})$ is typically the only intracellular structure large enough to be clearly resolvable by traditional light microscopy in cells. The typical nucleus:cytosol ratio for a cell is around $0.3-0.5$, although this can vary. The cell itself is usually $30,000 \mathrm{~nm}$ in diameter. However, some cells such as neurons can be considerably longer than this. How these size differences between cells and organelles affect redox signaling and its impact has not been systematically studied, but size differences may contribute to how impactful redox signaling is (as we discuss in section 3.7).

\subsection{Thiols are key players in RES/ROS communication and sensing}

\subsubsection{Cysteine is the most reactive of the canonical amino acid side chains; other residues may be reactive $-\mathrm{N}$-acetyl cysteine and glutathione are around 1000} times more nucleophilic than $\mathrm{N}$-acetyl histidine and $\mathrm{N}$-acetyl lysine (second-order rate constants for addition to HNE 0.0021 and $0.0013 \mathrm{M}^{-1} \mathrm{~s}^{-1}$, respectively). Alkylation of Nacetyl arginine by HNE is very slow, but its rate of alkylation by the more reactive electrophile ONE $\left(0.0006 \mathrm{M}^{-1} \mathrm{~s}^{-1}\right)$ is around 10-100 fold slower than that of $\mathrm{N}$-acetyl histidine and $\mathrm{N}$-acetyl lysine reacting with ONE.(90) Unsurprisingly, histidine, lysine, and to a lesser extent, arginine, have also been implicated as nucleophilic residues within proteins, although the rate data indicate that a significant rate enhancement or very high concentration will be required to outcompete cysteine (Figure 8).(357)

3.2.2 How cellular thiol pools regulate RES/ROS signaling-Cellular thiols are a nebulous "species" that are often discussed as a whole gamut. Separation between small molecule and protein thiols is also common in the literature. There is a tacit assumption that small-molecule thiols are "in abundance" in the cell. Indeed glutathione (the cell's principal small molecule reductant) is at "high concentrations", typically $1-10 \mathrm{mM}$ in whole cells. Reduced glutathione makes up around $98 \%$ of this value.(358) Some organelles such as mitochondria (which contribute 10-30\% of cellular glutathione) have similar concentration of glutathione (5-10 $\mathrm{mM}$ ) to the whole cell whereas the ER has a low content of reduced glutathione (25-50\% oxidized) but has higher total glutathione content (>15 mM).(98) What is often missed from the equation is the protein thiol content. Free protein thiol (as opposed to metal-bound, buried, or non-labile thiol, etc.) content is difficult to assess accurately. However, it is known that the concentration of total protein in a cell is around $0.2 \mathrm{~g} / \mathrm{ml}$.(359) Indeed, the total macromolecule content in a cell can be up to $30 \%$ total volume,(360) and this can be even higher in certain organelles/regions, such as the mitochondrial matrix $(60 \%)$. Assuming an average molecular weight of a protein is $50 \mathrm{kDa}$, the cellular concentration of total "protein" is at least $1 \mathrm{mM}$ (a reasonable figure since abundant proteins, such as ubiquitin and GST, can reach $50 \mu \mathrm{M}$ in cells).(359,361,362) With an average amino acid weighing $110 \mathrm{Da}$, and cysteines occurring in $2.26 \%$ of all amino acids within the human proteome, protein thiol concentration is approximately $10 \mathrm{mM}$. Protein thiols are thus equal to or exceed glutathione concentration.(363) There is reasonable experimental backing to this analysis. Using a thiol reactive dye, monobromobimane (Figure S3), Moldéus et al. showed that the cytosol contained $87 \mathrm{nmol}$ protein-thiol per mg protein [i.e., $20 \mathrm{nmol}$ of an average-size (50-kDa) protein].(364) Thus, on average, there are 4 
monobromobimane-reactive thiol equivalents per protein (leading to minimally $4 \mathrm{mM}$ total reaction protein thiol content). Perhaps most interestingly, this value dropped to 3 equivalents in the nucleus. Assuming that the average molecular weight of a protein is similar between the nucleus and the cytosol and that the dye is equally distributed between the nucleus and the cytosol, this result hints at that there is unequal nucleus:cytosol partitioning of thiol reactivity, either in terms of number or reactivity of thiols, or possibly reducing equivalents. There is certainly evidence that some small molecules do not readily exchange between nucleus and cytosol.(365)

It has been estimated that $80 \%$ of cysteines possess some functional importance.(366) This estimate is corroborated by the fact that cysteine shows an unusual conservation pattern in which it is either highly conserved or poorly conserved.(367) The term "functional importance" includes metal-coordinating cysteines. However some studies have shown that metal-binding cysteines can also be susceptible to reaction with electrophiles (likely when not bound to a metal).(368) It is thus at first glance surprising that less than $8 \%$ of reactive electrophile species administered to cells labels proteins.(369) This discrepancy can be explained by the fact that GSH conjugation to many xenobiotic electrophiles is enzymecatalyzed. Redox processes deactivating xenobiotics are also enzymatic. The enzymes that catalyze GSH-conjugation and redox processes can significantly increase rates of reaction over the uncatalyzed bimolecular rate constants. For instance, $k_{\mathrm{cat}} / K_{\mathrm{m}}$ for numerous GST isoforms indicates a 100-100000(352,370,371) fold increase in rate with respect to the uncatalyzed second order reaction between GSH and a Michael acceptor. Overexpression of GST reduces free HNE in cells(372) and extends lifespan in C. elegans,(373) whereas GSTknockout MEFs have elevated HNE levels.(374) However, how knockout of a particular GST isoform, GSTa4-4, affects HNE levels in mice depends on the strain used. In some strains, HNE elevation is observed,(375) whereas in others it is not and in these unaffected strains lifespan is also extended.(376) The uncatalyzed rate for GSH adduction to HNE is close to $1 \mathrm{M}^{-1} \mathrm{~s}^{-1}$. By means of comparison, carnosine (a histidine-containing dipeptide present in cells up to $150 \mathrm{mM})(99)$ can trap HNE in cells and protects cells against oxidative damage (Figure 8).(377,378) Based on the relative rate for adduction of histidine to HNE (0.001 with respect to GSH) and the concentration difference [1-10 mM (GSH) vs. up to $150 \mathrm{mM}$ (carnosine, providing histidine)], carnosine would not be expected to contribute much more than a tenth of the electrophiles trapped by cellular small-molecule thiols, even ignoring the action of GSTs.

There is considerable experimental evidence for protein labeling by endogenous oxidants and electrophiles; there is evidence that such sensing residues perform conserved functions; and there is also evidence that ROS may have shaped evolution of several genes, including Nrf2. It is also possible that "ROS processing" may have shaped organelle evolution.(379381) There are five principal mitigating factors that enable labeling of target proteins in the presence of the arsenal of cellular small-molecule nucleophiles: (I) high levels of ROS may cause depletion of GSH/inhibition of detoxification enzymes(382) [either local(383) or global;(384) likely a significant component of protein labeling under bolus RES/ROS dosing conditions]; (2) the cell can suppress its ability to process reactive species, allowing buildup; (385) (3) the cell can cause local release of high concentrations of a RES/ROS molecule; (386-388) (4) some specific proteins are endowed with kinetically "privileged" cysteines 
that can compete with detoxification processes to gain sufficient occupancy to elicit downstream signaling;(389) and (5) modification of such sensor proteins elicits phenotypically-dominant signaling outputs which would ensure coordinated responses "specific" to the target protein.(251) Although it is not known how all these mechanisms fit together, nor how important each one is, it is our opinion that biology has likely harnessed all means at its disposal to bias target-protein-specific signaling (note: since thresholds of occupancy may differ between "intended target" and off target proteins, this is not necessarily the same as target-specific labeling).(251)

Gradients of $\mathrm{H}_{2} \mathrm{O}_{2}$ across the cell help to promote intended protein labeling and possibly help trigger specific signaling factors(382). However, whether such gradients are sufficient to allow specific signaling events is a much harder question to answer. It is likely that privileged sensors have enzyme-like reaction efficiencies with RES/ROS signals they sense and respond to.(390) For instance, Keap1, a multipurpose sensor of redox signals has a second-order rate constant with peroxide of approximately $140 \mathrm{M}^{-1} \mathrm{~s}^{-1}$; for the same reaction, GAPDH has a second-order rate constant of $500 \mathrm{M}^{-1} \mathrm{~s}^{-1}$. These rates are of orders of magnitude higher than cysteine adducting to peroxide.(66) Nevertheless, assuming 50\% occupancy is required for downstream signaling, it has been calculated that $170 \mu \mathrm{M} \mathrm{H}_{2} \mathrm{O}_{2}$ for $30 \mathrm{~s}$ would be required to usher downstream signaling by Keap1.(66) Clearly, phenotypic dominance lowers the threshold for activation, shortening the required exposure time or concentration required to elicit signaling.

\subsubsection{Protein targets are most likely the key determinant of RES/ROS}

bioactivity-From the discussion above, based on total concentrations as well as the efficiency of GSTs, glutathione pool depletion is one of the first casualties following bulk exposure to exogenous RES/ROS. Thus, significant effort has been invested to understand whether depletion of the GSH-pool or modification of proteins is the primary source of bioactivity of reactive small molecules. Although it is very difficult to parse these events in most systems, the general consensus is that proteins are the principal biologically-relevant target of electrophiles in cells especially under signaling conditions as opposed to oxidative damage, $(391,392)$ although there remains some debate. $(393,394)$ Several lines of evidence exist for proteins being the principal signaling targets of ROS/RES. Accumulating evidence suggests that RES trigger specific response by direct modification of specific proteins. $(251,252,259)$ Using mutagenesis, it has been shown that sulfenic acid formation is required for several signaling events in cells and whole organisms. For instance, IRE-1-mediated stimulation of SKN-1 signaling in $C$. elegans is governed by oxidation of a single cysteine. (395) Depletion of GSH by butathione sulfoximine (an inhibitor of GSH synthesis) is more significant than PEITC treatment and further induces ROS to a higher level than PEITC. However, PEITC is a much better inducer of apoptosis than butathione sulfoximine.(396) Consistent with proteins being the target of PEITC, knockdown of putative target proteins sensitizes cells to PEITC.(333) Modification of specific thiols by HNE has also been linked to synaptotoxicity,(397) AR,(398) and kinase inhibition(399) amongst others. In the last example, signaling was ascribed to a specific cysteine, loss of which ablated signaling, proving that signaling was protein specific. We will discuss more about this in section 5.2, but we focus in this present section mainly on how ROS and RES interact with proteins. 


\subsection{Diffusion distances and half-lives}

3.3.1 Caveats with calculating half-lives/diffusion distances-Most researchers

have used model systems and in vitro analyses to understand diffusion distances. These analyses are informative and can provide a basis/foundation for a theoretical understanding of redox signaling/signal transduction, but the data must always be viewed with caution. In the past 15 years, more efforts have been made to understand ROS/RES in cells. Although cellular data are more informative about distances traveled etc., differences in stability between the specific ROS in question in water, or an approximation of the cytoplasm and the cell, can give an indication of how significantly ROS interacts with cellular components. Unfortunately, many of the values quoted differ over almost ten-fold between different reports, different cell types, and/or experimental conditions. This margin of error can be the difference between concluding that the specific ROS mainly reacts with solvent and mainly interacts with the cellular milieu. Most estimates of diffusion distance rely upon the Einstein equation shown above (section 3.1.4).(355)

\subsubsection{Diffusion coefficients are hard to calculate in cells-One of the key}

parameters in the Einstein equation is the diffusion coefficient, D. This constant is dependent upon several parameters including viscosity (D a $1 / \eta$ ). The relative viscosity of water compared to the cytoplasm has been measured to be between 1.6 and 1.1 using various methods including rotational diffusion coefficient of GFP and fluorescence correlation spectroscopy. $(400,401)$ However, some experiments have suggested that the relative viscosity of cells is 4 fold higher than water, depending on the size of protein measured. (402)

3.3.2.1 There is no good model for the cellular milieu in vitro: The high concentrations of total protein found in cells are only obtainable in vitro under specific conditions, such as protein crystals (where 50\% of the crystal is usually protein).(403) Thus, it is hard to convert in vitro data to numerically relevant data in a cell. In addition to protein concentration, the predominance of lipid membranes within a cell sets up a series of complex, non-classical environments. For instance, the surface area of ER and mitochondrial membranes alone in 1 $\mathrm{mL}$ of liver tissue covers an area close to $20 \mathrm{~m}^{2}$.(404) On the other hand, an increasingly well-appreciated part of the oncogenic program is elevation of the overall intercellular $\mathrm{pH}$. Redox modifications are $\mathrm{pH}$ sensitive,(405) indicating that redox signaling itself may differ between healthy and diseased cells.

3.3.2.2 The cell is compartmentalized: Compartmentalization is a fundamental property of living systems: even in E. coli, some proteins localize to specific regions/loci/poles.(406) In eukaryotes, this is even more complex due to membrane compartmentalization. Different membrane vesicles can have widely different $\mathrm{pH}$, metal-ion content, or reducing capacity with respect to the cytosol. Such variables intrinsically affect the stabilities/spontaneous generation rates/interconversion rates of RES/ROS. For instance, $\mathrm{pH}$ rate profiles for reaction of cysteine and peroxide indicate two important points of inflection at $\mathrm{pH}=8.5$ and 11.5. These values correspond to the $\mathrm{p} K_{\mathrm{a}}$ 's of cysteine sulfur and hydrogen peroxide. Thus, one would expect cysteine oxidation by peroxide to be considerably slower in a lysosome(407) [a small organelle, less than $1 \mu \mathrm{m}$ in size, with $\mathrm{pH} 4.5-5.0$, known to contain 
free cysteines(408)] than the cytoplasm (pH 7.2). However, proteins can perturb $\mathrm{p} K_{\mathrm{a}}$, allowing access to protonation states of residues typically inaccessible at neutral $\mathrm{pH}$. For instance, the nucleophilic thiol of human $\operatorname{Prx} 5$ has a $K_{\mathrm{a}}$ of $5.5 ;(409) \mathrm{p} K_{\mathrm{a}}$ of the catalytic lysine within acetoacetate decarboxylase was measured by Westheimer to be around 6.(410)

3.3.2.3 Enzymatic clearance can determine cellular half-life: Enzymes can accelerate rates of reaction by up to 17 orders of magnitude,(411) although such high accelerations are reserved for particularly difficult transformations. Cells express proteins at widely-different levels, and different isoforms of a specific enzyme (that can have widely different specific activities and/or functions) can be expressed in a cell type-dependent manner. Since enzymecatalyzed metabolism is the predominant clearance mechanism for many small molecules in cells, it stands to reason that diffusion distance of reactive signals through cells is a function of metabolic protein expression. Thus, diffusion distances are highly cell type- and localedependent. For instance, some cell types, such as the human breast cancer line, MCF7, display very little GST activity,(412) possibly increasing diffusion distances of redox signals in this line, although this has not been investigated.

3.3.2.4 Which ROS?: Many half-life measurements of various chemotypes of ROS have been carried out using a sensor that changes properties upon reaction with a type of ROS. These sensors will be discussed in section 5. Of course, these sensors may not be absolutely faithful in their ability to report on the specific ROS in question. Such intricacies are particularly important in a cell where even a single ROS can subsequently generate numerous secondary ROS and there are numerous compartments with different properties that can affect signaling. Similarly, many of the methods used to estimate ROS half-lives involve generation of ROS in situ. It may not always be known how the generation methods affect the cell or produce side products (especially in cells).

\subsubsection{Diffusion distances}

3.3.3.1 ROS are relatively short-lived: In general, ROS are shorter-lived than RES (Table 1). The most common ROS are (in order of increasing cellular half-life) ${ }^{\bullet} \mathrm{OH}, \mathrm{O}_{2}{ }^{\bullet-},{ }^{1} \mathrm{O}_{2}$, and $\mathrm{H}_{2} \mathrm{O}_{2}$. Nitrated forms of ROS, such as $\mathrm{ONOO}^{-}$may also contribute to redox signaling. Interestingly, biological ROS species are physicochemically divergent, conferring a wide range of possible protein modifications. ${ }^{1} \mathrm{O}_{2}$ is unique in the sense that it is a metastable excited electronic state of ${ }^{3} \mathrm{O}_{2}$. Thus, ${ }^{1} \mathrm{O}_{2}$ decays (via phosphorescence, the so called monomol emission)(413) to ${ }^{3} \mathrm{O}_{2}$ at a defined rate in the absence of a reactive residue/ enzyme/quencher. This process sets an intrinsic upper limit on the lifetime of ${ }^{1} \mathrm{O}_{2}$ and has been used to monitor ${ }^{1} \mathrm{O}_{2}$ in cells (see section 5).

3.3.3.2 Diffusion distance of hydroxyl radical: ${ }^{\circ} \mathrm{OH}$ is the most reactive of common ROS. Unsurprisingly, this has the most favorable reduction potential of all common forms of ROS $(2.3 \mathrm{~V})$. It is believed to react at close to diffusion-limited rates with numerous substrates $\left(10^{9}-10^{10} \mathrm{M}^{-1} \mathrm{~s}^{-1} ;(68,69)\right.$ e.g., second-order rate constant with $\left.\mathrm{GSH}=2.3 \times 10^{10} \mathrm{M}^{-1} \mathrm{~s}^{-1}\right)$. The diffusion distance is around $1-5 \mathrm{~nm}$ (i.e. less than the width of a $100 \mathrm{kDa}$ protein). $(67,414)$ This radical can be formed from several more stable ROS (including $\mathrm{H}_{2} \mathrm{O}_{2}, \mathrm{O}_{2}{ }^{--}$ and $\mathrm{ONOO}^{-}$), and thus may have a larger effective diffusion distance than suggested in most 
tables. ${ }^{\circ} \mathrm{OH}$ can initiate fragmentation of several amino acids that can initiate other signaling cascades. One interesting case is ${ }^{\circ} \mathrm{OH}$-oxidation of methionine,(415) which releases methanethiol,(416) a molecule with possible signaling roles. The byproduct of these fragmentation processes is typically a carbon-centered radical. These product radicals can trap ${ }^{3} \mathrm{O}_{2}$ to form ${ }^{\bullet} \mathrm{OOH} / \mathrm{O}_{2}{ }^{--}$. Thus ${ }^{\bullet} \mathrm{OH}$ can attack protein targets, modify their primary structure, and create in the process subsequent radicals that can usher secondary signaling processes. Owing to dative stabilization from the appended oxygen, ${ }^{\circ} \mathrm{OOH}$ and ${ }^{\circ} \mathrm{OOR}$ have lower reduction potentials than ${ }^{\circ} \mathrm{OH},\left({ }^{\circ} \mathrm{OR}, \mathrm{E}^{0}=1.6 \mathrm{~V} ;{ }^{\bullet} \mathrm{OOH}, \mathrm{E}^{0}=1.1 \mathrm{~V} ;{ }^{\bullet} \mathrm{OOR}, \mathrm{E}^{0}=1.0 \mathrm{~V}\right)$. (417) These oxygen radicals likely have signaling roles, although how much they differ from $\cdot \mathrm{OH}$ is unknown.

3.3.3.3 Diffusion distance of superoxide: $\mathrm{O}_{2}{ }^{--}$can spontaneously decay through dismutation. However, this reaction is complex. The spontaneous rate for dismutation of two $\mathrm{O}_{2}{ }^{--}$(anionic) molecules is very slow, $(418,419)$ meaning one or more of the molecules must be protonated for dismutation to occur, rendering the rate $\mathrm{pH}$ dependent. At $\mathrm{pH} 7$, with micromolar $\mathrm{O}_{2}{ }^{--}$, the half-life of dismutation is approximately $10 \mathrm{~s}$ (indicating that $\mathrm{HO}_{2}{ }^{\bullet}$ is accessible at this $\mathrm{pH}$ ).(418) Additionally, as spontaneous dismutation is a homo-bimolecular process, at low concentrations, half-life of $\mathrm{O}_{2}{ }^{--}$can extend to hours. $\mathrm{O}_{2}{ }^{--}$reacts with thiols in a complex reaction. The reported second-order rate constants for thiol reaction vary by 4 orders of magnitude (15 to $\left.10^{5} \mathrm{M}^{-1} \mathrm{~s}^{-1}\right)$. $(420,421)$ Although different small-molecule thiols can likely adduct superoxide at different rates, even for a specific thiol such as GSH, the literature rates for adduction to superoxide range over several orders of magnitude (200-10 $\left.\mathrm{M}^{-1} \mathrm{~s}^{-1}\right) .(204,422)$ Overall, it is likely thiol adduction to superoxide is not much more efficient than dismutation. As we discussed above, SOD is principally responsible for metabolism of $\mathrm{O}_{2}^{--}$. The diffusion distance of $\mathrm{O}_{2}^{--}$in the presence of SOD is around 0.5 $\mu \mathrm{m}$.(59) Unsurprisingly, basal levels of $\mathrm{O}_{2}{ }^{--}$in cells are low $\left(10^{-9}-10^{-12} \mathrm{M}\right)$.(58) It is important to note that different organelles have different steady state ratios of $\mathrm{O}_{2}{ }^{--}: \mathrm{H}_{2} \mathrm{O}_{2}$. For instance, in the cytosol this ratio is 1:1000; it is 1:100 in mitochondria.(423)

3.3.3.4 Diffusion distance of ${ }^{1} \mathrm{O}_{2}$ : With the onset of photodynamic therapy, ${ }^{1} \mathrm{O}_{2}$ in cells has grown in importance.(77) As outlined above, ${ }^{1} \mathrm{O}_{2}$ can spontaneously decay to ${ }^{3} \mathrm{O}_{2}$, in the process emitting energy $(1270 \mathrm{~nm})$.(424) Using this unique trait, and taking advantage of the fact that the lifetime of ${ }^{1} \mathrm{O}_{2}$ can be extended in $\mathrm{D}_{2} \mathrm{O}$, the cellular lifetime of ${ }^{1} \mathrm{O}_{2}$ was calculated to be $3 \mu \mathrm{s}$. This is close to the inherent upper lifetime of $4 \mu \mathrm{s}$ in water(74) (compare $46 \mu \mathrm{s}$ in $\mathrm{D}_{2} \mathrm{O}$ treated cells, versus $68 \mu$ s in $\mathrm{D}_{2} \mathrm{O}$ ). $(76,78)$ This result indicates that the root mean square displacement of ${ }^{1} \mathrm{O}_{2}$ in water is $270 \mathrm{~nm}$. Given that these data are similar to quenching in water, it is likely that solvent quenching dominates ${ }^{1} \mathrm{O}_{2}$ half-life/ diffusion in bulk cells. Importantly, however, half-life of ${ }^{1} \mathrm{O}_{2}$ can be extended up to $14 \mu \mathrm{s}$ in inert lipids, $(77,425)$ meaning that ${ }^{1} \mathrm{O}_{2}$ has more time to interact with cellular components when it is in membranes. Consistent with ${ }^{1} \mathrm{O}_{2}$ interacting strongly with cellular milieu, other estimates of stability of ${ }^{1} \mathrm{O}_{2}$ have indicated that this molecule has about 100 to 10 - fold lower stability than expected based upon solvent reaction in cells. $(426,427)$ Trivial explanations for these discrepancies are differences in cell lines and detection methods.(428) Additionally, second-order rate constants for reaction of ${ }^{1} \mathrm{O}_{2}$ with methionine or cysteine/GSH are sizeable (approximately $10^{7} \mathrm{M}^{-1} \mathrm{~s}^{-1}$ )(82) meaning that stability could be 
affected by cellular reductants and competing nucleophiles that could be cell type/region specific. It is also noteworthy that ${ }^{1} \mathrm{O}_{2}$ cannot be added directly to a cell. It is typically generated in situ by photosensitization and often read out indirectly. In this respect, phosphorescence may serve as the most direct measurement of ${ }^{1} \mathrm{O}_{2}$.

Endogenous ${ }^{1} \mathrm{O}_{2}$ is most likely formed in light-exposed areas, such as the skin. Sensitizing of ${ }^{3} \mathrm{O}_{2}$ by a range of excited biomolecules to generate ${ }^{1} \mathrm{O}_{2}(44)$ may have a role in skin cancer etiology and aging.(429) Consistent with this notion, direct observation of ${ }^{1} \mathrm{O}_{2}$ formed by action of UVA-light on pig and human skin has been detected using phosphorescence (1270 nm).(430)

3.3.3.5 Diffusion distance of peroxide: Peroxides, including organic peroxides, have the longest diffusion range of all ROS. Hydrogen peroxide is stable in water and is commercially available. Disulfide formation via the reaction of cysteine with peroxide in water is formally a two-step process: the first step is nucleophilic attack of free cysteine on peroxide to form a sulfenic acid and water, the second step involves displacement of the product sulfenic acid by another cysteine. The first step occurs with a second order rate constant around $1 \mathrm{M}^{-1} \mathrm{~s}^{-1}$ around neutral $\mathrm{pH} .(111,431)$ The second step is over one order of magnitude faster and likely does not contribute to the kinetics in vitro.(432) Assuming a cellular thiol content of $5 \mathrm{mM}$, and given the $1 \mathrm{M}^{-1} \mathrm{~s}^{-1}$ second order rate constant for thiol adduction to peroxide, one would expect a half-life of around $2 \mathrm{~min}$ in cells. However, the half-life of peroxide in a cell has been calculated to be between $10^{-3}$ and $10^{-5} \mathrm{~s}$.(24) Thus, enzyme catalyzed pathways dominate peroxide metabolism. Indeed, assuming that peroxiredoxin [that has a second-order rate constant of $10^{8} \mathrm{M}^{-1} \mathrm{~s}^{-1}$ with peroxide $(109,111)$ ] is the dominant peroxide quencher in the cell (and that this enzyme is maintained in the reduced state) the longest diffusion distance for peroxide in a cell is $1 \mu \mathrm{m}$.(65) Thus, peroxide metabolism contrasts with the decay mechanisms of ${ }^{1} \mathrm{O}_{2}$ and ${ }^{\circ} \mathrm{OH}$, which are (likely) dominated by solvent quenching, at least in aqueous environments. This line of reasoning further suggests that peroxide stability and diffusion distances may be cell-type/ context dependent.

\subsection{ROS-metal complexes can alter physicochemical properties}

Many ROS can interact with numerous metals and metalloproteins. In addition to low molecular weight complexes, reaction of peroxide with metalloproteins can also alter ROS properties. For example, reaction of $\mathrm{H}_{2} \mathrm{O}_{2}$ with heme peroxidases, such as MPO and lactoperoxidase, generates a two-electron oxidized Compound I species (similar to Compound I species in CYP450 discussed in section 2.1.2.3). Compound I oxidizes various substrates to generate oxidants such as $\mathrm{HOCl}$ and free radicals.(112,433) It is likely that reaction with free iron dominates in cells (434) because the labile iron pool [concentration $0.6 \mu \mathrm{M}$ in lymphocytes(435) and $0.2-1.5 \mu \mathrm{M}(436)$ in resting erythroid and myeloid cells] dwarfs other redox-active metal species. However, this is probably cell-type/organism/ condition/organelle-dependent.(437) The reaction of peroxide with ferrous iron [Fe(II)] to create the ${ }^{\bullet} \mathrm{OH}$ is called the Fenton reaction. This process generates ${ }^{\circ} \mathrm{OH}$ with a rate constant on the order of $100 \mathrm{M}^{-1} \mathrm{~s}^{-1}$.(438) ${ }^{\bullet} \mathrm{OH}$ also reacts with Fe(II) (rate constant $3.2 \times 10^{8} \mathrm{M}^{-1} \mathrm{~s}$ ${ }^{-1}$ ), but since ${ }^{\bullet} \mathrm{OH}$ is inherently unstable, this process is believed to contribute little to the 
stability of ${ }^{\bullet} \mathrm{OH}$. The iron- $-^{\bullet} \mathrm{OH}$ reaction is only an order of magnitude faster than the reaction of ${ }^{\bullet} \mathrm{OH}$ with peroxide $\left(3.3 \times 10^{7} \mathrm{M}^{-1} \mathrm{~s}^{-1}\right)$, for example. Protein-bound iron can also catalyze Fenton-type processes. However, as the Fenton reaction proceeds via an $\mathrm{Fe}(\mathrm{II})$ state, not all iron-containing metalloproteins can catalyze this sort of chemistry. $(439,440)$ Redoxactive metals can also catalyze dismutation of $\mathrm{O}_{2}{ }^{-\boldsymbol{}}$, but this is overall a similar process to the enzyme-catalyzed reaction we have previously discussed (section 3.3.3.3) and likely is less impactful than the effect on $\mathrm{H}_{2} \mathrm{O}_{2}$.

\subsection{RES are long lived and have long diffusion distances}

Many RES are formed by lipid peroxidation or oxidative metabolic pathways and so they are intrinsically linked to ROS. Several metabolic byproduct RES [e.g. $30 \mu \mathrm{M}$ formaldehyde (441)] are prevalent in the blood. It has recently been shown that a major source of formaldehyde is folate, and it has been delineated how formaldehyde can be diverted to one carbon metabolism.(442) Methylglyoxal, another metabolic side product and arguably the second least chemically complex RES after formaldehyde, is also relatively abundant. In cells this molecule can reach up to $300 \mu \mathrm{M}$, although much of this is bound in cells (for instance $0.1-2 \%$ of arginine residues in cells are modified by methylglyoxal).(443,444)

Arguably, the more chemically diverse and signaling relevant class of RES is RES derived from lipid peroxidation. The simplest unsaturated aldehyde electrophile is acrolein. This reacts with GSH with a rate constant of $120 \mathrm{M}^{-1} \mathrm{~s}^{-1}$.(445) 3-Substitution on the enal typically decreases electrophilicity and such electrophiles undergo conjugation to GSH with second-order rate constants between 0.3 and $3 \mathrm{M}^{-1} \mathrm{~s}^{-1}$.(446) The presence of a $\gamma$-hydroxyl group on the Michael acceptor HNE promotes nucleophilic addition by $1.5-10$ fold $(85,447)$ relative to the dehydroxy congener, nonenal, likely through a combination of increase in positive charge and anti-periplanarity of the $\mathrm{C}-\mathrm{O}$ bond and nucleophile in the transition state.(448) Oxidation of the hydroxyl group to a ketone hugely increases electrophilicity,(85) possibly through a "dual attraction" mechanism.(449) 4-Oxononenal (ONE) $\left(k_{\mathrm{GSH}}=145 \mathrm{M}\right.$ ${ }^{-1} \mathrm{~s}^{-1}$ ) is significantly more reactive than HNE, although this molecule is likely at significantly lower concentrations than HNE.(450) HNE and malondialdehyde each account for $20 \%$ of lipid peroxidation products.(451) HNE has been estimated (after subtraction of malondialdehyde) to be present in K562 cells at a concentration of approximately 100 $\mathrm{pmol} / \mathrm{mg}$ protein, corresponding to a concentration of around $8 \mu \mathrm{M}$.(84) High-energy UVlight doubled this value. However, other estimates indicate a lower basal level of HNE (0.3$1 \mu \mathrm{M})$, but a much larger response upon stress, up to $5 \mathrm{mM}$.(85)

HNE is estimated to have a half-life of around 2.5 min given the known concentration of GSH (5 mM) in liver cells, (85) the known rate of adduction of GSH to HNE and assuming there are no additional factors promoting adduction, like catalysis. Interestingly, HNE has a half-life of less than $4 \mathrm{~s}$ in rat heart(85) and around $30 \mathrm{~s}$ in hepatocytes suspensions.(88) The discrepancy between calculated stability and actual stability can be ascribed to enzymatic catalysis.(452) Consistent with this analysis, perturbation to principal detoxifying enzymes like mitochondrial aldehyde dehydrogenase increases cardiac HNE levels.(453) The percentage of HNE incorporated into protein is dependent upon the concentration of HNE added in hepatocytes. $100 \mu \mathrm{M}$ led to $3 \%$ incorporation; $1 \mu \mathrm{M}$ led to $0.62 \%$ incorporation. 
(88) Overall this agrees with other reports showing that 1-8\% of HNE labels proteins in cells. Of the remaining 99-92\% HNE, oxidation/reduction and GSH conjugation accounts for around 50\% each, although estimates for this partitioning vary.(454) In spite of the reliance on glutathione to detoxify HNE, even upon treatment with $250 \mu \mathrm{M} \mathrm{HNE}, 30 \%$ of reduced glutathione (i.e. unreacted) is retained in hepatocytes.(455) Where the data are known, nitro-olefin metabolism seems to be similar to LDE metabolism: nitro-olefins have a short half-life (minutes) in plasma.(456) Importantly, however, nitro-olefins can remain bound to protein, and be released depending on the environment (e.g. pH change). It is thus generally true that RES are longer lived than any ROS in cells, meaning that these signals have different latencies and can diffuse much further.

\subsection{Both ROS and RES are membrane diffusible}

Most uncharged species can permeate through the cell membrane.(255) For this reason, $\mathrm{O}_{2}{ }^{\cdot-}$ is the least permeable common ROS/RES. This argument assumes that $\mathrm{O}_{2}{ }^{--}$is considerably deprotonated at neutral $\mathrm{pH}$, which may not entirely counterbalance the reactivity of the protonated state.(457) For instance, protonation of $\mathrm{O}_{2}{ }^{--}$can increase the spontaneous dismutation rate by at least 5 orders of magnitude. Furthermore, although the ratio of unprotonated to protonated species is around 1000:1 at $\mathrm{pH} 7.8,(458)$ the equilibrium lies close to unity around $\mathrm{pH} 4.5$ (a value obtainable in acidic organelles). In some cases, there is evidence that $\mathrm{O}_{2}{ }^{--}$can pass through membrane channels, (459) most notably anion channels(460), although there seems to be some other mechanism(s) that enable superoxide to pass through membranes.(461) $\mathrm{H}_{2} \mathrm{O}_{2}$, a product of dismutation, is accepted to be membrane permeable. $(387,462)$ Indeed, peroxide can behave as a secondary messenger for $\mathrm{O}_{2}{ }^{-2}$ ferrying extracellular $\mathrm{O}_{2}{ }^{--}$signals to the cytosol. The abundance of extracellular SOD also assists in this process by localizing peroxide to the point of origin.(59) $\mathrm{H}_{2} \mathrm{O}_{2}$ likely enters the cell through aquaporins.(463) Because the membrane is a different environment to the cellular milieu, it can also affect stability of some ROS. Diffusion distance of ${ }^{\circ} \mathrm{OH}$ is less than the width of the cell membrane; thus, it is unlikely to be able to cross this barrier. Nonetheless ${ }^{\bullet} \mathrm{OH}$ can certainly cause damage to membranes.(271) ${ }^{1} \mathrm{O}_{2}$ and ${ }^{3} \mathrm{O}_{2}$ appear to have different permeabilities to the membrane, even though the lower limit of ${ }^{1} \mathrm{O}_{2}$ diffusion rate through a membrane is similar to ${ }^{3} \mathrm{O}_{2}(2 \mathrm{~cm} / \mathrm{s})$. (464) It has thus been postulated that ${ }^{1} \mathrm{O}_{2}$ is more affected by molecules that can quench or react with it in the membrane than ${ }^{3} \mathrm{O}_{2}$. This discrepancy is not unexpected as ${ }^{1} \mathrm{O}_{2}$ can undergo several pericyclic reactions with unsaturated lipids. As they share characteristics of lipids, RES are usually considered to be highly membrane permeable.

\subsubsection{ROS diffuse rapidly, compared with macromolecule diffusion, especially}

in cells-Diffusion is more difficult in the cell than in water.(465) This has been shown for small molecules using a range of techniques from pulsed field $\operatorname{NMR}(466,467)$ to photobleaching.(468) Reduced diffusion rates in cells can be attributed to a number of factors including, interaction with proteins, various aspects of surface chemistry and crowding.(469)

Macromolecules diffuse slower than small molecules in water: this effect is dependent on size with larger macromolecules diffusing slower than smaller ones.(416) Although there 
have been disagreements,(470) the differences in diffusivity likely apply to proteins in cells too. For instance, across five proteins spanning $21-540 \mathrm{kDa}$, the diffusion coefficients decreased 5-fold.(467) Interestingly, the same study showed that the difference between diffusivity in water and the cytosol increases as the size of the protein increases. Recent work using fluorescence cross-correlation spectroscopy(402) agreed that larger proteins show severely slowed diffusion and stressed the heterogeneity of cells as a causative factor. (471) The cell thus appears to present a "random obstacle network" to species diffusing through it. Interestingly, this study also concluded that the nucleus and cytosol were similarly permissive to diffusion.

Unlike many small molecules (including redox signals), macromolecules cannot diffuse across membranes, meaning that movement from one organelle to another is regulated. For instance, nuclear pores are believed to allow molecules below a specific size to cross from cytoplasm to nucleus unhindered (see section 3.2.2 for some possible exceptions). It is often said that proteins above $60 \mathrm{kDa}$ require import. However, reports also claim that molecules larger than this can enter the nucleus freely.(472) Above the cut-off (whatever the criteria be), recognition is required for nuclear entry. Entry of "large" molecules is facilitated by a series of protein chaperones called importins. These proteins recognize specific sequences allowing import into the nucleus. Exportin, on the other hand, mediates nuclear export. Similar complex sorting processes happen in other organelles. Since proteins diffuse slowly and require chaperoning across membranes, papers discussing redox "communication" between organelles have grown in abundance over the recent years and were originally discussed in section 2.1.3.

\subsection{Manifestation of diffusion distance differences as a function of cell size}

The need for rapid information transfer under stressed circumstances may have spurred biology to harness small-molecule messenger systems such as redox signaling. Depending on the cell type (E. coli, epithelial, axon), it can take a protein between $10 \mathrm{~ms}$ to 20 days to diffuse from one side of the cell to another. Furthermore, from prokaryotes to eukaryotes the average length of a protein in amino acids increases $50 \%$, possibly indicating that diffusivity of proteins decreases as genome complexity increases.(473) Based on this logic, the rapid diffusion properties associated with redox signals likely become more desirable relative to macromolecular systems as the cell increases in size/complexity and diffusion times for macromolecules increase.(474) Thus, eukaryotic cells may be more dependent on redox signaling than prokaryotic cells. There is certainly evidence that E. coli peroxiredoxin and mammalian peroxiredoxin (a principal mediator of ROS levels and hence ROS signaling) are regulated differently by ROS, indicating that responsivities to redox signaling are not the same in mammals and prokaryotes.(383)

Indeed, gram-positive prokaryotes and eukaryotes use different redox couples: intracellular redox buffer in mammalian cells is GSH, whereas bacteria can use a range of different reductants aside from GSH,(475) including acetyl CoA,(476) mycothiol,(477) and bacillithiol.(478) Thus, how proteins are modified under oxidative stress and resulting conformation changes and re-reduction dynamics are likely very different between these two domains of life. 


\subsection{Organelle-specific exposure by RES/ROS under bolus dosing}

$\mathrm{HNE}$ and $\mathrm{H}_{2} \mathrm{O}_{2}$ permeate cells differently under bolus dosing: external $\mathrm{HNE}$ treatment of cells labels both cytoplasm and nucleus whereas external treatment of cells with $\mathrm{H}_{2} \mathrm{O}_{2}$ affects only the cytoplasm.(479,480) Analogous experiments using a ROS sensing fluorescent protein called "HyPer" have reached a similar conclusion that the nucleus is relatively more protected than many organelles to ROS. Although there could be many explanations for this result (see also section 3.2.2), there is a clear indication that external RES and ROS can affect organelles differently.(481)

\section{9 (Ir)Reversibility of RES/ROS-derived protein modifications}

Reversibility of signaling processes, and the lifetimes of specific modifications are of critical importance. Taking analogy from the drug discovery community, it is clear that the longer a drug (or redox signal) resides on its target, the more impact the signal can have. For a drug, long residency will typically be ideal. However, (as discussed above) for signaling, flexibility-i.e., the ability to turn on and off (with minimum energy expenditure) - is likely most desirable. Moreover, for reversible signals, relatively little effort is required to turn on and off the signal, whereas degradation is required to suppress the signal if the protein is irreversibly modified (requiring more of the cell resources). Regardless of reversibility, some oxidation events spawn further downstream signaling processes, often through involvement of enzymatic or glutathione-mediated reduction, or through trapping of ${ }^{3} \mathrm{O}_{2}$. We discuss these nuanced points in this section.

\subsubsection{Sulfur oxidation states show differing reversibility in cells-Cysteine}

oxidation to sulfenic acid/disulfides is freely reversible. Sulfenic acid reduction can be either enzymatic [glutaredoxin and thioredoxin systems are particularly efficient and allow rapid enzymatic re-reduction(482)] or through collision with free thiols such as glutathione. There is also evidence that reduction of some enzyme disulfides by free GSH may be considerably faster than the reduction of generic disulfides, while free GSH-mediated reduction of other enzyme disulfides is much slower than expected.(483) Experimentally, protein sulfenylation has been reported to peak 5 min post EGF stimulation followed by a decay over 30-60 minutes.(484) This relaxation time implies oxidation is faster than re-reduction and that "sulfenylated protein" has a global half-live on the order of 5-10 min in cells. Several factors could contribute to this result: the oxidized sulfurs may be privileged, and the sulfenic acid products could be stabilized or sterically shielded from reductants. Recently it has also been reported that some sulfenic acids can form sulfenamides (adduction to an amine) or the analogous sulfenylamides (adduction to the amino function within an amide). These structures may be more stable to reduction than sulfenic acids, potentially offering a means to stabilize oxidized proteins and prolong signals or regulate re-reduction more tightly.

Reduction of sulfenic acids or disulfides can generate secondary disulfides and beyond. This opportunity enables redox signaling cascades to function through "changing of hands", after the initial redox-signal modification of the first responder. In this scenario, an energy-neutral process (such as disulfide exchange) transfers the redox signal from one protein to another, while simultaneously transferring information. 
Further oxidation of the sulfenic acid is not as easy as the first oxidation step, at least for electrophilic oxidants. Reduction of sulfinic acid is only achieved by a few enzymes and so the reversibility of this modification is context dependent. Sulfiredoxin is an ATP dependent enzyme that reduces sulfinic acids of 2-cysteine peroxiredoxins to the corresponding sulfenic acids. This enzyme appears to be the principal means to reduce peroxiredoxin sulfinic acids in A549 cells.(485) Interestingly, compared to the prokaryotic counterpart, eukaryotic 2-cysteine Prx are almost 100-fold more susceptible to overoxidation. This has led to the 'floodgate model' for 2-cysteine Prx regulation.(486) In this model, eukaryotic 2cysteine Prx acts as a "redox buffer" preventing peroxide-mediated oxidation of other redoxsensitive proteins until the floodgate is opened by overoxidation of the Prx enzyme. Sulfiredoxin, on the other hand, is a relatively slow enzyme $\left(\mathrm{k}_{\mathrm{cat}}=0.003 \mathrm{~s}^{-1}\right)$, yet it shows significant resistance to oxidation.(485) This protein also removes other oxidative modifications from peroxiredoxin in an ATP-dependent manner.(487) Sulfonic acid formation is very slow, and there are no known cellular reduction mechanisms for this product. This signal is also a degron in some cases,(488) rendering reversibility a moot point.(489)

\subsubsection{Carbon oxidation is irreversible, but requires high-energy oxidants and can proceed with unusual regioselection-High-reactivity ROS (e.g., $\left.{ }^{\circ} \mathrm{OH}\right)$ target} labile $\mathrm{C}-\mathrm{H}$ and $\mathrm{O} / \mathrm{N}-\mathrm{H}$ bonds. These reactions are irreversible. The reaction of ${ }^{\circ} \mathrm{OH}$ with $\mathrm{C}-$ $\mathrm{H}$ has a rate constant of $10^{8} \mathrm{M}^{-1} \mathrm{~s}^{-1}$.(490) There is known to be some residue and protein dependence to this rate constant(491) and indeed hydrogen abstraction second-order rate constants from amino acids range from $10^{7}$ (Gly) to $10^{10}$ (Trp, His) $\mathrm{M}^{-1} \mathrm{~s}^{-1}$.(490) This is unsurprising since radical reactions are strongly dominated by stabilities of product radicals and bond dissociation enthalpies, and so attack at weak bonds to give more stable radicals will always be favored. Dative stabilization confers significant longevity to radicals so abstraction of hydrogen atoms a-to heteroatoms where a lone pair is free to stabilize the product radical is one of the most common mechanisms. This process is further favored because ${ }^{\circ} \mathrm{OH}$, and similar biological radicals (such as ${ }^{\circ} \mathrm{OR}$ ), are electrophilic by nature, so favor formation of a positive charge on the carbon bearing the abstracted hydrogen in the transition state.(492) Indeed, glutamic semi-aldehyde, the product of hydrogen abstraction from arginine, is a common process,(493) although products that proceed through formation of secondary aliphatic radicals (e.g., hydroxyglutamine) have been reported.(494) By extension, serine and threonine are also susceptible to hydrogen abstraction.

There is also evidence that polar residues surrounding the site of hydrogen abstraction increases the rate of oxidation.(495) Capto-stabilization is also a significant stabilizing element for radicals and so $\mathrm{H}$-abstraction a-to side chain carboxylic acids and amides is also facile(491) (although this is less favored on charge distribution grounds than abstraction a-to a donating group). Finally, captodative stabilization affords the most stabilized radical,(496) and thus it may be expected that abstraction at the a-hydrogen would dominate radical reactions with peptides. Unlike other sites of radical abstraction, reaction at the a-position leads to fragmentation of the polypeptide backbone and this is likely significantly damaging to the protein and by extension the cell.(497) Although such a-hydrogen abstractions do occur, peptides are on the whole refractory to this process [abstraction at non-captodative 
stabilized sites occurs 10-1000 times faster(498)]. This unexpected regioselection may explain the stability of peptides in oxidizing environments.(499) This problem has been addressed computationally by several authors, with hydrogen bonding being suggested as a means to suppress attack at the a-position.(500) Regardless of the position of abstraction, the carbon-based radical product of hydrogen abstraction by ${ }^{\circ} \mathrm{OH}$ is typically trapped by ${ }^{3} \mathrm{O}_{2}$ (likely a diffusion controlled process in water). The resulting organic radical peroxide can liberate a molecule of ${ }^{1} \mathrm{O}_{2}$ during hydrolysis. As discussed above, ${ }^{\bullet} \mathrm{OH}$ reactions can thus constitute a bifurcating signal exchange and consequentially in the vicinity of ${ }^{\bullet} \mathrm{OH}$, there will be a local increase of several ROS species, including ${ }^{1} \mathrm{O}_{2}$ (and oxidized proteins).

\subsubsection{RES modifications can be reversible or irreversible-Many RES}

modifications are irreversible, rendering changing of hands unlikely. Thus, most RESmodified proteins must be privileged in order to function as sensors. Although there are some counter-examples,(501-503) HNE and similar protein-enone adducts have been resolved by SDS PAGE and detected by mass spectrometry indicating that many HNE adducts are at least reasonably stable. Nitroolefins on the other hand can form reversible adducts to cysteines(504)(Figure 9). The dissociation constant of nitro-oleic acid from cysteine thiolate is around $10 \mu \mathrm{M} .(92,505)$ Interestingly, the apparent second order adduction rate constant (which is $\mathrm{pH}$ dependent) is $355 \mathrm{M}^{-1} \mathrm{~s}^{-1}$ (at $\mathrm{pH}$ 7.4).(95) Accordingly, nitroolefins likely have very different signaling properties to most other endogenous RES. The difference in adduct stabilities between enals and nitroolefins may be due to: (1) the 13 unit difference between the $\mathrm{p} K_{\mathrm{a}}$ of the protons a-to the carbonyl [30; methyl acetate(506)] and nitro groups [17; nitromethane(507)]. In fact, several drug enone-derived discovery efforts have found increased $\mathrm{p} K_{\mathrm{a}}$ of protons a-to the enone (or similar mesomerically stabilizing group) leads to better reversibility;(508) and (2) the ability of enal-adducts to undergo further reaction post cysteine conjugation (a process not possible for adducts to nitroolefins; Figure 9).(509) Furthermore, although it is unclear whether this process occurs in cells or during handling post-lysis, we and others have characterized the aldehydereduced version of cysteine-Michael adduct of HNE conjugated to Keap1 enriched from cells, following bulk HNE challenge of live cells or controlled targeted delivery of HNE in situ in live cells. This product is unable to enolize, and hence is irreversible. It is likely that this unexpected reduction process contributes to enal adduct stabilities. Reversibility can also be dependent upon which functional group is modified. For instance, 2-pentylpyrrole lysine is believed to be an end product of lysine adduction to HNE.(510) Formation of this product is likely irreversible.

Cysteine also forms adducts to isothiocyanates (ITCs). These cysteine adducts are in principal freely reversible and can show varying stability. In some instances, the ITC can migrate from the initial point of contact at cysteine to adjacent amine adducts forming thioureas that are essentially irreversible. Critically, such transfer is possible for $\mathrm{sp}^{2}$ hybridized carbon adducts that occur through addition/elimination. On the other hand, direct transfer of RES is not possible for $\mathrm{sp}^{3}$-hybridized adducts, like those formed to nitroolefins and enones because this process must proceed through direct $\mathrm{S}_{\mathrm{N}} 2$ at the carbon sulfur bond (Figure S4). Thus, for RES, the possibility of change of hands (i.e., direct transfer of chemical information from one protein to another) is dependent on hybridization of the 
carbon to which the cysteine adduct occurs. It should be noted that most RES adducts are in principle reversible, even if the reverse reaction is often very slow or requires harsh conditions.

It is believed that for HNE, adducts on residues other than cysteine dominate thermodynamically. For instance, histidine adducts to HNE may be more stable than cysteine adducts and as we pointed out above, this difference in stability may explain the preponderance of amine-HNE adducts identified especially in the early literature.(513) However, it is likely that cysteine is always the first residue modified due to its better efficiency in Michael addition. In practice translocation to histidine may not occur at a rate that competes with other processes such as degradation or signal transduction for many adducts, although this has not been systematically studied.

\subsection{Implications for tracking signaling pathways}

Irreversible modifications make tracking redox signaling relatively simple: assuming ideal detection methods and little decay/transfer, the whole gamut of proteins modified irreversibly can be obtained through MS analysis or profiling. Unfortunately, detection methods are not ideal (see section 5.2) and covalent bond formation is not mandatory for biological activity so one cannot assume that profiling/enrichment analysis is all encompassing. However, the irreversibility of modifications like HNEylation has been used to the advantage of many researchers trying to establish protein targets. On the other hand, it can be quite difficult to trace the "initial point of origin" for reversible/exchangeable modifications.

\subsubsection{Implications for regulation of RES/ROS signaling-Signaling pathways} likely need spatial/temporal and chemical regulation to ensure high fidelity of information transfer. A simple discussion of diffusion distance and reactivity as presented in the opening paragraphs of this section (section 3.1) makes a case that "sphere of influence" (localization due to inherent practical limits to diffusion distance) and chemical constraints (kinetic privilege) can orchestrate selective redox signaling in cells.(514) This argument is bolstered when it is considered that macromolecules are essentially stationary amongst the hustle and bustle of small molecule diffusion in the cell. Thus, a local burst of reactive molecule in proximity to a privileged (immobile) sensor protein can lead to high local concentrations of a "modified" protein state, even if the bulk protein (that exists elsewhere in the cell) is unmodified.(515) But, given the crowded environment of the cell, the huge number of adventitious thiols and the efficiency of detoxifying processes, it is likely that intended signaling targets have attributes of privileged sensors. For such a mechanism to function adequately, sensor proteins either have to be enriched in a particular area of the cell primed for reaction, or else ubiquitously expressed. If the signal is released but the protein is not present to accept it, there is likely not sufficient time for the sensor to arrive before the signal is lost or misdirected, meaning the required response is not triggered and potentially causing damage by modifying the wrong protein.

Even assuming that a sensor is present, the chances of modifying the incorrect protein increase if intrinsically hyper-reactive molecules such as ${ }^{\circ} \mathrm{OH}$ carry the signal. For reagents 
that react at the diffusion limit even in the absence of catalysis, conferring enzyme-like sensing capacity to a sensor would unlikely confer a huge amount of selection. For this reason, privileged ${ }^{\bullet} \mathrm{OH}$ sensors are unlikely attainable and selective signaling modes likely occur purely through proximity enhancement if indeed molecules like ${ }^{\circ} \mathrm{OH}$ do behave as signals at all. It is thus likely privileged ROS/RES sensors only act on relatively lowreactivity signals, where kinetics and regioselection can be carefully controlled through selective rate amplification.

\subsection{Unusual conditions and chronic or excessive exposure to ROS/RES may lead to modification of residues other than cysteine}

\subsubsection{Reaction of residues other than cysteine with ROS}

3.11.1.1 Reaction of methionine with ROS: Methionine can be oxidized relatively efficiently.(516) Unlike cysteine and acetyl cysteine, the oxidation of methionine (although slow) by peroxide is $\mathrm{pH}$ independent $\left(10^{-2} \mathrm{M}^{-1} \mathrm{~s}^{-1}\right)$ and can in fact dominate glutathione and acetyl cysteine oxidation (by $>10$ fold) below $\mathrm{pH} 4$. However, by $\mathrm{pH} 6$, glutathione is $4-$ 5 fold faster in terms of peroxide reaction than methionine, likely due to partial formation of the GSH thiolate. This indicates that in acidic organelles, methionine could be the most relevant signaling target, if signaling occurs in such regions. Interestingly, peroxymonocarbonate [formed from reaction of bicarbonate with peroxide, a process that occurs with a rate constant of $\left.10^{-2} \mathrm{M}^{-1} \mathrm{~s}^{-1}(517)\right]$ oxidizes methionine much faster than peroxide oxidizes methionine $\left(0.5 \mathrm{M}^{-1} \mathrm{~s}^{-1}\right)$.(518) Of course, peroxymonocarbonate is a better oxidant of cysteine, although the rate acceleration may be less significant than for methionine (around about 20-fold for protein tyrosine phosphatase and only two-fold for papain).(519) Reduction of sulfoxides is much more difficult than sulfenic acids; for instance, the second-order rate constant for the reaction of glutathione with DMSO is $0.00005 \mathrm{M}^{-1} \mathrm{~s}^{-1}$.(520) Thus methionine-S-oxide requires enzymatic reduction. Methionine $S$-oxide is chiral due to the increasing difference in s-p orbital energies as one proceeds down a group of the periodic table,(521) shutting down of the "umbrella inversion" effect in Period-3 elements. Reduction of the $R$ and $S$ forms of methionine- $S$-oxide is carried out by two separate enzymes.(522) Given the differences in rates of chemical oxidation of cysteine and methionine as well as the shorter half-lives of sulfenic acids relative to methionine- $S$ oxides, it is very likely that these two modifications fulfill very different roles in cells. As was noted above, $\mathrm{HOCl}$ can oxidize methionine with similar efficiency to cysteine. It is believed that $\mathrm{HOCl}$ generated from myeloperoxidase may be involved in modification of several proteins,(523) including inhibition of cholesterol export by ABCA1.(524)

Methionine oxidation can also occur through enzyme catalysis. The NADPH/flavin dependent monooxygenase MICAL is the best example.(525)

3.11.1.2 Reaction of histidine with ROS: Oxo-histidine on proteins has also been observed. This is most commonly formed from treatment of proteins with copper $/{ }^{3} \mathrm{O}_{2} /$ ascorbate. Growing evidence indicates that this may be a signaling residue. For instance, Bacillus subtilis PerR forms oxo-histidine in response to reaction with $\mathrm{H}_{2} \mathrm{O}_{2}$.(526) This modification decreases PerR's ability to bind DNA. Other regulatory roles of oxo-histidine have been proposed, but these likely proceed through enzyme-catalyzed processes.(527) 
3.11.2 Reaction of residues other than cysteine with RES-As discussed above, enzymes can confer unusual properties to amino acid residues. Depression in $\mathrm{p} K_{\mathrm{a}}$ (and other factors, such as those described above) can endow the residues of interest with higher-thanexpected nucleophilicity. In the case of lysine, deprotonation is required for nucleophilic addition, whereas histidine is only partially protonated at $\mathrm{pH} 7$, so the nucleophilicallyactive form is accessible. For instance, some electrophilic suicide inhibitors, like vigabatrin, target active site lysines that function as catalytic bases in the target enzyme. Inactivation of glucose-6-phsophate dehydrogenase by HNE in vitro has been proposed to proceed by adduction to lysine. It is noteworthy that the $\mathrm{pH}$ rate profile for this lysine adduction process showed that the target lysine had a $\mathrm{p} K_{\mathrm{a}}$ of 10 , indicating that it may even be deactivated(528) relative to the "free" lysine. Lysine-rich enzymes, such as ubiquitin form HNE adducts in vitro.(529)

In similar experiments, GAPDH was shown to form adducts to HNE via cysteine, lysine, and histidine with the distribution in part being dependent upon presence of a substrate (NAD),(530) indicating that at "physiological conditions" ligands can influence the site and residues modified by RES. It should, however, be noted that these experiments were conducted with millimolar HNE. Subsequent experiments showed that for GAPDH, adduction at cysteine and histidine are more rapid than lysine.(531) Interestingly, the same paper showed that the active-site cysteine was not modified by HNE, with surface residues being preferred. HNE adducts to both cysteine and histidine are implicated in the inhibition of cathepsin B.(532) Enzymes that metabolize HNE may show some robustness to HNE modification. For instance, GST-A4-4 (a protein principally responsible for HNE metabolism through glutathione conjugation in the liver)(533) is significantly less susceptible to HNE adduction and inhibition than other GST isoforms.(534)

One important modification is 4-ONE-ylation of lysine. This modification occurs through amine adduction to the aldehyde function to form a hemiaminal. This function then undergoes tautomerization to form the $\gamma$-keto amide.(535) Intriguingly there is evidence that SIRT2, a histone deacetylase, can remove this modification, at albeit at a rather sluggish rate $\left(500 \mathrm{M}^{-1} \mathrm{~s}^{-1}\right)$.(536) Thus, this is possibly an example of a removable (not strictly reversible) protein RES modification.

Although interesting, the above experiments have not commuted well to real world situations. Bolus treatment of cells with HNE showed that cysteine adducts dominated over histidine adducts by around 40:1.(537) Furthermore, no HNE-lysine adducts were observed in lens protein extracts from patients aged 1-74 years old,(538) indicating that even for longlived proteins (crystalline, a major component of the eye lens that is not degraded through life) HNE lysine adduction is slow. However, increase in histidine bound to HNE metabolites in urine has been proposed as a biomarker for carbonyl stress.(539) Histidinyl HNE is also elevated in patients with Alzheimer's disease.(540) Thus, although histidine adducts to HNE are uncommon in healthy cells, these adducts may accumulate in (some) diseased states. Interestingly 1,4-quinones are reportedly targets of lysine-rich motifs (541) although they can also be attacked by glutathione. Reoxidation of adduction products can allow multiple adduction events to take place.(542) The reaction of 1,2-quinones with amine functions is $10^{5}$-fold slower than adduction to thiols.(543) 
Selenocysteine [Sec, $\mathrm{p} K_{\mathrm{a}}$ 5.2(544)] is a genetically encoded analog of cysteine that is present in around 25 proteins in humans.(545) Although the precise reasons for incorporation of Sec into proteins is debated, $(546,547)$ it is clear that Sec is intrinsically more nucleophilic than cysteine.(548) Unsurprisingly, Sec is a target of both ROS and RES. Glutathione peroxidase, a selenoprotein, was shown to be oxidized at selenium to form a selenylamide that can be reactivated by addition of glutathione.(549) The selenoprotein, thioredoxin reductase, was shown to be inhibited by $\mathrm{HNE}\left(\mathrm{IC}_{50}=4 \mu \mathrm{M}, 2 \mathrm{~h}\right.$ treatment). $\mathrm{HNE}$ adduction was observed at C496/Sec-497, which are located in the active site. Acrolein also alkylates thioredoxin reductase at Sec.(550) The half-life of this adduct was 4-8 h, and reversal required both GSH and thioredoxin 1.(551) Given that selenocysteine-carbon bonds are weaker than carbon-sulfur bonds, the carbon selenium bond is likely a better acceptor of electrons through negative hyperconjugation,(552) and that $\mathrm{SeH}$ is more acidic than $\mathrm{SH}$, (553) it is likely that Michael adducts to protein-selenocysteine are shorter lived than protein-cysteine, assuming there are no cross links/additional protein interactions.

3.11.3 Reaction with other macromolecules-The tacit assumption of this section has so far been that protein is the sole macromolecular target of ROS and RES (although these are formed by action of RES on lipids). The assumption that proteins are the principal target is mostly justified because proteins are both abundant and rich in deprotonatable $\mathrm{sp}^{3}$ hybridized sulfur. The RNA:protein mass ratio in cells ranges from 0.1-0.5.(554) DNA is less abundant than RNA.(555) Furthermore, although RNA can contain thiol groups,(556) these are $\mathrm{sp}^{2}$-hybridized and hence have low nucleophilicity/acidity compared to thiol/ thiolate pairs. Thus, by both mass action and second-order rate constants, oxidation and electrophilic adduction to nucleic acid through nucleophilic attack is likely to be much slower than to protein.

Cofactors/prosthetic groups can also be the target of ROS. Iron-sulfur proteins are among the most ROS-sensitive architectures in the cell.(557) For instance, PP1 is inhibited by peroxide $\left(\mathrm{IC}_{50}=70 \mu \mathrm{M}\right)$, via attack on the catalytically-essential $\mathrm{Mn} / \mathrm{Fe}$ (II) prosthetic group. In addition, some iron-sulfur clusters sense ROS and coordinate responses to changes in ${ }^{3} \mathrm{O}_{2}$.(558) Other essential $\mathrm{Fe}-\mathrm{S}$ proteins, such as Rli1p(ABCE1) are highly susceptible to ROS.(559) Furthermore, ROS functions synergistically with cofactors. A mixture of acetoacetyl-CoA and $\mathrm{H}_{2} \mathrm{O}_{2}$ is able to initiate oxidation of NADH.(560)

Nucleic acids are sensitive to attack by radical-based ROS. ${ }^{\circ} \mathrm{OH}$ attacks DNA in a diffusionlimited process.(561) Indeed, the mechanism of action of the DNA-damage inducer, bleomycin, is believed to proceed by oxidative modification of DNA(562) and more than 20 separate types of nucleic acid damage have been reported to be caused by ${ }^{\circ} \mathrm{OH}$ alone. RNA is the most ROS-susceptible nucleic acid in the cell.(563) HNE [and other endogenous electrophiles(564)] can modify DNA, principally through adduction to deoxyguanosine. (565) This adduct can be repaired (probably by nucleotide excision repair), however the HNE adduct is estimated to be more mutagenic than 8-oxoguanosine,(566) possibly due to formation of crosslinks.(567) HNE-DNA adduction is elevated 4-fold relative to controls upon glutathione depletion in rats(568) and elevated HNE-DNA adducts have been observed in human patients with nonalcoholic fatty liver disease. $(569,570)$ The most reactive of the common LDEs, ONE,(571) reacts with tRNA in rat intestinal epithelial cells,(572) in 
addition to other nucleic acids.(573) HNE may also react with aminophospholipids.(248) This process (in addition to adduction to protein thiols) affects the activity of adenine nucleotide exchange factor in liposomes.(574)

\subsection{What makes a privileged sensor?}

Combining the above discussions, the definition of privileged sensor is currently functional (beyond initial considerations in section 3.10.1): a protein that is able to engage with (a redox) signal preferentially over other species in the cellular milieu leading to a downstream signaling response. It is unlikely that redox signaling can ever be $100 \%$ intended protein selective, so privileged sensors likely have mechanisms to promote "on-target" downstream signaling. These mechanisms may involve (1) increased reactivity to the signal in question; (2) proximity of release and target protein; and (3) coupling redox-signal modification event to a phenotypically-dominant effect (such as gain of function or dominant loss of function). It is often assumed that enhancement arises from increased thermodynamic acidity of privileged sensor. However, to be privileged, additional factors that give an extra boost to reactivity are required. Pre-binding of the electrophile prior to covalent bond formation may increase reactivity for HNE. Interestingly, glutathione reductase is inhibited by HNE but is refractory to inhibition by 2-oxoaldehydes,(575) suggesting that intrinsic reactivity of the electrophile is not a key determinant in this process. This example highlights that one should not assume just because a molecule is more electrophilic it will by default react faster with a specific protein thiol. Abundant examples of such selectivity exist in the literature, including the reactivity of linear enals with tubulin.(576) Similar to Pfeifer's rule in medicinal chemistry that defines a positive correlation between enantioselectivity and inhibitor potency,(577) as the protein thiol becomes more matched with a specific electrophile, it is likely that its reactivity with other electrophiles, even those with similar structure, will decrease. Interestingly, different enantiomers of HNE elicit widely different phenotypic effects in mouse hepatocytes.(578) This observation could be due to differential metabolic/ excretion processes and/or different target profiles. However, certain enzymes are known to react preferentially with specific enantiomers of HNE. One of the best examples is human thioredoxin that contains two HNE-reactive thiols. The more exposed reactive cysteine (C73) does not react enantiospecifically, whereas the more buried reactive cysteine (C32) shows 3:1 preference for $(R)$-HNE.(579)

\subsection{1 $\mathrm{p} K_{\mathrm{a}}$ is believed to determine kinetic modification site, but the situation} is complex-Thiolate is believed to be the active nucleophile in most addition or direct thiol oxidation reactions. Cysteine has a $\mathrm{p} K_{\mathrm{a}}$ of 8.5 in water. Thus, in the cellular milieu, cysteine is mostly in its neutral form and not ideal for reactivity. Although some theoretical work indicates that the difference in nucleophilicity between the anion and conjugate acid can be decreased by solvent effects in enzyme active sites, most nucleophilic thiols have anomalously low $\mathrm{p} K_{\mathrm{a}}$ values.(580) However, relative to the equilibrated thiol/conjugate base mixture at $\mathrm{pH} 7.4$, complete deprotonation of DTT, GSH and N-acetyl cysteine can only maximally confer 20-150-fold increase in reaction rates. For simple enones (acrolein and methylvinyl ketone) reacting with cysteine, the rate enhancements are no more than 10-fold upon full deprotonation.(581) For peroxide reaction with thiols, it has been concluded (albeit by comparing thiols with relatively small $\mathrm{p} K_{\mathrm{a}}$ differences, $<2$ units) that a maximum rate 
constant of around $20 \mathrm{M}^{-1} \mathrm{~s}^{-1}$ is achievable through $\mathrm{p} K_{\mathrm{a}}$ perturbation (a 20 -fold increase in reactivity relative to the thiol/thiolate at neutral $\mathrm{pH}$ ).(431) Furthermore, for reactions involving disulfide bond cleavage by thiol nucleophiles, thiolate nucleophiles formed from thiols with high $\mathrm{p} K_{\mathrm{a}}$ are more nucleophilic than thiolates formed from low $\mathrm{p} K_{\mathrm{a}}$ (by approximately 10 -fold over a 3 -unit $\mathrm{p} K_{\mathrm{a}}$ range). Thus, lowering $\mathrm{p} K_{\mathrm{a}}$ of a thiol alone (i.e., stabilizing the anionic form) may countermand nucleophilicity of the thiolate in the absence of other factors.(483)

This effect can be readily rationalized by considering HOMO-LUMO overlap. As the $\mathrm{p} K_{\mathrm{a}}$ of the thiol decreases, the HOMO of the thiolate anion lowers, leading to decreased overlap between the nucleophile and the electrophile LUMO. A linear free energy relationship between $\log$ (rate thiolate oxidation) and $\mathrm{p} K_{\mathrm{a}}$ (thiol) was maintained across $4 \mathrm{p} K_{\mathrm{a}}$ units. The gradient $(\beta)$ of this relationship, a measure of charge loss in the transition state, was 0.4. This result indicates that raising of the energy of the thiolate (higher $\mathrm{p} K_{\mathrm{a}}$ ) quite considerably promotes reaction. Many peroxidases and other peroxide reacting protein bucked this trend, i.e., maintained high reactivity even though the $\mathrm{p} K_{\mathrm{a}}$ of the anion was low. This gives strong evidence that "reaction privilege" is a function of numerous factors and that they must act to maintain thiolate reactivity.(409)

To achieve enzyme-like rates (for instance, peroxiredoxin has $k_{\text {cat }} / K_{\mathrm{m}}>10^{6} \mathrm{M}^{-1} \mathrm{~s}^{-1}$ for $\left.\mathrm{H}_{2} \mathrm{O}_{2}\right)(107,111)$ other factors than thiolate formation are certainly at play.(582-584) A similar conclusion can also be derived from considering GST.(585,586) Ground state destabilization by desolvation, a common "trick" employed by enzymes to raise the HOMO and hence increase rates that provides many orders of magnitude increase over second order uncatalyzed rates.(587) Interestingly, the rates of reaction of thiol with peroxide and hypochlorite differ by $10^{7}$-fold. This comparison clearly shows that leaving group potential can have a much larger impact on reactivity than thiol deprotonation.(116) Additionally, transition-state stabilization(588)and binding prequilibria may also be used. These aspects are believed to be used by peroxiredoxin to enhance reaction rates.(588)

3.12.2 Structural motifs-Since the above discussion implies that structure will determine redox-sensitivity of proteins, there has been considerable effort invested into understanding what makes a specific cysteine kinetically privileged. Although it is not the only important aspect, these studies have largely centered on thiolates within enzymes (i.e., active-site, catalytically-essential cysteines) and as such may or may not be applicable to all privileged sensors. For instance, three key parameters have been suggested to confer high propensity to form reversible (signaling) disulfides in proteins: distance to nearest cysteine sulfur, solvent accessibility, and $\mathrm{p} K_{\mathrm{a}}$.(589) Intriguingly, proximity to cysteine appears to be a reasonably important parameter promoting propensity for oxidation, although the reasons for this are unclear.(590) As we have seen, $\mathrm{p} K_{\mathrm{a}}$ modulation can confer a reasonable win in terms of reactivity; thus, it makes sense that this calculable factor should be considered first. Many arguments appear to use variants of the Westheimer hypothesis, $(591,592)$ i.e., a chargecentric view: positive charges adjacent to reactive cysteines will stabilize the thiolate and promote nucleophilicity.(593) For instance, cysteine proteases contain a catalytic dyad consisting of a reactive cysteine within hydrogen bonding distance of an imidazole ring. Since proton transfer from the thiol to the imidazole ring is likely significant, this can be 
considered as a case of proximal charge-charge stabilization. Model cysteine-containing peptides are 5-times more nucleophilic to HNE if they contain an arginine.(450) Studies with tubulin concluded that proximal positive charges promote cysteine alkylation, whereas negative charges suppress alkylation;(594) adjacent positive charges also seem to promote thiol exchange in several proteins (by around 2 orders of magnitude).(595,596) Both the effects on alkylation and disulfide exchange have been ascribed to $\mathrm{p} K_{\mathrm{a}}$ changes. Model thiol exchange kinetics measurements back up that rate enhancements on the order observed may be possible. On the other side of the spectrum, proximal glutamic acids endow a specific cysteine with low $\mathrm{p} K_{\mathrm{a}}$ in human DJ-1.(597) Proximity to a helix dipole can also stabilize thiolate anions,(598) for instance in E. coli thioredoxin.(599)

However, this view has not always held up across multiple enzymes. For instance, a lysine proximal to $\mathrm{C} 22$ of human Grx 1 has little effect on $\mathrm{p} K_{\mathrm{a}}$.(600) Proximal negative charges also exert little effect on cysteine $\mathrm{p} K_{\mathrm{a}}$ in DsbA.(601) Indeed, hydrogen bonding may be the most decisive factor in determining thiol $\mathrm{p} K_{\mathrm{a}}$ at least in some enzyme active sites, such as yeast DJ-1 (602) and mouse methionine sulfoxide reductase A. (603) Interestingly, it is quite well established that cysteine is much more particular about its hydrogen bond donor partners than its acceptor partners. However, how this may affect reactivity is unknown, although it does mean that a general base can activate cysteine from many more positions than a general acid can stabilize a thiolate.(604) More information especially pertaining to privileged sensors and what rate enhancements they can obtain is required to be able to understand the intricacies of what makes a sensor. Nevertheless, since 500-fold rate enhancements (like those above) have been reported, it is likely that $\mathrm{p} K_{\mathrm{a}}$ alone is not the only (or likely not the operative) factor.

3.12.3 Implications for specificity-It stands to reason that if privileged sensors do not just accelerate reaction by deprotonation of sensor cysteines, then the sensor protein may recognize specific aspects of the reactive signal. In other words, the sensor protein may be tuned to react with specific ROS or RES. As we mentioned above, as a sensor is tuned to a specific signal, there will be more discrimination between different electrophiles/oxidants. None-the-less, many enzymes can achieve sizeable rate enhancements while maintaining moderate-to-high promiscuity. These enzymes include GST-A1-1 that metabolizes androstenedione, HNE, hydroxydecenal, chloro-dinitrobenzene and epoxy pyrenes with $k_{\text {cat }} / K_{\mathrm{m}}$ around $10^{5} \mathrm{M}^{-1} \mathrm{~s}^{-1}$.(605) Interestingly, the chemical reaction catalyzed by GST is not dissimilar to the sensing function carried out by many privileged RES sensors. $(605,606)$ Several lines of reasoning are consistent with a picture in which privileged sensors interact with their substrates/stabilize transition states as well as promote bond formation. (1) We have uncovered isoform-divergent electrophile/oxidant sensors, indicating that some aspect of specific isoforms renders ROS- or RES-specific sensitivity/responsivity.(512) (2) Keap1 is a multipurpose ROS/RES sensor is equipped with 27 cysteines many of which have been implicated in redox sensing/signaling. $(607,608)$ Comparison of delivery of HNE and various other electrophiles to Keap1 shows that Keap1 can sense both cyclic and linear electrophiles similarly.(609) However, we have shown that under electrophile-limited conditions wherein RES signals are delivered under controlled conditions in situ, different residues are targeted for cyclic RES (C613)(389) and for acyclic RES (C513/C518).(259) 
3.12.4 Model systems-Decoding evolutionary information from model redox sensors is difficult: cysteine is in general a conserved amino acid. It has further been found that conservation of cysteine is favored by clustering and when it is buried within the protein structure.(367) These "character traits" make conservational analysis of putative redox sensors (typically surface exposed and isolated) fairer and less likely to be influenced by the inherent conservation preference of cysteine. We can thus cautiously use phylogeny to analyze privileged sensors. We have identified Akt3 as a sensor of RES. Another laboratory has independently identified Akt2 as a ROS sensor. Interestingly, these two enzymes share different local topographies around their specific sensor cysteines. Since Akt2 and Akt3 are highly homologous except in the linker region where the sensor cysteines lie, this may suggest that ROS and RES sensing are at least to some level favored by different local environments around the sensor cysteine. For instance, Akt3 has two anion-rich sites and Akt2 has none. Consistent with the linker sequence being important for sensing, for a specific isoenzyme, there is little divergence in the linker region across mammals, reptiles, fish and birds and the anion sites are fully retained (Figure 10). Interestingly, Akt2 has the ability to sense RES weakly, although this sensing appears to not be coupled to function. Obviously, it is possible that Akt2 cannot be labeled sufficiently to elicit a phenotypic downstream output. However, it is also possible that RES sensing by Akt2 is unable to drive phenotypically-dominant loss of function effects that are manifested in Akt3 RES-sensing. We are now investigating these mechanisms to begin to understand correlations between dominant responses and increased cysteine nucleophilicity. It is likely that the Akt $2 / 3$ system will serve as a paradigm for ROS/RES sensing in the future and may hold some answers to unresolved questions.

The field, especially for ROS signaling, seems to have reached a consensus that specific protein redox modifications are important. However, disagreement remains over how cells achieve specificity through redox signaling. This is unsurprising as it is a very complex problem and, biology is pragmatic at finding solutions to problems and so it is unlikely one single solution will apply to all scenarios. Thus, the current debates about local ROS elevation versus channeling versus privileged sensing - for instance, primary sensing by peroxiredoxins vs. other direct/indirect ROS-sensors-are similar to classic debates in science about diversity in the immune response and factors leading to enzymatic rate accelerations. We hope that in the future years there will be a compromise allowing us to parse these variables, clearly define terms, and understand signaling contributions for specific proteins and specific ROS/RES.

\section{MECHANISMS OF RES/ROS SIGNALING}

Here we discuss specific ways in which initial RES/ROS insults cause signaling using specific examples. We stress that we have only scratched the surface of what constitutes a physiologically-relevant redox sensor and so the examples currently available may not be generalizable or even describe the best sensors. For instance, many "protein redox sensors" studied using isolated proteins in vitro have slow second-order rate constants of association with their putative signals. Unsurprisingly, in vitro experiments-especially in the study of non-enzyme-assisted RES/ROS modifications-do not transpose well to conditions in cells or whole organisms. Several reasonable explanations have been postulated to reconcile what 
is seemingly a dichotomy between in vitro and cell-based data, including: (1) high localized concentration/compartmentalization of small molecules in vivo may circumvent low secondorder rates of reaction for specific proteins, forcing a reaction to occur by mass action; (2) compartmentalized changes in environment (e.g., $\mathrm{pH} /$ associations) may also promote/assist protein modification; (3) there may be competition with other thiols within specific cellular microenvironments (small-molecule such as GSH or proteins); (4) locale/context-specific (and likely dynamic) protein redox states-that can alter protein conformation, specific cysteine reactivity, etc.-is not easily recapitulated in vitro. However, many reinforcing factors are required to convert intrinsically low second-order reaction rates $\left(1-100 \mathrm{M}^{-1} \mathrm{~s}^{-1}\right)$ and requirements of unusually high $\mathrm{pH}$ to observe a reaction in vitro, into a physiologicallyrelevant signaling axis. Thus, it is our opinion that much of the current experimental data do not instill the highest confidence that the observed modifications are relevant.(509) After all, free amino acids perform chemistry associated with many of the proposed signaling functions similarly to proposed sensors, meaning mass action alone is unlikely to lead to (semi-) selective labeling.

Hopefully in the future more in-cell relevant sensors will be discovered that can sense redox signals in unstressed cells with low levels of signal used, ideally with endogenous levels (or below) of the sensor. Nevertheless, redox signaling by both RES and ROS are now clearly implicated in a wealth of different specific signaling processes in cells including AR, growth signaling, apoptosis, and DNA damage. We discuss methods and toolsets to try to assist in the quest for bona fide sensors later in section 5. In this section, we critically review some of the data linking redox signaling to specific modifications and models as to how those modifications are maintained in cells.

\subsection{Oxidation of cysteines to $-\mathrm{SOH}$}

Sulfenic acid is the foundational, common chemical step in production of disulfides, sulfinic acids, and the panoply of other oxidized cysteine modifications. These species exist as an equilibrium of sulfenyl and sulfinyl tautomers,(610) although the sulfenyl form is most commonly drawn and is likely the more stable. As discussed in section 3, sulfenic acid can be created from interaction of thiols with a host of ROS (Figure 11)(611) and nucleophilic attack by hydroxide $(612,613)$ or a carboxylate on a disulfide. The latter proceeds via a mixed sulfenic/carboxylic acid anhydride(614) that is likely not favored in the absence of an enzyme active site.(615) Sulfenic acid may also arise from hydrolysis of nitrosocysteine. (616) In some special cases, sulfenyl iodide has also been isolated,(617) although the relevance of this functional group in vivo is unknown. Sulfenic iodide appears to be readily and reversibly displaced by chloride ions and can also be hydrolyzed under the correct conditions.(618)

\subsubsection{Several enzymes have evolved to form stable sulfenic acids that resist} further oxidation-As we discussed above, most sulfenic acids are short lived, due to kinetically fast reaction with other thiols to form disulfides. However, some proteins are susceptible to sulfenic acid formation. One key class of proteins susceptible to sulfenic acid formation is cysteine proteases. Some of the key cysteine proteases in eukaryotic cells include deubiquitinating (DUBS) enzymes, deSUMOylating enzymes, and cathepsins. Many 
of the 5 classes of thiol protease DUBS are redox active. $(619,620)$ The crystal structures of A20 OTU with and without the active site cysteine (C103) converted to the sulfenic acid have been disclosed. Based on the crystal structure of the sulfenic acid, it was concluded that the ability of the active site to accommodate and stabilize the sulfenic acid protects against hyper-oxidation that would likely render oxidation irreversible.(621) This protection is hypothesized to arise because the hydroxyl group within the sulfenic acid can hydrogen bond effectively with the loop on which the oxidized active nucleophile, $\mathrm{C} 103$, resides. Interestingly, available crystal structures(621) indicate that similar stabilization is possible for the sulfenic acid in other OTU DUBs and evidence provided indicates that many OTU DUBs are sensitive to physiological $(1 \mu \mathrm{M}) \mathrm{H}_{2} \mathrm{O}_{2}$ on relatively short (15 min) time scales. Thus, redox sensing appears to be a conserved trait in this enzyme class. Furthermore, in the case of USP1, a DUB involved in DNA damage response/regulation, reversible inhibition of protease activity was observed upon $\mathrm{H}_{2} \mathrm{O}_{2}$ treatment. Similar inhibition of USP7 has also been observed.(622) Indeed, elevation in polyubiquitination species upon redox stress in yeast was ascribed to inhibition of a specific DUB, Ubp2 (a USP family member).(623)

Recently it has been shown that sulfenic acids in active sites can behave as active-site nucleophiles.(624,625) Sulfenic acids have also been implicated in several catalytic cycles including methionine sulfoxide reductase.(626) Thus, the possibility that the sulfenic acid is involved in catalysis should not be dismissed out of hand.

4.1.2 Formation of stable sulfenamide and sulfenylamide-Sulfenamides and sulfenylamides are relatively new players in protein regulation. It has been known for at least 40 years that sulfenic acids within proteins can be trapped by exogenous amine nucleophiles. Oxidized GAPDH was intercepted by benzylamine to form what was proposed to be a sulfenamide, although precise identification was not possible at that time. Subsequent studies showed that treatment of the model peptide PFVCG with $\mathrm{HOCl}$ formed intermolecular sulfenamide crosslinks.(627) Sulfonamides of GSH have also been reported. $(627,628)$

In later work, intramolecular sulfenylamides were reported to form in response to oxidative stress.(629) In most models, such sulfenylamides protect against sulfur hyperoxidation. In the case of PTP-1B, oxidation of the active-site cysteine is configured to promote formation of a sulfenylamide (Figure 12A). This unusual bond formation elicits a change in structure that renders the active-site cysteine resistant to further oxidation. However, upon addition of reducing agents, the oxidized active site cysteine is reactivated, a process that may also be assisted by the unusual conformation caused by the sulfenylamide.(630) Convincing mechanistic evidence for this pathway in cells has been provided, linking PTP activity in B lymphocytes to ROS in cell through the use of (among other experiments) ROS scavengers. (631)

The related protein, PTP- $\sigma$, is also regulated through formation of a sulfenylamide. Similar modes of protection against hyperoxidation have been observed in Bacillus subtilis OhrR and PTPa. Based on the above examples and unifying structural features between them, structural requirements for this specific bond formation have now been proposed.(632,633) A lysine-cysteine sulfenamide has also been reported in the crystal structure of sublactam 
bound thiol- $\beta$-lactamase.(634) It has also recently been proposed that a sulfenamide linkage is formed between a sulfur and an adjacent lysine in NemR.(635) This mechanism is believed to be how this transcription factor senses ${ }^{-} \mathrm{OCl}$.

An interesting comparative study between mouse and human methionine sulfoxide reductase A (Msr-A) was recently published.(636) The human version of Msr-A is much more resistant to hyperoxidation by micromolar peroxide than the mouse analog, although the proteins share almost $88 \%$ amino acid sequence identity. The authors trace this difference to a single residue located 5 amino acids from the C-terminus, M229 (a valine in the human enzyme). Mutation of mouse M229 to valine, or truncation of the last 5 residues of the Cterminus of the mouse enzyme (containing Met229) renders the mouse enzyme resistant to oxidation with no significant change in specific activity. The difference in oxidative stabilities is proposed to occur because M229 prevents formation of a sulfenylamide by altering the conformation of the backbone $\mathrm{N}-\mathrm{H}$ required to attack to sulfoxide.(637) This result indicates that redox regulation may vary between similar organisms, and further strengthens the notion that redox regulation is context dependent.

4.1.2.1 Sulfenamide formation in drug mechanisms: Sulfenamide formation is a critical step in the mechanism of the drug omeprazole (Figure 12B). This clinically approved drug is an inhibitor of the $\mathrm{H}^{+} / \mathrm{K}^{+}$-ATPase that suppresses stomach acid secretion. Omeprazole is a sulfoxide prodrug that rearranges in acid first to a sulfenic acid and then to a sulfenamide. The sulfenamide can bind to an ATPase and forms a disulfide cross-link to a specific sulfur, inhibiting the protein.(638)

4.1.3 Disulfide formation-The majority of disulfides in cells are formed in the ER. The ER is optimized for formation and exchange of disulfide bonds.(639) From a study of 4000 proteins, half of disulfide bonds were found in membrane proteins, and most others had a secretory sequence.(640) However, 5\% of disulfides were localized in the nucleus or cytoplasm.(641) Disulfide bond formation in the cytosol is probably elevated upon oxidative stress.(642) These disulfides are formed reversibly, distinguishing them from "structural" or inert disulfides.(643) This distinction has been addressed computationally. $(643,644)$

We have discussed PTP-1B sulfenylamide formation above. Some analogs of PTP-1B, such as PTEN,(645,646) SHP1/2(647) and Cdc25(648) do not form stable sulfenic acids upon oxidation, but rather have evolved a vicinal cysteine that forms a disulfide with the oxidized active-site cysteine. This bond formation also prevents further oxidation and facilitates reactivation.(649) Although this is an ideal system to evaluate "why" sulfenylamide vs. disulfide formation occurs, it seems there has been little progress.

One possible explanation for the different hyperoxidation mechanisms is control of kinetics of re-reduction. Interestingly, thioredoxin-1 (Trx1) can reduce both oxidized PTP-1B and PTEN at similar rates $\left[14 \mathrm{M}^{-1} \mathrm{~s}^{-1}(650)\right]$. Such a low second-order rate is worrisome as it seems not physiologically useful and is also close to the rate at which PTP-1B is oxidized $\left(20 \mathrm{M}^{-1} \mathrm{~S}^{-1}\right)$ and over an order of magnitude slower than the rate of oxidation of TrxR1 (600 $\mathrm{M}^{-1} \mathrm{~s}^{-1}$ ).(651) However, it cannot be ruled out that slow, controlled reduction is one possible reason for sulfenylamide formation in the first place. As mentioned in section 3, the 
"average enzyme's" $k_{\mathrm{cat}} / K_{\mathrm{m}}$ is $10^{5} \mathrm{M}^{-1} \mathrm{~s}^{-1}$ (60\% of enzymes fall within an order of magnitude of this value), making PTP1-B and PTEN reduction by Trx 1 an unusually inefficient process, despite being putatively linked to a signaling (i.e., a dynamic response) pathway.(353) Nevertheless, the re-reduction rate is much faster than the corresponding rates of reduction of PTP-1B and PTEN by small molecule thiols $\left(0.036 \mathrm{M}^{-1} \mathrm{~s}^{-1}\right.$ for GSH), possibly hinting that re-reduction is overall slow for these proteins by design. Unfortunately, no clear in vivo evidence using endogenous levels of the protein players has been offered thus far for enzymatic re-reduction of PTP-1B and PTEN by Trx 1 under oxidative stress either. There is evidence that Trx 1 could be involved in basal recycling of oxidized PTP-1B, because in Trx 1 knockout MEFs, PTP-1B oxidation levels are increased.(651) One way to reconcile these data could be that TrxR1 or a TrxR1-regulated protein could rapidly intercept intermediate oxidation products (e.g. the sulfenic acid) under low stress conditions, but this mechanism may fail during oxidative stress, allowing/necessitating formation of the sulfenylamide. Recent work using affinity capture/digest MS has indicated a modest increase in binding of Trx 1 (a protein known to bind oxidized proteins with higher affinity than reduced congeners) and Prx1 upon oxidation of PTEN,(652) giving some extra credence to the idea that Trx1 or Prx1 may re-reduce oxidized PTEN in cells, although a clear thread of logic from kinetics to in vivo evidence has yet to be fully established.(653) One general conclusion from the study is that the interactomes of reduced and oxidized PTEN are different, possibly indicating that oxidized PTEN has specific downstream signaling roles. Thus, the different conformations and subsequent interactome changes, ushered by sulfenic acid and disulfide formation, may explain the differential redox regulation of PTEN and PTP-1B.

Recently, apoptosis-inducing factor (AIF), a protein that translocates from the mitochondria to the cytosol/nucleus upon apoptosis(654), was also shown to re-reduce PTEN (a peripheral membrane/cytosol-localized protein).(655) Although this study did not provide kinetic constants for the reduction, the authors used knockdown of AIF to probe in vivo relevance of this interaction. It would be very interesting to compare second-order rate constants for Trx 1 reduction of PTEN and PTP-1B with that of AIF1-catalyzed PTEN reduction to examine the relative impacts of these two pathways on PTEN reduction.

\subsection{Glutathionylation and other mixed disulfide modifications}

This modification is found in most kingdoms of life from bacteria (mainly Gram-negative species that contain GSH) to humans.(656) $S$-glutathionylation can occur either as a consequence of nucleophilic attack by GSH on an oxidized protein or by the reverse process. (657) This can be uncatalyzed or enzyme catalyzed.(658) This process is most commonly associated with reversible inactivation of enzymatic activity,(659) however other interesting effects have been reported.(659)

The yeast ER chaperone Kar2 (analog of human Bip) was recently shown to be affected by glutathionylation.(660) In the absence of glutathionylation, Kar2 functions as an ATPdependent chaperone. Upon glutathionylation, ATP activity is inhibited, converting the enzyme to a holdase (an enzyme that binds unfolded proteins, maintaining solubility, but not actively refolding them). It was speculated that glutathionylation allows Kar2 to maintain 
client protein solubility until stress has subsided and active refolding can restart.

Interestingly, a similar effect was found for sulfenylation of BiP, indicating that these modifications may have overlapping phenotypic effects.(661) Such degeneracy/convergence may be one way to ensure sufficient upregulation of the desired phenotype/activity occurs.

Glutathionylation is also implicated in recovery from injury. HIF-1a is a proangiogenic transcriptional factor that is stabilized upon glutathionylation at C520. Upon knockdown of the deglutathionylating enzyme, glutaredoxin (Glrx or Grx), GSH adducts to HIF-1a increased in ischemic muscles and promoted regain of blood flow. These data ultimately may indicate that Glrx inhibition may promote revascularization.(662)

Finally, an interesting redox relay ultimately producing glutathionylated sarco/endoplasmic reticulum calcium $\left(\mathrm{Ca}^{2+}\right)$ ATPase (SERCA) was reported by Adachi et al. The endpoint of this pathway is decrease in intracellular $\mathrm{Ca}^{2+}$, relaxing muscle cells.(663) In this model, glutathionylation of SERCA was mediated by $\mathrm{ONOO}^{-}$oxidation of a specific sensing residue, C674. Importantly, signaling occurred in the presence of low micromolar $\mathrm{ONOO}^{-}$, required GSH, was reversed by DTT, and did not occur in the C674S mutant in vivo. Furthermore, the SERCA inhibitor thapsigargin suppressed the effect of peroxynitrite on muscle relaxation, thus peroxynitrite likely (directly or indirectly) downregulates SERCA activity. Suppression of relaxation in atherosclerotic tissue was similar to that observed in thapsigargin-treated native tissue and thapsigargin had little effect on atherosclerotic tissue. These data indicate that atherosclerotic tissues (known to have high levels of oxidative stress) have lost the ability to regulate SERCA through peroxynitrite formation. The authors proposed that a hyperoxidized SERCA state was formed in these tissues that was unable to form a mixed disulfide. These data as a whole offer quite compelling evidence for a glutathionylated SERCA intermediate. Recently, numerous other types of small-molecule thiol adduct have been reported. These include protein CoA adducts.(664) Since CoA is localized preferentially in the mitochondria vs. the cytosol [ $2-5 \mathrm{mM}$ versus $0.02-0.14$ $\mathrm{mM}(665)]$, this could constitute an organelle-specific redox modification. Perhaps the simplest disulfide is persulfide. This modification, although formally a disulfide, retains nucleophilicity and acidity (both likely enhanced relative to cysteine).(666)

\subsection{Changing of hands of sulfenic acids}

Many of the above sulfur species (e.g., disulfide, sulfenic acid, sulfenamide, sulfenylamide) can functionally interconvert, either inter or intramolecularly. We have referred to this process as changing of hands of information. There are several reasons why changing of hands may be beneficial: (1) the rate of enzyme reactions can be many orders of magnitude faster than non-catalyzed rates; (2) the involvement of enzymes allows for greater specificity both in terms of target protein and specific disulfides exchanged; (3) there is also greater scope for contextual regulation; (4) the process can be of higher fidelity (i.e., less interception/signal leakage and collateral damage); (5) signals from numerous first responders can potentially converge at the same downstream point, leading to further amplification. Obviously, there is also a penalty in terms of loss of diffusability and reduction in immediacy of signal transfer. In some organelles and specific regions of the cytoplasm, crowding can facilitate direct transfer of substrates from one protein directly to 
the next protein in the pathway without the need for free diffusion.(667) Thus, diffusion could be removed from the equation, depending on the locale where information relay occurs. Disulfide exchange has been implicated in signaling pathways involving the yeast peroxide sensor Orp1 whose oxidation triggers a disulfide exchange with the transcription factor Yap1 (change of hands), thus activating Yap1. (668) Other examples include Pap1(669) and STAT3.(670) As we touched on above, some authors have also proposed that Keap1-the master redox sensor of the cell—does not sense oxidants fast enough to account for downstream signaling that occurs upon oxidative stress.(66) An intermediary has thus been postulated.(671)

\subsection{Oxidation of methionine}

There appears to be a rich functional biochemistry of methionine oxidation that is slowly being elucidated.(525) MICAL-mediated oxidation of F-actin occurs at two specific residues, M44 and M47. This overall process leads to disassembly of actin filaments.(672) Methionine sulfoxide reductase B1 can specifically reduce oxidized actin, restoring actin polymerization.(523) By far the most common method to study methionine oxidation involves direct oxidation of proteins, although mutation to glutamine, that is believed to mimic oxidized methionine, has also been used. There is some indication that methionine oxidation may be promoted by near-by phosphorylation sites.(673) This could indicate that phosphorylation and methionine oxidation crosstalk, although much more work needs to be done in this area. Methionine oxidation has been implicated in protection of sensitive residues from oxidation: in a-2-macroglobulin exposure to an oxidative environment leads to methionine oxidation, which does not change activity.(674) Upon over exposure to oxidants, tryptophan oxidation occurs leading to inactivation. A similar conclusion was reached for glutamine synthetase.(675) Consistent with methionine serving an antioxidant role, substituting norleucine (a methionine isostere) for methionine in E. coli led to hypersensitization to oxidative stress.(676) Similarly, oxidation of Calcium/calmodulin $\left(\mathrm{Ca}^{2+} / \mathrm{CaM}\right)$-dependent protein kinase II at methionine can lead to protein activation. $(677,678)$ Other proteins implicated in regulation through methionine oxidation include, apolipoprotein A-1(679) and calcineurin.(680)

\subsection{Oxidation of cysteines to $-\mathrm{SO}_{2} \mathrm{H}$}

Sulfinic acid is formally a reversible modification (see section 3.9.1), although reversal does not occur without catalysis and there are very few enzymes that catalyze this process.(681) Sulfinic acids have varied roles in cell signaling, although they are usually grouped together with sulfonic acids as differentiation between the two forms is not trivial.

We have already discussed the role of sulfinic acids in peroxiredoxin chemistry. Another interesting example is DJ-1, an atypical peroxiredoxin-like peroxidase.(682) DJ-1 is a protein strongly linked with neuroprotection. For instance, loss of function mutations to DJ-1 can cause early onset Parkinson's disease.(683) Sulfinic acid formation at C106 promotes mitochondrial translocation and suppresses neuron-protective effects of DJ-1.(684) The C106 sulfinic acid has been characterized structurally.(683) Sulfinic acid modification is also known to regulate nitrile hydratase (heme or cobalt containing enzymes that catalyze hydrolysis of nitriles), (685) and matrilysin (a matrix metalloprotease).(686) 


\subsection{Oxidation of cysteines to $-\mathrm{SO}_{3} \mathrm{H}$}

Consistent with a trend to a more oxidizing environment as organisms age (discussed in section 3) and the fact that sulfonic acid is an irreversible modification, it is likely that protein $\mathrm{SO}_{3} \mathrm{H}$ accrues with age.(687) Clearly, cysteine sulfonic acid formation at numerous active-site cysteines elicits loss of function, but, we focus on oxidation-induced gain of function in this section.

Since sulfonic acid formation is irreversible, perhaps unsurprisingly, one of the first examples of sulfonic acid signaling was as a gain of function co-degron in the $\mathrm{N}$-end rule. The $\mathrm{N}$-end rule is a fascinating example of protein regulation that describes how the half-life of a protein is determined by its $\mathrm{N}$-terminus.(688) Some residues are considered destabilizing, whereas others are stabilizing, although this is highly context dependent.(689) Because of the requirements of the Kozak sequence, proteins are translated with an Nterminal methionine.(690) However, this residue is often(691) [80\% of the time in yeast(692)] removed by methionine amino peptidase, exposing proteins with a diverse array of N-termini.(693) Arginine is the prototypical N-terminal degron; a subset of destabilizing residues (aspartate and glutamate) require conjugation to arginine prior to degradation. It is also known that NO-catalyzed oxidation of cysteine (a stabilizing amino acid) can form the sulfinic or sulfonic acid forms that appear to mimic aspartate or glutamate as they usher $\mathrm{N}$ terminal arginine conjugation, stimulating degradation through the $\mathrm{N}$-end rule.(694)

Other examples of cysteine sulfonic acid performing a signaling role include yeast peroxiredoxin, where sulfonic acid formation at the active-site cysteine leads to loss of peroxidase activity but stimulates chaperone activity 4-fold.(695) Some cysteine oxidation events are linked to disease etiologies. Sulfoxide formation on C111 of human SOD is linked to toxic gain of function through enhanced proclivity to form aggregates. $(696,697)$ C111- $\mathrm{SO}_{3} \mathrm{H}$ has been identified in Lewy-body-like structures in a mouse model of familial amyotrophic lateral sclerosis.

\subsection{Protein alkylation by monofunctional RES}

Monofunctional RES are alkylating agents bearing one electrophilic moiety, such as nitroolefins (Figure 13A). Nitroolefins affect activities of several proteins including xanthine reductase [nitro-oleic acid, non-competitive, $\mathrm{IC}_{50}=0.5 \mu \mathrm{M}(698)$ ] and GAPDH (nitrolinoleate, $\mathrm{IC}_{50}=10 \mu \mathrm{M}$ ).(504) As stated in section 3.9.3, these modifications are typically reversible. This reversal usually requires addition of excess reducing agent. 12$\mathrm{NO}_{2}$-AA - a compound with many ascribed health promoting roles(699) - inhibits protein disulfide isomerase (PDI) reversibly.(700) This effect was time dependent, consistent with (slow) covalent-bond formation. Interestingly, PDI has two activities, disulfide isomerism and chaperone activity, and they are both inhibited at a similar rate. Nitroolefins are also crucial signaling molecules in insects.(701)

8-nitro cyclic (c)GMP, a product of RNS reaction with cGMP, can modify cGMP-dependent protein kinase PKG, causing irreversible enzyme activation.(702) This report is similar to earlier reports saying that 8- $\mathrm{NO}_{2}$-cGMP can alkylate and stimulate Keap1.(289,290) In the case of PKG, alkylation appeared to occur at two cysteine residues within the high-affinity 
cGMP binding domain. These experiments were carried out in lysates using 1-200 $\mu \mathrm{M}$ of 8$\mathrm{NO}_{2}$-cGMP.(702) Cellular concentration of 8- $\mathrm{NO}_{2}$-cGMP can range within 0.01-10 $\mu \mathrm{M}(703)$ and the authors were able to show weak labeling of the protein under these conditions that was not prevented by DTT.

1,2-quinones label protein thiols with a rate constant of around $10^{3} \mathrm{M}^{-1} \mathrm{~s}^{-1}$. The second order rate constant for 1,2-benzoquinone adduction to protein (BSA) is on the order of $10^{4}$ $\mathrm{M}^{-1} \mathrm{~s}^{-1}$ and is believed to proceed through thiol adduction.(699) The reaction with amine functions is $10^{5}$-fold slower.(699) One region where 1,2-quinone may be in abundance is the brain. There is in fact a correlation between protein quinone adduction and dopamine (Figure S3) induced cytotoxicity (dopamine oxidation can produce $o$-quinones).(704) Several proteins involved in energy production/distribution were identified as quinone reactive in aged rat brain. Several of these targets (lactate and malate dehydrogenase) were inhibited by quinone modification.(705) In a separate study, SOD2 was also labeled and inhibited by quinones.(706)

Finally, dietary ITCs affect a host of enzymes. Although many different activities have been ascribed to these important reactive units, they inhibit deubiquitinating enzymes likely through direct interaction with the catalytic thiol to form an acyl-enzyme intermediate.(333)

4.7.1 Toxic activity of RES induced by xenobiotic metabolism-The action of CYP450s on aromatic species can create several toxic metabolites. This activity can be the principal cause of cytotoxicity of some drugs/xenobiotics. For instance, paracetamol's toxicity has been traced to formation of a reactive quinone imine through metabolic oxidation. $(707,708)$ Benzene toxicity is also likely the result of metabolic processes forming benzene oxide and also quinones.(709) Tobacco smoke contains many polyaromatic hydrocarbons that can upregulate CYP450 (including CYP1A1 and CYP1A2 isoforms)(710) and promote their toxicity (this is principally catalyzed by CYP2A6 and -13$).(709,711)$ It is possible that this metabolic toxicity is aggravated in HIV patients who are smokers.(712) Indeed, CYP450 inhibitors reduce toxic/carcinogenic compounds formed from tobacco smoke. For instance, it has been proposed that PEITC, a time-dependent inhibitor of several CYP450 isoforms, including CYP1A2 $\left(K_{i}=10 \mu \mathrm{M}\right)$ and CYP2A6 $\left(K_{i}=19 \mu \mathrm{M}\right)$, may elicit some of its anticancer properties by targeting this enzyme and reducing toxic by-products formed, especially in smokers.(713)

\subsection{Protein alkylation by multifunctional RES}

Multifunctional RES have wider scope to affect protein activity/stability. This is because they can bind to two separate sites (cross-linking) and potentially induce unexpected protein conformations, for example. (Figure 13B). Unsurprisingly, early work linked protein HNEylation to decreased protein stability. This was attributed to 20S proteasome activity, independent of the 19S chaperone, at low HNE concentrations.(714) At higher concentrations, HNE can inhibit the proteasome, possibly through direct interaction(715) and/or because some HNEylated proteins may inhibit the proteasome directly.(716) Several studies have highlighted the link between bifunctional RES and several diseases.(271) 
Consistent with its ability to engender severe negative effects on protein structure/behavior, HNE is associated with aggregation of numerous proteins in vitro and in cells. $(717,718)$ However, other work shows that HNE can form defined adducts with specific proteins; such adducts may be reversible/reactivatable. $\mathrm{Na}^{+}-\mathrm{K}^{+}$ATPase was inhibited by HNE with an $\mathrm{IC}_{50}=120 \mu \mathrm{M}$ irreversibly under the assay conditions. Importantly, activity was restored upon incubation with both hydroxylamine and a reducing agent; reducing agent alone had no effect.(502) Glutathione peroxidase is inhibited by HNE with an $\mathrm{IC}_{50}=0.12 \mathrm{mM}$. Inhibition can be prevented, or reversed by addition of glutathione, but not DTT or $\beta$-mercaptoethanol. (719) Interestingly, HNE adduction can also occur on cysteines that are apparently buried in the static crystal structure.(720) Adipocyte FA binding protein, a protein that regulates adipocyte FA uptake, is a specific target of HNE. Binding of either enantiomer of HNE to a specific cysteine (C117) inhibited FA binding to this protein by 10-fold.(721) This HNEprotein complex was later analyzed by x-ray crystallography, showing that HNE bound in the oleic acid binding pocket.(722) Interestingly, the overall fold and structure of the protein is remarkably similar to that of the apoprotein, proving that HNE can bind proteins without eliciting severe perturbations in structure.

Bifunctional RES can also perform signaling roles.(723) Indeed, HNE signaling has now been linked to a host of signaling responses (often via modification of specific proteins) including stress signaling,(724) apoptosis,(725) and regulation of mitochondrial uncoupling. (726) HNE is also linked to numerous diseases(255) and also numerous eustress pathways. $(727,728)$ For instance, preconditioning with low $(15 \mu \mathrm{M}) \mathrm{HNE}$ prior to treatment with lethal doses of different cytotoxic compounds (e.g., $\mathrm{H}_{2} \mathrm{O}_{2}$ ) is cytoprotective to PC12 cells. (727) Thioredoxin reductase 1 (TrxR1) was implicated in this pathway.

\subsection{RES-containing drugs and compounds in clinical trials}

There is a rich and diverse array of drugs in clinical trials and clinical use that take advantage of reactive carbonyl functions, such as acrylamide. We will define such drugs as RES-containing drugs ("RES drugs"). Although this is not the forum for an extensive thesis, we point out a few aspects of note. RES drugs fall into three main classes: (I) active-site targeting RES drugs; (II) non-active-site targeting RES; (III) RES formed upon mechanismbased inactivation.(390) Clinical examples of each class are: carfilzomib, the epoxide-based proteasome inhibitor [ $\left.k_{\text {inact }} / K_{\mathrm{i}} 34,000 \mathrm{M}^{-1} \mathrm{~s}^{-1}(729)\right]$; Dacomitinib, an epidermal growth factor receptor (EGFR) kinase inhibitor bearing an acrylamide function $\left[k_{\text {inact }} / K_{\mathrm{i}} 10^{6} \mathrm{M}^{-1} \mathrm{~S}\right.$ $\left.{ }^{-1}(730)\right]$; and Gemzar, a suicide inactivator of ribonucleotide reductase, that forms a cross link to the enzyme. $(731,732)$ Critically, for inherently-reactive irreversible binders that are not generated in situ, i.e., Class I and II, these must (ideally) react slowly with background thiols but fast with the intended substrates, to enable pharmacologically-relevant occupancy of the target prior to degradation/excretion. Rapid labeling kinetics is engendered through binding to a specific pocket on the target enzyme, facilitating an otherwise slow bondforming step [second-order rate of thiol addition to epoxide is $0.001 \mathrm{M}^{-1} \mathrm{~s}^{-1}$ and the same rate is $0.021 \mathrm{M}^{-1} \mathrm{~s}^{-1}$ to acrylamide, for instance $\left.(733,734)\right]$. Thus, very high rate accelerations are required to engender the second-order rate constants of inhibition for carfilzomib and Dacomitinib mentioned above. Moreover, these drugs show rate enhancements on the order of $10^{7}$ for reaction with their target relative to the reaction with 
GSH, indicating that in spite of the high GSH content in cells, the reaction with the enzyme may be many orders of magnitude faster.

Histidine is also a possible target of drugs. Fumagillin (735) and its analog Beloranib [a compound withdrawn from clinical trials (736)] alkylate methionine aminopeptidease at an essential histidine. Interestingly, numerous chloroketones alkylate serine proteases at histidine, despite the active site residue being serine.(737) This regioselectivity can be explained because the catalytic serine rapidly attacks the carbonyl group within the inhibitor, leaving only the histidine (part of the catalytic triad) to react with the chloride.(738)

Some ligands target reactive nucleophiles on threonine/serine. In this instance, a clever way to side-step competing cellular thiols and provide selectivity is to use ligands that bind reversibly to thiols but will form more stable bonds to target oxygen nucleophiles. Boronates (structural analogs of carboxylic acid/amides) fit this bill perfectly, and indeed bortezomib, a boronate-based peptide drug, has emerged to be a highly selective proteasome inhibitor used clinically.(739)

Ironically, one of the limiting factors of bortezomib is its low volume of distribution due to rapid adsorption by blood proteasomes. This limits bortezomib's tissue penetration.

Millennium designed a second-generation boronate proteasome inhibitor drug that has a faster off-rate, allowing dissociation from blood proteasomes, and affording much more tissue penetration.(740) Clearly, these examples illustrate the importance of pharmacokinetics in drug efficacy and design. Indeed, irreversible binding is one way to sustain drug action long after clearance, a trait that to some extent can uncouple pharmacokinetics from pharmacodynamics. However, there is a dark side of covalent drug design: off-target labeling accrues with time, in a similar way to HNE reacting with first responders vs. non-activated proteins. The Cravatt laboratory has disclosed a method to minimize off-target effects by designing an electrophilic appendage that is deactivated faster than off-target binding events can occur.(741) Finally, mechanism-based inhibitors do not really conform to these rules because a very reactive electrophile is generated only within the active site, meaning trapping is quite efficient. Given that there are no HT screens for mechanism-based inhibition, most screening has focused on ligandable cysteines (Classes I and II).

Redox biology and covalent drug discovery have a lot to learn from one another. In effect, redox signaling at privileged sensors is not so distinct from the way a Class I or II covalent drug functions, especially with the growing use of encapsulation using polymer carriers, (742) dendrimers and fullerenes(743) as well as antibody conjugation.(744) Clearly, the discussion of covalent drug mechanisms does show that binding can lead to elevated secondorder rates, and that even seemingly reactive warheads can give sufficient efficacy for pharmaceutical benefit (see Section 6). Thus, given sufficient evolutionary pressure, it is certainly possible that enzyme-like redox sensors have arisen. Of course, pharmacologic efficacy does not necessarily mandate high selectivity, just that there be a beneficial output. Thus, a drug's efficacy is an amalgam of effects due to all beneficial and detrimental binding events.(745) 
However, the average number of known (and hence possibly an underestimate of total binders) targets per approved drug on average is relatively low $(<5)$, arguing that too promiscuous interactors may be deleterious. In fact, comparative analysis of approved and withdrawn drugs indicates that it is number of off-target proteins that are essential genes that may cut the line between success and failure of a drug.(746) Such an analysis has not been applied to redox signaling, but one could postulate that a similar trend may be observed (i.e. that endogenous electrophiles would have a low number of essential gene hits under "signaling conditions").

\section{METHODS TO INTERROGATE RES/ROS SIGNALING}

\subsection{Detecting RES/ROS generation in cells and organisms}

The generation of RES/ROS signals is tightly controlled both spatially and temporally. One of the key challenges in deconstructing RES/ROS signaling has been in defining where and when these signals are generated.(747) For this reason, many techniques we will discuss rely on whole cell or organism imaging. Regardless of the readout, an ideal probe to detect each signal chemotype must be able to measure the dynamics of signal generation in real time and must meet at minimum the following criteria:

1. Chemoselectivity: the probe should react only with the specific RES/ROS signal. Obviously, less specific probes can provide a "general" idea of "redox changes" in a cell and have their uses, but ultimately low chemoselectivity is less useful for mechanistic studies. Importantly, chemically-similar molecules can be present in cells at widely-different concentrations and the selectivities must reflect not only intrinsic rate differences, but also concentration bias: it is useless to have a sensor with 10-fold selectively for metabolite A over B if concentration of B is 1000fold in excess of A.

2. Sensitivity: some reactive species, such as peroxynitrite, are present in cells in low (nanomolar/picomolar) quantities (Table 1),(70,73) so sensing power must be significant. In addition, a broad linear range, capable of detecting a wide range of RES/ROS concentrations encountered in both cells and organisms is also desirable.

3. Signal-to-noise-ratio: native/unreacted probe must have low background signal.

4. Membrane permeability: should be permeable to cellular membrane(s) if measuring intracellular or in vivo generated signals

5. Biorthogonality: the probe should be (as) non-intrusive (as possible).

6. Reversibility: a reversible probe will allow real-time monitoring of RES/ROS flux, rendering it ideal for ultimately relating these measurements to specific signaling events. Conversely, an irreversible probe may be able to cumulatively detect signals, allowing a weaker signal to be detected, but this comes at the expense of losing dynamic information. 
7. Ratiometric: a ratiometric probe enables measurement of small changes in signal concentrations and eliminates artifacts due to variations in loading/retention of the probe.

8. Probe must not be affected by the unique cellular environments, i.e., stable/ unaffected across the desired $\mathrm{pH}$ range(s), ionic strength(s), hydrophobicity(ies).

Many of the modern methods to monitor the generation of ROS address most of the points above. Although most methods still have caveats, the techniques themselves are powerful when used/controlled appropriately. However, equivalent methods to detect RES are underdeveloped. This is partly because LDEs classes can contain many different chemical structures but have very similar reactive chemical groups, rendering chemo selectivity a huge issue. These traits are in stark contrast with oxidative ROS-based signals that differ in terms of charge, and type of chemistry (e.g., single vs two electron; nucleophilic addition vs [4+2] cycloaddition/ene) they can undertake. LDEs also react with multiple different amino acids including cysteine, histidine, and lysine generating an increasing diversity of protein adducts, most of which probably retain RES-like properties. Thus, it is challenging even to design a probe for a specific RES that will not also intercept protein RES adducts. As we will see, ROS sensors have been developed that take advantage of the many enzymes that interact with biological oxidants (semi)-selectively. Many of these ROS-sensitive proteins have been harnessed in sensor development. However, relatively few proteins are known to react with RES selectively, and hence enzymatic RES sensing is very poorly developed, although we will discuss an example in subsequent sections. In the following subsections, we will discuss and compare various methods that allow measurement of RES and ROS generation. We will evaluate each probe using the eight-point criteria above.

\subsubsection{Small-molecule fluorescent probes to detect ROS-Small molecule} fluorescent probes are commonly deployed for redox signal detection.(748-750) A list of commonly used probes to detect endogenously-generated ROS is provided in Table 3 . Fluorescent probes utilize the redox reaction between ROS and a dye to monitor changes in ROS (or several similar ROS). Such readouts are possible because oxidation of the dye increases fluorescence. Because the probe is irreversibly oxidized, fluorescent probes do not typically permit measuring dynamic changes of redox signals in cells. Additionally, this feature of fluorescent-based probes also implies that these sensors alter the redox state/ equilibria of the cell by acting as ROS scavengers.

One of the earliest and most commonly used probes in detection of oxidants is dichlorodihydrofluorescein $\left(\mathrm{DCFH}_{2}\right)$ and its ester, dichlorodihydrofluorescein diester $\left(\mathrm{DCFH}_{2}\right.$-DA) (Figure 14A).(751) The diester is more readily cell-permeable and gets cleaved by intracellular esterases to generate $\mathrm{DCFH}_{2}$. $\mathrm{DCFH}_{2}$ reacts with intracellular oxidants to generate the fluorescent molecule DCF. Both 2 electron and stepwise single electron oxidation have been proposed for $\mathrm{DCFH}_{2}$ activation and it is likely that both singleelectron acceptors (such as radicals) and two-electron acceptors can oxidize this probe. $(751,752)$ Thus, $\mathrm{DCFH}_{2}$ is best used to quantify general oxidative stress, and it does not report on changes to a specific oxidant. $\mathrm{DCFH}_{2}$ has been widely used in cell-based experiments to measure what was typically assumed to be " $\mathrm{H}_{2} \mathrm{O}_{2}$ " generation. $\mathrm{DCFH}_{2}$ has 
been used to measure the respiratory burst of oxidants, referred to as "peroxide", in neutrophils, $(753,754)$ and macrophages.(755) In fact, it is now established that the probe is not particularly reactive with $\mathrm{H}_{2} \mathrm{O}_{2}$. $(751,752)$ Rather, the reaction of $\mathrm{H}_{2} \mathrm{O}_{2}$ with $\mathrm{DCFH}_{2}$ is enabled by the presence of trace metals and enzyme catalysts, including peroxidases,(756) that generates other ROS such as ${ }^{\circ} \mathrm{OH}$. ${ }^{\circ} \mathrm{OH}$ reacts with $\mathrm{DCFH}_{2}$ with a rate constant of $1.3 \times 10^{10} \mathrm{M}^{-1} \mathrm{~s}^{-1}$.(757) ${ }^{\circ} \mathrm{OH}$ scavengers such as DMSO and mannitol partially inhibit oxidation of the probe, further validating the role of ${ }^{\circ} \mathrm{OH}$ as the oxidant responsible for $\mathrm{DCFH}_{2}$ oxidation.(758) In a comparative study, millimolar peroxide was around 100 times less potent at stimulating $\mathrm{DCFH}_{2}$ fluorescence than alkyl peroxides, even though the alkyl peroxides were present in the micromolar range.(759) $\mathrm{DCFH}_{2}$ is also oxidized by ${ }^{1} \mathrm{O}_{2}(760)$ and $\mathrm{ONOO}^{-}(761)$ although the mechanism of oxidation by the latter is not well understood. The lack of specificity makes $\mathrm{DCFH}_{2}$ a useful tool for measuring broad increases in intracellular ROS such as those under oxidative stress conditions. However, its use in detecting specific ROS chemotypes under redox signaling conditions is limited and therefore caution must be exercised when interpreting data obtained using $\mathrm{DCFH}_{2}$.

New derivatives of $\mathrm{DCFH}_{2}$ such as $\mathrm{HK}-$ Green possess enhanced selectivity towards $\mathrm{ONOO}^{-}$ over ${ }^{\circ} \mathrm{NO},{ }^{1} \mathrm{O}_{2}, \mathrm{O}_{2}{ }^{\bullet-}$, and ${ }^{\bullet} \mathrm{OH}$ and ${ }^{\circ} \mathrm{OR}$ in cell-free systems (Figure 14A).(762) Firstgeneration HK-Green1 showed a marginal(763) (and likely not biologically significant) 2-3 fold selectivity towards $\mathrm{ONOO}^{-}$over its secondary metabolites nitrogen dioxide radical $\left({ }^{\circ} \mathrm{NO}_{2}\right)$, carbonate radical $\left(\mathrm{CO}_{3}{ }^{-}-8\right)$, and ${ }^{\circ} \mathrm{OH}$. HK-Green1 was used for the detection of $\mathrm{ONOO}^{-}$in neuronal cells. Fluorescence signal intensity was only observed in neuronal cells treated with the $\mathrm{ONOO}^{-}$donor, 5-amino-3-(4-morpholinyl)-1,2,3-oxadiazolium chloride (also called SIN-1), but not with the NO donor, S-Nitroso-N-Acetyl-D, L-Penicillamine (also called SNAP) or the $\mathrm{O}_{2}{ }^{-}$-inducer xanthine/xanthine oxidase.(763) Next generation HK-Green probes (HK-Green4) have significantly enhanced the selectivity of the probe ( $\sim 300$ fold) towards $\mathrm{ONOO}^{-}$over its secondary metabolites. The limit of detection for HKGreen4 is estimated to be $\sim 10 \mathrm{nM}$.(762) However, this limit may actually be an order of magnitude too high to measure endogenous peroxynitrite in some cells. $(70,73,764)$ Nevertheless, HK-Green 4 was recently used to measure $\mathrm{ONOO}^{-}$generation upon stimulation of macrophages RAW264.7 cells and primary mouse macrophages demonstrating its use in measuring physiologically accessible concentrations.(762) Additionally, HK-Green 4 could be used for two-photon imaging of $\mathrm{ONOO}^{-}$in macrophages $\left(\lambda_{\mathrm{ex}}=730 \mathrm{~nm}, \lambda_{\mathrm{em}}=500-550 \mathrm{~nm}\right)$ making it a potentially useful tool for in vivo experimentation. Another useful probe for the detection of highly reactive ROS was developed by the Nagano lab.(765,766) 2-[6-(4'-amino)phenoxy-3H-xanthen-3-on-9yl]benzoic acid (APF) and 2-[6-(4'-hydroxy)phenoxy-3H-xanthen-3-on-9-yl]benzoic acid (HPF) selectively detect highly reactive oxygen species such as ${ }^{\circ} \mathrm{OH}, \mathrm{ONOO}^{-}$, and $\mathrm{HOCl}$ (>50-fold selectivity) compared to ${ }^{1} \mathrm{O}_{2}, \mathrm{O}_{2}{ }^{--}$and $\mathrm{H}_{2} \mathrm{O}_{2} \cdot(766)$

Dihydrorhodamine (DHR) and Hydroethidine (HE) possess similar properties to that of $\mathrm{DCFH}_{2}$ and have also been used to measure ROS fluxes in cells (Figure 14A). Like $\mathrm{DCFH}_{2}$, DHR does not react directly with $\mathrm{O}_{2}{ }^{--}$and $\mathrm{H}_{2} \mathrm{O}_{2}$. (767) However, it readily reacts with ${ }^{\circ} \mathrm{OH}$ with rate constants close to the diffusion-controlled limit.(757) DHR is also oxidized by $\mathrm{HOCl}(768)$ and by $\mathrm{ONOO}^{-}$indirectly through the radical decomposition products, ${ }^{\circ} \mathrm{NO}_{2}$, and $\mathrm{CO}_{3}{ }^{-}$7.(757) The rate constants for the reaction of $\mathrm{CO}_{3}{ }^{--}$and ${ }^{\circ} \mathrm{NO}_{2}$ with DHR are both 
on the order of $6 \times 10^{8} \mathrm{M}^{-1} \mathrm{~s}^{-1}$.(757) $\mathrm{HE}$, on the other hand, is more selective towards $\mathrm{O}_{2}{ }^{--}$ (over $\mathrm{CO}_{3}{ }^{\circ-}$ and ${ }^{\circ} \mathrm{NO}_{2}$ ) and reacts with a rate constant of $\sim 10^{6} \mathrm{M}^{-1} \mathrm{~s}^{-1}$. (769) HE generates 2OH-E $\mathrm{E}^{+}$upon oxidation with $\mathrm{O}_{2}^{--}$(Figure 14B). Recent reports, however, have cautioned regarding the absolute selectivity of $\mathrm{HE}$ towards $\mathrm{O}_{2}{ }^{--}$, demonstrating that $\mathrm{HE}$ can react with other oxidants such as ${ }^{\circ} \mathrm{OH}$ and $\mathrm{HOCl}$.(770) Nonetheless, the rates of oxidation with these oxidants in comparison to $\mathrm{O}_{2}{ }^{-}$have not been well characterized. Interestingly, none of the other biologically-relevant oxidants form the fluorescent $2-\mathrm{OH}-\mathrm{E}^{+}$upon reaction with HE. Instead, these non-specific oxidations generate ethidium $\left(\mathrm{E}^{+}\right)$. The fluorescence spectra of $\mathrm{E}$ + and 2-OH-E ${ }^{+}$overlap which may confound data interpretation(770) by confocal microscopy or flow cytometry methods. $(769,771)$ Careful use of selective excitation wavelengths to distinguish the $\mathrm{O}_{2}{ }^{-}-$-dependent $2-\mathrm{OH}-\mathrm{E}^{+}$generation $(385-405 \mathrm{~nm})$ from E $+(480-520 \mathrm{~nm})$ mitigates this issue.(772) Alternatively, high-pressure liquid chromatography (HPLC) can be used to separate and quantify $2-\mathrm{OH}-\mathrm{E}^{+}$as a means to faithfully report on the generation of $\mathrm{O}_{2}{ }^{-}$in biological systems.(771) $\mathrm{HE}$ and its mitochondria-targeted analog (Mito $\mathrm{HE}$ or Mito-SOX) have been widely used for $\mathrm{O}_{2}{ }^{--}$ detection in cells.(773-775) One point of concern underlying the use of HE (or any probe for $\mathrm{O}_{2}{ }^{-}$- detection) is that the probe must compete with SODs that are present at micromolar concentrations in a cell.(19) The second-order rate constant for reaction of $\mathrm{O}_{2}{ }^{--}$with SODs is $\sim 10^{9} \mathrm{M}^{-1} \mathrm{~s}^{-1}$,(103) suggesting that around $1 \mathrm{mM}$ intracellular concentration of the probe will be required for efficient detection of $\mathrm{O}_{2}{ }^{--}$generation in cells.(771)

Amplex Red is yet another small-molecule-based reporter widely used for $\mathrm{H}_{2} \mathrm{O}_{2}$ detection (Figure 14C). Amplex Red is colorless, but upon oxidization generates brightly fluorescent Resorufin. This oxidation of Amplex Red by $\mathrm{H}_{2} \mathrm{O}_{2}$ requires the activity of a peroxidase enzyme to form a dye-based radical thus limiting its use in detection of intracellular $\mathrm{H}_{2} \mathrm{O}_{2}$ generation, although radical generation occurs at a rate greater than $1.9 \times 10^{6} \mathrm{M}^{-1} \mathrm{~s}^{-1}$ (with HRP as the catalyst).(776) Amplex Red has been used to detect generation of extracellular $\mathrm{H}_{2} \mathrm{O}_{2}$. The $\mathrm{H}_{2} \mathrm{O}_{2}$ detected is formed by spontaneous dismutation of $\mathrm{O}_{2}{ }^{--}$, generated by NOX and DUOX. The sensitivity threshold for Amplex Red in $\mathrm{H}_{2} \mathrm{O}_{2}$ detection is reportedly 50 nM.(777) However, at high concentrations of $\mathrm{H}_{2} \mathrm{O}_{2}$ and when the concentration of Amplex Red is limiting, the product, Resorufin, becomes further oxidized by peroxidase to generate non-fluorescent products.(778) Care must also be taken when deconvoluting data from Amplex Red since various endogenous metabolites such as GSH and NADH interfere with the signal generated from Amplex Red.(779) Additionally, $\mathrm{O}_{2}{ }^{--}$directly oxidizes Amplex Red, thereby further complicating the readout. Addition of SODs has been suggested as a strategy to circumvent these issues, although this will perturb normal cellular redox regulation and may cause additional artifacts. $(779,780)$ Interestingly, reduction of resazurin [alamarBlue (781)] to resorufin (the same fluorescent product of Amplex Red oxidation; Figure 14C) has been a popular cell viability assay for many years, although the mechanism of intracellular resazurin reduction to resorufin remains poorly understood.

Small molecule $\mathrm{HOCl}$ sensors have been known for some years. These are all turn-on fluorescence probes. Early examples of nanoparticle-based sensors showed utility in mouse splenocytes and tissues.(782) However, these probes were unable to distinguish $\mathrm{HOCl}$ from $\mathrm{ONOO}^{-}$. Another small-molecule probe (MMSiR) was more selective, showing many fold selectivity for $\mathrm{HOCl}$ over ${ }^{\circ} \mathrm{OH}, \mathrm{ONOO}^{-}$, and peroxide amongst others.(783) This probe was 
able to show elevation of $\mathrm{HOCl}$ in neutrophils upon phagocytosis. A subsequent BODIPYbased sensor, HCSe, was developed.(784) HCSe is not fluorescent in the reduced state due to photoinduced electron transfer (PET) from a pendant diphenyl selenium moiety to the BODIPY ring. Upon Se oxidation, PET is suppressed and a large increase in fluorescence is observed. Oxidation is highly selective for $\mathrm{HOCl}$ over numerous other biological oxidants. As selenium oxidation is reversible, $\mathrm{HCSe}$ was shown to be a reversible $\mathrm{HOCl}$ sensor in cells.(784) Recent years have seen two-photon probes developed with sensitivity thresholds as low as $20 \mathrm{nM} .(785)$

\subsubsection{Small-molecule boronate probes to detect $\mathrm{H}_{2} \mathrm{O}_{2}$-Aryl boronates take} advantage of chemistry known since the late 1950's(795): $\mathrm{H}_{2} \mathrm{O}_{2}$ can act as a two-electron donor to the boron within the aryl boronate probe while simultaneously behaving as an oxidant (electron sink) for (migration of) boron aryl or alkyl groups (Figure 15).

Nucleophilic attack of $\mathrm{H}_{2} \mathrm{O}_{2}$ to the electrophilic aryl boronate forms a transient tetrahedral boron "ate" complex, capable of undergoing a 1,2 shift of the C-B bond and forming a C-O bond, and re-establishing planarity of the boron atom. Hydrolysis of the boronate generates boric acid and liberates the fluorophore(796) (Figure 15B). This process converts the boronate functional group, which is not fluorescent, to the fluorescent phenol.

Aryl boronate probes are selective towards $\mathrm{H}_{2} \mathrm{O}_{2}$ over other endogenous ROS such as ${ }^{\circ} \mathrm{OH}$, ${ }^{\circ} \mathrm{NO}, \mathrm{O}_{2}{ }^{\circ}$ and ${ }^{\circ} \mathrm{OR}$ (797) as these reagents are 1 electron donors and cannot form the active boron "ate" species, nor do they have a good leaving group to promote translocation. However, recent studies show aryl boron probes can react with $\mathrm{ONOO}^{-}$and $\mathrm{HOCl}$.(789) Unsurprisingly, both these products are ideal for 1,2-migration chemistry and can engage productively to form boron "ate" complexes isolobal with peroxide boron "ate" complexes. By analogy, any similar (isolobal) chemical function, e.g. alkyl peroxides, will also be detected by the probe.(798) In fact, the second-order rate constant for the reaction of 4acetylphenylboronic acid with $\mathrm{ONOO}^{-}$is a million times $\left[k=1.6 \times 10^{6} \mathrm{M}^{-1} \mathrm{~s}^{-1}\right.$; typical concentration $1 \mathrm{nM}(71,73)]$ faster than that with $\mathrm{H}_{2} \mathrm{O}_{2}\left[k=2.2 \mathrm{M}^{-1} \mathrm{~s}^{-1} ; 1 \mathrm{nM}-700 \mathrm{nM}\right.$ in cells(24)] and almost 200 times faster with $\mathrm{HOCl}\left(k=6.2 \times 10^{3} \mathrm{M}^{-1} \mathrm{~s}^{-1}\right)$.(789) However, the fact that both $\mathrm{ONOO}^{-}$and $\mathrm{HOCl}$ have shorter intrinsic half-lives and are present in lower quantities in most cells than $\mathrm{H}_{2} \mathrm{O}_{2}$ (especially when ROS signaling occurs) may be sufficient to offset the kinetic differences for semi-compartmentalized processes. But as with any chemical probe, caution must be exercised to obtain the most meaningful results.

The first-generation probe Peroxyfluor-1 (PF-1) used two protecting aryl boronate groups (Figure 15A). The setup provides a high signal-to-noise ratio.(788) However, PF-1 suffers from slow turn-on kinetics since it requires two equivalents of $\mathrm{H}_{2} \mathrm{O}_{2}$ per molecule of PF-1 to elicit maximal response. Recently, a number of monoboronate-based probes including Peroxy Green-1 (PG1), PF-2, 3, and 6, Peroxy Yellow 1, and Peroxy Orange 1 (PO1) have been developed for application in intracellular detection of $\mathrm{H}_{2} \mathrm{O}_{2}$. (796) Although these probes are very useful, it should be stated that typically exogenous application of the oxidant or stimulation is required to detect the specific ROS. PG1 has been used to detect endogenous $\mathrm{H}_{2} \mathrm{O}_{2}$ generated upon EGF stimulation in A431 as well as neuronal cells.(790) The availability of these probes in a color palette also allows concurrent detection of $\mathrm{H}_{2} \mathrm{O}_{2}$ and other ROS using orthogonal fluorescent probes by dual color imaging. An example 
includes the use of PO1 $\left(\lambda_{\text {ex }} 507 \mathrm{~nm} ; \lambda_{\mathrm{em}} 574 \mathrm{~nm}\right)$ and $\operatorname{APF}\left(\lambda_{\mathrm{ex}} 490 \mathrm{~nm} ; \lambda_{\mathrm{em}} 515 \mathrm{~nm}\right)$ in RAW264.7 cells to simultaneously detect the generation of $\mathrm{H}_{2} \mathrm{O}_{2}$ and other ROS upon stimulation with phorbol myristate acetate (PMA), an inducer of phagocytosis-associated peroxide generation.(799) Further modification of boronate probes to engender enhanced retention in cells has enabled fluorescence intensity detection in HeLa cells upon stimulation with as low as $10 \mu \mathrm{M} \mathrm{H}_{2} \mathrm{O}_{2}$.(797) Nevertheless, exogenous stimulation is still required to detect robust signals. PF- 6 was also able to detect $\mathrm{H}_{2} \mathrm{O}_{2}$ generation by hippocampal stem/ progenitor cells upon FGF-2 stimulation.(797)

It must be stressed that although there are exceptions, a general limitation with chemical probes is that they are not sensitive enough to measure basal changes in peroxide, and are most commonly used to measure peroxide levels in cells treated with exogenous peroxide.

\subsubsection{Ratiometric small-molecule boronate probes to detect $\mathrm{H}_{2} \mathrm{O}_{2}$-Although} aryl boronate probes are semi-selective toward $\mathrm{H}_{2} \mathrm{O}_{2}$, these molecules have proven not well suited to provide a quantitative assessment of $\mathrm{H}_{2} \mathrm{O}_{2}$ in whole organisms. Variations in local probe concentration, probe diffusability, thickness of the sample, as well as changes in cellular microenvironment can significantly influence quantitation of signal generated by the probe. To obviate these issues, a number of studies have reported the development of ratiometric probes. Ratiometric sensors have the advantage of not being influenced by concentration of the sensor, meaning that small changes in redox environment can be measured accurately, with fewer numbers of cells. Ratio-Peroxyfluor 1 (RPF1) uses FRET between a coumarin donor and a boronate-caged fluorescein acceptor to quantitatively report on the generation of $\mathrm{H}_{2} \mathrm{O}_{2}$ in intact isolated mitochondria (Figure 15A).(792) Yet another ratiometric probe, Peroxy Lucifer 1 (PL1), uses a boronate-based switch to detect $\mathrm{H}_{2} \mathrm{O}_{2}$ by controlling internal charge transfer within 1,8-naphthalimide dye.(793) Upon reaction with $\mathrm{H}_{2} \mathrm{O}_{2}$, the emission maxima changes from blue to green. $\mathrm{PL} 1$ was able to detect $\mathrm{H}_{2} \mathrm{O}_{2}$ generation in phagocytic vesicles upon PMA treatment of RAW264.7 cells using two-photon illumination $(820 \mathrm{~nm})$. Recent advances have furthered the use of organelle-localized $\mathrm{H}_{2} \mathrm{O}_{2}$ sensors. A nuclear-localized aryl boronate probe, NucPE1, was used to show that overexpression of Sir-2.1 in C. elegans results in a concomitant decrease in $\mathrm{H}_{2} \mathrm{O}_{2}$ flux in the nucleus (Figure 15A).(794) Additionally, several mitochondria-targeted probes including Mitochondrial Peroxy Yellow 1 (MitoPY1),(800) SHP-Mito,(801) and MitoBoronic acid (MitoB)(802) have also been generated mainly by utilizing a triphenyl phosphonium moiety for mitochondrial targeting.(803) Recently, the use of SNAP labeling technology has enabled localization of boronate-based probes to the plasma membrane, mitochondria, nucleus, and the ER.(804)

Another problem with boronate-based probes (as with other fluorescent-based probes mentioned above) is that they undergo irreversible oxidation with $\mathrm{H}_{2} \mathrm{O}_{2}$. Some work has tried to mitigate this problem by developing reversible fluorescence-based redox sensors. Redox Fluor 1 (RF1) and its acetoxymethyl ester derivative RF1-AM, use a disulfide switch coupled to a fluorescein reporter to report on dynamic changes in redox environment (Figure 14A).(786) HEK293T cells loaded with RF1 (5 $\mu \mathrm{M}, 30 \mathrm{~min}$ ) showed increased fluorescence upon $\mathrm{H}_{2} \mathrm{O}_{2}$ stimulation $(100 \mu \mathrm{M}, 9 \mathrm{~min})$. The signal decreases after a further $6 \mathrm{~min}$ presumably due to restoration of cellular redox homeostasis. Addition of more $\mathrm{H}_{2} \mathrm{O}_{2}(100$ 
$\mu \mathrm{M}, 9 \mathrm{~min}$ ) can restore fluorescence signal. Despite significant progress, the use of fluorescent probes to report on reversible reduction-oxidation events in cells has been relatively limited compared to genetically-encoded sensors, the subject of the next section.

5.1.4 Genetically-encoded protein-based ROS sensors-One of the earliest examples of genetically-encoded sensors used a mutated form of YFP, $\operatorname{rxYFP}^{149202}$, in which redox-sensitive cysteines are introduced at the indicated positions.(805) Later, a circularly-permuted YFP (cpYFP) was discovered to respond to redox changes in the cell and was selective to $\mathrm{O}_{2}{ }^{--}$over other cellular oxidants.(806) Specifically, the study claimed to observe 'superoxide flashes' in mitochondria of live cells. The superoxide flashes were coupled to transient opening and closing of the mitochondrial permeability transition pore (mPTP), indicating that this process may be responsible for the superoxide burst. Subsequent studies by independent groups demonstrated that these " $\mathrm{O}_{2}{ }^{--}$bursts" are actually not caused by superoxide changes, but by transient changes in $\mathrm{pH}$ in the mitochondrial matrix.(807) The resting mitochondrial $\mathrm{pH}$ is 7.9.(808) However, changes in mitochondrial proton gradient during oxidative phosphorylation result in brief fluctuations in mitochondrial $\mathrm{pH}$. cpYFP has a $\mathrm{p} K_{\mathrm{a}}$ of $\sim 8.7$ and the fluorescent properties are acutely sensitive to changes in $\mathrm{pH}$, especially when the $\mathrm{pH}$ is within $1 \mathrm{p} K_{\mathrm{a}}$ unit of the sensor. The sensitivity of cpYFP towards $\mathrm{pH}$ is credited to structural changes introduced by the circular permutation, which exposes a pH-sensitive phenoxy group of the chromophore.(809)

Similar to rxYFP, another method of detecting redox changes in cells utilizes the idea of introducing redox-active cysteines into GFP. roGFP1 contains two cysteines introduced in place of S147 and Q204, and a C48S mutation to wt-GFP.(810) An additional S65T mutation of roGFP1 has yielded roGFP2 with brighter fluorescence and higher dynamic range compared to its parent sensor (9.2 fold for roGFP2 vs. 2.6 fold for roGFP1 at excitation wavelength of $488 \mathrm{~nm}$ ).(811) roGFP1, however, has a higher dynamic range in 405 excitation.(812) Hence, roGFP1 $\left(\mathrm{E}^{\circ}-291 \mathrm{mV}\right)$ may be more useful than roGFP2 $\left(\mathrm{E}^{\circ}\right.$ $-280 \mathrm{mV}$ ) in measuring redox changes in cellular compartment with higher reduction potential such as the cytosol and mitochondria.(812) Unlike cpYFP, the chromophore in roGFP is concealed.(813) Hence roGFP is relatively resistant to fluxes in pH. roGFP, however, equilibrates with the 2GSH/GSSG redox couple in cells and subcellular compartments and thus has limited utility in detecting specific ROS chemotypes. Instead roGFP-based reporters are ideal for measuring steady state glutathione redox potential in distinct subcellular compartments and overall redox changes induced by glutathione depletion.(812) More recently, a chimeric fusion of roGFP with Grx 1 has been developed that shows faster equilibration kinetics with cellular glutathione redox couple compared to roGFP probes.(812)

Recent advances have used clever techniques to make roGFP sense specific ROS chemotypes (Table 4). Genetic fusion of Orp1, a peroxidase from yeast, to roGFP2 has generated a sensor specific to $\mathrm{H}_{2} \mathrm{O}_{2}$ (Figure 16A).(814) The peroxidase Orp1 acts as the sensor for $\mathrm{H}_{2} \mathrm{O}_{2}$ and gets oxidized upon $\mathrm{H}_{2} \mathrm{O}_{2}$ encounter. This redox relay is then passed onto roGFP2 which results in the formation of a disulfide bridge between C147 and C204 and a consequent change in fluorescence of the sensor protein. Another recent method used Tsa2, a 2-cysteine peroxiredoxin from yeast, fused to a roGFP2 to generate an ultrasensitive 
(low micromolar detection limit) $\mathrm{H}_{2} \mathrm{O}_{2}$ sensor.(815) Peroxiredoxins are the most efficient thiol peroxidases with a near-diffusion-limited second-order rate constant for reaction with peroxide, $10^{8} \mathrm{M}^{-1} \mathrm{~s}^{-1}$,(111) a value almost two orders of magnitude higher than that with Orp1. Additionally, under non-stimulated conditions, ro-GFP2-Tsa $2 \Delta \mathrm{C}_{\mathrm{R}}$ is $\sim 50 \%$ oxidized thereby enabling measurement of $\mathrm{H}_{2} \mathrm{O}_{2}$ increase and decrease in cells.

A more recent addition to the arsenal of genetically-encoded sensors, called "HyPer", consists of a cpYFP inserted between residues 205 and 206 of a $\mathrm{H}_{2} \mathrm{O}_{2}$-sensitive OxyR regulatory domain (OxyR RD) from E. coli (816) (Figure 16A). Two cysteine residues, $\mathrm{C} 199$ and $\mathrm{C} 208$, in the OxyR RD are critical for its ability to sense $\mathrm{H}_{2} \mathrm{O}_{2}$. The peroxidatic C199, once oxidized to a sulfenic acid, forms a disulfide bond with the resolving C208 within OxyR RD. This disulfide bond formation is coupled to a large conformational change and an increase in fluorescence of the cpYFP. C199 has a low $\mathrm{p} K_{\mathrm{a}}$ in part because this residue is positioned next to a positively charged residue (arginine) which significantly enhances the cysteine's ability to react with $\mathrm{H}_{2} \mathrm{O}_{2}$. The rate constant for this reaction is in the order of $10^{5}-10^{7} \mathrm{M}^{-1} \mathrm{~s}^{-1}$. (817) In vitro, HyPer can detect as low as $25 \mathrm{nM} \mathrm{H}_{2} \mathrm{O}_{2}$. (817) Additionally, the topology around $\mathrm{C} 199$ is hydrophobic, likely favoring access of uncharged molecules to this critical residue. Thus, HyPer successfully discriminates $\mathrm{H}_{2} \mathrm{O}_{2}$ from $\mathrm{O}_{2}{ }^{--}$, oxidized glutathione, $\mathrm{ONOO}^{-}$and ${ }^{\circ} \mathrm{NO} .(818)$ Successive upgrades to HyPer have resulted in HyPer2(819) and HyPer3.(820) HyPer2 and HyPer3 show 3-fold higher dynamic range than HyPer. All probes show excellent kinetics of reaction with $\mathrm{H}_{2} \mathrm{O}_{2}\left(>10^{5} \mathrm{M}^{-1} \mathrm{~s}^{-1}\right)$. HyPer3 has a modestly improved dynamic range compared to HyPer (2.5 fold vs 1.7 fold) and was used for in vivo imaging of $\mathrm{H}_{2} \mathrm{O}_{2}$ gradient in larval zebrafish.(820) Since HyPer uses a circularlypermuted YFP, it is sensitive to fluctuations in $\mathrm{pH}$. Hence, $\mathrm{pH}$-specific probes must be used to monitor changes in $\mathrm{pH}$. The $\mathrm{H}_{2} \mathrm{O}_{2}$-insensitive $\mathrm{C} 199 \mathrm{~S}$ mutant of the sensor (SypHer) can serve this purpose.

One major advantage of HyPer and roGFP2-based $\mathrm{H}_{2} \mathrm{O}_{2}$ sensors is that they are ratiometric sensors. HyPer has two excitation maxima at $420 \mathrm{~nm}$ and $500 \mathrm{~nm}$ corresponding to the protonated and anionic form of YFP respectively, and one emission peak at $516 \mathrm{~nm} .(817)$ Oxidation of HyPer by $\mathrm{H}_{2} \mathrm{O}_{2}$ results in a decrease in $420 \mathrm{~nm}$ excitation peak and a proportional increase in the $500 \mathrm{~nm}$ excitation peak, permitting a ratiometric readout.(818) roGFP2-based sensors also have similar spectroscopic properties to HyPer (excitation maxima at $405 \mathrm{~nm}$ (reduced) and $488 \mathrm{~nm}$ (oxidized) and emission maximum at $510 \mathrm{~nm}$ ). (814) Both methods have several additional benefits over most small molecule probes. Firstly, they are reversible sensors and allow real time measurements of ROS changes in cells. A second benefit is that they can be directed to specific subcellular compartments simply by encoding protein-targeting sequences in the gene. $(386,481)$ HyPer has been used extensively both in cells $(818,821,822)$ and in whole organisms $(242,820,823)$ to measure $\mathrm{H}_{2} \mathrm{O}_{2}$ flux in real time.(817) A HyPer-EGFR fusion has allowed imaging of localized $\mathrm{H}_{2} \mathrm{O}_{2}$ generation at plasma membrane upon stimulation of platelet derived growth factor receptor (PDGFR) and endosomal $\mathrm{H}_{2} \mathrm{O}_{2}$ generation upon EGFR stimulation.(386) In vivo, the use of HyPer has shown the importance of $\mathrm{H}_{2} \mathrm{O}_{2}$ signaling in wound recognition,(824) neutrophil migration,(243) and wound healing in zebrafish embryos.(242) Similar experiments in Xenopus have elucidated the significance of $\mathrm{H}_{2} \mathrm{O}_{2}$ in tail fin regeneration after wounding. (823) A recently developed red fluorescent sensor of $\mathrm{H}_{2} \mathrm{O}_{2}$ that uses a circularly permuted 
mApple (cpApple) fused to OxyR-RD, termed HyPerRed,(825) is a welcome addition to obviate the naturally high background green fluorescence of biological systems (from flavins, NAD, FAs, etc.).(826,827) One interesting application of the varying palette of redox sensors is simultaneous sensing. The use of HyPerRed-mito (an intensity based ROS sensor that functions in the mitochondria) and Grx1-roGFP2-mito (a sensor of reduced glutathione, functioning in the mitochondria) showed that thapsigargin (an inhibitor of the sarco/endoplasmic reticulum calcium ATPase) only increases ROS levels in the mitochondria, while glutathione levels appear normal.(825)

Despite their advantages, the development of genetically-encoded sensors has been mainly limited to detecting $\mathrm{H}_{2} \mathrm{O}_{2}$. A similar approach to the aforementioned genetically-encoded sensors was undertaken to develop probes for visualization of organic hydroperoxides (OHP) in living cells.(828) OhSer, utilizes a circularly-permutated yellow fluorescent protein (cpVenus) fused to $\mathrm{X}_{\mathrm{c}}-\mathrm{OhrR}$, an OHP sensor and a transcriptional regulator of bacterial OHP detoxification genes. OhSer exhibited a 2-8-fold higher sensitivity to various OHPs over other ROS such as $\mathrm{O}_{2}{ }^{-}, \mathrm{H}_{2} \mathrm{O}_{2}$ and $\mathrm{HOCl}$ in vitro and showed a selective increase in fluorescence intensity following treatment of cells with OHPs. However, given the limited level of discrimination across varied ROS and OHPs, OhSer is unlikely to prove useful in detection of endogenously generated levels of OHPs.

\subsubsection{Electron paramagnetic resonance (EPR) methods to detect radical} generation in cells/organelles-EPR has been widely used to detect endogenouslygenerated radical species of relevance in redox signaling. $(831,832)$ The underlying principle of EPR is similar to nuclear magnetic resonance (NMR): an externally-applied magnetic field is applied to unpaired electrons (contrasting NMR where nuclei are excited) and energy emitted upon relaxation to the ground state is measured. Thus, molecules with paired electrons (the bulk of biological samples) are not detected by EPR, rendering EPR very sensitive for radical species and paramagnetic metal complexes, amongst other paramagnetic biological molecules. Furthermore, EPR can discriminate between different radical species. This is because each element provides a specific local environment to an electron that affects how energy levels differentiate in a specific magnetic field. Numerically this value is defined by the Lande g-factor $\left(g_{e}\right)$. Additionally, "EPR hyperfine coupling" analogous to $J$-coupling in NMR and resulting from interaction of the magnetic moment of the nuclei of the radical species and that of the unpaired electron provides characteristic spectra for individual radical species. EPR techniques are also highly sensitive and quantitative. Detection of $\mathrm{O}_{2}{ }^{-\boldsymbol{}}$ by EPR spin trap method was shown to be 40 times more sensitive than a commonly used spectrophotometric technique involving reduction of ferricytochrome C.(833) Also, the direct quantitation of the amount of redox-active radical present in the sample is facile through the evaluation of EPR signal intensity against appropriate control samples of known concentrations.

A point of consideration is that many of the endogenously-relevant redox signals are highly reactive and short-lived (Table 1). In fact, the concentration of free radicals can be several to hundreds of fold below the EPR detection limit. Hence EPR techniques for successful detection of these species almost always require a method to trap the radicals using EPR 'silent' probes. These so called "spin traps" claim sensitivity down to nanomolar levels in 
some cases.(834) The most common of such probes are cyclic nitrones such as 5,5Dimethyl-1-Pyrroline-N-Oxide (DMPO), 5-Boc-5-methyl-1-Pyrroline-N-Oxide (BMPO), and 5-(Diethoxy phosphoryl)-5-methyl-1-pyrroline-N-oxide (DEPMPO) (Figure 16B). $(831,835,836)$ To detect a short-lived radical species, an EPR-silent trap is first introduced into the system. The trap reacts with the transient redox radical through adduction to the $\mathrm{C}-$ $\mathrm{N}$ bond. The resultant dative stabilized radical, ${ }^{\circ} \mathrm{N}-\mathrm{O}$, is then detected by EPR (Figure 16B, Inset). Therefore, this method is not particularly dynamic, and also quenches the biological radical, likely inhibiting signaling pathways. Importantly, some spin traps affect signaling pathways, including Erk,(837) AR and apoptosis.(838) Another major problem with the use of EPR to detect radical generation in vivo is that the commonly used spin-traps are nondiscriminatory against endogenously-derived radicals. For example, DMPO and DEPMPO react with $\mathrm{O}_{2}{ }^{-}$and ${ }^{\circ} \mathrm{OH}$ to generate ${ }^{-} \mathrm{OOH}$ and ${ }^{-} \mathrm{OH}$ adducts respectively. The rate constant for the reaction of DMPO with $\mathrm{O}_{2}{ }^{-}$is in the order of $50-60 \mathrm{M}^{-1} \mathrm{~s}^{-1}$; this reaction generates a stable intermediate ${ }^{\circ} \mathrm{N}-\mathrm{O}$ radical containing a metastable peroxide which has a relatively short half-life $\mathrm{pH}$ 7. Comparatively, ${ }^{\circ} \mathrm{OH}$ reacts with DMPO at the diffusion limit to generate an intermediate alcohol bearing a ${ }^{\circ} \mathrm{N}-\mathrm{O}$ with a half-life of 50 min at $\mathrm{pH}$ 7.(836)

One drawback of using spin traps is that the EPR spectrum of the trapped radical is independent of the radical detected. Significant effort has been expended to develop spin traps that are specific to a particular ROS. For instance, a series of superoxide selective spin traps have been developed: the mitochondria-targetable spin trap, Mito-DIPPMPO (bearing a triphenyl phosphonium motif)(839) and the extracellular-targeted trap, CD-DMPO [housing a permethylated $\beta$-cyclodextrin $(840,841)$ ]. These probes show 3-fold reactivity enhancement for superoxide over DEPMPO and BMPO.(841) Mito-DIPPMPO and CDDMPO both detect $\mathrm{O}_{2}{ }^{--}$generated in PMA-stimulated RAW246.7 cells, with no background signal arising from ${ }^{\circ} \mathrm{OH} .(839,842)$ The probes are sensitive enough to also detect basal $\mathrm{O}_{2}{ }^{\text {- }}$ generation in unstimulated macrophages.(843) The ability of these probes to selectively detect $\mathrm{O}_{2}{ }^{--}$has been ascribed to the fact that the hydroxyl radical reacts with these probes with low chemoselectivity [reaction between tetraphenylphosphonium ion and hydroxyl radical for instance is close to diffusion control(844)] and thus does not give an appreciable amount of the dative stabilized ${ }^{\circ} \mathrm{N}-\mathrm{O}$ that is the more favored product of reaction with $\mathrm{O}_{2}{ }^{\bullet-}$.

a-(4-Pyridyl $N$-oxide)- $N$-tert-butylnitrone, POBN, another nitrone-based spin trap, and DMPO have been used to study lipid-derived radical generation by oxidation of PUFAs (Figure 16B).(845,846) POBN is ideal for detection of carbon-based radical such as those generated during lipid peroxidation because of the long half-life of the trapped radical. Carbon-centered radical adducts with POBN have a lifetime of a few hours thus enabling efficient detection.(845) A major disadvantage of using POBN as a spin-trap, however, is that all carbon-based radical adducts with POBN have similar spectra making discrimination of individual carbon-based radical species from PUFA oxidation impossible. Additionally, POBN adducts resulting from reaction with oxygen-based radicals, such as lipid peroxy radical intermediates in PUFA oxidation, have very short half-lives $\left(t_{1 / 2}<10 \mathrm{~s}\right)$, thereby limiting their use for detecting oxygen-based radicals.(845) DMPO and EMPO, on the other hand, have proven useful in extending utility to trapping oxygen radicals. The half-life of peroxy and alkoxy adducts of EMPO are on the order of 10 minutes or $>1 \mathrm{~h}$, respectively. 
(847) Recently, an assortment of spin traps has emerged which detects a larger repertoire of redox-signal chemotyopes, including sulfur-based and nitrogen-based radicals.(848)

Spin-traps can be expensive and have other downsides when used for biological studies such as permeability and cytotoxicity. Additionally, real life biological systems are very complex, so deconvolution of spectra, a common practice in in vitro experiments, is much harder. Finally, in the case of pyrroline-N-oxide probes numerous processes can lead to ${ }^{\circ} \mathrm{N}-\mathrm{O}$ products that are EPR active, but are not actually derived through radical attack on the $\mathrm{C}-\mathrm{N}$ bond.(849) Two principal off pathway processes leading to EPR-active products are inverted spin trapping(850) and Forrester-Hepburn reaction(851) (considered to be the most common off pathway mechanism). Nevertheless, spin-trap EPR methods have been used for detecting radical generation in cells, $(835,852)$ and have shown promise in multiple in vivo studies. (853) Novel methods have focused on generating subcellular domain-localized spin-traps which will allow measurement of localized redox signal generation. In this regard, although some success has been achieved as outlined above, the probes still have poor reaction kinetics.(854) Moreover, hydrophobic probes/traps that allow measurement of lipid peroxides in membrane are also underdeveloped. Steps to develop spin traps with different lipophilicities that can be used in the same samples to develop a multidimensional probe for specific free radicals have been disclosed.(848) However, it seems that significant work will be required to extend this regimen into a general approach for radical deconvolution. An additional problem with EPR-based approaches is their inability to detect and measure generation of non-radical redox species such as $\mathrm{H}_{2} \mathrm{O}_{2}$ and $\mathrm{ONOO}^{-}$. Recent methods have focused on finding novel EPR spin traps that convert these non-radical redox signals to EPRactive radical signal upon reaction. $\mathrm{CP}-\mathrm{H}$, for example, has been used to trap $\mathrm{ONOO}^{-}$that generates a stable nitroxide radical upon reaction.(855) $\mathrm{CP}-\mathrm{H}$, however, can also react with $\mathrm{O}_{2}{ }^{--}$thus complicating the analysis. An alternative to EPR studies is immuno-spin trapping wherein a stable spin trap reacts with protein radicals to form covalent protein strap adducts that can be detected immunologically.(856)

5.1.6 Small-molecule fluorescent probes to detect free RES-Formaldehyde is present in the brain at concentrations around $100-400 \mu \mathrm{M} .(857,858)$ Because of its reactivity, ability to form Schiff bases readily and the fact that those Schiff bases undergo chemistry readily, chemoselective probes for formaldehyde have been developed. FAP-1 is an example of such a sensor (Figure 16C).(859) This molecule can form a Schiff base with formaldehyde and after an aza-Cope rearrangement and hydrolysis give a fluorescent dye (a process leading to an 8 -fold increase in fluorescence). Using this probe, exogenous formaldehyde in the $100 \mu \mathrm{M}$ range was detected in cells. This probe was chemoselective for formaldehyde over various aldehydes (including acetaldehyde and HNE), although it is likely that the probe is selective for formaldehyde over many different aldehydes and ketones as imine formation for acetals and ketones is slower than for formaldehyde, and the cyclohexane-like Cope transition state is severely crowded. Similar formaldehyde-selective probes have also been reported by other labs (Figure 16C).(860)

Coupling a promiscuous sensing reaction (e.g. Schiff base formation) to a secondary reaction that only one type of reagent can do efficiently (e.g. 3,3 shift) is a good general mechanism to design a specific probe. A similar strategy developed by the Spiegel lab is 
their "turn-on" fluorescence sensor of methylglyoxal (Figure 16C).(861) Using a 1,2diamino functionalized BODIPY probe, allowed fluorescence turn-on only by 1,2dicarbonyl compounds [of which by far the most abundant in cells is methylglyoxal(443)].

Other detection methods of formaldehyde have used a hydrazine conjugated to a fluorophore. $(862,863)$ In the native state, the free amine on the hydrazine quenches fluorescence of the conjugated fluorophore through PET. Upon reaction with the aldehyde, the hydrazine forms a formimine, which blocks PET, thus 'turning-on' fluorescence.

Similar methods using hydrazine conjugated fluorophores have also been used to detect other reactive aldehydes. Probes have been developed wherein a molecule containing a dye and a quencher in tandem is split to a free dye upon exposure to a generic carbonylcontaining compounds.(864) This strategy can give good signal to noise (again when cells treated with the molecules are exposed to exogenous carbonyl compound). One drawback of this method is that a catalyst is required to drive fragmentation of the self-quenching molecule. In general, such two-component systems make it particularly difficult to design good controls, and hence can affect confidence in the results. Finally, a similar sensor of malondialdehyde has been reported. In this set up, a pendant hydrazine moiety quenches a dye to which it is attached through PET transfer (Figure 16C).(865) When hydrazine reacts with malondialdehyde, PET is reduced, and fluorescence is activated.

\subsection{Identifying targets of RES/ROS modification}

Redox signaling selectivity is almost exclusively controlled by kinetic adduction rates. Of the canonical protein side-chains, cysteine is the most nucleophilic and hence the more sensitive amino acid residue to ROS/RES-mediated modification. Not surprisingly, there have been considerable efforts to develop methods to detect oxidative and electrophilic modifications of cysteine. Recently, proteomics approaches have gained wide-spread usage because of their versatility and generality. Mass Spectrometry approaches can identify redox-sensitive targets in lysate, cells, tissues and whole organisms. MS approaches can be either direct or indirect.

5.2.1 Indirect approaches to detect oxidative modifications-Indirect approaches to detect cysteine modification rely on the loss of reactivity of the modified cysteine residues with thio-alkylating probes (Figure 17A) or the regain of reactivity of the modified cysteine after treatment with reducing agents (Figure 17B). Probes can be functionalized with a fluorophore for gel-based fluorescence analysis. Biotinylated or alkyne-functionalized probes are the probes-of-choice for enrichment for western blot or MS analyses. For many RES, such as HNE, alkyne modification is an almost ideal modification as it is sterically non-intrusive and biorthogonal. Under click conditions, the alkyne can be converted to the appropriate fluorophore/affinity handle, post conjugation.

5.2.1.1 Detecting sulfenic acid modification: With growing evidence of cysteine sulfenylation modulating biological processes, $(5,484,866-868)$ there is an increasing interest in identification of proteins prone to sulfenic acid modification in cellular and organismal context. This modification can be detected using a tagged alkylating agent such as a biotinconjugated iodoacetamide (BIAM) to label "all nascent thiols" in the proteome. Oxidized 
cysteines show reduced reactivity toward the probe and thus reduced signal intensity on a biotin(avidin) blot. The loss of labeling corresponds to an increase in oxidation of cysteine thiols (Figure 17A). A modified version of this approach allows detection of oxidized proteins as increase in signal intensity rather than a decrease (Figure 17B). This method is based on biotin switch technology (BST) initially developed to detect -SNO modification of cysteine residues.(869) This indirect method involves (1) treatment with an alkylating agent such as N-ethyl maleimide (NEM) or iodoacetamide (IAM) to 'cap' "all” free cysteines; (2) reduction of oxidized cysteines by treatment with a reducing agent; and (3) labeling of nascent thiols with BIAM and subsequent gel-based analysis or MS approach to detect oxidized proteins. Oxidized proteins show enhanced signal in biotin(avidin) blot or through in-gel fluorescence (Figure 17B). However, these methods are qualitative and in addition do not provide any information on the nature of oxidized cysteine. In fact, any kind of cysteine modification results in a decrease in signal, which may amplify the number of 'falsepositives'. Some selectivity in detecting these signals has been achieved using different reducing agents. Arsenite, for example, selectively reduces $S 4$ sulfenylated proteins.(870) DTT, on the other hand, reverses all oxidative modifications on cysteines except sufinic and sulfonic acid modifications.

Additional variations using isotope labeled reagents have enabled quantitative assessment of protein thiol oxidation. A BIAM-based technique uses acid-cleavable isotope-coded affinity tags [ICAT; a molecule consisting of biotin, an isotope (typically ${ }^{13} \mathrm{C}$ )-labeled 9-carbon linker region, and an iodoacetamide function] to detect and simultaneously quantify redoxsensitive protein thiols.(871) In this method, free thiols of an untreated/control sample are labeled with a light ICAT reagent $\left({ }^{12} \mathrm{C}\right.$-ICAT) by treating the soluble proteome. Lysate from a parallel sample subjected to an oxidant is treated with a 9-Da heavier ICAT reagent $\left({ }^{13} \mathrm{C}\right.$ ICAT). The heavy and light ICAT reagent labeled lysates are then mixed, trypsinized, and enriched using streptavidin. Eluted peptides are analyzed using LC-MS and the ratio of heavy-to-light peptide is quantitated. Because oxidized cysteines display lower reactivity towards the BIAM-based probe, peptides prone to oxidation have reduced signal intensity in the heavy ${ }^{13} \mathrm{C}$-ICAT reagent-treated sample. Importantly, because the heavy and light ICAT reagents only differ by a molecular weight of $9 \mathrm{Da}$, MS fragments show two peaks separated by $9 \mathrm{Da}$ that can be quantitatively analyzed.(872,873) A variation of BST using ICAT reagents has enabled quantitative detection of reversibly-modified thiols. In this method, free thiols in soluble lysate are first alkylated. Next, reversibly-modified thiols are reduced and labeled with either light or heavy ICAT reagents and the samples are analyzed as described above.(874,875) ICAT-based BST has also been used to differentially label oxidized and reduced cysteines within the same sample, in a method termed oxICAT.(876) In this method, the soluble proteome from cells is first treated with a light ${ }^{12} \mathrm{C} 2$ ICAT to label any free cysteines. The reversibly-oxidized cysteines in the same sample are then reduced and subsequently modified with heavy ${ }^{13} \mathrm{C}$-ICAT reagent. This method avoids problems associated with differences in protein expression between two samples. oxICAT method has been used to quantitatively assess cysteine oxidation upon oxidative stress in cells. $(876,877)$

5.2.1.2 Profiling cysteine modification by LDEs using ABPP: Activity-based protein profiling (ABPP) has emerged as a powerful tool to rank the activities of selected proteins in 
complex proteomes. Early examples of ABPP focused on the use of active-site directed chemical probes to profile cysteine proteases(878) and serine hydrolases.(879) MS coupled with ABPP — dubbed tandem orthogonal proteolysis (TOP)-ABPP — has allowed for simultaneous detection (albeit indirect using proxy probes similar to those used above) of protein targets and (with several assumptions) the sites modified by chemical probes. $(880,881)$ Using TOP-ABPP it was shown that different classes of electrophiles exhibit distinct labeling profiles in a "soluble mouse proteome" (lysate). Chloroacetamide and a, $\beta$ unsaturated ketone probes showed selectivity towards cysteine, whereas phenylsulfonate esters also targeted aspartate, glutamate, tyrosine and histidine residues in addition to cysteines.(880) More importantly, all electrophiles showed reactivity toward functional residues in enzymes despite the large excess of non-functional residues in the proteome, suggesting that functional residues display enhanced nucleophilicity. ABPP using isotopelabeled tags, called isoTOP-ABPP, was recently used to quantitatively profile 'hyper reactive' cysteines in the proteome.(881) isoTOP-ABPP uses a broadly reactive probe such as iodoacetamide that covalently modifies (some) reactive cysteines. $(882,883)$ Typically, a soluble lysate is first treated with different concentrations of the iodoacetamide probe. An alkyne handle on the probe allows for click coupling with either heavy or light isotopelabeled cleavable tags. Subsequent enrichment of probe-modified reactive cysteines and mass spectrometry allows quantitative comparison of the reactivities of the cysteine residue towards the electrophilic probe. Modification of the ABPP method using a competitive electrophilic probe, called competitive isoTOP-ABPP, has allowed identification of LDE6 sensitive sites in the proteomes.(884) In this method, a cell/soluble proteome is first treated with a competitive LDEs. The control set is treated with the vehicle (e.g. DMSO) (Figure 17C). Subsequently, both sets of cells are lysed independently and each lysate is labeled with alkyne-functionalized iodoacetamide. Click coupling of isotope-labeled tags and subsequent MS analysis allows for the quantitation of cysteines that are sensitive to LDE modification. A decrease in ratio of cysteine-containing peptide in the LDE-treated cells compared to untreated samples identifies modified cysteine residues. Competitive isoTOPABPP was used to quantitatively profile the reactivities of $>1000$ proteins to three representative electrophiles HNE, 2-trans-hexadecenal (2-HD) and 15d-PGJ 2 .(884) In addition to profile the reactivities of electrophiles, the isoTOP-ABPP method has been used to monitor S-nitrosation of proteins upon treatment with S-nitrosoglutathione, (885) and cysteine oxidation upon exogenous treatment of pathogenic bacteria to $\mathrm{H}_{2} \mathrm{O}_{2}(886)$ and EGF stimulation of mammalian cells.(887)

5.2.1.3 ReACT strategy to profile methionine oxidation: A new method to identify reactive methionines (dubbed ReACT) was recently disclosed.(888) ReACT uses an oxaziridine-based small-molecule probe that was optimized to afford products of methionine nucleophilic addition to nitrogen as opposed to oxygen. By functionalizing the amine within the oxaziridine with an alkyne (or an azide motif), modified proteins were identified by biotin Click/streptavidin pulldown followed by digest MS. By varying the amount of oxaziridine added to the lysate, and choosing proteins that react only with low concentrations of oxaziridine, a hyper-reactive methionine (Met169) was identified in human enolase. The corresponding methionine was subsequently implicated in the response of yeast to hypochlorite. 
This work represents a pioneering first step in methionine reactivity profiling, adding to the growing suite of reactive electrophilic probes for proteome reactivity mapping. To this end, further considerations worth addressing include the extent to which the synthetic electrophile mimics native electrophiles targeting the protein residue (in this case, oxaziridine in driving bond forming events with protein-methionine), compatibility/ permeability of the probe with living cells/intact specimens (beyond lysates), and selectivity/ non-invasiveness of the probe to modifications via oxaziridine oxygen (beyond nucleophilic addition to oxaziridine nitrogen as analyzed in this key inaugural example with methionine) since oxaziridine oxygen may be a chemically more favored route in the case of amine and thiolate nucleophiles.

5.2.1.4 General limitations of indirect methods: Indirect approaches to profile redoxmodified proteins are invaluable tools. However, they suffer from several important drawbacks that mean data must be interpreted with caution. Most importantly, indirect methods are limited by the scope of residues covered by the proxy. For instance, in cysteine ABPP, a fraction (around 1\%) of all cellular cysteines are detected. Thus, a high-resolution picture of a fraction of the cysteome is painted, while the bulk of cysteines are ignored. Additional issues include: as these systems are typically assayed in lysates (soluble proteome) exposed to a bolus of reagents, they are unable to capture spatiotemporal nuances of redox signaling. Secondly, there is no precise information provided on what mechanism leads to loss of the proxy: the profiled residue could possibly be modified in several ways other than by the compound of interest including oxidation, degradation, change in secondary associations and disulfide bond formation. Additionally, using a proxy such as IAM or NEM has its intrinsic limitations. Any protein that does not react with the proxy probe, regardless of whether it reacts with the compound of interest, cannot be scored as a hit. Additionally, even though these proxies are thiol-reactive, they have different reaction chemistries, transition state addition geometries and give different products, all of which influence the product spectrum. $(889,890)$ For instance, IAM reacts with thiols using $\mathrm{S}_{\mathrm{N}} 2$ chemistry whereas NEM reacts through Michael addition. NEM and IAM also have different reaction kinetics with NEM being at least 2 times more reactive than IAM to thiols under physiological conditions.(889) The rate of reaction between NEM and cysteine is also much less $\mathrm{pH}$ dependent than reaction of cysteine with IAM. NEM also reacts with lysine and histidine side chains in addition to cysteine which may influence profiling results (either through direct addition, or indirectly through influencing reactivity of other residues/ associations/stabilities) and should be considered when choosing the right proxy. $(889,891)$ Both NEM and IAM react with sulfenic acids, which may affect quantitative analysis by underestimating sulfenic acid content,(892) although with careful analysis such adducts can be differentiated from adducts to thiols by mass spectrometry. Finally, across the iterations of general profiling reagents developed (IAM to oxaziridine), the complexity of the probes has increased. It has not been systematically addressed how the specific residues labeled by the probes is biased by the "ligandable" interaction of the probes. However, such considerations should really be addressed (possibly by adding an inert analog to the mixture and measuring which residues show reduced labeling) prior to declaring that a general probe has been developed for profiling a specific residue. 
In addition, as indirect methods measure loss of labeling, they are prone to false positives: if modification of a functionally-coupled residue to a cysteine hinders its ability to label the probe and therefore its detection, it may show up as a positive hit. Independent secondary validation can mitigate such problems, although reduces the overall utility of the method. Additionally, some indirect approaches such as competitive-isoTOP ABPP require treatment with the probe in lysate because of probe impermeability and/or toxicity. $(881,884,893)$ This strategy is ultimately flawed since different cellular components are at different redox states(187) and the oxidation state of the cysteine residues may be affected giving confounding results. Moreover, uncontrolled bulk exposure of cells and organisms to reactive small-molecule signals may result in global modification of proteins that may not be physiologically relevant. Recently developed caged electrophilic proxy probes, which are cell-permeable and release the reactive probe upon UV light illumination, mitigate the issue of high toxicity and cell permeability encountered with proxies such as IAM. $(887,894)$ Additionally, performing isoTOP ABPP on isolated intact organelles such as mitochondria may help preserve organelle redox environment,(895) although such protocols are complex and the benefit relative to performing experiments in live cells/organisms is likely marginal. Finally, this method will also likely not be able to identify residues that are endogenously modified as they may not be detected by the probe.

\subsubsection{Direct methods to detect cysteine modifications}

5.2.2.1 Nucleophilic probes for detecting sulfenic acid modification: Small-molecule probes for direct detection of sulfenic acid modification exploit the ambiphilic nature of the modification. Most recent advances in detecting sulfenic acid modification are based on the use of 5,5-dimethyl-1,3-cyclohexanedione (dimedone).(5,395,896-899) The earliest reaction between protein sulfenic acid and dimedone was used to characterize oxidized GAPDH. Covalent modification of GAPDH-SOH with dimedone inactivated the acyl phosphatase activity of oxidized enzyme.(900) Since then, many fluorescence-, $(896,901)$ biotin-, $(901,902)$ azido-, $(903,904)$ and alkyne(905)-tagged dimedone-based probes have been developed to detect sulfenic acid in vitro and in cells. Although dimedone is reactive towards aldehydes and amines at very basic $\mathrm{pH}$ or in organic solvents, no such reactivity was observed in aqueous conditions.(896,906) Additionally, dimedone is also reported to be selective towards sulfenic acid as other cysteine oxoforms such as $S$-nitrosothiol, sulfoxides, sulfinic and sulfonic acids, and disulfide are unreactive with the probe in aqueous conditions. However, sulfenylamides have been recently shown to react with dimedone.(907)

The first dimedone-based probe consisted of the fluorophores isatoic acid (DCP-MAB) or 7methoxycoumarin (DCP-MCC) conjugated to dimedone (Figure 18A).(896) The reactivity of the probes to sulfenic acid was tested using a mutant $\mathrm{AhpC}$, a 2-cysteine peroxidase enzyme, that forms a stable sulfenic acid because it lacks the resolving cysteine. The stabilized sulfenic acid was efficiently labeled with DCP-MAB and DCP-MCC. The flurry of second and later generation probes has now found widespread use. For example, DCPBio1 was used to detect sulfenylation of the protein tyrosine phosphatases SHP-1 and SHP-2 as well as actin upon T-cell activation.(908) Sulfenylation of the protein targets was critical for the downstream signaling responses in these cells. DCP-Bio1 was used to show that a specific kinase isoform is susceptible to sulfenylation. Akt2 was identified as sulfenylated 
upon growth factor stimulation of fibroblast cells.(868) Recent work using DCP-Bio1 has shown increased protein sulfenic acid modification upon VEGF stimulation of endothelial cells.(909) The authors demonstrated that in actively migrating HUVECs, VEGF-induced protein sulfenic acid colocalized with the cytosolic subunit of NOX and IQGAP1, an actinand Rac1-binding scaffolding protein, at the leading edge of the lamellipodia. Although the authors cede that this is certainly not direct evidence that IQGAP1-SOH is involved in migration, suppression of global S-OH using $\mathrm{N}$-acetyl cysteine or dimedone, reduced migration of cells.

More recent additions to the arsenal of functionalized probes include alkyne and azidotagged dimedone (Figure 18A). $(903,905,910)$ Compared to probes with bulky tags such as biotin and fluorescent molecules, azido and alkyne-functionalized probes manifest better cell permeability. Another important consideration is that bulky tags may compromise protein labeling efficiency. DAz-1 and DAz-2, two azido-functionalized dimedone probes, and DYn-1 and DYn-2, two alkyne-functionalized probes, were recently developed. DAz-1 shows reduced reactivity with sulfenic acid compared to dimedone;(903) this issue was solved by development of DAz-2.(910) DAz-1 has been used to detect the global increase in sulfenic acid modification upon treatment of Jurkat cells with hydroperoxides.(904) Interestingly but perhaps unsurprisingly, distinct protein labeling patterns were observed when the probe was treated in lysate or in cells, underscoring the importance of probing these transient modifications in vivo (provided the probe has not biased localization, this would imply some locales respond stronger/ to a greater extent of sulfenic acid protein modifications following bolus exposure to $\mathrm{OOH})$. DAz-2 has also been used to profile sulfenic acid modification in cells.(910) HeLa cells in suspension were treated with DAz-2 for $2 \mathrm{~h}$ in low serum media; modified proteins were enriched using Staudinger ligation with phosphine-labeled biotin and subsequently purified using Neutravidin beads. Enriched proteins were analyzed using mass spectrometry following gel electrophoresis and in-gel trypsin digest. Using this protocol, the authors identified 193 proteins sulfenylated in HeLa cells. 14 of these proteins were previously characterized to be prone to sulfenic acid modification. The identified proteins have been implicated in several physiological processes including redox homeostasis, DNA damage repair, metabolism, etc.(910) Recently, using the alkyne-functionalized probe, DYn-2 (Figure 18A) increase in protein sulfenylation was observed upon treatment of A431 cells with EGF.(905) The authors also identified EGFR as a novel sulfenylated protein. Interestingly, sulfenic acid modification resulted in a 1.5-fold increase in kinase activity of the enzyme. ESI-LC-MS/MS identified C797 within the ATP binding site of the enzyme as the sensing residue. A follow-up study demonstrated that $\mathrm{C} 797$ on EGFR is solvent-exposed, has a $\mathrm{p} K_{\mathrm{a}}$ of $\sim 5.5$, and reacts with $\mathrm{H}_{2} \mathrm{O}_{2}$ with a second-order rate constant of $>110 \mathrm{M}^{-1} \mathrm{~s}^{-1}$, distinguishing this residue from the remaining five other cysteines.(911) Importantly, mutation of this sulfenic acid-sensitive cysteine residue to a serine or alanine residue showed diminished response to EGF- or $\mathrm{H}_{2} \mathrm{O}_{2}$-mediated activation of the enzyme. However, it is important to note that the $\mathrm{C} 797$ mutant kinases had 4-7-fold lower kinase activity than the WT protein. Nevertheless, computational modeling identified an arginine residue, R841, which can interact with sulfenylated C797 and stabilize the catalytic loop, thus enhancing the kinase activity of the sulfenylated enzyme.(911) 
Dimedone-based probes have also been used for quantitative determination of redoxdependent changes in sulfenic acid in purified proteins and in cells. One method used either native DAz-2 ([D0]-DAz-2) or deuterium-labeled DAz-2 ([D6]-DAz-2), to compare the relative oxidation of purified Gpx3 (a C64S/C82S mutant where two non-catalytic cysteines had been mutated was used) upon treatment with either low ( 0.1 equivalent) or high concentrations (1.5 equivalent) of $\mathrm{H}_{2} \mathrm{O}_{2}$ for $2 \mathrm{~h}$ in vitro.(912) $\mathrm{Gpx} 3$ stimulated with low concentration of $\mathrm{H}_{2} \mathrm{O}_{2}$ was conjugated with [D0]-DAz-2 and Gpx3 stimulated with high $\mathrm{H}_{2} \mathrm{O}_{2}$ concentration was conjugated with [D6]-DAz-2. The samples were then mixed 1:1, 'clicked' with alkyne-functionalized biotin with an acid cleavable linker, enriched and subsequently analyzed using mass spectrometry. The method successfully measured a 2-fold increase in oxidation of $\mathrm{Gpx} 3$ at the catalytic $\mathrm{C} 36$ upon treatment with higher concentration of $\mathrm{H}_{2} \mathrm{O}_{2}$. A similar method used an isotope-labeled [D6]-dimedone to first covalently modify sulfenic acid.(897) Subsequently, excess reagent was removed, and the sample was treated with iododimedone to modify any free thiols. The fraction of sulfenic acid modified protein could be determined by dividing the heavy-isotope labeled peak by the sum of the heavy and light peaks.(897) Another study using a light and heavy isotope9 labeled DYn-2 (DYn-2[D0] versus DYn-2-[D6]) recently identified $\sim 1000 S$-sulfenylation sites from $>700$ proteins in RKO cells treated with $500 \mu \mathrm{M} \mathrm{H}_{2} \mathrm{O}_{2}$ for $5 \mathrm{~min}$.(898) Additionally, the method was used to quantify the changes in global sulfenylation upon EGF stimulation of A431 cells.(913)

Despite the widespread use of dimedone-based probes, one of the major concerns has been its slow reactivity with sulfenic acid. $(906,914)$ Linear $\beta$-ketoesters, and derivatives of 1,3cyclopentadione(915) have modestly higher reactivity with sulfenic acid than dimedone. (916) For example, the pseudo-first-order rate for reaction of sulfenic acid with 1,3cyclopentadione is $0.0036 \mathrm{~min}^{-1}$ compared to $0.0024 \mathrm{~min}^{-1}$ for dimedone at $\mathrm{pH} 5.5$ at room temperature. A thorough screen of a library of cyclic carbon nucleophiles recently identified several classes of "C19 nucleophiles" that feature 100- to 200-fold enhanced reactivity to sulfenic acid compared to dimedone.(917) A follow-up screening of possible targets of these different classes of carbon-nucleophiles identified $>1280$ sulfenylated proteins in nonstimulated RKO soluble proteome.(918) Surprisingly, only nine proteins were labeled by all the nucleophiles, demonstrating differential reactivity preferences. An important consideration is that treatment with different probes may alter the redox state of cells differently, thereby yielding non-identical labeling. Trapping cysteine-SOH, particularly for long incubation times, may significantly alter redox dynamics of the cell. Additionally, even with the fastest dimedone-derived probes that attempt to quantitatively trap cysteine-SOH at sufficiently rapid rates, the desired sulfenic acid trapping may not be possible if the reaction kinetics favors formation of mixed disulfides with GSH or other proteins.

5.2.2.2 Electrophilic probes for detecting sulfenic acid modification: Relatively fewer electrophilic probes for cysteine-SOH exist. 7-Chloro-4-nitrobenzo-2-oxa-1,3-dizole (NBDCl) (Figure 18B) has been used to demonstrate sulfenylation of the peroxidatic cysteine in alkyl hydroperoxide reductase $\mathrm{C}$ (AhpC) during the catalytic cycle of the enzymes.(919) A mutant $\mathrm{AhpC}$ where the resolving cysteine was mutated to a serine was used to trap the sulfenic acid intermediate. Although NBD-Cl can react with thiols, amines, and phenols 
under physiological $\mathrm{pH}$, only thiol reactivity has been observed in vitro. NBD-Cl has also been used to characterize protein sulfenic acid formation in PTP,(920) human serum albumin,(921) and human a 1-antitrypsin(922) in vitro. Recently, strained cycloalkynes have been developed as probes for sulfenic acid with 100 times faster reaction kinetics than dimedone (Figure 18B).(914) However, caution must be exercised as strained cycloalkynes react spontaneously with numerous nucleophiles, including thiols.

5.2.2.3 Detecting sulfinic and sulfonic acid modifications: Despite the growing evidence of sulfinic/sulfonic acid modifications in redox regulation of proteins, $(683,684,696,697)$ there are only a few methods to detect such modifications. Sulfinic acid and sulfonic acid modifications on cysteines can be detected using mass spectrometry by a shift of $32 \mathrm{Da}$ and $48 \mathrm{Da}$, respectively in the molecular weight of the cysteine residue. $(923,924)$ However, this approach may be biased by adventitious oxidation during processing (especially in test samples that are already under oxidative stress) and it may also not have the resolution to distinguish constitutional isomers, e.g. hydropersulfides versus sulfinic acids. Coupling this approach with 2-D gel electrophoresis relying on the acidic shift caused by sulfinic and sulfonic modifications has also been used.(925,926) However, these methods are low throughput and have limited applications in profiling novel targets of cysteine sulfinic/ sulfonic acid modifications. Enrichment of modified proteins followed by digest mass spectrometry is an appealing option. Enrichment methods have focused on the use of the additional negative charge on $\mathrm{SO}_{3} \mathrm{H}$ at acidic $\mathrm{pH}$. The $\mathrm{p} K_{\mathrm{a}}$ of $\mathrm{SO}_{2} \mathrm{H}$ and $\mathrm{SO}_{3} \mathrm{H}$ are $\sim 2$ and $~$ -3 respectively.(927) Selection of $\mathrm{SO}_{3} \mathrm{H}$-modified peptides can be performed using either positively- or negatively-charged resins. $(924,928)$ Polyarginine-coated nanodiamonds can selectively enrich sulfonylated BSA and oxidized insulin chains A and B from a complex mixture of tryptic peptides.(924) The enrichment method showed excellent selectivity toward sulfonylated peptide such that oxidized insulin chain could be detected even in the presence of 5000-fold excess of contaminating peptides. Importantly, enrichment using polyarginine-coated nanodiamonds coupled with MALDI-TOF/TOF MS was also selective for sulfonylated peptides over poly-phosphorylated peptides, even though the two groups are chemically very similar. Recently, a tandem negative selection using strong cation exchange followed by a positive selection by Hydrophilic Interaction Liquid Chromatography (HILIC) identified $\sim 180 \mathrm{SO}_{2} \mathrm{H} / \mathrm{SO}_{3} \mathrm{H}$ modified cysteine sites in rat myocardial tissue extracts treated with $\mathrm{H}_{2} \mathrm{O}_{2}$.(929) Importantly, IR injury via Langerdorff perfusion, a method to study the heart as an isolated system,(930) significantly increased $\mathrm{SO}_{2} \mathrm{H}-/ \mathrm{SO}_{3} \mathrm{H}$-modified peptides from proteins involved in oxidative stress response and metabolic processes.

Detection of highly charged species using mass spectrometry has its own set of complications. The high negative charge of the sulfonylated peptide can inhibit ionization by MALDI. Moreover, the sulfonic acid group can experience a neutral loss of $80 \mathrm{Da}$ by collisio-induced dissociation (CID) making the determination of specific modified cysteine residue challenging.(931) A recent alternative approach has used aryl nitroso compounds to trap sulfinic acid modification (Figure 18C).(932) A sulfinic group can form a sulfonylhydroxylamine adduct upon nucleophilic attack with an aryl nitroso compound. An electrophilic center such as an ester at the ortho-position of the aryl nitroso compound can then trap the oxyanion by intramolecular reaction thus generating a stable cyclic product 
(Figure 18C). The aryl nitroso compounds were shown to be stable in aqueous conditions $\left(t_{1 / 2}>8 \mathrm{~h}\right)$ and react with sulfinic acid groups at a first-order reaction rate of $0.31 \mathrm{~s}^{-1}$. Importantly, no cross reactivity was observed with other nucleophilic functional groups such as lysine, serine, threonine, or other oxidized thiol species such as sulfenic acid, sulfonic acid, or sulfonamide in vitro.(932) Cysteine thiols, however, do react with the probe resulting in the formation of cystine and reduced nitroso products (Figure 18C). This problem can perhaps be mitigated by first capping reduced thiols with alkylating agents such as NEM.(933) Recently, a biotinylated aryl-nitroso probe was used to detect protein sulfinylation in soluble proteome of $\mathrm{H}_{2} \mathrm{O}_{2}$-stimulated $\mathrm{HeLa}$ cells after capping all free cysteines with NEM.(933)

5.2.3 Genetically encoded probe to detect methionine oxidation-As we have alluded to above (section 3.11.1), methionine sulfoxide is a biologically relevant oxidation product.(934) Recently, the Gladyshev lab disclosed a ratiometric cpYFP-based method to measure stereospecifically the $(R)$ and $(S)$ forms of methionine sulfoxide.(934) The key insight in this method development was to fuse a stereospecific methionine sulfoxide reductase from yeast AND thioredoxin to the YFP, which become linked by a sulfide when they encounter methionine sulfoxides.

5.2.4 Identifying protein carbonylation by carbonyl capture-The reaction of hydrazine/hydrazides to aldehydes/ketones is a widely used chemical strategy to capture targets of LDE modification (Figure 19A).(935-937) Carbonyl groups on LDE-modified protein react with hydrazine to form a hydrazone adduct that requires reduction with sodium borohydride prior to downstream analysis. Hydrazine conjugated to fluorophores,(938) biotin(935) or dinitrobenzene have also been developed for determination of protein carbonylation by imaging,(938) streptavidin enrichment(939) or western blot(935) respectively. Hydrazide-biotin probe coupled with LC-MS/MS identified oxidativelyinduced protein carbonylation in brains of aged mice(940) and profile targets of various LDEs including HNE,(941) acrolein,(550) MDA and ONE (942) (Figure 19A). Global analysis of LDE-sensitive proteins upon treatment of human RKO cells with HNE, derivatization of the modified proteins using biotin hydrazide in lysate, and subsequent enrichment coupled with MS identified $>1500$ HNE-modified targets of which 400 targets showed increase in HNE adduction upon increasing HNE concentration.(941) In line with the results from the isoTOP-ABPP method,(881) protein interaction network analysis has indicated several functional cellular networks as hotspots of HNE modification including those involved in protein folding, degradation, and protein translation. $(880,881,941)$ Surprisingly, only $25 \%$ of proteins identified using competitive isoTOP-ABPP were identified using carbonyl capture method. A possible reason could be the use of MDAMB-231 in the former study and RKO cells in the latter. Nonetheless, the small overlap in the HNE-sensitive proteins identified using two different approaches exemplifies the need to exercise caution when choosing the right profiling method for an experiment.

Another proteomics method based on carbonyl capture was recently described which combines an ABPP approach with either an aminooxy probe (943) or a aniline-derived probe (944) to capture carbonylated proteins modified by LDEs. This method addresses a 
limitation of traditional ABPP by offering a direct readout of modified proteins (by LC-MS/ MS), as it does not rely on signal loss (of iodoacetamide labeling, for example) for scoring hits. Still, these latest studies further underscore the need to carefully choose a profiling probe: under identical HNE5 treatment conditions, the aniline probe captured $>1000$ carbonylated cysteines, while the aminooxy probe only captured about 300.(944)

A general limitation of carbonyl capture-based methods is that the detection of protein modification is not specific to any particular LDE. Additionally, the reactivity of the probe should also be taken into consideration. For example, dinitrophenylhydrazine (DNPH) can also react with sulfenic acid thus complicating the interpretation of results.(945) Finally, the carbonyl of the LDE may not be present post-target adduction if it is reduced or otherwise rendered non-reactive to nucleophilic carbonyl capture probes (see Section 3.9.3 and Figure 9).

\subsubsection{Profiling electrophile-responsive proteins using specific LDE-analogs-} LDE-specific analogs mitigate some of the problems of DNPH-based carbonyl capture methods (Figure 19B). They allow for profiling direct interaction of a specific LDE to proteins in the proteome. Of course, modification of the lipid can drastically change the lipid's properties. However, provided the probe is modified in a position likely to not perturb associations/reactivity too significantly and a good secondary downstream assay is available, this method can be useful. BODIPY conjugated with the electrophilic lipid $15 \mathrm{~d}-\mathrm{PGJ} \mathrm{J}_{2}$ has been used to monitor the subcellular localization of the lipid.(946-948) Additionally, biotinconjugated $15 \mathrm{~d}-\mathrm{PGJ}_{2}$ has enabled enrichment and identification of protein targets of the electrophile in cells and isolated cellular organelles.(949) $15 \mathrm{~d}-\mathrm{PGJ}_{2}$ was shown to promote calcium-induce swelling of isolated rat liver mitochondria and subsequent cytochrome-C release.(949) This induction was believed to be triggered as a result of covalent modification of various mitochondrial protein targets. Yet another group used a biotinylated probe called PLPBSO, an analog of glycerolphosphotidylcholine, an abundant phospholipid in biological membranes, supplemented to isolated human plasma to identify targets of lipid peroxidation products upon treatment with a free radical initiator. $(950,951)$ Free radical oxidation of PLPBSO results in oxidative cleavage to generate aldehydes which can modify proteins. LCMS/MS coupled analysis subsequent to enrichment of protein targets by biotin affinity pulldown revealed apolipoproteinA1 (ApoA1), the principal component of high density lipoprotein, as the most highly lipid-derived aldehyde modified protein.(951) Similarly, biotinylated AA has been used to detect targets of various LDEs generated upon cotreatment of cells with oxidants such as hemin.(948) AA generates a number of LDEs upon non-enzymatic lipid peroxidation including HNE, isoketals and isoprostanes all of which are highly reactive and can modify proteins (see section 2.2.1.2). Before using such derivatives, one must ensure that introduction of the tag does not significantly alter its physicochemical and biological properties. It is essential to have appropriate controls to delineate any artifacts associated with the tagged probes. For instance, recently a probe mimicking reactive enals was described. The probe consists of an acrylamide moiety directly conjugated to BODIPY. (952) Importantly, in the absence of conjugate addition to the enone moiety the BODIPY is dark, but once conjugation between the dye and enone systems is broken by Michael adduction, BODIPY fluorescence increases ( 350 fold). It is proposed that these sorts of 
reagents can report on endogenous reactive thiols in cells. However, to date no systematic analysis of the reactivity spectrum of these sorts of reagents has been described, and it remains unknown how even transient interactions between dyes and proteins can lead to "ligandable cysteine" type interactions. Thus, these sorts of probes must be viewed with caution.

As we stated above, biorthogonal tags such as alkyne/azido and cyclooctyne are suitable alternatives to bulky fluorescent and biotin tags (Figure 19B). The minor modification minimizes artifacts that can be observed due to even remote functionalization with bulky tags. Proteins modified with alkyne and cyclooctyne functionalized LDEs can be analyzed using in-gel fluorescence or enriched for LC-MS by conjugating with fluorophore or biotin tags using click chemistry and Staudinger ligation, respectively. Alkyne-functionalized LDEs such as HNE and ONE are now routinely used for MS profiling experiments. (537,953-955) One of the earliest examples is the use of HNE-alkyne/azide to profile HNEsensitive targets in RKO cells.(953) Cells treated with the appropriate probe were lysed and conjugated with biotin-azide/alkyne using Click chemistry. Subsequent enrichment using streptavidin beads and proteomic analysis identified several heat shock proteins and proteins involved in stress response pathways to be targets of alkyne/Azido-HNE in RKO cell lysates. A recent modification of the method used a photo-releasable streptavidin linker to conjugate to HNE alkyne-modified human plasma proteins in vitro, thus minimizing elution of nonspecific proteins bound to streptavidin beads.(955) Recently, a similar approach was used to profile protein targets of HNE and ONE added to RKO and THP-1 cells.(954) >1000 proteins were identified in each cell type to be sensitive to the electrophiles HNE and ONE. Comparison of the protein targets revealed a list of 447 proteins that are susceptible to modification with both electrophiles in both cell types. Not surprisingly, these targets comprised proteins important in various biological processes including protein translation, DNA replication, protein folding and metabolism.(954) Additionally, treatment with a range of electrophile concentrations and subsequent analyses showed that electrophile protein targets display distinct reactivity profiles in line with results obtained using competitive isoTOP-ABPP profiling methods.(884) However, only $~ 90$ of the 800 targets identified using isoTOP-ABPP were also identified in THP1 cells to be modified with HNE or ONE alkyne at any concentration. The number of overlapping targets was even lower in RKO cells (64 targets) demonstrating that the individual method chosen for MS profiling can have significant influence on the protein targets identified. A recent advancement of the method to capture protein targets of electrophiles using LDE-analogs incorporated isotopically-labeled and photocleavable azido-biotin reagent to capture and quantitatively assess protein targets of HNE alkyne in cells.(537)

\subsubsection{Targetable methods for LDE-sensitive protein screening and functional} validation-A major drawback of currently available methods to profile LDE-sensitive proteins in cells is that the methods require bolus treatment with an excess of the LDE under study from outside the cells/animals. These conditions most likely alter the redox state of the cell/organism, and trigger apoptosis. Nuances of temporal and compartmentalized signaling dynamics are also lost under bulk LDE exposure. It is thus challenging to recapitulate true spatial/temporal sensing under controlled settings using these methods. Another important 
consideration is that bolus dosing-dependent methods yield a high background of proteins that are likely not important sensors in a cellular context. Thus, distinguishing the true 'privileged sensors' from the noise generated by other contaminating proteins in the backdrop of general redox perturbation caused by excess electrophiles becomes a challenging task. Profiling experiments done by titrating a range of LDE concentrations may alleviate some of the problems associated with bolus dosing methods.(954) However, additional factors such as cellular abundance of the protein, cell permeability of the LDE, reaction of the LDE with the plasma membrane and membrane-bound proteins, cellular metabolism/excretion, etc. complicate the analysis of results obtained.

5.2.6.1 G-REX profiling of privileged LDE sensors: Recognizing these limitations, a high throughput (HT)-profiling method, termed G-REX, was recently disclosed that uses an alkyl chloride-functionalized photocaged RES precursor that binds selectively to HaloTag, a protein engineered from a bacterial dehalogenase enzyme. HaloTag shows excellent orthogonality and specificity and conjugates with the chloroalkane ligand with a secondorder rate constant of $3 \times 10^{6} \mathrm{M}^{-1} \mathrm{~s}^{-1}$,(956) resulting in 1:1 Halo-POI:photocaged LDE complex.(957) Using this strategy, a maximum of $5 \mathrm{eM}$ of HNE-alkyne was released within the cell over 5 min.(720) This concentration is tunable by controlling either the expression level of HaloTag and/or the extent of light illumination. Thus, under electrophile-limited conditions, the nascent HNE liberated at a concentration substoichiometric to that of HaloTag is competitively captured by the privileged sensors within the vicinity of Halo. As such, issues associated with metabolism/permeability are sidestepped while gaining absolute control on locale, time, dose, and chemotype of LDE released in cells. Following cell lysis, HNEylated proteins were identified using streptavidin click/biotin affinity enrichment and LC-MS/MS. Initial work involving ubiquitous HaloTag expression in HEK293T cells, namely global HNE release in situ, identified two novel HNE-sensitive proteins, Ube2V2 and Ube2V1. These proteins were responsive to HNE both in vitro and in cells and zebrafish.(720) Time will only tell how much of an advantage G-REX offers over conventional methods, and how much the presence of HaloTag and photouncaging biases targets. What is relatively clear is that G-REX and ABPP do not show huge overlap in their profiled targets, indicating the complementary nature of the two technologies.

5.2.6.2 T-REX delivery of LDEs: While existing profiling methods including G-REX altogether offer multiple unique ways to identify sensors, a method to directly link precision modifications of these sensors to functional response in living systems was non-existent until recently. To bridge this gap, 'Targetable reactive electrophiles and oxidants (T-REX)' was invented as a means to directly monitor single-POI-specific non-enzymatic modification events and downstream signaling ramifications in cells, fish and worms (Figure 20).

$(251,252,259,390,512,957-960)$ T-REX uses a proximity-driven approach (961) to release a maximally-stoichiometric amount of LDE within the microenvironment of a protein of interest (POI). Like G-REX, a genetically-encoded HaloTag is employed but it is fused to a protein-of-interest (POI).

As an initial proof of concept, two known redox-sensitive proteins, Keap1 and PTEN, were selectively modified with HNE in mammalian cells with minimal labeling of other proteins 
in the proteome.(957) Importantly, the labeling efficiency on each redox-sensor protein can be calculated using Click chemistry to conjugate a fluorescent azide molecule to the alkyne handle within the photocaged HNE conjugated to Halo and that on the HNEylated POI. The targeting efficiency on Keap1 for instance was calculated to be $~ 30-55 \%$.

$(512,609,957,959,962)$ As delivery efficiency is formally the partitioning between two unimolecular rates [pseudo-intramolecular LDE conjugation in the solvent cage (dependent on the nucleophilicity of the POI) and diffusion of LDE away from the POI (reflecting the binding capacity of the POI for the LDE)] the lower labeling efficiency typically reflects the lower electrophile sensitivity of the POI.

T-REX has also been used to unequivocally demonstrate the significance of a target-specific modification at low stoichiometry in regulating redox signaling pathways. $(259,512,958)$

Selective HNE-modification of Keap1, an adaptor protein that aids in the ubiquitination and the subsequent degradation of the transcription factor Nrf2, increased the global pool of Nrf2 in HEK293T cells.(259) Increase of Nrf2 levels led to a concomitant increase in its transcriptional activity measured using a reporter assay and using qRT-PCR to quantify the increase in transcript levels of Nrf2-driven genes. Performing T-REX in a 'non-fused' system, where the HaloTag and Keap1 were expressed as two separate proteins, did not result in any labeling of Keap1 and no resulting activation of the Nrf2 transcriptional activity. The 'non-fused' system clearly demonstrated the importance of selective modification of Keap1 using proximity enhancement (961) in Keap1 modification under TREX conditions and subsequent Nrf2-driven AR in mammalian cells. MS analysis indicated C513 and C518 as the residues modified on Halo-Keap1 in cells. Interestingly, performing T-REX in vitro modified a different set of cysteines, C226 and C368, showing that cellular environment can have drastic impact on LDE-sensitivity of different cysteine residues. Mutation of the redox-sensing cysteine residues to serine did not ablate the LDE-sensing capability of Keap1 nor upregulation in Nrf2 transcriptional activity. These data suggested Keap1 is a promiscuous LDE-sensor.(259) Indeed, earlier reports have characterized each of the 27 cysteine residues on Keap1 to be modified in vitro by at least one LDE. $(607,963)$

Beyond showing that redox signaling may regulate multiple POIs/pathways including Keap1/Nrf2/AR,(259,389,609,957) PTEN,(957) HSPB7,(964) and so on, T-REX has been expanded to include a repertoire of caged LDEs including endogenous and nonendogenously-derived electrophiles as well as simple structural mimics of $15 \mathrm{~d}-\mathrm{PGJ}_{2}$ and the electrophilic drug bardoxolone methyl (CDDO-Me).(259) Many of these compounds modified Keap1 to similar extents with very similar downstream targeting responses in mammalian cells. In vitro kinetic studies demonstrated a two-step model of electrophileadduct formation to the target: (1) formation of an initial signal-target complex, and (2) subsequent covalent conjugation of the electrophile to the POI. The labeling efficiency of POI by a specific LDE is a function of the rate of covalent adduct formation offset by the rate of diffusion of the LDE away from the initial signal-target encounter complex.

Because T-REX enables the generation of a stoichiometric amount of a given LDE in close proximity to the POI, it is an ideal tool to quantitatively assess electrophile sensitivities of putative POIs and link individual specific modifications directly to function. HaloTag human ORFeome is also commercially available facilitating such discovery efforts (Kazusa 
collection, Promega). Akt3, an isoform of the oncogenic serine-threonine kinase Akt, was recently identified as a novel sensor of HNE from a screen of a library of functionallyvalidated Halo- fused kinases.(512) Neither of the other two Akt isoforms showed comparable HNE-labeling efficiencies to Akt3. HNE modification of C119, a residue in the highly divergent flexible linker region of the enzyme (Figure 10), downregulated the kinase activity of the enzyme in mammalian cells and in zebrafish.

Importantly, the overexpression levels of Halo-Akt3 in mammalian cells was around 20- fold greater compared to basal levels whereas in zebrafish the expression levels were comparable to endogenous Akt levels. Nonetheless, similar HNEylation efficiencies and extent of downstream signal modulation were observed in both cases. These results underscore that $\mathrm{T}$ REX-assisted POI modification is entirely proximity directed quasi-intramolecular process, independent of POI expression levels. Furthermore, selective modification of only a fraction of the total POI was sufficient to trigger downstream signaling responses, suggesting a dominant-negative regulatory role of Akt3. Downregulation of the kinase activity by Akt3 HNEylation led to a consequent increase in the transcriptional activities of FOXO and p53, bona fide targets of the Akt signaling axis. Additionally, replicating T-REX on Halo-Akt2 did not yield these results. More importantly, Akt3(C119S)—sensing defective but otherwise functional kinase-was hypomorphic for all downstream phenotypes, ruling out the possibilities of off-target effects.(512)

An important advantage of T-REX over other profiling methods is that the discovery efforts can be streamlined with a unique means to validate the identified sensor robustly. $(512,959)$ Despite the powerful capabilities of chemoproteomic platforms to identify redox sensors and pin-point sites of modification on these targets, these methods do not allow modification selectively of the identified targets for functional validation. Downstream validation typically involves replicating bolus dosing treatment of knockdown cells or cells expressing LDE-insensitive mutants and subsequently measuring phenotypic response. However, such methods overlook the contribution from a number of other redox-sensitive proteins/ pathways, which are also modified using bolus dosing, to the measured response. T-REX provides a means to engender temporal and spatial control in triggering individual redox events. Additionally, T-REX is amongst only a handful of methods(958) transposable to study redox signaling in a spatiotemporally-controlled manner in various model organisms including E. coli,(959) mammalian cells,(259,957,959) C. elegans,(512) and zebrafish.(512) Together with G-REX, the dual REX technologies enable both systems-level profiling of low-occupancy privileged sensors under biologically-relevant settings as well as mechanistic interrogations into their signaling consequences at high biological resolution and target precision.

5.2.6.3 Limitations of REX technologies: On the surface, T-REX appears to be a bona fide way of identifying privileged sensor cysteines.(390,512,959) Proteins that can undergo successful modification via T-REX are relatively few; those that do sense LDEs under TREX typically undergo change of function that have phenotypically-dominant outputs. The main issue of T-REX is low throughput. This significant issue was solved by the introduction and validation of G-REX. Coupling of G-REX and T-REX makes a relatively compelling 
combination, although T-REX can in principle be used to validate any potential sensor of LDE derived from any screen.

The physicochemical basis behind T-REX/G-REX appears to be sound, as several builtin controls have shown [namely, the split construct, $(259,609)$ hypomorphic mutants, $(512)$ nolight vs. no-photocaged probe,(389) and varied transgene-expression levels in various models (512)]. However, T-REX/G-REX delivery is limited to photochemical uncaging. So far, this has proven to be a minor inconvenience as T-REX/G-REX has worked well with transparent model organisms. (Thus far, G-REX has not been tested in anything other than cell culture, although it was shown to be noninvasive in that manifold).(512) Obviously, the requirement for photouncaging will be much more of an issue for application of REXtechnologies to mice and similar organisms. Furthermore, while little evidence has arisen to show signs of UV-damage during T-REX/G-REX, $(252,259,389,512,609,720,957,960,962,964)$ it is likely that some cell types/mutants will be adversely affected by such conditions. Two-photon uncaging could be one means to avoid these issues.

For T-REX to be carried out to a specific POI, the POI must be fused to Halo. There has been extensive characterization of Halo-fused proteins, including Promega Kasuza library, $(956,965,966)$. In most instances, such chimeric proteins have proven to be functional. However, this does not mean that Halo is inert/does not exert any impact on POIs fused to it. Promisingly, where applicable, $\mathrm{C}$ and $\mathrm{N}$ terminal Halo fusions give similar delivery efficiency, allowing some flexibility.(389) Since T-REX uses ectopic expression of a Halotagged protein, there are several other potential issues: (1) the endogenous protein that cannot accept the HNE signal may suppress downstream signaling; this has proven to be a minor issue as T-REX is designed to assess phenotypically dominant (i.e., low stoichiometry) downstream signaling, events and in cell culture, at least, the target POI is overexpressed (rendering the contribution from the endogenous copy minimal; and nonetheless be easily validated using built-in controls discussed above); (2) the target POI is typically overexpressed. Requirements for overexpression, of course are their own limitation. However, T-REX works in zebrafish and $C$. elegans where Halo-POI-transgene expression is typically close to endogenous levels. Expected downstream signaling phenotypes were still observed.

Finally, it is possible that T-REX could "force" HNEylation of target POIs. In recent papers, it has been shown [in the case of HSPB7 (964) and Ube2V2 (720)] that proteins that are identified as HNE-sensitive show much faster association kinetics with HNE in vitro than known HNE-responsive proteins and proteins with known nucleophilic thiols. $(720,964)$ These data are consistent with T-REX identifying proteins that react rapidly with HNE, although more detailed assessment of reaction kinetics can help bolster this claim.

\section{$5.3 \mathrm{Immunological}$ approaches to identify RES/ROS modifications of target proteins}

Immunological methods have the potential of enhancing the selectivity and sensitivity for the detection of targets of redox signals. However, the small size of redox modification as well the large number of targets that redox signals modify has made the use of immunological methods for their detection challenging. Recent advances have allowed 
trapping of redox modifications with various chemical probes which can then be probed by antibodies as a secondary detection strategy. For example, DNPH has been used to capture carbonyl modification on proteins, which can be detected using anti-DNP antibodies in a method broadly known as OxyBlot ${ }^{\mathrm{TM}}$.(967-970) Moreover, antibodies can be used for enrichment prior to LC-MS identification of protein targets.(971) Recently this method was coupled with 2D SDS-PAGE analysis to identify proteins that are carbonylated upon premature senescence in WI-38 fibroblasts.(972)

\subsubsection{Immunological methods to detect sulfenic/sulfinic/sulfonic acid}

modifications-Sulfenic acid can be trapped using dimedone based probes. An antibody to dimedone-conjugated sulfenic acid modification has recently been developed and used to profile differential thiol oxidation in various tumor cells.(973) The antibody was also used to show that EGF signaling results in sulfenylation of catalytic cysteine of EGFR.(974) This modification is NOX2-dependent and results in the activation of the kinase. A similar antibody against $\mathrm{SOH}$-derivatized with dimedone was developed and used to study whether oxidation of GAPDH and its subsequent inactivation can cause secondary protein sulfenylation in isolated rat myocytes.(975) Antibodies to specific sulfenylated proteins such as PTPs and Prx have also been generated and/or are available commercially.(976,977) Additionally, antibodies to sulfinic or sulfonic acid modifications on specific peptides have also been developed.(696,978)

5.3.2 Antibodies to lipid aldehyde-modified proteins-Antibodies to various lipid peroxidation products have been generated. An anti-4-HNE conjugated to cysteine antibody has been used to detect lipid peroxidation product as markers of oxidative stress in cells and tissues.(979) The earliest use of immunology was to detect 4-HNE modified proteins in crude homogenates from rat liver hepatocytes treated with HNE or under oxidative stress by treating with $t$-BHQ.(979) The detection was abolished if the antibody was preincubated with HNE- $N$-acetyl lysine, HNE- $N$-acetyl histidine or HNE-glutathione, demonstrating the specificity of the antibody to HNE modified structures. Antibodies have been used to demonstrate HNE modification of I B kinase(257) and tubulin(980) with functional consequences in cells. Antibodies for a number of lipid peroxidation products such as HHEhistidine(981) and MDA-lysine(982,983) have also been developed. One interesting approach has been to develop antibodies to stable cyclic adducts of lysine. For instance, acrolein adducts to lysine form $\mathrm{N}$-(3-formyl-3,4-dehydropiperidino) lysine, likely through sequential Michael adduction followed by aldol condensation. Antibodies to this product have detected acrolein adducts in cell extracts. $(984,985)$ The 2-pentylpyrrole function is formed upon reaction of HNE with lysine. $(986,987)$ This antibody was able to detect HNElabeled human serum albumen. Similar pyrroles derived from reaction of lysine with LDEs were shown to be elevated in plasma of patients suffering from macular degeneration using a specific antibody.(988) Additionally, antibodies to $15-\mathrm{d}-\mathrm{PGJ}_{2}$ have been used to detect this prostaglandin in cells using IHC and show proteins modified by $15-\mathrm{d}-\mathrm{PGJ}_{2}$ using ELISA. $(266,989)$

Antibodies are useful to detect specific protein modifications, yet they often face problems of cross-reactivity. For instance, anti-HNE antibodies cross-react with 4-hydroxy-2-decenal, 
4-hydroxy-2-octenals well as lysine-ONE modifications. (571,990) Additionally, antibodies may be limited to detecting only one form of modification to a particular LDE. HNE, for example, has two reactive groups. It can form a direct cysteine adduct at $\mathrm{C} 3$ and/or react with another lysine at the carbonyl moiety of the free aldehyde group resulting in a Schiff base. Additionally, HNE adducts can also lead to intramolecular cyclization forming a cyclic product (Figure 13B). An antibody specific to a particular form may not detect other forms thus introducing significant bias and limiting results. Additionally, antibodies do not provide information on the site selectivity of these redox modifications. Antibody-based enrichment coupled with digest mass spectrometry can mitigate this issue.(971)

\section{RES/ROS SIGNALING IN DISEASE AND THERAPEUTIC INTERVENTIONS}

We will lay out below a case for RES/ROS signaling being important in health and disease. Data hinting at correlations between RES/ROS signaling and (un)wellness have been around for many years. However, as we describe most of these reports are circumstantial and require significant leaps of faith to ascribe any specific phenotype to any specific (or even general) RES/ROS chemotype.(509) In general, in this field, RES are often used as a proxy for ROS. However, the situation is often complicated, and there is significant evidence indicating that RES contribute directly to disease states. In this specific context, our discussion is centered on HNE as the best-studied endogenous RES. In line with an overall focus on RES/ROSbased therapeutics, we devote this section to broad-specificity small-molecule electrophilic drugs either approved or currently/previously in clinical trials (Figure 21). Many of these pharmacophores tap into redox-modulated pathways. We first examine the broader pathological roles of these native and synthetic reactive small molecules in various disease states, with emphasis on cancer, diabetes, neurodegeneration, and multiple sclerosis. It is ultimately likely that the pleotropic nature of RES and ROS, and the fact that they occur typically in tandem mean that healthy and disease phenotypes are attributable to multiple RES/ROS acting in concert. We finally analyze, using Nrf2, FOXO, and Wnt/ $\beta$-catenin signaling as case examples, the role of crosstalk between RES/ROS-responsive proteins regulating various signal transduction pathways. Building on these latest findings, we discuss how RES/ROS-signal wiring may be altered to accommodate a specific pathological state, and how this emerging knowledge about context-specific alterations in pathway intersections may be exploitable for novel interventions. One of the key motivations of this chapter is to illustrate how redox signaling in general is a complex process that regulates numerous pathways and exerts its influence on many signaling nodes above and beyond the Nrf2/AR pathway.

\subsection{Diabetes}

Several studies have implicated high ROS levels in diabetes etiology. Type II diabetes, for instance, is exacerbated by elevated ROS levels(991) that are caused by hyperglycemia, increased non-enzymatic glycosylation, and activation of the ETC.(992) ROS elevation in turn causes several responses including, decreased insulin expression and upregulation of stress-activated protein kinase/c-Jun amino-terminal kinase (JNK).(993,994) 
Unsurprisingly, the extent of RES-adducted proteins also increases in obesity and diabetes. (995) 4-HNE levels are also elevated in the non-diabetic obese population.(996) This may be partly caused by a decrease in GST-a4(997) and concomitant decrease in HNE metabolism. (998) HNE upregulation has several effects associated with diabetes including promoting adiposeness and insulin resistance.(999) Consistent with this argument, uncontrolled lipid oxidation products are associated with uncontrolled diabetes.(1000)

One of the best studied pathways of stress relevance in disease including diabetic models is AR. CDDO-Me and some analogs, which are all potent steroid-derived AR stimulators, have shown potency in animal models of diabetes (Figure 22).(1001) CDDO-Me reached clinical trials for its ability to suppress end-stage renal disease, the most advanced stage of kidney disease where diabetes mellitus is a key risk factor. Unfortunately, little therapeutic effect was found. In addition, deleterious off-target effects(1002-1004) were also reported. Optimization of CDDO-Me, or possibly, use of alternative Nrf2-stabilizing motifs(1005) may improve efficacy while alleviating off-target effects, although more work is required in this area.(1006)

\subsection{Neurodegeneration}

Neurodegeneration is linked to elevated RES/ROS in neuronal cells. $(1007,1008)$ Elevated oxidative stress markers have been detected in brains of patients with Alzheimer's disease, (315) Parkinson's disease,(1009) in amyloid-beta plaques,(1010) in cerebrospinal fluid of patients with amyotrophic lateral sclerosis (ALS; Lou Gehrig's disease),(1011) and in Huntington's disease.(1012) However, there is significant debate as to whether ROS is a cause or a consequence of these diseases. As we will see below, recent work has tried to use modern chemical biology techniques to understand ROS signaling and develop a quantitative understanding, although there is still a long way to go in this area. On the other hand, genetics experiments have shown that Nrf2 knockout significantly increases learning and memory defects in Alzheimer's disease model mice.(1013) Subsequent work has shown that Nrf2 knockout mimics mRNA changes that occur during Alzheimer's disease.(1014) These outcomes could also be due to non-ROS/RES-related functions of Nrf2. However, it is hard to ignore the strong relationship between Nrf2-driven AR and suppression of RES/ROS elevation. Unfortunately, neurons are extremely complex and transposition of model systems to humans has proven challenging. But numerous model systems for neurodegenerative diseases exist, including yeast,(1015) mice, fruit fly, worms(1016) and organoids,(1017) so there is much scope for experimentation and developing understanding.

ROS elevation appears to be an early step in many neurodegenerative diseases (Figure 22). However, exactly how ROS is elevated is less obvious. For instance, in Alzheimer's disease, amyloid- $\beta$ plaques contribute to elevated ROS. However, in vitro, the opposite is the case, i.e., plaques have low ROS-forming potential.(1018) In addition to amyloid- $\beta$, ROS may be activated through: metal chelation,(1019) mitochondrial defects,(1020) ER stress,(168) and microglia priming.(1021) Furthermore, downstream targets of ROS are also somewhat unclear. ROS has been postulated to: activate c-Abl,(1022) decrease/inhibit autophagy, (1023) stimulate inflammation,(1024) and modulate ubiquitin pathways (including proteolysis).(1025) 
Regardless of the mechanism of generation/action, numerous models of neurodegenerative diseases have indicated that reduction in ROS levels can improve cognitive function and survival. Unfortunately, the effects of antioxidants supplements/drugs are often less impactful on humans than in model systems. In Huntington's disease, for instance, numerous model systems are improved by treatment with antioxidants, $(1026,1027)$ including a-lipoic acid,(1028) cystamine,(1029) coenzyme Q10,(1030) and plant-derived antioxidants.(1031) However, this strategy is not particularly effective in human patients, (1032-1034) although some unexpected long-term benefits may occur. Furthermore, new data using a $C$. elegans model suggest that oxidative stress is not a significant causative factor in Huntington's disease.(1035) That being said, Edaravone (Figure S3) was recently approved to treat ALS. Edaravone is believed to have antioxidant properties,(1036) although these have not yet been directly tied to its mechanism.(1037)

Nrf2 activation may protect from neurodegeneration (Figure 22). In a Drosophila melanogaster model of Parkinson's disease, for instance, elevation of Nrf2 (cncC, in $D$. melanogaster) signaling suppressed neurodegenerative changes. In this instance, $\mathrm{cncC}$ was upregulated either through treatment with CDDO-Me or through dopaminergic neuronspecific expression of cncC. Importantly, using a roGFP2-based probe (see section 5.1.4) the authors correlated severity of behavioral disorder with ROS levels.(1038) Similar protective effects of synthetic triterpinoids such as CDDO-ethyl amide and CDDO-trifluorethyl amide were found in mice.(1039) Some authors have proposed Nrf2 activation as a global means to treat all neurological damage.(1040)

HNE levels are increased in brain and body fluids of patients with various neurodegenerative disorders. High HNE levels can increase brain damage.(1041) The mechanisms of HNE causing brain damage are manifold: HNE is implicated in increasing aggregation of asynuclein and export of aggregated a-synuclein to healthy neurons; lysosome dysfunction; (1042) and GSH depletion.(1043) Interestingly, quercetin, a polyphenolic plant-derived compound that is able to elevate GSH levels(1044) in the brain can alleviate Alzheimer's disease symptoms in mice.(1045) These studies may translate to human patients: for instance, recent investigations indicate that the well-known neurological protective properties of coffee can be traced to quercetin(1046) (Figure S3); and quercetin can reduce HNEylation of proteins in neuronal culture.(1047)

Of course, these studies all assume a single cause and effect model of disease. In many instances this is more or less true and has led to many successful therapies: imatinib, an inhibitor of Bcr-Abl, an oncogenic fusion kinase responsible for chronic myeloid leukemia is a classic example.(1048) However, it is likely that many factors are responsible for neurodegeneration etiology, and hence, ROS suppression and/or electrophile detoxification are not sufficient to provide a large therapeutic effect.(1049) It is also possible that many of the model systems where these putative therapies are effective are not as complex as the actual disease in humans. This short-coming is starting to be addressed by development of multi-targeting molecules, such as BN82451 (Figure S3).(1050) This phenolic compound is anti-inflammatory, antioxidant and blocks sodium channels. Of course, combination therapies are another strategy to combat multifaceted diseases, and such regimens are being investigated for numerous brain diseases.(1051,1052) 


\subsection{Multiple sclerosis}

ROS detoxification appears to be impaired in multiple sclerosis (MS) patients.(1053) ROSinduced damage and protein RES modification(1054) occur in the brains of MS patients. (1055) Modulation of ROS signaling is postulated to be an effective therapy against MS. (1056) However, as we commented above, antioxidants have not been particularly successful in treating MS, although they have fared better in mouse models.(1057)

One of the latest promising developments in MS treatment is the FDA approval (in 2015) and subsequent accelerating sales of the electrophilic Nrf2 agonist Tecfidera (dimethyl fumarate) (Figure 22).(1058) The mechanism of action of this drug continues to be debated. However, its ability to stimulate Nrf2 is likely not the only reason behind its clinical activity. $(1059,1060)$ Recent investigations into the effect of Tecfidera on mice showed that Nrf2 was not required for the pharmacological effects of Tecfidera. Independent studies have further confirmed that Tecfidera's T-cell stimulation activity is independent of Nrf2.(1060,1061) There is one caveat to this analysis: Nrf1 can at least partially compensate for Nrf2 function in these cells,(1062) meaning AR regulation may not be fully compromised in these models. ABPP indicated several targets of Tecfidera, one of which is protein kinase $\mathrm{C} \theta$. It is noteworthy that protein kinase $\mathrm{C}$ interacts with HIF-1a, a protein that is destabilized by Tecfidera.(1063) It is not known how loss of HIF-1a affects nucleophilicity/activity of protein kinase C. Another recent study showed succination of GAPDH by Tecfidera to be important in mediating its therapeutic effect in mice model of MS.(1060) Clearly, the promiscuity of reactive electrophilic drugs, like Tecfidera, renders it difficult to understand specific targets and delineate a precise molecular mechanism.

\subsection{Context-specific shifts in RES/ROS signaling crosstalk}

RES/ROS regulation permeates every physiological process/pathway known (metabolism, protein quality control, epigenetics, and transcriptional regulation, and so on). Indeed, innumerable proteins of diverse function and cellular roles are now implicated as RES/ROS sensors. Recent evidence on balance indicates that RES/ROS sensing/signaling is best regarded as a protein moon-lighting function.(251,390,509) These activities are some of the most interesting carried out by enzymes. Unfortunately, they are particularly difficult to predict and understand. The diverse repertoire of individual redox-influenced pathways has been documented and we refer the readers to these excellent reviews.

$(5,31,115,505,866,1064-1067)$

However, cell signaling networks operate non-autonomously. These connective mechanisms are also often altered in specific disease states. Given that (i) cysteine mutations occur most frequently among coding residue mutations within disease database, $(390,1068)$ and (ii) there is a growing body of data connecting specific cysteines to specific disease-relevant states, altered RES/ROS sensing/signaling likely plays an important role in numerous disease etiologies. In this sub-section, we explore functional redox crosstalk, with the focus on two key evolutionarily-conserved pathways/intersections: Nrf2/AR crosstalk with (i) Wnt signaling; and (ii) EGF signaling. These examples make a strong case that gleaning precise understanding of RES/ROS signaling mechanisms can help us to gain new knowledge of basal signaling patterns, pathway intersections, and disease etiology. The EGF system 
additionally represents the first establishment of physiological $\mathrm{H}_{2} \mathrm{O}_{2}$ signaling and both RES/ROS and several approved drugs uniquely tap into specific kinase sensors/isoforms that regulate this signaling subsystem.

Having described the dichotomous relationship between on- and off-target effects in natural and non-natural reactive species in disease, these specific examples provide a more focused lens into disease-relevant shifts in pathway interconnections. We stress that these insights arose from studying protein specific RES signaling. $(252,512,720)$ We conclude that such surgical interrogations hold promise toward improved targeted intervention of druggable pathways as well as novel covalent ligand discovery targeting the hitherto undruggable proteome.

\subsection{Cancer}

Deregulated redox homeostasis has been described as a hallmark of cancer cells.(1069)

These changes in RES/ROS levels are typically driven by genetic modifications that promote stress. However, cancer is a complex disease encompassing multiple stages of "development"(1070) and it was for some time unclear whether ROS elevation was an end product of oncogenesis or whether it is active in the transformation program. We therefore discuss the role of RES/ROS in the various stages of oncogenesis sequentially.(1071)

\subsubsection{Moderate ROS elevation promotes oncogenic transformation through} multiple pathways-As we have noted, moderate levels of ROS elevation are commonly associated with promotion of cell growth. Many proliferative stem cells show elevated ROS levels(1072) and various oxygenase enzymes are critical for correct development.(1073) Indeed, ROS have complex effects on mammalian development, $(1074,1075)$ with both upregulated and down-regulated ROS levels occurring in a coordinated fashion. Intriguingly, many of the processes that occur during embryonic development are similar to those that occur during cancer development, $(1076,1077)$ further implying that ROS levels will be important in cancer development and progress.

ROS function in early stages of oncogenesis to help create an environment conducive for growth and transformation, i.e., overall leads to a mutator phenotype.(1078) ROS elevation has been observed upon overexpression of single oncogenes in both cell culture(1079) and model organisms.(1080) ROS elevation can speed transformation by promoting genome instability(1081) that can help to drive mutations essential for cancer progression(1082) in what is typically, but not always, a gradual process.(1083,1084) Furthermore, ROS may serve to modulate DNA damage repair pathways, further promoting mutation. In addition, ROS also directly and indirectly regulate tumor suppressor genes, such as p53. It has recently been shown that impairment of the ETC or decrease in mitochondrial DNA can promote ROS generation that leads to a genetic inactivation of p53.(1085) As a result, cells show much higher proliferative ability and greater susceptibility to neoplastic transformation. Unsurprisingly, several tumor suppressors have an antioxidant function. $(1086,1087)$ Furthermore, some labs have reported that oncogenic transformation of mesenchymal stem cells involves a decrease in Nrf2 levels and decrease in antioxidant 
genes.(1088) Although such changes are likely to affect manifold pathways, ROS levels would likely increase.

However, ROS levels must not be too high.(1089) Hyper-elevated ROS levels may contribute to oncogene-induced senescence. Certain cancer stem cells, for instance, require Nrf2 to retain their self-renewal properties. $(1090,1091)$ Unsurprisingly, many oncogenes stimulate Nrf2 upregulation.(1091-1093) Furthermore, careful experiments looking at the effect of antioxidants on oncogenic RAS transformation showed that acetyl cysteine suppressed RAS oncogene-induced senescence, partly through slowing down ROS-promoted growth elevation, allowing continued sustained growth.(1080) Thus, ROS levels likely need fine tuning and must to able to accommodate competition for space, resources, etc. that occur during tumor growth.(1094)

6.5.2 RES may serve a natural tumor-suppressor function-Early studies on tumor formation potential of HNE and a more reactive analog 2,3-epoxy-4-hydroxynonanal showed that both species have little detectable carcinogenic potential in mice.(1095) However, these electrophiles have been linked to DNA damage behavior including sister chromatid exchange, and mutagenesis.(1095,1096) There is also evidence that HNE can selectively cause mutation of the tumor-suppressor p53 through directly modifying genomic DNA.(1097) RES levels are strongly correlated with ROS levels, and hence RES should also increase in cancer. Indeed, some studies have found elevation of RES in blood of cancer patients, indicating that redox signaling can be impacted in whole organisms by cancer. (1098) However, this observation does not seem to be recapitulated on a cancer cell-specific level: few studies have been performed on how RES affect oncogenesis, but, there appears to be a general, although not universal,(1099,1100) decrease in lipid peroxidation products during cancer development.(1101-1104) Although how RES affects cancer cells in model organisms and humans is still not fully clear, it is quite well established that HNE exerts a negative effect on many, but not all,(1105) mature cancer cell growth [including K562(1106), various colon cancer cells(1107) and HL-60;(1108) with $\mathrm{IC}_{50}$ 's in the mid- to low-micromolar range)] while being less impactful on non-transformed cells, implying that HNE would overall countermand oncogenesis. Indeed, far from being toxic to nontransformed cells, HNE stimulates growth of vascular and aortic smooth muscle cells in the mid to low micromolar range. $(1109,1110)$

Indeed, HNE reduces cachexia (weight loss/tissue wastage) in a lung cancer model,(1111) principally by exerting a selective growth inhibition effect on cancerous cells. Interestingly, it has been reported that overexpression of the HNE-detoxifying enzyme, GST- $\alpha$, is sufficient to transform at least some cell types (e.g., human lens epithelial cells). Importantly, the transformation potential of GST is correlated with its ability to metabolize HNE and can be affected by heterologous expression of non-human proteins. Transformed GST-a-overexpressing cells showed reduced HNE levels, which led the authors to postulate that HNE reduction is sufficient to promote transformation.(1112) Other authors have postulated that sulforaphane, a well-known cancer preventing (prophylactic) agent (Figure 21) may function partially through upregulation of cellular HNE(1113) (although it is indisputable that other mechanisms also contribute to lowering cancer incidences). Glutathione depletion, which would raise HNE levels, also suppresses growth of cancer 
cells. Broadly speaking, most cancer cells upregulate GST expression,(1114) which may also indicate a drive to suppress RES signaling. However, the need to protect from elevated ROS/RNS, etc. cannot be ruled out and the potential roles of ROS/RNS are also difficult to parse from RES-specific effects in these experimental models. Thus, ultimately, little or no direct evidence for the specific involvement of HNE in these processes has been provided. Given that effects of endogenous RES such as HNE show a complex dose response(1115) and enzymes responsible for HNE maintenance also regulate ROS metabolism, it may indeed ultimately transpire to be very difficult to obtain direct evidence for the pathological effects of HNE. Furthermore, some cancer cells, such as MCF7, appear to express very little of the canonical GST isoforms $(\alpha, \mu$ and $\pi)$.

It has been known for 20 or more years that several physiological changes that may be expected to suppress lipid signaling occur during oncogenic progression.(1116) Briefly, cell membranes of cancer cells switch to a more saturated [less fluid and less readily oxidizable(1117)] state.(1118) Lipid pathways are some of the most altered metabolic systems in for instance pancreatic tumors.(1119) That being said, it is widely appreciated that membranes are important for numerous cellular processes, including signaling and permeation of small molecules. It thus remains very difficult to tie lipid changes specifically to suppression of RES signaling along the oncogenic pathway.(1120)

\subsubsection{Mature cancer cells also rely on ROS, but are susceptible to elevated}

ROS-Mature cancer cells continue to be subject to high levels of oxidative stress. A recent study, for instance, found a correlation between metastatic spread and basal ROS levels. (1121) Indeed, ROS elevation assists several steps within the advanced oncogenic program, including angiogenesis [in part through stabilizing hypoxia inducible factor (HIF), (1122) avoidance of apoptosis,(1123) and metastasis(1124,1125)]. Several studies link elevated ROS levels to increased disease progression and poor clinical outcomes.(996) However, several emerging strategies are geared to take advantage of high ROS levels in cancer to selectively push diseased cells over the edge.(1126) Piperlongumine (Figure S3), a reactive electrophilic molecule studied by the Schreiber lab, was shown to inhibit GST-p and cause increase in ROS levels and cell death selectively in cancer cells. (1079) The authors conclude from this study that GST-p is an example of non-oncogene addictive behavior: i.e., cancers rely on GST-p much more than normal cells, although (by this definition) GST is not sufficient to cause transformation. These data are in broad/general agreement with studies above on GST-a-promoted transformation, although the data indicate a more passive role in oncogenesis. Similarly, it has been postulated that phenethyl-ITC (Figure 21) can kill cancer cells through a ROS-dependent mechanism.(1127) Some pharmaceuticals modulate RES/ROS in a locale/organelle-specific manner. One such drug is doxorubicin (Figure S3) (approved to treat breast and bladder cancer amongst other). This drug elevates cellular HNE levels, and increases in mitochondrial HNE-modified proteins have been detected in cells treated with doxorubicin.(1128)

\subsubsection{RES have complex and diverse effects on mature cancer cells, but are principally anticancer-Interestingly, HNE is postulated to promote angiogenesis} through a similar mechanism to ROS (i.e., increase in HIF).(1129) In the case of HNE, 
however, angiogenesis is promoted through inhibition of Sirt3, mitochondrial sirtuin deacetylase in mammals. Consistent with this proposal, HNE could not promote angiogenesis in Sirt3-knockdown cells. An analogous compound, 4-hydroxy-7-oxo-5heptenoic acid lactone (Figure S3), also promotes angiogenesis.(1130) In spite of angiogenesis activity, RES would be predicted to be a particularly potent weapon against cancer, given that some studies implicate RES as a natural anticancer prophylactic (see also Section 6.5.2). Sulforaphane and other dietary ITCs are in clinical trials to treat several cancers. However, it seems likely that the therapeutic window of reactive lipids like HNE is too small to be used directly. To this end, inhibition of HNE-metabolizing enzymes has been proposed as a means to upregulate HNE and kill cancer cells.(1131) Several broadly-reactive carbon-based electrophiles are currently used in clinic or are in clinical trials for cancer treatment. Compounds used in the clinic include nitrogen mustards (mechlorethamine, or carmustine), disulfonate cross-linkers (Busulfan) and dacarbazine (Figure S3). Several of these electrophiles stimulate ROS production and this has been proposed to contribute to their pharmaceutical program.(1132) Clinically-trialed electrophiles include VLX1570 (Figure 21) and melflufen (Figure S3). VLX1570 and other analogs stimulate Nrf2 activity and upregulate heme oxygenase 1 (HMOX-1),(1133) although the mechanism is unknown, as is how this function contributes to efficacy. Furthermore, some authors have proposed that the potent Nrf2 agonist, Tecfidera, can be repurposed to treat cancer,(1134) and the drug has shown efficacy in xenograft tumor models.(1135)

As discussed above, several drugs in clinic now have weakly-reactive electrophilic appendages (commonly acrylamide) (Figure 21). These appendages, in contrast to the enones, sulfonates, and chloramines discussed above, are of relatively low promiscuity and (ideally) engage with target cysteine(s) only when pre-bound to target enzyme(s). The irreversible binding mode brings several pharmacological advantages relative to noncovalent equivalents. Although unlikely a trait selected during oncogenesis, it has been shown that high ROS levels can oxidize these cysteines, reducing potency of the inhibitors. We recently proposed that modification of this strategy could be used to direct HNE to a specific protein of interest to tap into endogenous lipid signaling pathways that could confer benefits over "normal" drug targeting pathways.(390) No such drugs have yet been reported.

6.5.5 Nrf2 crosstalk with Wnt signaling-As alluded to earlier in this chapter, Nrf2/AR upregulation is a critical component of numerous diseases. It is known that modulations in Nrf2 leads to significant pathway rewiring that may expose an Achilles heels that can be exploited for therapy.(893) However, Nrf2 regulation and pathway intersections are synergistically developed during disease progression, so it is very likely that a personalized approach to Nrf2/AR signaling should be taken. Here we discuss recent identification of redox-dependent crosstalk between Nrf2 and Wnt, another pathway commonly upregulated in cancer.(1136)

$\beta$-catenin (a transcription factor driving canonical Wnt signaling) and Nrf2 are both substrates of $\beta$-TrCP, an E3 ubiquitin ligase responsible for K48-linked ubiquitination, leading to proteasomal degradation of client proteins. Thus, $\beta$-catenin and Nrf 2 share a common regulator, indicating that Wnt and AR may crosstalk. Indeed, similarly to Nrf2, Wnt signaling upregulation commonly occurs in cancers. However, upregulation of Wnt 
occurs by loss of the ability of $\beta$-TrCP to bind to $\beta$-catenin. Numerous reports indicate that Wnt signaling can stimulate Nrf2 and hence upregulate AR.(1137) However, it was less clear how Nrf2 affects Wnt. Using T-REX-assisted HNEylation of Keap1, it was found that $\beta$-TrCP knockdown led to a suppression of AR.(252) This suppression was not observed following bulk HNE exposure of cells, likely because of off-target effects. Subsequent experimentation showed that the inhibition of Wnt signaling by Nrf2 was increased when $\beta$ TrCP could not bind $\beta$-catenin: in $\beta$-catenin knockdown cells, the elevation of $\mathrm{Nrf} 2$ induced by selective HNEylation of Keap1 alone is significantly offset by the fact that Wnt signaling is highly susceptible to Nrf2 inhibition, and Wnt inhibition leads to decrease in AR. Thus, there is shift in the interplay of Nrf2 and Wnt during cancer progression and mature cancer cells could be sensitive to AR upregulation, provided they upregulate Wnt through $\beta$-catenin $\mathrm{N}$-terminal deletion/mutation. Although no systematic study has been performed, it is possible that this relationship could help guide the usage of Nrf2 stimulating drugs in anticancer therapy. Several studies are in concordance with this hypothesis (Figure 23). (1138)

\subsubsection{Nrf2 crosstalk with EGF signaling along PI3K/Akt/FOXO axis-An} interesting positive feedback loop between Nrf2 and EGFR is slowly coming into focus (Figure 23). It is established that EGFR upregulation either upon endogenous stimulation or by constitutive activation leads to Nrf2 upregulation.(1093) This pathway could occur through modification of Keap1(1139) although the more likely cause is that EGFR stimulates Akt3,(1140,1141) which can in turn inhibit GSK3 $\beta$, a negative regulator of Nrf2. It was also found that Nrf2 can also positively regulate Notch,(1142) a factor that positively regulates transcription of EGFR.(1143)

As we have discussed, ROS levels are elevated in cancer yet balanced to promote cell growth, without triggering senescence. There is evidence that ROS elevated stimulation of EGFR can directly stimulate Nrf2 and ARE genes. In fact, EGFR inhibition can prevent Nrf2 activation during hyperoxia.(1144) Similarly, inhibition of PI3K, a protein downstream of EGFR, also decreases Nrf2 levels.(1145) Indeed, ROS also inactivates PTEN, a negative regulator of the PI3K pathway. Thus, it is likely that ROS elevation has a pleotropic effect on multiple pathways, probably in a reinforcing manner. It may be critical that such reinforcing pathways exist, as ROS elevation in cancers can originate from numerous sources(1146) and it is likely that the origin of ROS generation can affect the order of pathway stimulation. For instance, NOX2-mediated stimulation/inhibition of signaling pathways through ROS modification of specific kinases follows the distance from the point of $\mathrm{H}_{2} \mathrm{O}_{2}$ generation: EGFR is closest to NOX2 and is preferentially modified by ROS, whereas PTEN and PTPB1 are further away from the source, so oxidation is less likely. Although this analysis assumes that proximity to ROS rather than second-order kinetics or reaction of peroxide with a specific protein determines modification selectivity, it is certainly interesting to consider that context will control which pathway is modified.(1147) Clearly expression levels of the specific proteins involved, and detoxification mechanisms will also play a big role in determining how ROS influence cell growth and through which pathways. 


\section{OUTLOOK}

The literature abounds with preclinical and clinical studies on antioxidant/RES-related drugs/regimens.(1148) However, ultimately, few ever make it to the clinic or show efficacy in trials. Furthermore, for many years, electrophilic drugs were almost considered anathema in drug development.(1149) However, with the new-found appreciation for covalent inhibitors in clinical trials and front-line drugs, as well as the appreciation of multiple-target drugs,(1150) there has at least been a renaissance of electrophilic elements in drug design. (390,1149,1151-1154) Clearly the unresolved issue with antioxidants, and notion that cysteines have now become druggable, reaffirms the importance of identifying privileged sensor cysteines, and ligandable cysteines that are primed for drug development. We believe that study of redox signaling pathways can help illuminate and guide both discovery of electrophilic drugs and hone our efforts on the most beneficial drug targets. It is thus of paramount importance to gain a comprehensive overview of the precision cysteome dynamics and its overarching influence on cellular processes. Developing such a "redox signaling atlas" is one of the key scientific challenges of this century.

\section{Supplementary Material}

Refer to Web version on PubMed Central for supplementary material.

\section{Acknowledgments}

The following organizations are acknowledged for supporting our research projects: NSF CAREER (CHE-1351400), Beckman Young Investigator, NIH-New-Innovator (1DP2GM114850), Office of Naval Research (ONR) Young Investigator (N00014-17-1-2529), Burroughs Wellcome CRTG, and the Sloan fellowship (FG-2016-6379), and Pershing Square Sohn Cancer Research Alliance programs to Y.A., HHMI pre-doctoral fellowship to S.P.; AHA predoctoral fellowship (17PRE33670395) and Cornell Chemistry Biology Interface (CBI) training grant (NIGMS T32GM008500) to J.R.P.; zebrafish husbandry and microinjection/imaging facility (NIHR01 NS026539, PI: J. Fetcho), Cornell NMR facility (NSF MRI: CHE-1531632, PI: Y.A.), Cornell Imaging Center (NIH 1S10RR025502, PI: R.M. Williams), Cornell proteomics facility (NIH SIG grant 1S10RR025449-01) for resources and instrumentation.

\section{References}

1. Baker MJ, Frazier AE, Gulbis JM, Ryan MT. Mitochondrial Protein-Import Machinery: Correlating Structure with Function. Trends Cell Biol. 2007; 17:456-464. [PubMed: 17825565]

2. Wishart DS, Jewison T, Guo AC, Wilson M, Knox C, Liu Y, Djoumbou Y, Mandal R, Aziat F, Dong E, et al. HMDB 3.0-The Human Metabolome Database in 2013. Nucleic Acids Res. 2013; 41:D801-D807. [PubMed: 23161693]

3. Lindahl T, Barnes DE. Repair of Endogenous DNA Damage. Cold Spring Harb Symp Quant Biol. 2000; 65:127-134. [PubMed: 12760027]

4. Smith BC, Marletta MA. Mechanisms of S-Nitrosothiol Formation and Selectivity in Nitric Oxide Signaling. Curr Opin Chem Biol. 2012; 16:498-506. [PubMed: 23127359]

5. Paulsen CE, Carroll KS. Cysteine-Mediated Redox Signaling: Chemistry, Biology, and Tools for Discovery. Chem Rev. 2013; 113:4633-4679. [PubMed: 23514336]

6. Hess DT, Matsumoto A, Kim SO, Marshall HE, Stamler JS. Protein S-Nitrosylation: Purview and Parameters. Nat Rev Mol Cell Biol. 2005; 6:150. [PubMed: 15688001]

7. Filipovic MR, Zivanovic J, Alvarez B, Banerjee R. Chemical Biology of H2S Signaling Through Persulfidation. Chem Rev. 2018; 118:1253-1337. [PubMed: 29112440]

8. Mishanina TV, Libiad M, Banerjee R. Biogenesis of Reactive Sulfur Species for Signaling by Hydrogen Sulfide Oxidation Pathways. Nat Chem Biol. 2015; 11:457-464. [PubMed: 26083070] 
9. Giles GI, Nasim MJ, Ali W, Jacob C. The Reactive Sulfur Species Concept: 15 Years On. Antioxidants. 2017; 6:38.

10. Marnett LJ, Riggins JN, West JD. Endogenous Generation of Reactive Oxidants and Electrophiles and their Reactions with DNA and Protein. J Clin Invest. 2003; 111:583-593. [PubMed: 12618510]

11. Hwa Lee S, Rangiah K, Williams MV, Wehr AY, Dubois RN, Blair IA. Cyclooxygenase-2Mediated Metabolism of Arachidonic Acid to 15-Oxo-Eicosatetraenoic Acid by Rat Intestinal Epithelial Cells. Chem Res Toxicol. 2007; 20:1665-1675. [PubMed: 17910482]

12. Groeger AL, Cipollina C, Cole MP, Woodcock SR, Bonacci G, Rudolph TK, Rudolph V, Freeman BA, Schopfer FJ. Cyclooxygenase-2 Generates Anti-Inflammatory Mediators from Omega-3 Fatty Acids. Nat Chem Biol. 2010; 6:433-441. [PubMed: 20436486]

13. Clark SR, Guy CJ, Scurr MJ, Taylor PR, Kift-Morgan AP, Hammond VJ, Thomas CP, Coles B, Roberts GW, Eberl M, et al. Esterified Eicosanoids are Acutely Generated by 5-Lipoxygenase in Primary Human Neutrophils and in Human and Murine Infection. Blood. 2011; 117:2033-2043. [PubMed: 21177434]

14. Morgan Lloyd tThomas Christopher pKühn H, O'donnell Valerie b. Thrombin-Activated Human Platelets Acutely Generate Oxidized Docosahexaenoic-Acid-Containing Phospholipids via 12Lipoxygenase. Biochem J. 2010; 431:141-148. [PubMed: 20653566]

15. Jin J, Zheng Y, Brash AR. Demonstration of HNE-Related Aldehyde Formation via LipoxygenaseCatalyzed Synthesis of a Bis-Allylic Dihydroperoxide Intermediate. Chem Res Toxicol. 2013; 26:896-903. [PubMed: 23668325]

16. Michelucci A, Cordes T, Ghelfi J, Pailot A, Reiling N, Goldmann O, Binz T, Wegner A, Tallam A, Rausell A, et al. Immune-Responsive Gene 1 Protein Links Metabolism to Immunity by Catalyzing Itaconic Acid Production. Proc Natl Acad Sci U S A. 2013; 110:7820-7825. [PubMed: 23610393]

17. Bindoli A, Rigobello MP. Principles in Redox Signaling: From Chemistry to Functional Significance. Antioxid Redox Signal. 2013; 18:1557-1593. [PubMed: 23244515]

18. Krumova K, Cosa G. Singlet Oxygen: Applications in Biosciences and Nanosciences. Nonell S, Flors C, editors Vol. 1. The Royal Society of Chemistry; Cambridge, UK: 2016.

19. Tyler DD. Polarographic Assay and Intracellular Distribution of Superoxide Dismutase in Rat Liver. Biochem J. 1975; 147:493-504. [PubMed: 810138]

20. Lundgren CaKSjostrand D, Biner O, Bennett M, Rudling A, Johansson AL, Brzezinski P, Carlsson J, Von Ballmoos C, Hogbom M. Scavenging of Superoxide by a Membrane-Bound Superoxide Oxidase. Nat Chem Biol. 2018; In Press. doi: 10.1038/s41589-41018-40072-x

21. Wenzel S, Taimor G, Piper HM, Schlüter KD. Redox-Sensitve Intermediates Mediate Angiotensin II-Induced p38 Map Kinase Activation, AP-1 Binding Activity, and TGF- $\beta$ Expression in Adult Ventricular Cardiomyocytes. FASEB J. 2001; 15:2991-2993.

22. Zimmerman MC, Lazartigues E, Lang JA, Sinnayah P, Ahmad IM, Spitz DR, Davisson RL. Superoxide Mediates the Actions of Angiotensin II in the Central Nervous System. Circ Res. 2002; 91:1038-1045. [PubMed: 12456490]

23. Yang W, Hekimi S. A Mitochondrial Superoxide Signal Triggers Increased Longevity in Caenorhabditis elegans. PLoS Biol. 2010; 8:e1000556. [PubMed: 21151885]

24. Giorgio M, Trinei M, Migliaccio E, Pelicci PG. Hydrogen Peroxide: A Metabolic By-Product or a Common Mediator of Ageing Signals? Nat Rev Mol Cell Biol. 2007; 8:722-728. [PubMed: 17700625]

25. Nisimoto Y, Diebold BA, Constentino-Gomes D, Lambeth JD. Nox4: A Hydrogen PeroxideGenerating Oxygen Sensor. Biochemistry. 2014; 53:5111-5120. [PubMed: 25062272]

26. Wu RF, Ma Z, Liu Z, Terada LS. Nox4-Derived H2O2 Mediates Endoplasmic Reticulum Signaling through Local Ras Activation. Mol Cell Biol. 2010; 30:3553-3568. [PubMed: 20457808]

27. Kelley EE, Khoo NKH, Hundley NJ, Malik UZ, Freeman BA, Tarpey MM. Hydrogen Peroxide is the Major Oxidant Product of Xanthine Oxidase. Free Radical Biol Med. 2010; 48:493-498. [PubMed: 19941951]

28. Sies H. Biochemistry of the Peroxisome in the Liver Cell. Angew Chem Int Ed. 1974; 13:706-718. 
29. Sies H. Hydrogen Peroxide as a Central Redox Signaling Molecule in Physiological Oxidative Stress: Oxidative Eustress. Redox Biology. 2017; 11:613-619. [PubMed: 28110218]

30. Schreck R, Rieber P, Baeuerle PA. Reactive Oxygen Intermediates as Apparently Widely Used Messengers in the Activation of the NF-kappa B Transcription Factor and HIV-1. EMBO J. 1991; 10:2247-2258. [PubMed: 2065663]

31. Finkel T. Signal Transduction by Reactive Oxygen Species. J Cell Biol. 2011; 194:7-15. [PubMed: 21746850]

32. Veal EA, Day AM, Morgan BA. Hydrogen Peroxide Sensing and Signaling. Mol Cell. 2007; 26:114. [PubMed: 17434122]

33. Laing M. The Three Forms of Molecular Oxygen. J Chem Educ. 1989; 66:453.

34. Wood PM. The Potential Diagram for Oxygen at pH 7. Biochem J. 1988; 253:287-289. [PubMed: 2844170]

35. White EH, Wei CC. A Possible Role for Chemically-Produced Excited States in Biology. Biochem Biophys Res Commun. 1970; 39:1219-1223. [PubMed: 5513255]

36. Kanofsky JR. Singlet Oxygen Production by Biological Systems. Chem-Biol Interact. 1989; 70:128. [PubMed: 2472224]

37. Regensburger J, Knak A, Maisch T, Landthaler M, Bäumler W. Fatty Acids and Vitamins Generate Singlet Oxygen Under UVB Irradiation. Exp Dermatol. 2012; 21:135-139. [PubMed: 22229443]

38. Schweitzer C, Mehrdad Z, Noll A, Grabner EW, Schmidt R. Mechanism of Photosensitized Generation of Singlet Oxygen During Oxygen Quenching of Triplet States and the General Dependence of the Rate Constants and Efficiencies of O2(1 $\Sigma \mathrm{g}+), \mathrm{O} 2(1 \Delta \mathrm{g})$, and $\mathrm{O} 2(3 \Sigma \mathrm{g}-)$ Formation on Sensitizer Triplet State Energy and Oxidation Potential. J Phys Chem A. 2003; 107:2192-2198.

39. Koppenol WH, Kissner R. Can ONOOH Undergo Homolysis? Chem Res Toxicol. 1998; 11:87-90. [PubMed: 9511898]

40. Martinez GR, Di Mascio P, Bonini MG, Augusto O, Briviba K, Sies H, Maurer P, Röthlisberger U, Herold S, Koppenol WH. Peroxynitrite Does Not Decompose to Singlet Oxygen $(1) \Delta(\mathrm{g}) \mathrm{O}(2))$ and Nitroxyl (NO(-)). Proc Natl Acad Sci U S A. 2000; 97:10307-10312. [PubMed: 10973492]

41. Khan AU, Kovacic D, Kolbanovskiy A, Desai M, Frenkel K, Geacintov NE. The Decomposition of Peroxynitrite to Nitroxyl Anion (NO(-)) and Singlet Oxygen in Aqueous Solution. Proc Natl Acad Sci U S A. 2000; 97:2984-2989. [PubMed: 10716721]

42. Kochevar IE. Singlet Oxygen Signaling: From Intimate to Global. Sci STKE. 2004; 2004:pe7. [PubMed: 14983102]

43. Rahimtula A, O’brien PJ. The Possible Involvement of Singlet Oxygen in Prostaglandin Biosynthesis. Biochem Biophys Res Commun. 1976; 70:893-899. [PubMed: 820344]

44. Baier J, Maisch T, Maier M, Engel E, Landthaler M, Bäumler W. Singlet Oxygen Generation by UVA Light Exposure of Endogenous Photosensitizers. Biophys J. 2006; 91:1452-1459. [PubMed: 16751234]

45. Mütze S, Hebling U, Stremmel W, Wang J, Arnhold J, Pantopoulos K, Mueller S. Myeloperoxidase-Derived Hypochlorous Acid Antagonizes the Oxidative Stress-Mediated Activation of Iron Regulatory Protein 1. J Biol Chem. 2003; 278:40542-40549. [PubMed: 12888561]

46. Pullar J, Winterbourn C, Vissers M. Loss of GSH and Thiol Enzymes in Endothelial Cells Exposed to Sublethal Concentrations of Hypochlorous Acid. Am J Physiol. 1999; 277:H1505-1512. [PubMed: 10516189]

47. Pullar JM, Vissers MCM, Winterbourn CC. Living with a Killer: The Effects of Hypochlorous Acid on Mammalian Cells. IUBMB Life. 2000; 50:259-266. [PubMed: 11327319]

48. Hammerschmidt S, Wahn H. The Oxidants Hypochlorite and Hydrogen Peroxide Induce Distinct Patterns of Acute Lung Injury. Biochim Biophys Acta. 2004; 1690:258-264. [PubMed: 15511633]

49. Storkey C, Davies MJ, Pattison DI. Reevaluation of the Rate Constants for the Reaction of Hypochlorous Acid ( $\mathrm{HOCl})$ with Cysteine, Methionine, and Peptide Derivatives Using a New Competition Kinetic Approach. Free Radical Biol Med. 2014; 73:60-66. [PubMed: 24794410]

50. Morris JC. The Acid Ionization Constant of $\mathrm{HOCl}$ from 5 to $35^{\circ}$. J Phys Chem. 1966; 70:37983805. 
51. Peskin AV, Winterbourn CC. Kinetics of the Reactions of Hypochlorous Acid and Amino Acid Chloramines with Thiols, Methionine, and Ascorbate. Free Radical Biol Med. 2001; 30:572-579. [PubMed: 11182528]

52. Pullar JM, Vissers MCM, Winterbourn CC. Glutathione Oxidation by Hypochlorous Acid in Endothelial Cells Produces Glutathione Sulfonamide as a Major Product but not Glutathione Disulfide. J Biol Chem. 2001; 276:22120-22125. [PubMed: 11283008]

53. Fuchs PL, editorHandbook of Reagents for Organic Synthesis: Catalytic Oxidation Reagents. Wiley; 2013.

54. Winterbourn CC. Biological Reactivity and Biomarkers of the Neutrophil Oxidant, Hypochlorous Acid. Toxicology. 2002; 181-182:223-227.

55. Pattison DI, Hawkins CL, Davies MJ. Hypochlorous Acid-Mediated Protein Oxidation:w How Important are Chloramine Transfer Reactions and Protein Tertiary Structure? Biochemistry. 2007; 46:9853-9864. [PubMed: 17676767]

56. Afshinnia F, Zeng L, Byun J, Gadegbeku CA, Magnone MC, Whatling C, Valastro B, Kretzler M, Pennathur S. Myeloperoxidase Levels and Its Product 3-Chlorotyrosine Predict Chronic Kidney Disease Severity and Associated Coronary Artery Disease. Am J Nephrol. 2017; 46:73-81. [PubMed: 28668952]

57. Schieven GL, Fex HD, Stephenson L. Hypochlorous Acid Activates Tyrosine Phosphorylation Signal Pathways Leading to Calcium Signaling and TNFa Production. Antioxid Redox Signal. 2002; 4:501-507. [PubMed: 12215218]

58. Halliwell B, Gutteridge JMC. Free Radicals in Biology and Medicine. 2. Oxford University Press; Oxford: 1999.

59. Mikkelsen RB, Wardman P. Biological Chemistry of Reactive Oxygen and Nitrogen and RadiationInduced Signal Transduction Mechanisms. Oncogene. 2003; 22:5734-5754. [PubMed: 12947383]

60. Giorgio M, Trinei M, Migliaccio E, Pelicci PG. Hydrogen peroxide: a metabolic by-product or a common mediator of ageing signals? Nature Reviews Molecular Cell Biology. 2007; 8:722. [PubMed: 17700625]

61. Winterbourn CC. Revisiting the Reactions of Superoxide with Glutathione and Other Thiols. Arch Biochem Biophys. 2016; 595:68-71. [PubMed: 27095219]

62. Jones CM, Lawrence A, Wardman P, Burkitt MJ. Electron Paramagnetic Resonance Spin Trapping Investigation into the Kinetics of Glutathione Oxidation by the Superoxide Radical: Re-Evaluation of the Rate Constant. Free Radical Biol Med. 2002; 32:982-990. [PubMed: 12008114]

63. Aruoma OI, Halliwell B, Hoey BM, Butler J. The Antioxidant Action of N-Acetylcysteine: Its Reaction with Hydrogen Peroxide, Hydroxyl Radical, Superoxide, and Hypochlorous Acid. Free Radical Biol Med. 1989; 6:593-597. [PubMed: 2546864]

64. Antunes F, Cadenas E. Estimation of H2O2 Gradients Across Biomembranes. FEBS Lett. 2000; 475:121-126. [PubMed: 10858501]

65. Lim JB, Huang BK, Deen WM, Sikes HD. Analysis of the Lifetime and Spatial Localization of Hydrogen Peroxide Generated in the Cytosol Using a Reduced Kinetic Model. Free Radical Biol Med. 2015; 89:47-53. [PubMed: 26169725]

66. Marinho HS, Real C, Cyrne L, Soares H, Antunes F. Hydrogen Peroxide Sensing, Signaling and Regulation of Transcription Factors. Redox Biol. 2014; 2:535-562. [PubMed: 24634836]

67. Roots R, Okada S. Estimation of Life Times and Diffusion Distances of Radicals Involved in Xray-Induced DNA Strand Breaks or Killing of Mammalian Cells. Radiat Res. 1975; 64:306-320. [PubMed: 1197641]

68. Mezyk SP. Rate Constant Determination for the Reaction of Hydroxyl and Glutathione Thiyl Radicals with Glutathione in Aqueous Solution. J Phys Chem A. 1996; 100:8861-8866.

69. Halliwell B. How to Characterize an Antioxidant: An Update. Biochem Soc Symp. 1995; 61:73101. [PubMed: 8660405]

70. Nalwaya N, Deen WM. Analysis of Cellular Exposure to Peroxynitrite in Suspension Cultures. Chem Res Toxicol. 2003; 16:920-932. [PubMed: 12870895]

71. Szabo C, Ischiropoulos H, Radi R. Peroxynitrite: Biochemistry, Pathophysiology and Development of Therapeutics. Nat Rev Drug Discov. 2007; 6:662-680. [PubMed: 17667957] 
72. Radi R. Peroxynitrite, a Stealthy Biological Oxidant. J Biol Chem. 2013; 288:26464-26472. [PubMed: 23861390]

73. Ferrer-Sueta G, Campolo N, Trujillo M, Bartesaghi S, Carballal S, Romero N, Alvarez B, Radi R. Biochemistry of Peroxynitrite and Protein Tyrosine Nitration. Chem Rev. 2018; 118:1338-1408. [PubMed: 29400454]

74. Rodgers MaJSnowden PT. Lifetime of Oxygen in Liquid Water as Determined by Time-Resolved Infrared Luminescence Measurements. J Am Chem Soc. 1982; 104:5541-5543.

75. Wilkinson F, Helman WP, Ross AB. Rate Constants for the Decay and Reactions of the Lowest Electronically Excited Singlet State of Molecular Oxygen in Solution. An Expanded and Revised Compilation. J Phys Chem Ref Data. 1995; 24:663.

76. Redmond RW, Kochevar IE. Symposium-in-Print: Singlet Oxygen Invited Review. Photochem Photobiol. 2006; 82:1178-1186. [PubMed: 16740059]

77. Baier J, Maier M, Engl R, Landthaler M, Bäumler W. Time-Resolved Investigations of Singlet Oxygen Luminescence in Water, in Phosphatidylcholine, and in Aqueous Suspensions of Phosphatidylcholine or HT29 Cells. J Phys Chem B. 2005; 109:3041-3046. [PubMed: 16851318]

78. Snyder JW, Skovsen E, Lambert JDC, Ogilby PR. Subcellular, Time-Resolved Studies of Singlet Oxygen in Single Cells. J Am Chem Soc. 2005; 127:14558-14559. [PubMed: 16231893]

79. Gorman AA, Rodgers MA. Current Perspectives of Singlet Oxygen Detection in Biological Environments. J Photochem Photobiol B: Biol. 1992; 14:159-176.

80. Sies H, Menck CFM. Singlet Oxygen Induced DNA Damage. Mutat Res. 1992; 275:367-375. [PubMed: 1383777]

81. Baier J, Maier M, Engl R, Landthaler M, Baumler W. Time-Resolved Investigations of Singlet Oxygen Luminescence in Water, in Phosphatidylcholine, and in Aqueous Suspensions of Phosphatidylcholine or HT29 Cells. J Phys Chem B. 1992; 109:3041-3046.

82. Devasagayam TPA, Sundquist AR, Di Mascio P, Kaiser S, Sies H. Activity of Thiols as Singlet Molecular Oxygen Quenchers. J Photochem Photobio, B. 1991; 9:105-116.

83. Winterbourn CC, Hampton MB, Livesey JH, Kettle AJ. Modeling the Reactions of Superoxide and Myeloperoxidase in the Neutrophil Phagosome: Implications for Microbial Killing. J Biol Chem. 2006; 281:39860-39869. [PubMed: 17074761]

84. Yang Y, Sharma A, Sharma R, Patrick B, Singhal SS, Zimniak P, Awasthi S, Awasthi YC. Cells Preconditioned with Mild, Transient UVA Irradiation Acquire Resistance to Oxidative Stress and UVA-Induced Apoptosis: Role of 4-Hydroxynonenal in UVA-Mediated Signaling for Apoptosis. J Biol Chem. 2003; 278:41380-41388. [PubMed: 12888579]

85. Esterbauer H, Schaur RJ, Zollner H. Chemistry and Biochemistry of 4-Hydroxynonenal, Malonaldehyde and Related Aldehydes. Free Radical Biol Med. 1991; 11:81-128. [PubMed: 1937131]

86. Selley ML, Bartlett MR, Mcguiness JA, Hapel AJ, Ardlie NG. Determination of the Lipid Peroxidation Product Trans-4-Hydroxy-2-Nonenal in Biological Samples by High-Performance Liquid Chromatography and Combined Capillary Column Gas Chromatography-Negative-Ion Chemical Ionisation Mass Spectrometry. J Chromatogr. 1989; 488:329-340. [PubMed: 2745626]

87. Chen ZH, Niki E. 4-Hydroxynonenal (4-HNE) has Been Widely Accepted as an Inducer of Oxidative Stress. Is this the Whole Truth About it or Can 4-HNE also Exert Protective Effects? IUBMB Life. 2006; 58:372-373. [PubMed: 16754333]

88. Siems WG, Zollner H, Grune T, Esterbauer H. Metabolic Fate of 4-Hydroxynonenal in Hepatocytes: 1,4-Dihydroxynonene is not the Main Product. J Lipid Res. 1997; 38:612-622. [PubMed: 9101442]

89. Vazdar M, Jurkiewicz P, Hof M, Jungwirth P, Cwiklik L. Behavior of 4-Hydroxynonenal in Phospholipid Membranes. J Phys Chem B. 2012; 116:6411-6415. [PubMed: 22577896]

90. Doorn JA, Petersen DR. Covalent Modification of Amino Acid Nucleophiles by the Lipid Peroxidation Products 4-Hydroxy-2-nonenal and 4-Oxo-2-nonenal. Chem Res Toxicol. 2002; 15:1445-1450. [PubMed: 12437335]

91. Baker PRS, Lin Y, Schopfer FJ, Woodcock SR, Groeger AL, Batthyany C, Sweeney S, Long MH, Iles KE, Baker LMS, et al. Fatty Acid Transduction of Nitric Oxide Signaling: Multiple Nitrated Unsaturated Fatty Acid Derivatives Exist in Human Blood and Urine and Serve as Endogenous 
Peroxisome Proliferator-Activated Receptor Ligands. J Biol Chem. 2005; 280:42464-42475. [PubMed: 16227625]

92. Salvatore SR, Vitturi DA, Baker PR, Bonacci G, Koenitzer JR, Woodcock SR, Freeman BA, Schopfer FJ. Characterization and Quantification of Endogenous Fatty Acid Nitroalkene Metabolites in Human Urine. J Lipid Res. 2013; 54:1998-2009. [PubMed: 23620137]

93. Delmastro-Greenwood M, Hughan KS, Vitturi DA, Salvatore SR, Grimes G, Potti G, Shiva S, Schopfer FJ, Gladwin MT, Freeman BA, et al. Nitrite and Nitrate-Dependent Generation of AntiInflammatory Fatty Acid Nitroalkenes. Free Radical Biol Med. 2015; 89:333-341. [PubMed: 26385079]

94. Rudolph V, Rudolph TK, Schopfer FJ, Bonacci G, Woodcock SR, Cole MP, Baker PRS, Ramani R, Freeman BA. Endogenous Generation and Protective Effects of Nitro-Fatty Acids in a Murine Model of Focal Cardiac Ischaemia and Reperfusion. Cardiovasc Res. 2010; 85:155-166. [PubMed: 19666678]

95. Baker LMS, Baker PRS, Golin-Bisello F, Schopfer FJ, Fink M, Woodcock SR, Branchaud BP, Radi R, Freeman BA. Nitro-Fatty Acid Reaction with Glutathione and Cysteine: Kinetic Analysis of Thiol Alkylation by a Michael Addition Reaction. J Biol Chem. 2007; 282:31085-31093. [PubMed: 17720974]

96. Bell-Parikh LC, Ide T, Lawson JA, Mcnamara P, Reilly M, Fitzgerald GA. Biosynthesis of 15deoxy- $\Delta(12,14)-P G(2)$ and the Ligation of PPAR $\gamma$. J Clin Invest. 2003; 112:945-955. [PubMed: 12975479]

97. Owen JB, Butterfield DA. Protein Misfolding and Cellular Stress in Disease and Aging: Concepts and Protocols. Bross P, Gregersen N, editorsHumana Press; Totowa, NJ: 2010.

98. Chakravarthi S, Jessop CE, Bulleid NJ. The Role of Glutathione in Disulphide Bond Formation and Endoplasmic-Reticulum-Generated Oxidative Stress. EMBO rep. 2006; 7:271-275. [PubMed: 16607396]

99. Crush KG. Carnosine and Related Substances in Animal Tissues. Comp Biochem Physiol. 1970; 34:3-30. [PubMed: 4988625]

100. Barski OA, Xie Z, Baba SP, Sithu SD, Agarwal A, Cai J, Bhatnagar A, Srivastava S. Dietary Carnosine Prevents Early Atherosclerotic Lesion Formation in Apolipoprotein E-Null Mice. Arterioscler, Thromb, Vasc Biol. 2013; 33:1162-1170. [PubMed: 23559625]

101. Tyler DD. Polarographic assay and intracellular distribution of superoxide dismutase in rat liver. Biochemical Journal. 1975; 147:493-504. [PubMed: 810138]

102. Weydert CJ, Cullen JJ. Measurement of Superoxide Dismutase, Catalase, and Glutathione Peroxidase in Cultured Cells and Tissue. Nat Protoc. 2010; 5:51-66. [PubMed: 20057381]

103. Gray B, Carmichael AJ. Kinetics of Superoxide Scavenging by Dismutase Enzymes and Manganese Mimics Determined by Electron Spin Resonance. Biochem J. 1992; 281:795-802. [PubMed: 1311175]

104. Ranguelova K, Ganini D, Bonini MG, London RE, Mason RP. Kinetics of the Oxidation of Reduced Cu,Zn-Superoxide Dismutase by Peroxymonocarbonate. Free Radical Biol Med. 2012; 53:589-594. [PubMed: 22569304]

105. Cox AG, Pearson AG, Pullar JM, Jönsson TJ, Lowther WT, Winterbourn CC, Hampton MB. Mitochondrial Peroxiredoxin 3 is More Resilient to Hyperoxidation than Cytoplasmic Peroxiredoxins. Biochem J. 2009; 421:51-58. [PubMed: 19356151]

106. Trujillo M, Ferrer-Sueta G, Radi R. Kinetic Studies on Peroxynitrite Reduction by Peroxiredoxins. Methods Enzymol. 2008; 441:173-196. [PubMed: 18554535]

107. Manta B, Hugo M, Ortiz C, Ferrer-Sueta G, Trujillo M, Denicola A. The Peroxidase and Peroxynitrite Reductase Activity of Human Erythrocyte Peroxiredoxin 2. Arch Biochem Biophys. 2009; 484:146-154. [PubMed: 19061854]

108. Wood ZA, Schröder E, Robin Harris J, Poole LB. Structure, Mechanism and Regulation of Peroxiredoxins. Trends Biochem Sci. 2003; 28:32-40. [PubMed: 12517450]

109. Peskin AV, Low FM, Paton LN, Maghzal GJ, Hampton MB, Winterbourn CC. The High Reactivity of Peroxiredoxin 2 with $\mathrm{H} 2 \mathrm{O} 2$ is not Reflected in its Reaction with Other Oxidants and Thiol Reagents. J Biol Chem. 2007; 282:11885-11892. [PubMed: 17329258] 
110. Perkins A, Nelson KJ, Parsonage D, Poole LB, Karplus PA. Peroxiredoxins: Guardians Against Oxidative Stress and Modulators of Peroxide Signaling. Trends Biochem Sci. 2015; 40:435-445. [PubMed: 26067716]

111. Winterbourn CC. The Biological Chemistry of Hydrogen Peroxide. Methods Enzymol. 2013; 528:3-25. [PubMed: 23849856]

112. Winterbourn CC. Biological Production, Detection, and Fate of Hydrogen Peroxide. Antioxid Redox Signal. 2017; 29:541-551. [PubMed: 29113458]

113. Imamura H, Huynh Nhat KP, Togawa H, Saito K, Iino R, Kato-Yamada Y, Nagai T, Noji H. Visualization of ATP Levels Inside Single Living Cells with Fluorescence Resonance Energy Transfer-Based Genetically Encoded Indicators. Proc Natl Acad Sci U S A. 2009; 106:1565115656. [PubMed: 19720993]

114. Sims JJ, Scavone F, Cooper EM, Kane LA, Youle RJ, Boeke JD, Cohen RE. Polyubiquitin-Sensor Proteins Reveal Localization and Linkage-Type Dependence of Cellular Ubiquitin Signaling. Nat Methods. 2012; 9:303-309. [PubMed: 22306808]

115. Schopfer FJ, Cipollina C, Freeman BA. Formation and Signaling Actions of Electrophilic Lipids. Chem Rev. 2011; 111:5997-6021. [PubMed: 21928855]

116. Winterbourn CC. Reconciling the Chemistry and Biology of Reactive Oxygen Species. Nat Chem Biol. 2008; 4:278-286. [PubMed: 18421291]

117. Stöcker S, Van Laer K, Mijuskovic A, Dick TP. The Conundrum of Hydrogen Peroxide Signaling and the Emerging Role of Peroxiredoxins as Redox Relay Hubs. Antioxid Redox Signal. 2018; 28:558-573. [PubMed: 28587525]

118. Fritz R, Bol J, Hebling U, Angermüller S, Völkl A, Fahimi HD, Mueller S. CompartmentDependent Management of H2O2 by Peroxisomes. Free Radical Biol Med. 2007; 42:1119-1129. [PubMed: 17349938]

119. Bedard K, Krause KH. The NOX Family of ROS-Generating NADPH Oxidases: Physiology and Pathophysiology. Physiol Rev. 2007; 87:245-313. [PubMed: 17237347]

120. Henderson LM, Meech RW. Evidence That the Product of the Human X-linked Cgd Gene, Gp91phox, is a Voltage-Gated H(+) Pathway. J Gen Physiol. 1999; 114:771-786. [PubMed: 10578014]

121. Lambeth JD. NOX Enzymes and the Biology of Reactive Oxygen. Nat Rev Immunol. 2004; 4:181-189. [PubMed: 15039755]

122. Suh YA, Arnold RS, Lassegue B, Shi J, Xu X, Sorescu D, Chung AB, Griendling KK, Lambeth JD. Cell Transformation by the Superoxide-Generating Oxidase Mox1. Nature. 1999; 401:79-82. [PubMed: 10485709]

123. Geiszt M, Kopp JB, Várnai P, Leto TL. Identification of Renox, an NAD(P)H Oxidase in Kidney. Proc Natl Acad Sci U S A. 2000; 97:8010-8014. [PubMed: 10869423]

124. Shiose A, Kuroda J, Tsuruya K, Hirai M, Hirakata H, Naito S, Hattori M, Sakaki Y, Sumimoto H. A Novel Superoxide-Producing NAD(P)H Oxidase in Kidney. J Biol Chem. 2001; 276:14171423. [PubMed: 11032835]

125. Bánfi B, Molnár G, Maturana A, Steger K, Hegedûs B, Demaurex N, Krause KH. A Ca2+Activated NADPH Oxidase In Testis, Spleen, And Lymph Nodes. J Biol Chem. 2001; 276:37594-37601. [PubMed: 11483596]

126. De Deken X, Wang D, Many MC, Costagliola S, Libert F, Vassart G, Dumont JE, Miot F. Cloning of Two Human Thyroid cDNAs Encoding New Members of the NADPH Oxidase Family. J Biol Chem. 2000; 275:23227-23233. [PubMed: 10806195]

127. Ambasta RK, Kumar P, Griendling KK, Schmidt HHHW, Busse R, Brandes RP. Direct Interaction of the Novel Nox Proteins with p22phox is Required for the Formation of a Functionally Active NADPH Oxidase. J Biol Chem. 2004; 279:45935-45941. [PubMed: 15322091]

128. Kuroda J, Nakagawa K, Yamasaki T, Nakamura K-I, Takeya R, Kuribayashi F, Imajoh-Ohmi S, Igarashi K, Shibata Y, Sueishi K, et al. The Superoxide-Producing NAD(P)H Oxidase Nox4 in the Nucleus of Human Vascular Endothelial Cells. Genes Cells. 2005; 10:1139-1151. [PubMed: 16324151]

129. Chamulitrat W, Schmidt R, Tomakidi P, Stremmel W, Chunglok W, Kawahara T, Rokutan K. Association of gp91phox Homolog Nox1 with Anchorage-Independent Growth and Map Kinase- 
Activation of Transformed Human Keratinocytes. Oncogene. 2003; 22:6045-6053. [PubMed: 12955083]

130. Block K, Gorin Y, Abboud HE. Subcellular Localization of Nox4 and Regulation in Diabetes. Proc Natl Acad Sci U S A. 2009; 106:14385-14390. [PubMed: 19706525]

131. Brown DI, Griendling KK. Nox Proteins in Signal Transduction. Free Radical Biol Med. 2009; 47:1239-1253. [PubMed: 19628035]

132. Kawahara T, Lambeth JD. Phosphatidylinositol (4,5)-Bisphosphate Modulates Nox5 Localization via an N-Terminal Polybasic Region. Mol Biol Cell. 2008; 19:4020-4031. [PubMed: 18614798]

133. Murphy Michael p. How Mitochondria Produce Reactive Oxygen Species. Biochem J. 2009; 417:1-13. [PubMed: 19061483]

134. Chance B, Sies H, Boveris A. Hydroperoxide Metabolism in Mammalian Organs. Physiol Rev. 1979; 59:527-605. [PubMed: 37532]

135. St-Pierre J, Buckingham JA, Roebuck SJ, Brand MD. Topology of Superoxide Production from Different Sites in the Mitochondrial Electron Transport Chain. J Biol Chem. 2002; 277:4478444790. [PubMed: 12237311]

136. Andreyev AY, Kushnareva YE, Starkov AA. Mitochondrial Metabolism of Reactive Oxygen Species. Biochemistry (Moscow). 2005; 70:200-214. [PubMed: 15807660]

137. Venditti P, Di Stefano L, Di Meo S. Mitochondrial Metabolism of Reactive Oxygen Species. Mitochondrion. 2013; 13:71-82. [PubMed: 23376030]

138. Lebovitz RM, Zhang H, Vogel H, Cartwright J, Dionne L, Lu N, Huang S, Matzuk MM. Neurodegeneration, Myocardial Injury, and Perinatal Death in Mitochondrial Superoxide Dismutase-Deficient Mice. Proc Natl Acad Sci U S A. 1996; 93:9782-9787. [PubMed: 8790408]

139. Kinoshita M, Sakamoto T, Kashio A, Shimizu T, Yamasoba T. Age-Related Hearing Loss in MnSOD Heterozygous Knockout Mice. Oxid Med Cell Longev. 2013; 2013:12.

140. Sentman ML, Granström M, Jakobson H, Reaume A, Basu S, Marklund SL. Phenotypes of Mice Lacking Extracellular Superoxide Dismutase and Copper- and Zinc-Containing Superoxide Dismutase. J Biol Chem. 2006; 281:6904-6909. [PubMed: 16377630]

141. Quinlan CL, Perevoshchikova IV, Hey-Mogensen M, Orr AL, Brand MD. Sites of Reactive Oxygen Species Generation by Mitochondria Oxidizing Different Substrates. Redox Biolology. 2013; 1:304-312.

142. Orr AL, Quinlan CL, Perevoshchikova IV, Brand MD. A Refined Analysis of Superoxide Production by Mitochondrial Sn-Glycerol 3-Phosphate Dehydrogenase. J Biol Chem. 2012; 287:42921-42935. [PubMed: 23124204]

143. Sazanov LA. A Giant Molecular Proton Pump: Structure and Mechanism of Respiratory Complex I. Nat Rev Mol Cell Biol. 2015; 16:375-388. [PubMed: 25991374]

144. Dworakowski R, Anilkumar N, Zhang M, Shah AM. Redox Signalling Involving NADPH Oxidase-Derived Reactive Oxygen Species. Biochem Soc Trans. 2006; 34:960-964. [PubMed: 17052237]

145. Mccann S, Roulston C. NADPH Oxidase as a Therapeutic Target for Neuroprotection against Ischaemic Stroke: Future Perspectives. Brain Sci. 2013; 3:561. [PubMed: 24961415]

146. Zangar RC, Davydov DR, Verma S. Mechanisms that Regulate Production of Reactive Oxygen Species by Cytochrome P450. Toxicol Appl Pharmacol. 2004; 199:316-331. [PubMed: 15364547]

147. Boveris A. Methods Enzymol. Vol. 105. Academic Press; 1984.

148. Kussmaul L, Hirst J. The Mechanism of Superoxide Production by NADH:Ubiquinone Oxidoreductase (Complex I) from Bovine Heart Mitochondria. Proc Natl Acad Sci U S A. 2006; 103:7607-7612. [PubMed: 16682634]

149. Romero E, Gómez Castellanos JR, Gadda G, Fraaije MW, Mattevi A. Same Substrate, Many Reactions: Oxygen Activation in Flavoenzymes. Chem Rev. 2018; 118:1742-1769. [PubMed: 29323892]

150. Korshunov SS, Skulachev VP, Starkov AA. High Protonic Potential Actuates a Mechanism of Production of Reactive Oxygen Species in Mitochondria. FEBS Lett. 1997; 416:15-18. [PubMed: 9369223] 
151. Adam-Vizi V, Chinopoulos C. Bioenergetics and the Formation of Mitochondrial Reactive Oxygen Species. Trends in Pharmacol Sci. 2006; 27:639-645. [PubMed: 17056127]

152. Turrens JF, Alexandre A, Lehninger AL. Ubisemiquinone is the Electron Donor for Superoxide Formation by Complex Iii of Heart Mitochondria. Arch Biochem Biophys. 1985; 237:408-414. [PubMed: 2983613]

153. Muller FL, Liu Y, Van Remmen H. Complex III Releases Superoxide to Both Sides of the Inner Mitochondrial Membrane. J Biol Chem. 2004; 279:49064-49073. [PubMed: 15317809]

154. Sena LA, Chandel NS. Physiological Roles of Mitochondrial Reactive Oxygen Species. Mol Cell. 2012; 48:158-167. [PubMed: 23102266]

155. Starkov AA, Fiskum G, Chinopoulos C, Lorenzo BJ, Browne SE, Patel MS, Beal MF. Mitochondrial a-Ketoglutarate Dehydrogenase Complex Generates Reactive Oxygen Species. J Neurosci. 2004; 24:7779-7788. [PubMed: 15356189]

156. Giorgio M, Migliaccio E, Orsini F, Paolucci D, Moroni M, Contursi C, Pelliccia G, Luzi L, Minucci S, Marcaccio M, et al. Electron Transfer Between Cytochrome C and p66Shc Generates Reactive Oxygen Species that Trigger Mitochondrial Apoptosis. Cell. 2005; 122:221-233. [PubMed: 16051147]

157. Go YM, Jones DP. Redox Compartmentalization in Eukaryotic Cells. Biochim Biophys Acta. 2008; 1780:1273-1290. [PubMed: 18267127]

158. Tu BP, Weissman JS. Oxidative Protein Folding in Eukaryotes: Mechanisms and Consequences. J Cell Biol. 2004; 164:341-346. [PubMed: 14757749]

159. Bulleid NJ, Ellgaard L. Multiple Ways to Make Disulfides. Trends Biochem Sci. 2011; 36:485492. [PubMed: 21778060]

160. Freedman RB. Protein Disulfide Isomerase: Multiple Roles in the Modification of Nascent Secretory Proteins. Cell. 1989; 57:1069-1072. [PubMed: 2544299]

161. Satoh M, Shimada A, Kashiwai A, Saga S, Hosokawa M. Differential Cooperative Enzymatic Activities of Protein Disulfide Isomerase Family in Protein Folding. Cell Stress Chaperones. 2005; 10:211-220. [PubMed: 16184766]

162. Mazzarella RA, Srinivasan M, Haugejorden SM, Green M. ERp72, an Abundant Luminal Endoplasmic Reticulum Protein, Contains Three Copies of the Active Site Sequences of Protein Disulfide Isomerase. J Biol Chem. 1990; 265:1094-1101. [PubMed: 2295602]

163. Braakman I, Bulleid NJ. Protein Folding and Modification in the Mammalian Endoplasmic Reticulum. Annu Rev Biochem. 2011; 80:71-99. [PubMed: 21495850]

164. Kosuri P, Alegre-Cebollada J, Feng J, Kaplan A, Inglés-Prieto A, Badilla Carmen 1, Stockwell Brent r, Sanchez-Ruiz Jose m, Holmgren A, Fernández Julio m. Protein Folding Drives Disulfide Formation. Cell. 2012; 151:794-806. [PubMed: 23141538]

165. Hudson DA, Gannon SA, Thorpe C. Oxidative Protein Folding: From Thiol-Disulfide Exchange Reactions to the Redox Poise of the Endoplasmic Reticulum. Free Radical Biol Med. 2015; $0: 171-182$.

166. Tu BP, Ho-Schleyer SC, Travers KJ, Weissman JS. Biochemical Basis of Oxidative Protein Folding in the Endoplasmic Reticulum. Science. 2000; 290:1571-1574. [PubMed: 11090354]

167. Tu BP, Weissman JS. The FAD- and $\mathrm{O}_{2}$-Dependent Reaction Cycle of Ero1-Mediated Oxidative Protein Folding in the Endoplasmic Reticulum. Mol Cell. 2002; 10:983-994. [PubMed: 12453408]

168. Zeeshan HMA, Lee GH, Kim HR, Chae HJ. Endoplasmic Reticulum Stress and Associated ROS. Int J Mol Sci. 2016; 17:327. [PubMed: 26950115]

169. Margittai É, Enyedi B, Csala M, Geiszt M, Bánhegyi G. Composition of the Redox Environment of the Endoplasmic Reticulum and Sources of Hydrogen Peroxide. Free Radical Biol Med. 2015; 83:331-340. [PubMed: 25678412]

170. Kodali VK, Thorpe C. Oxidative Protein Folding and the Quiescin-Sulfhydryl Oxidase Family of Flavoproteins. Antioxid Redox Signal. 2010; 13:1217-1230. [PubMed: 20136510]

171. Poulsen LL, Ziegler DM. The Liver Microsomal FAD-Containing Monooxygenase. Spectral Characterization and Kinetic Studies. J Biol Chem. 1979; 254:6449-6455. [PubMed: 36396]

172. Davydov DR. Microsomal Monooxygenase in Apoptosis: Another Target for Cytochrome C Signaling? Trends Biochem Sci. 2001; 26:155-160. [PubMed: 11246020] 
173. Harrington EA, Bebbington D, Moore J, Rasmussen RK, Ajose-Adeogun AO, Nakayama T, Graham JA, Demur C, Hercend T, Diu-Hercend A, et al. VX-680, a Potent and Selective SmallMolecule Inhibitor of the Aurora Kinases, Suppresses Tumor Growth in Vivo. Nat Med. 2004; 10:262-267. [PubMed: 14981513]

174. Catucci G, Occhipinti A, Maffei M, Gilardi G, Sadeghi S. Effect of Human Flavin-Containing Monooxygenase 3 Polymorphism on the Metabolism of Aurora Kinase Inhibitors. Int J Mol Sci. 2013; 14:2707. [PubMed: 23358255]

175. Gruenke LD, Konopka K, Cadieu M, Waskell L. The Stoichiometry of the Cytochrome P-450Catalyzed Metabolism of Methoxyflurane and Benzphetamine in the Presence and Absence of Cytochrome b5. J Biol Chem. 1995; 270:24707-24718. [PubMed: 7559586]

176. Santos CXC, Nabeebaccus AA, Shah AM, Camargo LL, Filho SV, Lopes LR. Endoplasmic Reticulum Stress and Nox-Mediated Reactive Oxygen Species Signaling in the Peripheral Vasculature: Potential Role in Hypertension. Antioxid Redox Signal. 2014; 20:121-134. [PubMed: 23472786]

177. Bulleid Neil jVan Lith M. Redox Regulation in the Endoplasmic Reticulum. Biochem Soc Trans. 2014; 42:905-908. [PubMed: 25109977]

178. Ponsero AJ, Igbaria A, Darch MA, Miled S, Outten CE, Winther JR, Palais G, D'autréaux B, Delaunay-Moisan A, Toledano MB. Endoplasmic Reticulum Transport of Glutathione by Sec61 is Regulated by Ero1 And BiP. Mol Cell. 2017; 67:962-973. [PubMed: 28918898]

179. Bánhegyi G, Lusini L, Puskás F, Rossi R, Fulceri R, Braun L, Mile V, Di Simplicio P, Mandl J, Benedetti A. Preferential Transport of Glutathione Versus Glutathione Disulfide in Rat Liver Microsomal Vesicles. J Biol Chem. 1999; 274:12213-12216. [PubMed: 10212186]

180. Bánhegyi G, Csala M, Nagy G, Sorrentino V, Fulceri R, Benedetti A. Evidence for the Transport of Glutathione Through Ryanodine Receptor Channel Type 1. Biochem J. 2003; 376:807-812. [PubMed: 14519101]

181. Le Gall S, Neuhof A, Rapoport T. The Endoplasmic Reticulum Membrane is Permeable to Small Molecules. Mol Biol Cell. 2004; 15:447-455. [PubMed: 14617815]

182. Cuozzo JW, Kaiser CA. Competition Between Glutathione and Protein Thiols for DisulphideBond Formation. Nat Cell Biol. 1999; 1:130-135. [PubMed: 10559898]

183. Bass R, Ruddock LW, Klappa P, Freedman RB. A Major Fraction of Endoplasmic ReticulumLocated Glutathione is Present as Mixed Disulfides with Protein. J Biol Chem. 2004; 279:52575262. [PubMed: 14630926]

184. Bosello-Travain V, Conrad M, Cozza G, Negro A, Quartesan S, Rossetto M, Roveri A, Toppo S, Ursini F, Zaccarin M, et al. Protein Disulfide Isomerase and Glutathione are Alternative Substrates in the One Cys Catalytic Cycle of Glutathione Peroxidase 7. Biochim Biophys Acta. 2013; 1830:3846-3857. [PubMed: 23454490]

185. Nguyen VD, Saaranen MJ, Karala AR, Lappi AK, Wang L, Raykhel IB, Alanen HI, Salo KEH, Wang CC, Ruddock LW. Two Endoplasmic Reticulum PDI Peroxidases Increase the Efficiency of the Use of Peroxide During Disulfide Bond Formation. J Mol Biol. 2011; 406:503-515. [PubMed: 21215271]

186. Marí M, Morales A, Colell A, García-Ruiz C, Fernández-Checa JC. Mitochondrial Glutathione, a Key Survival Antioxidant. Antioxid Redox Signal. 2009; 11:2685-2700. [PubMed: 19558212]

187. Go YM, Jones DP. Redox compartmentalization in eukaryotic cells. Biochimica et Biophysica Acta (BBA) - General Subjects. 2008; 1780:1273-1290. [PubMed: 18267127]

188. Marí M, Morales A, Colell A, García-Ruiz C, Fernández-Checa JC. Mitochondrial Glutathione, a Key Survival Antioxidant. Antioxidants \& Redox Signaling. 2009; 11:2685-2700. [PubMed: 19558212]

189. Booty LM, King MS, Thangaratnarajah C, Majd H, James AM, Kunji ERS, Murphy MP. The Mitochondrial Dicarboxylate and 2-Oxoglutarate Carriers do not Transport Glutathione. FEBS Lett. 2015; 589:621-628. [PubMed: 25637873]

190. Bonekamp NA, Volkl A, Fahimi HD, Schrader M. Reactive Oxygen Species and Peroxisomes: Struggling for Balance. BioFactors. 2009; 35:346-355. [PubMed: 19459143]

191. Boveris A, Oshino N, Chance B. The Cellular Production of Hydrogen Peroxide. Biochem J. 1972; 128:617-630. [PubMed: 4404507] 
192. Rokka A, Antonenkov VD, Soininen R, Immonen HL, Pirilä PL, Bergmann U, Sormunen RT, Weckström M, Benz R, Hiltunen JK. Pxmp2 is a Channel-Forming Protein in Mammalian Peroxisomal Membrane. PLoS One. 2009; 4:e5090. [PubMed: 19352492]

193. Fransen M, Nordgren M, Wang B, Apanasets O. Role of Peroxisomes in ROS/RNS-metabolism: Implications for Human Disease. Biochim Biophys Acta. 2012; 1822:1363-1373. [PubMed: 22178243]

194. Nordgren M, Fransen M. Peroxisomal Metabolism and Oxidative Stress. Biochimie. 2014; 98:5662. [PubMed: 23933092]

195. Li Y, Tharappel JC, Cooper S, Glenn M, Glauert HP, Spear BT. Expression of the Hydrogen Peroxide-Generating Enzyme Fatty Acyl CoA Oxidase Activates NF-kappa B. DNA Cell Biol. 2000; 19:113-120. [PubMed: 10701777]

196. Heck DE, Vetrano AM, Mariano TM, Laskin JD. UVB light stimulates production of reactive oxygen species: unexpected role for catalyase. J Biol Chem. 2003; 278:22432-22436. [PubMed: 12730222]

197. Delcambre S, Nonnenmacher Y, Hiller K. Mitochondrial Mechanisms of Degeneration and Repair in Parkinson's Disease. Buhlman LM, editorSpringer International Publishing; Cham: 2016.

198. Li Y, Trush MA, Yager JD. DNA Damage Caused by Reactive Oxygen Species Originating from a Copper-Dependent Oxidation of the 2-Hydroxy Catechol of Estradiol. Carcinogenesis. 1994; 15:1421-1427. [PubMed: 8033320]

199. Onyango AN. Endogenous Generation of Singlet Oxygen and Ozone in Human and Animal Tissues: Mechanisms, Biological Significance, and Influence of Dietary Components. Oxid Med Cell Longev. 2016; 2016:1-22.

200. Penning TM. Genotoxicity of Ortho-quinones: Reactive Oxygen Species versus Covalent Modification. Toxicol Res. 2017; 6:740-754.

201. Sheng Y, Abreu IA, Cabelli DE, Maroney MJ, Miller AF, Teixeira M, Valentine JS. Superoxide Dismutases and Superoxide Reductases. Chem Rev. 2014; 114:3854-3918. [PubMed: 24684599]

202. Fridovich I. Superoxide Radical: An Endogenous Toxicant. Annu Rev Pharmacol Toxicol. 1983; 23:239-257. [PubMed: 6307121]

203. Daugherty A, Dunn JL, Rateri DL, Heinecke JW. Myeloperoxidase, a Catalyst for Lipoprotein Oxidation, is Expressed in Human Atherosclerotic Lesions. J Clin Invest. 1994; 94:437-444. [PubMed: 8040285]

204. Jones CM, Lawrence A, Wardman P, Burkitt MJ. Kinetics of Superoxide Scavenging by Glutathione: An Evaluation of its Role in the Removal of Mitochondrial Superoxide. Biochem Soc Trans. 2003; 31:1337-1339. [PubMed: 14641058]

205. Radi R. Peroxynitrite, a Stealthy Biological Oxidant. The Journal of Biological Chemistry. 2013; 288:26464-26472. [PubMed: 23861390]

206. Ferrer-Sueta G, Radi R. Chemical Biology of Peroxynitrite: Kinetics, Diffusion, and Radicals. ACS Chem Biol. 2009; 4:161-177. [PubMed: 19267456]

207. Bartesaghi S, Radi R. Fundamentals on the Biochemistry of Peroxynitrite and Protein Tyrosine Nitration. Redox Biol. 2018; 14:618-625. [PubMed: 29154193]

208. Radi R, Beckman JS, Bush KM, Freeman BA. Peroxynitrite Oxidation of Sulfhydryls. The Cytotoxic Potential of Superoxide and Nitric Oxide. J Biol Chem. 1991; 266:4244-4250. [PubMed: 1847917]

209. Alvarez B, Radi R. Peroxynitrite Reactivity with Amino Acids and Proteins. Amino Acids. 2003; 25:295-311. [PubMed: 14661092]

210. Alvarez B, Rubbo H, Kirk M, Barnes S, Freeman BA, Radi R. Peroxynitrite-Dependent Tryptophan Nitration. Chem Res Toxicol. 1996; 9:390-396. [PubMed: 8839040]

211. Augusto O, Bonini MG, Amanso AM, Linares E, Santos CCX, De Menezes SLL. Nitrogen Dioxide and Carbonate Radical Anion: Two Emerging Radicals in Biology. Free Radical Biol Med. 2002; 32:841-859. [PubMed: 11978486]

212. Augusto O, Miyamoto S. Principles of Free Radical Biomedicine. Pantopoulos K, Schipper HM, editors Vol. 1. Happauge: 2011. 
213. Bonini MG, Augusto O. Carbon Dioxide Stimulates the Production of Thiyl, Sulfinyl, and Disulfide Radical Anion from Thiol Oxidation by Peroxynitrite. J Biol Chem. 2001; 276:9749_ 9754. [PubMed: 11134018]

214. Ramirez DC, Gomez-Mejiba SE, Corbett JT, Deterding LJ, Tomer KB, Mason RP. Cu, ZnSuperoxide Dismutase-Driven Free Radical Modifications: Copper and Carbonate Radical Anion-Initiated Protein Radical Chemistry. Biochem J. 2009; 417:341-353. [PubMed: 18764780]

215. Medinas DB, Gozzo FC, Santos LFA, Iglesias AH, Augusto O. A Ditryptophan Cross-Link Is Responsible for the Covalent Dimerization of Human Superoxide Dismutase 1 during its Bicarbonate-Dependent Peroxidase Activity. Free Radical Biol Med. 2010; 49:1046-1053. [PubMed: 20600836]

216. Paviani V, Queiroz RF, Marques EF, Di Mascio P, Augusto O. Production of Lysozyme and Lysozyme-Superoxide Dismutase Dimers Bound by a Ditryptophan Cross-Link in Carbonate Radical-Treated Lysozyme. Free Radical Biol Med. 2015; 89:72-82. [PubMed: 26197052]

217. Winterbourn CC. Toxicity of Iron and Hydrogen Peroxide: The Fenton Reaction. Toxicol Lett. 1995; 82-83:969-974.

218. Kehrer JP. The Haber-Weiss Reaction and Mechanisms of Toxicity. Toxicology. 2000; 149:4350. [PubMed: 10963860]

219. Mizuta Y, Masumizu T, Kohno M, Mori A, Packer L. Kinetic Analysis of the Fenton Reaction by ESR-Spin Trapping. Biochem Mol Biol Int. 1997; 43:1107-1120. [PubMed: 9415820]

220. Lee H, Lee HJ, Sedlak DL, Lee C. pH-Dependent Reactivity of Oxidants Formed by Iron and Copper-Catalyzed Decomposition of Hydrogen Peroxide. Chemosphere. 2013; 92:652-658. [PubMed: 23433935]

221. Engelmann MD, Bobier RT, Hiatt T, Cheng IF. Variability of the Fenton Reaction Characteristics of the EDTA, DTPA, and Citrate Complexes of Iron. BioMetals. 2003; 16:519-527. [PubMed: 12779237]

222. Rachmilovich-Calis S, Masarwa A, Meyerstein N, Meyerstein D. The Effect of Pyrophosphate, Tripolyphosphate and ATP on the Rate of the Fenton Reaction. J Inorg Biochem. 2011; 105:669_ 674. [PubMed: 21450270]

223. Cabantchik ZI. Labile Iron in Cells and Body Fluids: Physiology, Pathology, and Pharmacology. Front Pharmacol. 2014; 5:45. [PubMed: 24659969]

224. Schmidt KN, Amstad P, Cerutti P, Baeuerle PA. The Roles of Hydrogen Peroxide and Superoxide as Messengers in the Activation of Transcription Factor NF- $\mathrm{kB}$. Chem Biol. 1995; 2:13-22. [PubMed: 9383399]

225. Teixeira HD, Schumacher RI, Meneghini R. Lower Intracellular Hydrogen Peroxide Levels in Cells Overexpressing Cu Zn-Superoxide Dismutase. Proc Natl Acad Sci U S A. 1998; 95:78727875. [PubMed: 9653107]

226. Steinbeck MJ, Khan AU, Karnovsky MJ. Extracellular Production of Singlet Oxygen by Stimulated Macrophages Quantified Using 9,10-Diphenylanthracene and Perylene in a Polystyrene Film. J Biol Chem. 1993; 268:15649-15654. [PubMed: 8340389]

227. Winterbourn CC, Kettle AJ, Hampton MB. Reactive Oxygen Species and Neutrophil Function. Annu Rev Biochem. 2016; 85:765-792. [PubMed: 27050287]

228. Hurst JK. What Really Happens in the Neutrophil Phagosome? Free Radical Biol Med. 2012; 53:508-520. [PubMed: 22609248]

229. Mikkelsen RB, Wardman P. Biological chemistry of reactive oxygen and nitrogen and radiationinduced signal transduction mechanisms. Oncogene. 2003; 22:5734-5754. [PubMed: 12947383]

230. Demarquoy J, Le Borgne F. Crosstalk Between Mitochondria and Peroxisomes. World J Biol Chem. 2015; 6:301-309. [PubMed: 26629313]

231. Schuldiner M, Zalckvar E. Incredibly Close-A Newly Identified Peroxisome-ER Contact Site in Humans. J Cell Biol. 2017; 216:287-289. [PubMed: 28108527]

232. Sugiura A, Mattie S, Prudent J, Mcbride HM. Newly Born Peroxisomes are a Hybrid of Mitochondrial and ER-Derived Pre-Peroxisomes. Nature. 2017; 542:251-254. [PubMed: 28146471]

233. Lismont C, Nordgren M, Van Veldhoven PP, Fransen M. Redox Interplay between Mitochondria and Peroxisomes. Front Cell Dev Biol. 2015; 3:35. [PubMed: 26075204] 
234. Cantu D, Schaack J, Patel M. Oxidative Inactivation of Mitochondrial Aconitase Results in Iron and H2O2-Mediated Neurotoxicity in Rat Primary Mesencephalic Cultures. PLoS One. 2009; 4:e7095. [PubMed: 19763183]

235. Walton PA, Pizzitelli M. Effects of Peroxisomal Catalase Inhibition on Mitochondrial Function. Front Physiol. 2012; 3:108. [PubMed: 22536190]

236. López-Erauskin J, Galino J, Ruiz M, Cuezva JM, Fabregat I, Cacabelos D, Boada J, Martínez J, Ferrer I, Pamplona R, et al. Impaired Mitochondrial Oxidative Phosphorylation in the Peroxisomal Disease X-Linked Adrenoleukodystrophy. Hum Mol Genet. 2013; 22:3296-3305. [PubMed: 23604518]

237. Gerasimenko JV, Gerasimenko OV, Palejwala A, Tepikin AV, Petersen OH, Watson AJM. Menadione-Induced Apoptosis: Roles of Cytosolic Ca2+ Elevations and the Mitochondrial Permeability Transition Pore. J Cell Sci. 2002; 115:485-497. [PubMed: 11861756]

238. Leadsham Jane eSanders G, Giannaki S, Bastow Emma lHutton R, Naeimi Wesley rBreitenbach M, Gourlay Campbell w. Loss of Cytochrome C Oxidase Promotes RAS-Dependent ROS Production from the ER Resident NADPH oxidase Yno1p in Yeast. Cell Metab. 2013; 18:279286. [PubMed: 23931758]

239. Murphy Michael p. Mitochondrial Dysfunction Indirectly Elevates ROS Production by the Endoplasmic Reticulum. Cell Metab. 2013; 18:145-146. [PubMed: 23931748]

240. Ponath V, Kaina B. Death of Monocytes Through Oxidative Burst of Macrophages and Neutrophils: Killing in Trans. PLoS One. 2017; 12:e0170347. [PubMed: 28099491]

241. Pletjushkina OY, Fetisova EK, Lyamzaev KG, Ivanova OY, Domnina LV, Vyssokikh MY, Pustovidko AV, Vasiliev JM, Murphy MP, Chernyak BV, et al. Long-Distance Apoptotic Killing of Cells is Mediated by Hydrogen Peroxide in a Mitochondrial ROS-Dependent Fashion. Cell Death Differ. 2005; 12:1442-1444. [PubMed: 15933738]

242. Niethammer P, Grabher C, Look AT, Mitchison TJ. A Tissue-Scale Gradient of Hydrogen Peroxide Mediates Rapid Wound Detection in Zebrafish. Nature. 2009; 459:996-999. [PubMed: 19494811]

243. Yoo SK, Starnes TW, Deng Q, Huttenlocher A. Lyn is a Redox Sensor that Mediates Leukocyte Wound Attraction in Vivo. Nature. 2011; 480:109-112. [PubMed: 22101434]

244. Lopachin RM, Gavin T. Reactions of Electrophiles with Nucleophilic Thiolate Sites: Relevance to Pathophysiological Mechanisms and Remediation. Free Radic Res. 2016; 50:195-205. [PubMed: 26559119]

245. Schwöbel JaHKoleva YK, Enoch SJ, Bajot F, Hewitt M, Madden JC, Roberts DW, Schultz TW, Cronin MTD. Measurement and Estimation of Electrophilic Reactivity for Predictive Toxicology. Chem Rev. 2011; 111:2562-2596. [PubMed: 21401043]

246. Jackman LM, Lange BC. Methylation of Lithioisobutyrophenone in Weakly Polar Aprotic Solvents. The Effect of Aggregation. J Am Chem Soc. 1981; 103:4494-4499.

247. Bacot S, Bernoud-Hubac N, Baddas N, Chantegrel B, Deshayes C, Doutheau A, Lagarde M, Guichardant M. Covalent Binding of Hydroxy-Alkenals 4-HDDE, 4-HHE, and 4-HNE to Ethanolamine Phospholipid Subclasses. J Lipid Res. 2003; 44:917-926. [PubMed: 12588949]

248. Guichardant M, Taibi-Tronche P, Fay LB, Lagarde M. Covalent Modifications of Aminophospholipids by 4-Hydroxynonenal. Free Radical Biol Med. 1998; 25:1049-1056. [PubMed: 9870558]

249. Cipollina C. Endogenous Generation and Signaling Actions of Omega-3 Fatty Acid Electrophilic Derivatives. BioMed Res Int. 2015; 2015:13.

250. Rouzer CA, Marnett LJ. Endocannabinoid Oxygenation by Cyclooxygenases, Lipoxygenases, and Cytochromes P450: Cross-Talk Between the Eicosanoid and Endocannabinoid Signaling Pathways. Chem Rev. 2011; 111:5899-5921. [PubMed: 21923193]

251. Long MJC, Aye Y. The Die is Cast: Precision Electrophilic Modifications Contribute to Cellular Decision Making. Chem Res Toxicol. 2016; 29:1575-1582.

252. Long MJ, Lin HY, Parvez S, Zhao Y, Poganik JR, Huang P, Aye Y. $\beta$-TrCP1 is a Vacillatory Regulator of Wnt Signaling. Cell Chem Biol. 2017; 24:944-957e947. [PubMed: 28736239]

253. Nusse R. Wnt Signaling in Disease and in Development. Cell Res. 2005; 15:28-32. [PubMed: 15686623] 
254. Gil L, Siems W, Mazurek B, Gross J, Schroeder P, Voss P, Grune T. Age-Associated Analysis of Oxidative Stress Parameters in Human Plasma and Erythrocytes. Free Radic Res. 2006; 40:495505. [PubMed: 16551576]

255. Dalleau S, Baradat M, Gueraud F, Huc L. Cell Death and Diseases Related to Oxidative Stress: 4Hydroxynonenal (HNE) in the Balance. Cell Death Differ. 2013; 20:1615-1630. [PubMed: 24096871]

256. Rossi A, Kapahi P, Natoli G, Takahashi T, Chen Y, Karin M, Santoro MG. Anti-Inflammatory Cyclopentenone Prostaglandins are Direct Inhibitors of Ixb Kinase. Nature. 2000; 403:103-118. [PubMed: 10638762]

257. Ji C, Kozak KR, Marnett LJ. IkB Kinase, a Molecular Target for Inhibition by 4-Hydroxy-2Nonenal. J Biol Chem. 2001; 276:18223-18228. [PubMed: 11359792]

258. Villacorta L, Zhang J, Garcia-Barrio MT, Chen XL, Freeman BA, Chen YE, Cui T. Nitro-linoleic Acid Inhibits Vascular Smooth Muscle Cell Proliferation via the Keap1/Nrf2 Signaling Pathway. Am J Physiol Heart Circ Physiol. 2007; 293:H770-H776. [PubMed: 17468336]

259. Parvez S, Fu Y, Li J, Long MJC, Lin HY, Lee DK, Hu GS, Aye Y. Substoichiometric Hydroxynonenylation of a Single Protein Recapitulates Whole-Cell-Stimulated Antioxidant Response. J Am Chem Soc. 2015; 137:10-13. [PubMed: 25544059]

260. Rothe T, Gruber F, Uderhardt S, Ipseiz N, Rössner S, Oskolkova O, Blüml S, Leitinger N, Bicker W, Bochkov VN, et al. 12/15-Lipoxygenase-Mediated Enzymatic Lipid Oxidation Regulates DC Maturation and Function. J Clin Invest. 2015; 125:1944-1954. [PubMed: 25844901]

261. Groeger AL, Cipollina C, Cole MP, Woodcock SR, Bonacci G, Rudolph TK, Rudolph V, Freeman BA, Schopfer FJ. Cyclooxygenase-2 generates anti-inflammatory mediators from omega-3 fatty acids. Nature Chemical Biology. 2010; 6:433. [PubMed: 20436486]

262. Cipollina C, Salvatore SR, Muldoon MF, Freeman BA, Schopfer FJ. Generation and Dietary Modulation of Anti-Inflammatory Electrophilic Omega-3 Fatty Acid Derivatives. PLoS One. 2014; 9:e94836. [PubMed: 24736647]

263. Fischer R, Konkel A, Mehling H, Blossey K, Gapelyuk A, Wessel N, Von Schacky C, Dechend R, Muller DN, Rothe M, et al. Dietary Omega-3 Fatty Acids Modulate the Eicosanoid Profile in Man Primarily via the Cyp-Epoxygenase Pathway. J Lipid Res. 2014; 55:1150-1164. [PubMed: 24634501]

264. Dennis EA, Norris PC. Eicosanoid Storm in Infection and Inflammation. Nat Rev Immunol. 2015; 15:511-523. [PubMed: 26139350]

265. Belton O, Fitzgerald DJ. Cyclooxygenase Isoforms and Atherosclerosis. Expert Rev Mol Med. 2004; 5:1-18.

266. Shibata T, Kondo M, Osawa T, Shibata N, Kobayashi M, Uchida K. 15-Deoxy- $\Delta 12,14$ prostaglandin J2: A Prostaglandin D2 Metabolite Generated During Inflammatory Processes. J Biol Chem. 2002; 277:10459-10466. [PubMed: 11786541]

267. Hoffmann C. COX-2 in Brain and Spinal Cord - Implications for Therapeutic Use. Curr Med Chem. 2000; 7:1113-1120. [PubMed: 11032961]

268. Kirkby NS, Chan MV, Zaiss AK, Garcia-Vaz E, Jiao J, Berglund LM, Verdu EF, Ahmetaj-Shala B, Wallace JL, Herschman HR, et al. Systematic Study of Constitutive Cyclooxygenase-2 Expression: Role of NF- $\mathrm{B}$ and NFAT Transcriptional Pathways. Proc Natl Acad Sci U S A. 2016; 113:434-439. [PubMed: 26712011]

269. Jones DA, Carlton DP, Mcintyre TM, Zimmerman GA, Prescott SM. Molecular Cloning of Human Prostaglandin Endoperoxide Synthase Type II and Demonstration of Expression in Response to Cytokines. J Biol Chem. 1993; 268:9049-9054. [PubMed: 8473346]

270. Chen Y, Morrow JD, Roberts LJ. Formation of Reactive Cyclopentenone Compounds in Vivo as Products of the Isoprostane Pathway. J Biol Chem. 1999; 274:10863-10868. [PubMed: 10196163]

271. Ayala A, Munoz MF, Arguelles S. Lipid Peroxidation: Production, Metabolism, and Signaling Mechanisms of Malondialdehyde and 4-Hydroxy-2-nonenal. Oxid Med Cell Longev. 2014; 2014:31.

272. Repetto MG, Ferrarotti NF, Boveris A. The Involvement of Transition Metal Ions on IronDependent Lipid Peroxidation. Arch Toxicol. 2010; 84:255-262. [PubMed: 19936709] 
273. Kappus H. Oxidative Stress. Sies H, editorAcademic Press Inc; London: 1985.

274. Tribble DL, Aw TY, Jones DP. The Pathophysiological Significance of Lipid Peroxidation in Oxidative Cell Injury. Hepatology. 1987; 7:377-387. [PubMed: 3549510]

275. Dzikovski BG, Livshits VA, Marsh D. Oxygen Permeation Profile in Lipid Membranes: Comparison with Transmembrane Polarity Profile. Biophys J. 2003; 85:1005-1012. [PubMed: 12885647]

276. Pratt DA, Mills JH, Porter NA. Theoretical Calculations of Carbon-Oxygen Bond Dissociation Enthalpies of Peroxyl Radicals Formed in the Autoxidation of Lipids. J Am Chem Soc. 2003; 125:5801-5810. [PubMed: 12733921]

277. Yin H, Xu L, Porter NA. Free Radical Lipid Peroxidation: Mechanisms and Analysis. Chem Rev. 2011; 111:5944-5972. [PubMed: 21861450]

278. Porter NA, Lehman LS, Weber BA, Smith KJ. Unified Mechanism for Polyunsaturated Fatty Acid Autoxidation. Competition of Peroxy Radical Hydrogen Atom Abstraction, $\beta$-Scission, and Cyclization. J Am Chem Soc. 1981; 103:6447-6455.

279. Schneider C, Porter NA, Brash AR. Routes to 4-Hydroxynonenal: Fundamental Issues in the Mechanisms of Lipid Peroxidation. J Biol Chem. 2008; 283:15539-15543. [PubMed: 18285327]

280. Guéraud F. 4-Hydroxynonenal Metabolites and Adducts in Pre-Carcinogenic Conditions and Cancer. Free Radical Biol Med. 2017; 111:196-208. [PubMed: 28065782]

281. Chen HJ, Gonzalez FJ, Shou M, Chung FL. 2,3-Epoxy-4-Hydroxynonanal, a Potential Lipid Peroxidation Product for Etheno Adduct Formation, is not a Substrate of Human Epoxide Hydrolase. Carcinogenesis. 1998; 19:939-943. [PubMed: 9635886]

282. Tennyson Andrew gLippard Stephen j. Generation, Translocation and Action of Nitric Oxide in Living Systems. Chem Biol. 2011; 18:1211-1220. [PubMed: 22035790]

283. Goldstein S, Lind J, Merényi G. Chemistry of Peroxynitrites as Compared to Peroxynitrates. Chem Rev. 2005; 105:2457-2470. [PubMed: 15941219]

284. Koppenol WH, Bounds PL, Nauser T, Kissner R, Ruegger H. Peroxynitrous Acid: Controversy and Consensus Surrounding an Enigmatic Oxidant. Dalton Transact. 2012; 41:13779-13787.

285. Freeman BA, Baker PRS, Schopfer FJ, Woodcock SR, Napolitano A, D'ischia M. Nitro-Fatty Acid Formation and Signaling. J Biol Chem. 2008; 283:15515-15519. [PubMed: 18285326]

286. Gorczynski MJ, Smitherman PK, Akiyama TE, Wood HB, Berger JP, King SB, Morrow CS. Activation of Peroxisome Proliferator-Activated Receptor $\gamma$ (PPAR $\gamma$ ) by Nitroalkene Fatty Acids: Importance of Nitration Position and Degree of Unsaturation. J Med Chem. 2009; 52:4631-4639. [PubMed: 19719236]

287. Cui T, Schopfer FJ, Zhang J, Chen K, Ichikawa T, Baker PRS, Batthyany C, Chacko BK, Feng X, Patel RP, et al. Nitrated Fatty Acids: Endogenous Anti-Inflammatory Signaling Mediators. J Biol Chem. 2006; 281:35686-35698. [PubMed: 16887803]

288. Akaike T, Nishida M, Fujii S. Regulation of Redox Signalling by an Electrophilic Cyclic Nucleotide. J Biochem. 2013; 153:131-138. [PubMed: 23248242]

289. Sawa T, Zaki MH, Okamoto T, Akuta T, Tokutomi Y, Kim-Mitsuyama S, Ihara H, Kobayashi A, Yamamoto M, Fujii S, et al. Protein S-Guanylation by the Biological Signal 8-Nitroguanosine 3', 5'-Cyclic Monophosphate. Nat Chem Biol. 2007; 3:727-735. [PubMed: 17906641]

290. Fujii S, Sawa T, Ihara H, Tong KI, Ida T, Okamoto T, Ahtesham AK, Ishima Y, Motohashi H, Yamamoto M, et al. The Critical Role of Nitric Oxide Signaling, via Protein S-Guanylation and Nitrated Cyclic GMP, in the Antioxidant Adaptive Response. J Biol Chem. 2010; 285:23970 23984. [PubMed: 20498371]

291. Helms Christine C, Liu X, Kim-Shapiro Daniel B. Biol Chem. 2017; 398

292. Gladwin MT. Nitrite as an Intrinsic Signaling Molecule. Nat Chem Biol. 2005; 1:245. [PubMed: 16408049]

293. Yang M, Soga T, Pollard P, Adam J. The Emerging Role of Fumarate as an Oncometabolite. Front Oncol. 2012; 2:85. [PubMed: 22866264]

294. Sullivan Lucas bMartinez-Garcia E, Nguyen H, Mullen Andrew rDufour E, Sudarshan S, Licht Jonathan dDeberardinis Ralph jChandel Navdeep s. The Proto-Oncometabolite Fumarate Binds Glutathione to Amplify ROS-Dependent Signaling. Mol Cell. 2013; 51:236-248. [PubMed: 23747014] 
295. Lin H, Su X, He B. Protein Lysine Acylation and Cysteine Succination by Intermediates of Energy Metabolism. ACS Chem Biol. 2012; 7:947-960. [PubMed: 22571489]

296. Ternette N, Yang M, Laroyia M, Kitagawa M, O'flaherty L, Wolhulter K, Igarashi K, Saito K, Kato K, Fischer R, et al. Inhibition of Mitochondrial Aconitase by Succination in Fumarate Hydratase Deficiency. Cell Rep. 2013; 3:689-700. [PubMed: 23499446]

297. Ashrafian H, Czibik G, Bellahcene M, Aksentijević D, Smith Anthony c, Mitchell Sarah j, Dodd Michael s, Kirwan J, Byrne Jonathan j, Ludwig C, et al. Fumarate is Cardioprotective via Activation of the Nrf2 Antioxidant Pathway. Cell Metab. 2012; 15:361-371. [PubMed: 22405071]

298. Mills EL, Ryan DG, Prag HA, Dikovskaya D, Menon D, Zaslona Z, Jedrychowski MP, Costa ASH, Higgins M, Hams E, et al. Itaconate is an Anti-Inflammatory Metabolite that Activates Nrf2 via Alkylation of KEAP1. Nature. 2018; 556:113-117. [PubMed: 29590092]

299. Strelko CL, Lu W, Dufort FJ, Seyfried TN, Chiles TC, Rabinowitz JD, Roberts MF. Itaconic Acid is a Mammalian Metabolite Induced During Macrophage Activation. J Am Chem Soc. 2011; 133:16386-16389. [PubMed: 21919507]

300. Meiser J, Krämer L, Sapcariu SC, Battello N, Ghelfi J, D’herouel AF, Skupin A, Hiller K. ProInflammatory Macrophages Sustain Pyruvate Oxidation through Pyruvate Dehydrogenase for the Synthesis of Itaconate and to Enable Cytokine Expression. J Biol Chem. 2016; 291:3932-3946. [PubMed: 26679997]

301. Takagi D, Inoue H, Odawara M, Shimakawa G, Miyake C. The Calvin Cycle Inevitably Produces Sugar-Derived Reactive Carbonyl Methylglyoxal During Photosynthesis: A Potential Cause of Plant Diabetes. Plant Cell Physiol. 2014; 55:333-340. [PubMed: 24406631]

302. Thornalley PJ. Pharmacology of Methylglyoxal: Formation, Modification of Proteins and Nucleic Acids, and Enzymatic Detoxification-A Role in Pathogenesis and Antiproliferative Chemotherapy. Gen Pharmacol. 1996; 27:565-573. [PubMed: 8853285]

303. Allaman I, Bélanger M, Magistretti PJ. Methylglyoxal, the Dark Side of Glycolysis. Front Neurosci. 2015; 9:23. [PubMed: 25709564]

304. Phillips SA, Thornalley PJ. The Formation of Methylglyoxal from Triose Phosphates. Eur J Biochem. 1993; 212:101-105. [PubMed: 8444148]

305. Anderson MM, Hazen SL, Hsu FF, Heinecke JW. Human Neutrophils Employ the Myeloperoxidase-Hydrogen Peroxide-Chloride System to Convert Hydroxy-Amino Acids into Glycolaldehyde, 2-Hydroxypropanal, and Acrolein. A Mechanism for the Generation of Highly Reactive Alpha-Hydroxy and Alpha,Beta-Unsaturated Aldehydes by Phagocytes at Sites of Inflammation. J Clin Invest. 1997; 99:424-432. [PubMed: 9022075]

306. Stevens JF, Maier CS. Acrolein: Sources, Metabolism, and Biomolecular Interactions Relevant to Human Health and Disease. Mol Nutr Food Res. 2008; 52:7-25. [PubMed: 18203133]

307. Moghe A, Ghare S, Lamoreau B, Mohammad M, Barve S, Mcclain C, Joshi-Barve S. Molecular Mechanisms of Acrolein Toxicity: Relevance to Human Disease. Toxicol Sci. 2015; 143:242255. [PubMed: 25628402]

308. Wick MM, Fitzgerald G. Inhibition of Reverse Transcriptase by Tyrosinase Generated Quinones Related to Levodopa and Dopamine. Chem-Biol Interact. 1981; 38:99-107. [PubMed: 6173137]

309. Ito S, Hinoshita M, Suzuki E, Ojika M, Wakamatsu K. Tyrosinase-Catalyzed Oxidation of the Leukoderma-Inducing Agent Raspberry Ketone Produces (E)-4-(3-oxo-1-butenyl)-1,2benzoquinone: Implications for Melanocyte Toxicity. Chem Res Toxicol. 2017; 30:859-868. [PubMed: 28219012]

310. Long MJC, Hedstrom L. Mushroom Tyrosinase Oxidizes Tyrosine-Rich Sequences to Allow Selective Protein Functionalization. ChemBioChem. 2012; 13:1818-1825. [PubMed: 22807021]

311. Samavat H, Kurzer MS. Estrogen Metabolism and Breast Cancer. Cancer Lett. 2015; 356:231243. [PubMed: 24784887]

312. Park SA, Lee MH, Na HK, Surh YJ. 4-Hydroxyestradiol Induces Mammary Epithelial Cell Transformation through Nrf2-Mediated Heme Oxygenase-1 Overexpression. Oncotarget. 2017; 8:164-178. [PubMed: 27438141]

313. Lu F, Zahid M, Saeed M, Cavalieri EL, Rogan EG. Estrogen Metabolism and Formation of Estrogen-DNA Adducts in Estradiol-Treated MCF-10F Cells: The Effects of 2,3,7,8- 
Tetrachlorodibenzo-p-dioxin Induction and Catechol-O-Methyltransferase Inhibition. J Steroid Biochem Mol Biol. 2007; 105:150-158. [PubMed: 17582757]

314. Ong WY, Jenner AM, Pan N, Ong CN, Halliwell B. Elevated Oxidative Stress, Iron Accumulation Around Microvessels and Increased 4-Hydroxynonenal Immunostaining in Zone 1 of the Liver Acinus in Hypercholesterolemic Rabbits. Free Radic Res. 2009; 43:241-249. [PubMed: 19184760]

315. Williams TI, Lynn BC, Markesbery WR, Lovell MA. Increased Levels of 4-Hydroxynonenal and Acrolein, Neurotoxic Markers of Lipid Peroxidation, in the Brain in Mild Cognitive Impairment and Early Alzheimer's Disease. Neurobiol Aging. 2006; 27:1094-1099. [PubMed: 15993986]

316. Sultana R, Perluigi M, Newman SF, Pierce WM, Cini C, Coccia R, Butterfield DA. Redox Proteomic Analysis of Carbonylated Brain Proteins in Mild Cognitive Impairment and Early Alzheimer's Disease. Antioxid Redox Signal. 2010; 12:327-336. [PubMed: 19686046]

317. Verrastro I, Pasha S, Tveen Jensen K, Pitt AR, Spickett CM. Mass Spectrometry-Based Methods for Identifying Oxidized Proteins in Disease: Advances and Challenges. Biomolecules. 2015; 5:378-411. [PubMed: 25874603]

318. Eggler AL, Savinov SN. Chemical and Biological Mechanisms of Phytochemical Activation of Nrf2 and Importance in Disease Prevention. Recent Adv Phytochem. 2013; 43:121-155. [PubMed: 26855455]

319. Groeger AL, Freeman BA. Signaling Actions of Electrophiles: Anti-Inflammatory Therapeutic Candidates. Mol Interventions. 2010; 10:39-50.

320. Dinkova-Kostova AT, Kostov RV. Glucosinolates and Isothiocyanates in Health and Disease. Trends Mol Med. 2012; 18:337-347. [PubMed: 22578879]

321. Zhang Y, Talalay P. Anticarcinogenic Activities of Organic Isothiocyanates: Chemistry and Mechanisms. Cancer Res. 1994; 54:1976s-1981s. [PubMed: 8137323]

322. Yuan J-M, Stepanov I, Murphy SE, Wang R, Allen S, Jensen J, Strayer L, Adams-Haduch J, Upadhyaya P, Le C, et al. Clinical Trial of 2-Phenethyl Isothiocyanate as an Inhibitor of Metabolic Activation of a Tobacco-Specific Lung Carcinogen in Cigarette Smokers. Cancer Prev Res (Phila). 2016; 9:396-405. [PubMed: 26951845]

323. Dinkova-Kostova A, Talalay P. Relation of Structure of Curcumin Analogs to Their Potencies as Inducers of Phase 2 Detoxification Enzymes. Carcinogenesis. 1999; 20:911-914. [PubMed: 10334211]

324. Huang TC, Chung YL, Wu ML, Chuang SM. Cinnamaldehyde Enhances Nrf2 Nuclear Translocation to Upregulate Phase Ii Detoxifying Enzyme Expression in HepG2 Cells. J Agric Food Chem. 2011; 59:5164-5171. [PubMed: 21469739]

325. Feng Z, Hu W, Hu Y, Tang MS. Acrolein is a Major Cigarette-Related Lung Cancer Agent: Preferential Binding at p53 Mutational Hotspots and Inhibition of DNA Repair. Proc Natl Acad Sci U S A. 2006; 103:15404-15409. [PubMed: 17030796]

326. Linnell RH, Scott WE. Diesel Exhaust Composition and Odor Studies. J Air Pollut Control Assoc. 1962; 12:510-545.

327. Jones DP, Carlson JL, Mody VC Jr, Cai J, Lynn MJ, Sternberg P Jr. Redox State of Glutathione in Human Plasma. Free Radical Biol Med. 2000; 28:625-635. [PubMed: 10719244]

328. Roede JR, Uppal K, Liang Y, Promislow DE, Wachtman LM, Jones DP. Characterization of Plasma Thiol Redox Potential in a Common Marmoset Model of Aging. Redox Biol. 2013; 1:387-393. [PubMed: 24024176]

329. Moon GJ, Shin DH, Im DS, Bang OY, Nam HS, Lee JH, Joo IS, Huh K, Gwag BJ. Identification of Oxidized Serum Albumin in the Cerebrospinal Fluid of Ischaemic Stroke Patients. Eur J Neurol. 2011; 18:1151-1158. [PubMed: 21299736]

330. Huang BK, Sikes HD. Quantifying Intracellular Hydrogen Peroxide Perturbations in Terms of Concentration. Redox Biol. 2014; 2:955-962. [PubMed: 25460730]

331. Huang BK, Sikes HD. Quantifying intracellular hydrogen peroxide perturbations in terms of concentration. Redox Biology. 2014; 2:955-962. [PubMed: 25460730]

332. Antunes F, Brito PM. Quantitative Biology of Hydrogen Peroxide Signaling. Redox Biol. 2017; 13:1-7. [PubMed: 28528123] 
333. Lawson AP, Long MJ, Coffey RT, Qian Y, Weerapana E, El Oualid F, Hedstrom L. Naturally Occurring Isothiocyanates Exert Anticancer Effects by Inhibiting Deubiquitinating Enzymes. Cancer Res. 2015; 75:5130-5142. [PubMed: 26542215]

334. Conigrave AD, Van Der Weyden L, Holt L, Jiang L, Wilson P, Christopherson RI, Morris MB. Extracellular ATP-Dependent Suppression of Proliferation and Induction of Differentiation of Human HL-60 Leukemia Cells by Distinct Mechanisms. Biochem Pharmacol. 2000; 60:15851591. [PubMed: 11077040]

335. R JwUS. Metabolism of Adenosine 5' -Monophosphate During Circulation through the Lungs. Trans Assoc Am Physicians. 1971; 84:297-306. [PubMed: 4275416]

336. Coade SB, Pearson JD. Metabolism of Adenine Nucleotides in Human Blood. Circ Res. 1989; 65:531-537. [PubMed: 2548757]

337. Huang D, Zhang Y, Chen X. Analysis of Intracellular Nucleoside Triphosphate Levels in Normal and Tumor Cell Lines by High-Performance Liquid Chromatography. J Chromatogr B: Anal Technol Biomed Life Sci. 2003; 784:101-109.

338. Bianchi V, Pontis E, Reichard P. Dynamics of the dATP Pool in Cultured Mammalian Cells. Exp Cell Res. 1992; 199:120-128. [PubMed: 1735453]

339. Wang RH, Tao L, Trumbore MW, Berger SL. Turnover of the Acyl Phosphates of Human and Murine Prothymosin a in Vivo. J Biol Chem. 1997; 272:26405-26412. [PubMed: 9334215]

340. Linder ME, Deschenes RJ. Palmitoylation: Policing Protein Stability and Traffic. Nat Rev Mol Cell Biol. 2007; 8:74-84. [PubMed: 17183362]

341. Yen HCS, Xu Q, Chou DM, Zhao Z, Elledge SJ. Global Protein Stability Profiling in Mammalian Cells. Science. 2008; 322:918-923. [PubMed: 18988847]

342. Iwami K, Wang JY, Jain R, Mccormack S, Johnson LR. Intestinal Ornithine Decarboxylase: HalfLife and Regulation by Putrescine. Am J Physiol. 1990; 258:G308-315. [PubMed: 2106270]

343. Turner MK, Abrams R, Lieberman I. Levels of Ribonucleotide Reductase Activity During the Division Cycle of the L Cell. J Biol Chem. 1968; 243:3725-3728. [PubMed: 5658547]

344. Carlson N, Rechsteiner M. Microinjection of Ubiquitin: Intracellular Distribution and Metabolism in HeLa Cells Maintained Under Normal Physiological Conditions. J Cell Biol. 1987; 104:537546. [PubMed: 3029141]

345. Lilienbaum A. Relationship Between the Proteasomal System and Autophagy. Int J Biochem Mol Biol. 2013; 4:1-26. [PubMed: 23638318]

346. Eden E, Geva-Zatorsky N, Issaeva I, Cohen A, Dekel E, Danon T, Cohen L, Mayo A, Alon U. Proteome Half-Life Dynamics in Living Human Cells. Science. 2011; 331:764-768. [PubMed: 21233346]

347. Oslund RC, Kee JM, Couvillon AD, Bhatia VN, Perlman DH, Muir TW. A Phosphohistidine Proteomics Strategy Based on Elucidation of a Unique Gas-Phase Phosphopeptide Fragmentation Mechanism. J Am Chem Soc. 2014; 136:12899-12911. [PubMed: 25156620]

348. Klumpp S, Krieglstein J. Phosphorylation and Dephosphorylation of Histidine Residues in Proteins. Eur J Biochem. 2002; 269:1067-1071. [PubMed: 11856347]

349. Lad C, Williams NH, Wolfenden R. The Rate of Hydrolysis of Phosphomonoester Dianions and the Exceptional Catalytic Proficiencies of Protein and Inositol Phosphatases. Proc Natl Acad Sci U S A. 2003; 100:5607-5610. [PubMed: 12721374]

350. Hunter T. Why Nature Chose Phosphate to Modify Proteins. Philos Trans R Soc Lond B Biol Sci. 2012; 367:2513-2516. [PubMed: 22889903]

351. Alberts B, Johnson A, Lewis J, Raff M, Roberts K, Walter P. Molecular Biology of the Cell. 4. Garland Science; New York: 2002.

352. Hu X, Srivastava SK, Xia H, Awasthi YC, Singh SV. An Alpha Class Mouse Glutathione STransferase with Exceptional Catalytic Efficiency in the Conjugation of Glutathione with 7B,8ADihydroxy-9A,10A-oxy-7,8,9,10-tetrahydrobenzo(A)pyrene. J Biol Chem. 1996; 271:3268432688. [PubMed: 8955099]

353. Bar-Even A, Noor E, Savir Y, Liebermeister W, Davidi D, Tawfik DS, Milo R. The Moderately Efficient Enzyme: Evolutionary and Physicochemical Trends Shaping Enzyme Parameters. Biochemistry. 2011; 50:4402-4410. [PubMed: 21506553] 
354. Patnaik D, Chin HG, Estève PO, Benner J, Jacobsen SE, Pradhan S. Substrate Specificity and Kinetic Mechanism of Mammalian G9a Histone H3 Methyltransferase. J Biol Chem. 2004; 279:53248-53258. [PubMed: 15485804]

355. Einstein A. Über Die Von Der Molekularkinetischen Theorie Der Wärme Geforderte Bewegung Von In Ruhenden Flüssigkeiten Suspendierten Teilchen. Ann Phys (Berlin, Ger). 1905; 322:549_ 560.

356. Labeit S, Kolmerer B, Linke WA. The Giant Protein Titin. Emerging Roles in Physiology and Pathophysiology. Circ Res. 1997; 80:290-294. [PubMed: 9012751]

357. Higdon AN, Landar A, Barnes S, Darley-Usmar VM. The Electrophile Responsive Proteome: Integrating Proteomics and Lipidomics with Cellular Function. Antioxid Redox Signal. 2012; 17:1580-1589. [PubMed: 22352679]

358. Owen JB, Butterfield DA. Measurement of Oxidized/Reduced Glutathione Ratio. Methods Mol Biol. 2010; 648:269-277. [PubMed: 20700719]

359. Milo R. What is the total number of protein molecules per cell volume? A call to rethink some published values. Bioessays. 2013; 35:1050-1055. [PubMed: 24114984]

360. Ellis RJ. Macromolecular Crowding: An Important but Neglected Aspect of the Intracellular Environment. Curr Opin Struct Biol. 2001; 11:114-119. [PubMed: 11179900]

361. Ricci G, De Maria F, Antonini G, Turella P, Bullo A, Stella L, Filomeni G, Federici G, Caccuri AM. 7-Nitro-2,1,3-benzoxadiazole Derivatives, a New Class of Suicide Inhibitors for Glutathione S-Transferases: Mechanism of Action of Potential Anticancer Drugs. J Biol Chem. 2005; 280:26397-26405. [PubMed: 15888444]

362. Kaiser SE, Riley BE, Shaler TA, Trevino RS, Becker CH, Schulman H, Kopito RR. Protein Standard Absolute Quantification (PSAQ) Method for the Measurement of Cellular Ubiquitin Pools. Nat Methods. 2011; 8:691-696. [PubMed: 21743460]

363. Hansen RE, Roth D, Winther JR. Quantifying the Global Cellular Thiol-Disulfide Status. Proc Natl Acad Sci U S A. 2009; 106:422-427. [PubMed: 19122143]

364. Cotgreave IA, Weis M, Berggren M, Sandy MS, Moldéus PW. Determination of the Intracellular Protein Thiol Distribution of Hepatocytes Using Monobromobimane Derivatisation of Intact Cells and Isolated Subcellular Fractions. J Biochem Biophys Methods. 1988; 16:247-254. [PubMed: 3221035]

365. Leeds JM, Slabaugh MB, Matthews CK. DNA Precursor Pools and Ribonucleotide Reductase Activity: Distribution Between the Nucleus and Cytoplasm of Mammalian Cells. Mol Cell Biol. 1985; 5:3443-3450. [PubMed: 3915777]

366. Go YM, Chandler JD, Jones DP. The Cysteine Proteome. Free Radical Biol Med. 2015; 84:227245. [PubMed: 25843657]

367. Marino SM, Gladyshev VN. Cysteine Function Governs its Conservation and Degeneration and Restricts its Utilization on Protein Surfaces. J Mol Biol. 2010; 404:902-916. [PubMed: 20950627]

368. Liu Q, Simpson DC, Gronert S. Carbonylation of Mitochondrial Aconitase with 4-Hydroxy-2(E)-nonenal: Localization and Relative Reactivity of Addition Sites. Biochim Biophys Acta. 2013; 1834:1144-1154. [PubMed: 23518448]

369. Ullrich O, Huser H, Ehrlich W, Grune T. Intracellular Metabolism of 4-Hydroxynonenal in Primary Cultures of Rabbit Synovial Fibroblasts. Free Radical Biol Med. 1997; 22:1153-1157. [PubMed: 9098088]

370. Valles SM, Perera OP, Strong CA. Purification, Biochemical Characterization, and cDNA Cloning of a Glutathione S-Transferase from the Red Imported Fire Ant, Solenopsis invicta. Insect Biochem Mol Biol. 2003; 33:981-988. [PubMed: 14505691]

371. Pandey T, Singh SK, Chhetri G, Tripathi T, Singh AK. Characterization of a Highly pH Stable Chi-Class Glutathione S-transferase from Synechocystis PCC 6803. PLoS One. 2015; 10:e0126811. [PubMed: 25965384]

372. Cheng JZ, Singhal SS, Saini M, Singhal J, Piper JT, Van Kuijk FJ, Zimniak P, Awasthi YC, Awasthi S. Effects of mGST A4 Transfection on 4-Hydroxynonenal-Mediated Apoptosis and Differentiation of K562 Human Erythroleukemia Cells. Arch Biochem Biophys. 1999; 372:29_ 36. [PubMed: 10562413] 
373. Ayyadevara S, Engle MR, Singh SP, Dandapat A, Lichti CF, Beneš H, Reis RJS, Liebau E, Zimniak P. Lifespan and Stress Resistance of Caenorhabditis elegans are Increased by Expression of Glutathione Transferases Capable of Metabolizing the Lipid Peroxidation Product 4Hydroxynonenal. Aging Cell. 2005; 4:257-271. [PubMed: 16164425]

374. Mcelhanon KE, Bose C, Sharma R, Wu L, Awasthi YC, Singh SP. Gsta4 Null Mouse Embryonic Fibroblasts Exhibit Enhanced Sensitivity to Oxidants: Role of 4-Hydroxynonenal in Oxidant Toxicity. Open J Apoptosis. 2013:2.

375. Singh SP, Niemczyk M, Saini D, Awasthi YC, Zimniak L, Zimniak P. Role of the Electrophilic Lipid Peroxidation Product 4-Hydroxynonenal in the Development and Maintenance of Obesity in Mice. Biochemistry. 2008; 47:3900-3911. [PubMed: 18311940]

376. Singh SP, Niemczyk M, Saini D, Sadovov V, Zimniak L, Zimniak P. Disruption of the mGsta4 Gene Increases Life Span of C57BL Mice. J Gerontol A: Biol Sci Med Sci. 2010; 65:14-23. [PubMed: 19880816]

377. Xie Z, Baba SR, Sweeney B, Barski O. Detoxification of Aldehydes by Histidine-Containing Dipeptides: From Chemistry to Clinical Implications. Chem Biol Interact. 2013; 202:288-297. [PubMed: 23313711]

378. Liu Y, Xu G, Sayre LM. Carnosine Inhibits (E)-4-Hydroxy-2-nonenal-Induced Protein CrossLinking: Structural Characterization of Carnosine-HNE Adducts. Chem Res Toxicol. 2003; 16:1589-1597. [PubMed: 14680373]

379. Mittler R, Vanderauwera S, Suzuki N, Miller G, Tognetti VB, Vandepoele K, Gollery M, Shulaev V, Van Breusegem F. ROS Signaling: the New Wave? Trends Plant Sci. 2011; 16:300-309. [PubMed: 21482172]

380. Gacesa R, Dunlap WC, Barlow DJ, Laskowski RA, Long PF. Rising Levels of Atmospheric Oxygen and Evolution of Nrf2. Sci Rep. 2016; 6:27740. [PubMed: 27297177]

381. Speijer D. Being Right on Q: Shaping Eukaryotic Evolution. Biochem J. 2016; 473:4103-4127. [PubMed: 27834740]

382. Winterbourn CC. Reconciling the chemistry and biology of reactive oxygen species. Nature chemical biology. 2008; 4:278-286. [PubMed: 18421291]

383. Wood ZA, Poole LB, Karplus PA. Peroxiredoxin Evolution and the Regulation of Hydrogen Peroxide Signaling. Science. 2003; 300:650-653. [PubMed: 12714747]

384. Hayes JD, Flanagan JU, Jowsey IR. Glutathione transferases. Annu Rev Pharmacol Toxicol. 2005; 45:51-88. [PubMed: 15822171]

385. Woo HA, Yim SH, Shin DH, Kang D, Yu DY, Rhee SG. Inactivation of Peroxiredoxin I by Phosphorylation Allows Localized H2O2 Accumulation for Cell Signaling. Cell. 2010; 140:517_ 528. [PubMed: 20178744]

386. Mishina NM, Tyurin-Kuzmin PA, Markvicheva KN, Vorotnikov AV, Tkachuk VA, Laketa V, Schultz C, Lukyanov S, Belousov VV. Does Cellular Hydrogen Peroxide Diffuse or Act Locally? Antioxid Redox Signal. 2011; 14:1-7. [PubMed: 20690882]

387. Seaver LC, Imlay JA. Hydrogen Peroxide Fluxes and Compartmentalization Inside Growing Escherichia coli. J Bacteriol. 2001; 183:7182-7189. [PubMed: 11717277]

388. Wittmann C, Chockley P, Singh SK, Pase L, Lieschke GJ, Grabher C. Hydrogen Peroxide in Inflammation: Messenger, Guide, and Assassin. Adv Hematol. 2012; 2012:541471. [PubMed: 22737171]

389. Parvez S, Long MJC, Lin HY, Zhao Y, Haegele JA, Pham VN, Lee DK, Aye Y. T-REX OnDemand Redox Targeting in Live Cells. Nat Protoc. 2016; 11:2328-2356. [PubMed: 27809314]

390. Long MJC, Aye Y. Privileged Electrophile Sensors: A Resource for Covalent Drug Development. Cell Chemical Biology. 2017; 24:787-800. [PubMed: 28648380]

391. Liebler DC. Protein Damage by Reactive Electrophiles: Targets and Consequences. Chem Res Toxicol. 2008; 21:117-128. [PubMed: 18052106]

392. Avery SV. Molecular Targets of Oxidative Stress. Biochem J. 2011; 434:201-210. [PubMed: 21309749]

393. Ji Y, Dai Z, Wu G, Wu Z. 4-Hydroxy-2-nonenal Induces Apoptosis by Activating Erk1/2 Signaling and Depleting Intracellular Glutathione in Intestinal Epithelial Cells. Sci Rep. 2016; 6:32929. [PubMed: 27620528] 
394. Lee HR, Cho JM, Shin DH, Yong CS, Choi HG, Wakabayashi N, Kwak MK. Adaptive Response to GSH Depletion and Resistance to L-Buthionine-(S,R)- Sulfoximine: Involvement of Nrf2 Activation. Mol Cell Biochem. 2008; 318:23-31. [PubMed: 18587629]

395. Hourihan JM, Moronetti Mazzeo LE, Fernandez-Cardenas LP, Blackwell TK. Cysteine Sulfenylation Directs IRE-1 to Activate the SKN-1/Nrf2 Antioxidant Response. Mol Cell. 2016; 63:553-566. [PubMed: 27540856]

396. Zhu C, Hu W, Wu H, Hu X. No Evident Dose-Response Relationship between Cellular ROS Level and its Cytotoxicity-A Paradoxical Issue in ROS-Based Cancer Therapy. Sci Rep. 2014; 4:5029. [PubMed: 24848642]

397. Lopachin RM, Barber DS. Synaptic Cysteine Sulfhydryl Groups as Targets of Electrophilic Neurotoxicants. Toxicol Sci. 2006; 94:240-255. [PubMed: 16880199]

398. Saito R, Suzuki T, Hiramoto K, Asami S, Naganuma E, Suda H, Iso T, Yamamoto H, Morita M, Baird L, et al. Characterizations of Three Major Cysteine Sensors of Keap1 in Stress Response. Mol Cell Biol. 2016; 36:271-284. [PubMed: 26527616]

399. Camarillo JM, Ullery JC, Rose KL, Marnett LJ. Electrophilic Modification Of PKM2 by 4Hydroxynonenal And 4-Oxononenal Results in Protein Cross-Linking and Kinase Inhibition. Chem Res Toxicol. 2017; 30:635-641. [PubMed: 27978618]

400. Fournier RL. Basic Transport Phenomena in Biomedical Engineering. 3. Taylor \& Francis; Philadelphia: 1998.

401. Srivastava A, Krishnamoorthy G. Cell Type and Spatial Location Dependence of Cytoplasmic Viscosity Measured by Time-Resolved Fluorescence Microscopy. Arch Biochem Biophys. 1997; 340:159-167. [PubMed: 9143317]

402. Baum M, Erdel F, Wachsmuth M, Rippe K. Retrieving the Intracellular Topology from MultiScale Protein Mobility Mapping in Living Cells. Nat Commun. 2014; 5:4494. [PubMed: 25058002]

403. Wlodawer A, Minor W, Dauter Z, Jaskolski M. Protein Crystallography for NonCrystallographers, or How to Get the Best (But Not More) from Published Macromolecular Structures. FEBS J. 2008; 275:1-21.

404. Weibel ER, Staubli W, Gnagi HR, Hess FA. Correlated Morphometric and Biochemical Studies on the Liver Cell. I. Morphometric Model, Stereologic Methods, and Normal Morphometric Data for Rat Liver. J Cell Biol. 1969; 42:68-91. [PubMed: 4891915]

405. Webb BA, Chimenti M, Jacobson MP, Barber DL. Dysregulated pH: a Perfect Storm for Cancer Progression. Nat Rev Cancer. 2011; 11:671-677. [PubMed: 21833026]

406. Romantsov T, Battle AR, Hendel JL, Martinac B, Wood JM. Protein Localization in Escherichia coli Cells: Comparison of the Cytoplasmic Membrane Proteins ProP, LacY, ProW, AqpZ, MscS, and MscL. J Bacteriol. 2010; 192:912-924. [PubMed: 20008071]

407. Giese M, Albrecht M, Rissanen K. Experimental Investigation of Anion-[Small Pi] Interactions Applications and Biochemical Relevance. Chem Commun. 2016; 52:1778-1795.

408. Lloyd JB. Disulphide Reduction in Lysosomes. The Role of Cysteine. Biochem J. 1986; 237:271272. [PubMed: 3800880]

409. Trujillo M, Clippe A, Manta B, Ferrer-Sueta G, Smeets A, Declercq JP, Knoops B, Radi R. PreSteady State Kinetic Characterization of Human Peroxiredoxin 5: Taking Advantage of Trp84 Fluorescence Increase Upon Oxidation. Arch Biochem Biophys. 2007; 467:95-106. [PubMed: 17892856]

410. Kokesh FC, Westheimer FH. Reporter Group at the Active Site of Acetoacetate Decarboxylase. II. Ionization Constant of the Amino Group. J Am Chem Soc. 1971; 93:7270-7274. [PubMed: 5127416]

411. Quinn DM, Sikorski RS. Encyclopedia of Life Sciences. John Wiley \& Sons, Ltd; 2001.

412. Morrow CS, Smitherman PK, Diah SK, Schneider E, Townsend AJ. Coordinated Action of Glutathione S-Transferases (GSTs) and Multidrug Resistance Protein 1 (MRP1) in Antineoplastic Drug Detoxification: Mechanism of Gst A1-1- And Mrp1-Associated Resistance To Chlorambucil In Mcf7 Breast Carcinoma Cells. J Biol Chem. 1998; 273:20114-20120. [PubMed: 9685354] 
413. Lengfelder E, Cadenas E, Sies H. Effect of DABCO (1,4-diazabicyclo[2,2,2]-octane) on Singlet Oxygen Monomol (1270 nm) and Dimol (634 and $703 \mathrm{~nm}$ ) Emission. FEBS Lett. 1983; 164:366-370.

414. Kholodenko AL, Douglas JF. Generalized Stokes-Einstein Equation for Spherical Particle Suspensions. Phys Rev E: Stat Phys, Plasmas, Fluids, Relat Interdiscip Top. 1995; 51:1081-1090.

415. Spasojević I, Bogdanović Pristov J, Vujisić L, Spasić M. The Reaction of Methionine with Hydroxyl Radical: Reactive Intermediates and Methanethiol Production. Amino Acids. 2012; 42:2439-2445. [PubMed: 21830117]

416. Valentine WN, Toohey JI, Paglia DE, Nakatani M, Brockway RA. Modification of Erythrocyte Enzyme Activities by Persulfides and Methanethiol: Possible Regulatory Role. Proc Natl Acad Sci U S A. 1987; 84:1394-1398. [PubMed: 3469673]

417. Buettner GR. The Pecking Order of Free Radicals and Antioxidants: Lipid Peroxidation, AlphaTocopherol, and Ascorbate. Arch Biochem Biophys. 1993; 300:535-543. [PubMed: 8434935]

418. Abreu IA, Cabelli DE. Superoxide Dismutases-A Review of the Metal-Associated Mechanistic Variations. Biochim Biophys Acta. 2010; 1804:263-274. [PubMed: 19914406]

419. Hsu JL, Hsieh Y, Tu C, O'connor D, Nick HS, Silverman DN. Catalytic Properties of Human Manganese Superoxide Dismutase. J Biol Chem. 1996; 271:17687-17691. [PubMed: 8663465]

420. Zhang N, Schuchmann HP, Von Sonntag C. The Reaction of Superoxide Radical Anion with Dithiothreitol: A Chain Process. J Phys Chem A. 1991; 95:4718-4722.

421. Dikalov S, Khramtsov V, Zimmer G. Determination of Rate Constants of the Reactions of Thiols with Superoxide Radical by Electron Paramagnetic Resonance: Critical Remarks on Spectrophotometric Approaches. Arch Biochem Biophys. 1996; 326:207-218. [PubMed: 8611025]

422. Winterbourn C, Metodiewa D. The Reaction of Superoxide with Reduced Glutathione. Arch Biochem Biophys. 1994; 314:284-290. [PubMed: 7979367]

423. Antunes F, Cadenas E. Estimation of $\mathrm{H} 2 \mathrm{O} 2$ gradients across biomembranes. FEBS Lett. 2000; 475:121-126. [PubMed: 10858501]

424. Ogilby PR. Singlet Oxygen: There is Indeed Something New Under the Sun. Chem Soc Rev. 2010; 39:3181-3209. [PubMed: 20571680]

425. Skovsen E, Snyder JW, Lambert JDC, Ogilby PR. Lifetime and Diffusion of Singlet Oxygen in a Cell. J Phys Chem B. 2005; 109:8570-8573. [PubMed: 16852012]

426. Moan J, Berg K. The Photodegradation of Porphyrins in Cells Can Be Used to Estimate the Lifetime of Singlet Oxygen. Photochem Photobiol. 1991; 53:549-553. [PubMed: 1830395]

427. Dysart JS, Patterson MS. Characterization of Photofrin Photobleaching for Singlet Oxygen Dose Estimation During Photodynamic Therapy of MLL Cells in Vitro. Phys Med Biol. 2005; 50:2597-2616. [PubMed: 15901957]

428. Souslova EA, Mironova KE, Deyev SM. Applications of Genetically Encoded Photosensitizer MiniSOG: From Correlative Light Electron Microscopy to Immunophotosensitizing. J Biophotonics. 2017; 10:338-352. [PubMed: 27435584]

429. Berneburg M, Plettenberg H, Medve-Konig K, Pfahlberg A, Gers-Barlag H, Gefeller O, Krutmann J. Induction of the Photoaging-Associated Mitochondrial Common Deletion in Vivo in Normal Human Skin. J Invest Dermatol. 2004; 122:1277-1283. [PubMed: 15140232]

430. Baier J, Maisch T, Maier M, Landthaler M, Baumler W. Direct Detection of Singlet Oxygen Generated by UVA Irradiation in Human Cells and Skin. J Invest Dermatol. 2007; 127:14981506. [PubMed: 17363921]

431. Winterbourn CC, Metodiewa D. Reactivity of Biologically Important Thiol Compounds with Superoxide and Hydrogen Peroxide. Free Radical Biol Med. 1999; 27:322-328. [PubMed: 10468205]

432. Luo D, Smith SW, Anderson BD. Kinetics and Mechanism of the Reaction of Cysteine and Hydrogen Peroxide in Aqueous Solution. J Pharm Sci. 2005; 94:304-316. [PubMed: 15570599]

433. Kohler H, Jenzer H. Interaction of Lactoperoxidase with Hydrogen Peroxide: Formation of Enzyme Intermediates and Generation of Free Radicals. Free Radical Biol Med. 1989; 6:323339. [PubMed: 2545551] 
434. Heckert EG, Seal S, Self WT. Fenton-Like Reaction Catalyzed by the Rare Earth Inner Transition Metal Cerium. Environ Sci Technol. 2008; 42:5014-5019. [PubMed: 18678042]

435. Ma Y, Liu Z, Hider RC, Petrat F. Determination of the Labile Iron Pool of Human Lymphocytes Using the Fluorescent Probe, CP655. Anal Chem Insights. 2007; 2:61-67. [PubMed: 19662178]

436. Epsztejn S, Kakhlon O, Glickstein H, Breuer W, Cabantchik I. Fluorescence Analysis of the Labile Iron Pool of Mammalian Cells. Anal Biochem. 1997; 248:31-40. [PubMed: 9177722]

437. Lindahl PA, Moore MJ. Labile Low-Molecular-Mass Metal Complexes in Mitochondria: Trials and Tribulations of a Burgeoning Field. Biochemistry. 2016; 55:4140-4153. [PubMed: 27433847]

438. Jung YS, Lim WT, Park JY, Kim YH. Effect of pH on Fenton and Fenton-Like Oxidation. Environ Technol. 2009; 30:183-190. [PubMed: 19278159]

439. Sibille JC, Doi K, Aisen P. Hydroxyl Radical Formation and Iron-Binding Proteins. Stimulation by the Purple Acid Phosphatases. J Biol Chem. 1987; 262:59-62. [PubMed: 3025217]

440. Mitić N, Smith SJ, Neves A, Guddat LW, Gahan LR, Schenk G. The Catalytic Mechanisms of Binuclear Metallohydrolases. Chem Rev. 2006; 106:3338-3363. [PubMed: 16895331]

441. Luo W, Li H, Zhang Y, Ang CY. Determination of Formaldehyde in Blood Plasma by HighPerformance Liquid Chromatography with Fluorescence Detection. J Chromatogr B: Biomed Sci Appl. 2001; 753:253-257. [PubMed: 11334338]

442. Burgos-Barragan G, Wit N, Meiser J, Dingler FA, Pietzke M, Mulderrig L, Pontel LB, Rosado IV, Brewer TF, Cordell RL, et al. Mammals Divert Endogenous Genotoxic Formaldehyde into OneCarbon Metabolism. Nature. 2017; 548:549. [PubMed: 28813411]

443. Chaplen FWR, Fahl WE, Cameron DC. Evidence of High Levels of Methylglyoxal in Cultured Chinese Hamster Ovary Cells. Proc Natl Acad Sci U S A. 1998; 95:5533-5538. [PubMed: 9576917]

444. Lee DY, Chang GD. Methylglyoxal in Cells Elicits a Negative Feedback Loop Entailing Transglutaminase 2 and Glyoxalase 1. Redox Biol. 2014; 2:196-205. [PubMed: 24494193]

445. Esterbauer H, Zollner H, Scholz N. Reaction of Glutathione with Conjugated Carbonyls. Z Naturforsch C: Biochem, Biophys, Biol Virol. 1975; 30:466-473.

446. Fritz KS, Petersen DR. An Overview of the Chemistry and Biology of Reactive Aldehydes. Free Radical Biol Med. 2013; 59:85-91. [PubMed: 22750507]

447. Witz G. Biological Interactions of a, $\beta$-Unsaturated Aldehydes. Free Radical Biol Med. 1989; 7:333-349. [PubMed: 2673948]

448. Ahn NT, Eisenstein O. Theoretical Interpretation of 1-2 Asymmetric Induction. The Importance of Antiperiplanarity. Nouv J Chim. 1977; 1:61-70.

449. Gineityte V. On the Origin of the Enhanced Reactivity of a-Halocarbonyl Compounds in SN2 Processes. J Mol Struct: THEOCHEM. 2003; 663:47-58.

450. Doorn JA, Petersen DR. Covalent Adduction of Nucleophilic Amino Acids by 4-Hydroxynonenal And 4-Oxononenal. Chem Biol Interact. 2003; 143-144:93-100.

451. Esterbauer H, Gebicki J, Puhl H, Jurgens G. The Role of Lipid Peroxidation and Antioxidants in Oxidative Modification of LDL. Free Radical Biol Med. 1992; 13:341-390. [PubMed: 1398217]

452. Ishikawa T, Esterbauer H, Sies H. Role of Cardiac Glutathione Transferase and of the Glutathione S-Conjugate Export System in Biotransformation of 4-Hydroxynonenal in the Heart. J Biol Chem. 1986; 261:1576-1581. [PubMed: 3753704]

453. Wang, Kang, Wu, Tang, Pan, Ye, Tang, Li, Gao. Changes in Cardiac Mitochondrial Aldehyde Dehydrogenase 2 Activity in Relation to Oxidative Stress and Inflammatory Injury in Diabetic Rats. Mol Med Report. 2013; 8:686-690.

454. Awasthi S, Singhal SS, Sharma R, Zimniak P, Awasthi YC. Transport of Glutathione Conjugates and Chemotherapeutic Drugs by RLIP76 (RALBP1): A Novel Link Between G-Protein and Tyrosine Kinase Signaling and Drug Resistance. Int J Cancer. 2003; 106:635-646. [PubMed: 12866021]

455. Hartley DP, Ruth JA, Petersen DR. The Hepatocellular Metabolism of 4-Hydroxynonenal by Alcohol Dehydrogenase, Aldehyde Dehydrogenase, and Glutathione S-Transferase'. Arch Biochem Biophys. 1995; 316:197-205. [PubMed: 7840616] 
456. Rudolph V, Schopfer FJ, Khoo NKH, Rudolph TK, Cole MP, Woodcock SR, Bonacci G, Groeger AL, Golin-Bisello F, Chen C-S, et al. Nitro-Fatty Acid Metabolome: Saturation, Desaturation, BOxidation, and Protein Adduction. J Biol Chem. 2009; 284:1461-1473. [PubMed: 19015269]

457. Salvador A, Sousa J, Pinto RE. Hydroperoxyl, Superoxide and $\mathrm{pH}$ Gradients in the Mitochondrial Matrix: A Theoretical Assessment. Free Radical Biol Med. 2001; 31:1208-1215. [PubMed: 11705699]

458. Paoli M, Marles-Wright J, Smith A. Structure-Function Relationships in Heme-Proteins. DNA Cell Biol. 2002; 21:271-280. [PubMed: 12042067]

459. Lynch RE, Fridovich I. Effects of Superoxide on the Erythrocyte Membrane. J Biol Chem. 1978; 253:1838-1845. [PubMed: 204632]

460. Hawkins BJ, Madesh M, Kirkpatrick CJ, Fisher AB. Superoxide flux in endothelial cells via the chloride channel-3 mediates intracellular signaling. Mol Biol Cell. 2007; 18:2002-2012. [PubMed: 17360969]

461. Zuo L, Pasniciuc S, Wright VP, Merola AJ, Clanton TL. Sources for Superoxide Release: Lessons from Blockade of Electron Transport, NADPH Oxidase, and Anion Channels in Diaphraghm. Antioxid Redox Signal. 2003; 5:667-675. [PubMed: 14580324]

462. Stone JR, Yang S. Hydrogen Peroxide: A Signaling Messenger. Antioxid Redox Signal. 2006; 8:243-270. [PubMed: 16677071]

463. Bienert GP, Moller AL, Kristiansen KA, Schulz A, Moller IM, Schjoerring JK, Jahn TP. Specific Aquaporins Facilitate the Diffusion of Hydrogen Peroxide Across Membranes. J Biol Chem. 2007; 282:1183-1192. [PubMed: 17105724]

464. Sokolov VS, Pohl P. Membrane Transport of Singlet Oxygen Monitored by Dipole Potential Measurements. Biophys J. 2009; 96:77-85. [PubMed: 18931253]

465. Kushmerick MJ, Podolsky RJ. Ionic Mobility in Muscle Cells. Science. 1969; 166:1297-1298. [PubMed: 5350329]

466. Hubley MJ, Rosanske RC, Moerland TS. Diffusion Coefficients of ATP and Creatine Phosphate in Isolated Muscle: Pulsed Gradient 31P NMR of Small Biological Samples. NMR Biomed. 1995; 8:72-78. [PubMed: 7547189]

467. De Graaf RA, Van Kranenburg A, Nicolay K. In Vivo 31P-NMR Diffusion Spectroscopy of ATP and Phosphocreatine in Rat Skeletal Muscle. Biophys J. 2000; 78:1657-1664. [PubMed: 10733948]

468. Arrio-Dupont M, Foucault G, Vacher M, Devaux PF, Cribier S. Translational Diffusion of Globular Proteins in the Cytoplasm of Cultured Muscle Cells. Biophys J. 2000; 78:901-907. [PubMed: 10653802]

469. Di Rienzo C, Piazza V, Gratton E, Beltram F, Cardarelli F. Probing Short-Range Protein Brownian Motion in the Cytoplasm of Living Cells. Nat Commun. 2014; 5:5891. [PubMed: 25532887]

470. Seksek O, Biwersi J, Verkman AS. Translational Diffusion of Macromolecule-Sized Solutes in Cytoplasm and Nucleus. J Cell Biol. 1997; 138:131-142. [PubMed: 9214387]

471. Verkman AS. Solute and Macromolecule Diffusion in Cellular Aqueous Compartments. Trends Biochem Sci. 2002; 27:27-33. [PubMed: 11796221]

472. Wang R, Brattain MG. The Maximal Size of Protein to Diffuse Through the Nuclear Pore is Larger than $60 \mathrm{kDa}$. FEBS Lett. 2007; 581:3164-3170. [PubMed: 17588566]

473. Skovgaard M, Jensen LJ, Brunak S, Ussery D, Krogh A. On the Total Number of Genes and Their Length Distribution in Complete Microbial Genomes. Trends Genet. 2001; 17:425-428. [PubMed: 11485798]

474. Milo R, Phillips R. Cell Biology by the Numbers. Garland Science; New York: 2015.

475. Ferguson GP, Booth IR. Importance of Glutathione for Growth and Survival of Escherichia coli Cells: Detoxification of Methylglyoxal and Maintenance of Intracellular K+ J Bacteriol. 1998; 180:4314-4318. [PubMed: 9696786]

476. Hummel CS, Lancaster KM, Crane EJ. Determination of Coenzyme A Levels in Pyrococcus Furiosus and Other Archaea: Implications for a General Role for Coenzyme A in Thermophiles. FEMS Microbiol Lett. 2005; 252:229-234. [PubMed: 16213671] 
477. Antelmann H, Hamilton CJ. Bacterial Mechanisms of Reversible Protein S-Thiolation: Structural and Mechanistic Insights into Mycoredoxins. Mol Microbiol. 2012; 86:759-764. [PubMed: 22998128]

478. Perera VR, Newton GL, Pogliano K. Bacillithiol: a Key Protective Thiol in Staphylococcus aureus. Expert Rev Anti Infect Ther. 2015; 13:1089-1107. [PubMed: 26184907]

479. Jung T, Engels M, Kaiser B, Grune T. Distribution of Oxidized and HNE-Modified Proteins in U87 Cells. BioFactors. 2005; 24:165-170. [PubMed: 16403977]

480. Warren EaKNetterfield TS, Sarkar S, Kemp ML, Payne CK. Spatially-Resolved Intracellular Sensing of Hydrogen Peroxide in Living Cells. Sci Rep. 2015; 5:16929. [PubMed: 26585385]

481. Malinouski M, Zhou Y, Belousov VV, Hatfield DL, Gladyshev VN. Hydrogen Peroxide Probes Directed to Different Cellular Compartments. PLoS One. 2011; 6:e14564. [PubMed: 21283738]

482. Lo Conte M, Carroll KS. The Redox Biochemistry of Protein Sulfenylation and Sulfinylation. J Biol Chem. 2013; 288:26480-26488. [PubMed: 23861405]

483. Shaked ZE, Szajewski RP, Whitesides GM. Rates of Thiol-Disulfide Interchange Reactions Involving Proteins and Kinetic Measurements of Thiol pKa Values. Biochemistry. 1980; 19:4156-4166. [PubMed: 6251863]

484. Paulsen CE, Truong TH, Garcia FJ, Homann A, Gupta V, Leonard SE, Carroll KS. PeroxideDependent Sulfenylation of the EGFR Catalytic Site Enhances Kinase Activity. Nat Chem Biol. 2011; 8:57-64. [PubMed: 22158416]

485. Chang TS, Jeong W, Woo HA, Lee SM, Park S, Rhee SG. Characterization of Mammalian Sulfiredoxin and its Reactivation of Hyperoxidized Peroxiredoxin through Reduction of Cysteine Sulfinic Acid in the Active Site to Cysteine. J Biol Chem. 2004; 279:50994-51001. [PubMed: 15448164]

486. Wood ZA, Poole LB, Karplus PA. Peroxiredoxin Evolution and the Regulation of Hydrogen Peroxide Signaling. Science. 2003; 300:650-653. [PubMed: 12714747]

487. Sunico CR, Sultan A, Nakamura T, Dolatabadi N, Parker J, Shan B, Han X, Yates JR, Masliah E, Ambasudhan R, et al. Role of Sulfiredoxin as a Peroxiredoxin-2 Denitrosylase in Human IPSCDerived Dopaminergic Neurons. Proc Natl Acad Sci U S A. 2016; 113:E7564-E7571. [PubMed: 27821734]

488. Kwon YT, Kashina AS, Davydov IV, Hu RG, An JY, Seo JW, Du F, Varshavsky A. An Essential Role of N-Terminal Arginylation in Cardiovascular Development. Science. 2002; 297:96-99. [PubMed: 12098698]

489. Sriram SM, Kim BY, Kwon YT. The N-end Rule Pathway: Emerging Functions and Molecular Principles of Substrate Recognition. Nat Rev Mol Cell Biol. 2011; 12:735-747. [PubMed: 22016057]

490. Buxton GV, Greenstock CL, Helman WP, Ross AB. Critical Review of Rate Constants for Reactions of Hydrated Electrons, Hydrogen Atoms and Hydroxyl Radicals (.OH/.O-) in Aqueous Solution. J Phys Chem Ref Data. 1988; 17:513-886.

491. Hawkins CL, Davies MJ. Generation and propagation of radical reactions on proteins. Biochim Biophys Acta. 2001; 1504:196-219. [PubMed: 11245785]

492. De Vleeschouwer F, Van Speybroeck V, Waroquier M, Geerlings P, De Proft F. Electrophilicity and Nucleophilicity Index for Radicals. Org Lett. 2007; 9:2721-2724. [PubMed: 17559221]

493. Requena JR, Chao CC, Levine RL, Stadtman ER. Glutamic and Aminoadipic Semialdehydes are the Main Carbonyl Products of Metal-Catalyzed Oxidation of Proteins. Proc Natl Acad Sci U S A. 2001; 98:69-74. [PubMed: 11120890]

494. Møller IM, Rogowska-Wrzesinska A, Rao RSP. Protein Carbonylation and Metal-Catalyzed Protein Oxidation in a Cellular Perspective. J Proteomics. 2011; 74:2228-2242. [PubMed: 21601020]

495. Weng SL, Huang KY, Kaunang FJ, Huang CH, Kao HJ, Chang TH, Wang HY, Lu JJ, Lee TY. Investigation and Identification of Protein Carbonylation Sites Based on Position-Specific Amino Acid Composition and Physicochemical Features. BMC Bioinformatics. 2017; 18:66. [PubMed: 28361707]

496. Viehe HG, Janousek Z, Merenyi R, Stella L. The Captodative Effect. Acc Chem Res. 1985; 18:148-154. 
497. Stadtman ER. Oxidation of Free Amino Acids and Amino Acid Residues in Proteins by Radiolysis and by Metal-Catalyzed Reactions. Annu Rev Biochem. 1993; 62:797-821. [PubMed: 8352601]

498. Takamoto K, Chance MR. Radiolytic Protein Footprinting with Mass Spectrometry to Probe the Structure of Macromolecular Complexes. Annu Rev Biophys Biomol Struct. 2006; 35:251-276. [PubMed: 16689636]

499. Watts ZI, Easton CJ. Peculiar Stability of Amino Acids and Peptides from a Radical Perspective. J Am Chem Soc. 2009; 131:11323-11325. [PubMed: 19630427]

500. Scheiner S, Kar T. Analysis of the Reactivities of Protein C-H Bonds to H Atom Abstraction by OH Radical. J Am Chem Soc. 2010; 132:16450-16459. [PubMed: 21047072]

501. Schauenstein E, Taufer M, Esterbauer H, Kylianek A, Seelich T. Über die Reaktion von ProteinSH-Gruppen mit 4-Hydroxypentenal. Monatshefte für Chemie /Chemical Monthly. 1971; 102:517-529.

502. Siems WG, Hapner SJ, Van Kuijk FJGM. 4-Hydroxynonenal Inhibits Na+-K+-ATPase. Free Radical Biol Med. 1996; 20:215-223. [PubMed: 8746442]

503. Just J, Jung T, Friis NA, Lykkemark S, Drasbek K, Siboska G, Grune T, Kristensen P. Identification of an Unstable 4-Hydroxynoneal Modification on the 20S Proteasome Subunit a 7 by Recombinant Antibody Technology. Free Radical Biol Med. 2015; 89:786-792. [PubMed: 26472192]

504. Batthyany C, Schopfer FJ, Baker PRS, Durán R, Baker LMS, Huang Y, Cerveñansky C, Branchaud BP, Freeman BA. Reversible Post-Translational Modification of Proteins by Nitrated Fatty Acids in Vivo. J Biol Chem. 2006; 281:20450-20463. [PubMed: 16682416]

505. Rudolph TK, Freeman BA. Transduction of Redox Signaling by Electrophile-Protein Reactions. Sci Signaling. 2009; 2:re7-re7.

506. Zhang XM, Bordwell FG, Van Der Puy M, Fried HE. Equilibrium Acidities and Homolytic Bond Dissociation Energies of the Acidic Carbon-Hydrogen Bonds in N-Substituted Trimethylammonium and Pyridinium Cations. J Org Chem. 1993; 58:3060-3066.

507. Matthews WS, Bares JE, Bartmess JE, Bordwell FG, Cornforth FJ, Drucker GE, Margolin Z, Mccallum RJ, Mccollum GJ, Vanier NR. Equilibrium Acidities of Carbon Acids. Vi. Establishment of an Absolute Scale of Acidities in Dimethyl Sulfoxide Solution. J Am Chem Soc. 1975; 97:7006-7014.

508. Serafimova IM, Pufall MA, Krishnan S, Duda K, Cohen MS, Maglathlin RL, Mcfarland JM, Miller RM, Frödin M, Taunton J. Reversible Targeting of Noncatalytic Cysteines with Chemically Tuned Electrophiles. Nat Chem Biol. 2012; 8:471-476. [PubMed: 22466421]

509. Poganik JR, Long MJC, Aye Y. Getting the Message? Native Reactive Electrophiles Pass Two Out of Three Thresholds to Be Bona Fide Signaling Mediators. Bioessays. 2018; 40:e1700240. [PubMed: 29603288]

510. Globisch M, Kaden D, Henle T. 4-Hydroxy-2-nonenal (4-HNE) and its Lipation Product 2Pentylpyrrole Lysine (2-PPL) in Peanuts. J Agric Food Chem. 2015; 63:5273-5281. [PubMed: 25945920]

511. Mcmahon M, Lamont DJ, Beattie KA, Hayes JD. Keap1 Perceives Stress via Three Sensors for the Endogenous Signaling Molecules Nitric Oxide, Zinc, and Alkenals. Proc Natl Acad Sci U S A. 2010; 107:18838-18843. [PubMed: 20956331]

512. Long MJC, Parvez S, Zhao Y, Surya SL, Wang Y, Zhang S, Aye Y. Akt3 is a Privileged First Responder in Isozyme-Specific Electrophile Response. Nat Chem Biol. 2017; 13:333-338. [PubMed: 28114274]

513. Uchida K, Stadtman ER. Modification of Histidine Residues in Proteins by Reaction with 4Hydroxynonenal. Proc Natl Acad Sci U S A. 1992; 89:4544-4548. [PubMed: 1584790]

514. Ushio-Fukai M. Compartmentalization of Redox Signaling through NADPH Oxidase-Derived ROS. Antioxid Redox Signal. 2009; 11:1289-1299. [PubMed: 18999986]

515. Heppner DE, Janssen-Heininger YMW, Van Der Vliet A. The Role of Sulfenic Acids in Cellular Redox Signaling: Reconciling Chemical Kinetics and Molecular Detection Strategies. Arch Biochem Biophys. 2017; 616:40-46. [PubMed: 28126370] 
516. Kim G, Weiss SJ, Levine RL. Methionine Oxidation and Reduction in Proteins. Biochim Biophys Acta. 2014; 1840:901-905. [PubMed: 23648414]

517. Trindade DF, Cerchiaro G, Augusto O. A Role for Peroxymonocarbonate in the Stimulation of Biothiol Peroxidation by the Bicarbonate/Carbon Dioxide Pair. Chem Res Toxicol. 2006; 19:1475-1482. [PubMed: 17112235]

518. Richardson DE, Regino CaS, Yao H, Johnson JV. Methionine Oxidation by Peroxymonocarbonate, a Reactive Oxygen Species Formed from CO2/Bicarbonate and Hydrogen Peroxide. Free Radical Biol Med. 2003; 35:1538-1550. [PubMed: 14680677]

519. Zhou H, Singh H, Parsons ZD, Lewis SM, Bhattacharya S, Seiner DR, Labutti JN, Reilly TJ, Tanner JJ, Gates KS. The Biological Buffer Bicarbonate/CO2 Potentiates H2O2-Mediated Inactivation of Protein Tyrosine Phosphatases. J Am Chem Soc. 2011; 133:15803-15805. [PubMed: 21913686]

520. Homer NZ, Reglinski J, Sowden R, Spickett CM, Wilson R, Walker JJ. Dimethylsulfoxide Oxidizes Glutathione in Vitro and in Human Erythrocytes: Kinetic Analysis by 1H NMR. Cryobiology. 2005; 50:317-324. [PubMed: 15925582]

521. Trinquier G. Double Bonds and Bridged Structures in the Heavier Analogues of Ethylene. J Am Chem Soc. 1990; 112:2130-2137.

522. Moskovitz J. Methionine Sulfoxide Reductases: Ubiquitous Enzymes Involved in Antioxidant Defense, Protein Regulation, and Prevention of Aging-Associated Diseases. Biochim Biophys Acta. 2005; 1703:213-219. [PubMed: 15680229]

523. Kaya A, Lee BC, Gladyshev VN. Regulation of Protein Function by Reversible Methionine Oxidation and the Role of Selenoprotein MsrB1. Antioxid Redox Signal. 2015; 23:814-822. [PubMed: 26181576]

524. Shao B. Site-Specific Oxidation of Apolipoprotein A-I Impairs Cholesterol Export by Abca1, a Key Cardioprotective Function of HDL. Biochim Biophys Acta. 2012; 1821:490-501. [PubMed: 22178192]

525. Manta B, Gladyshev VN. Regulated Methionine Oxidation by Monooxygenases. Free Radical Biol Med. 2017; 109:141-155. [PubMed: 28229915]

526. Traoré DaKGhazouani AE, Jacquamet L, Borel F, Ferrer J-L, Lascoux D, Ravanat J-L, Jaquinod M, Blondin G, Caux-Thang C. , et al. Structural and Functional Characterization of 2-OxoHistidine in Oxidized PerR Protein. Nat Chem Biol. 2008; 5:53. [PubMed: 19079268]

527. Yang M, Chowdhury R, Ge W, Hamed RB, Mcdonough MA, Claridge TD, Kessler BM, Cockman ME, Ratcliffe PJ, Schofield CJ. Factor-Inhibiting Hypoxia-Inducible Factor (FIH) Catalyses the Post-Translational Hydroxylation of Histidinyl Residues within Ankyrin Repeat Domains. FEBS J. 2011; 278:1086-1097. [PubMed: 21251231]

528. Szweda LI, Uchida K, Tsai L, Stadtman ER. Inactivation of Glucose-6-Phosphate Dehydrogenase by 4-Hydroxy-2-nonenal. Selective Modification of an Active-Site Lysine. J Biol Chem. 1993; 268:3342-3347. [PubMed: 8429010]

529. Colzani M, Criscuolo A, De Maddis D, Garzon D, Yeum KJ, Vistoli G, Carini M, Aldini G. A Novel High Resolution MS Approach for the Screening of 4-Hydroxy-Trans-2-Nonenal Sequestering Agents. J Pharm Biomed Anal. 2014; 91:108-118. [PubMed: 24463041]

530. Uchida K, Stadtman ER. Covalent Attachment of 4-Hydroxynonenal to Glyceraldehyde-3Phosphate Dehydrogenase. A Possible Involvement of Intra- and Intermolecular Cross-Linking Reaction. J Biol Chem. 1993; 268:6388-6393. [PubMed: 8454610]

531. Ishii T, Tatsuda E, Kumazawa S, Nakayama T, Uchida K. Molecular Basis of Enzyme Inactivation by sn Endogenous Electrophile 4-Hydroxy-2-nonenal: Identification of Modification Sites in Glyceraldehyde-3-Phosphate Dehydrogenase. Biochemistry. 2003; 42:3474-3480. [PubMed: 12653551]

532. Crabb JW, O'neil J, Miyagi M, West K, Hoff HF. Hydroxynonenal Inactivates Cathepsin B by Forming Michael Adducts with Active Site Residues. Protein Sci. 2002; 11:831-840. [PubMed: 11910026]

533. Bruns CM, Hubatsch I, Ridderstrom M, Mannervik B, Tainer JA. Human Glutathione Transferase A4-4 Crystal Structures and Mutagenesis Reveal the Basis of High Catalytic Efficiency with Toxic Lipid Peroxidation Products. J Mol Biol. 1999; 288:427-439. [PubMed: 10329152] 
534. Shireman LM, Kripps KA, Balogh LM, Conner KP, Whittington D, Atkins WM. Glutathione Transferase A4-4 Resists Adduction by 4-Hydroxynonenal. Arch Biochem Biophys. 2010; 504:182-189. [PubMed: 20836986]

535. Shibata T, Shimozu Y, Wakita C, Shibata N, Kobayashi M, Machida S, Kato R, Itabe H, Zhu X, Sayre LM, et al. Lipid Peroxidation Modification of Protein Generates Nepsilon-(4oxononanoyl)lysine as a Pro-Inflammatory Ligand. J Biol Chem. 2011; 286:19943-19957. [PubMed: 21471194]

536. Jin J, He B, Zhang X, Lin H, Wang Y. SIRT2 Reverses 4-Oxononanoyl Lysine Modification on Histones. J Am Chem Soc. 2016; 138:12304-12307. [PubMed: 27610633]

537. Yang J, Tallman KA, Porter NA, Liebler DC. Quantitative Chemoproteomics for Site-Specific Analysis of Protein Alkylation by 4-Hydroxy-2-nonenal in Cells. Anal Chem. 2015; 87:25352541. [PubMed: 25654326]

538. Requena JR, Fu MX, Ahmed MU, Jenkins AJ, Lyons TJ, Baynes JW, Thorpe SR. Quantification of Malondialdehyde and 4-Hydroxynonenal Adducts to Lysine Residues in Native and Oxidized Human Low-Density Lipoprotein. Biochem J. 1997; 322:317-325. [PubMed: 9078279]

539. Orioli M, Aldini G, Benfatto MC, Maffei Facino R, Carini M. HNE Michael Adducts to Histidine and Histidine-Containing Peptides as Biomarkers of Lipid-Derived Carbonyl Stress in Urines:w LC-MS/MS Profiling in Zucker Obese Rats. Anal Chem. 2007; 79:9174-9184. [PubMed: 17979257]

540. Fukuda M, Kanou F, Shimada N, Sawabe M, Saito Y, Murayama S, Hashimoto M, Maruyama N, Ishigami A. Elevated Levels of 4-Hydroxynonenal-Histidine Michael Adduct in the Hippocampi of Patients with Alzheimer's Disease. Biomed Res. 2009; 30:227-233. [PubMed: 19729853]

541. Labenski MT, Fisher AA, Lo HH, Monks TJ, Lau SS. Protein Electrophile-Binding Motifs: Lysine-Rich Proteins are Preferential Targets of Quinones. Drug Metab Disposition. 2009; 37:1211-1218.

542. Lau SS, Hill BA, Highet RJ, Monks TJ. Sequential Oxidation and Glutathione Addition to 1,4Benzoquinone: Correlation of Toxicity with Increased Glutathione Substitution. Mol Pharmacol. 1988; 34:829-836. [PubMed: 3200250]

543. Li Y, Jongberg S, Andersen ML, Davies MJ, Lund MN. Quinone-Induced Protein Modifications: Kinetic Preference for Reaction of 1,2-Benzoquinones with Thiol Groups in Proteins. Free Radical Biol Med. 2016; 97:148-157. [PubMed: 27212016]

544. Huber RE, Criddle RS. Comparison of the Chemical Properties of Selenocysteine and Selenocystine with their Sulfur Analogs. Arch Biochem Biophys. 1967; 122:164-173. [PubMed: 6076213]

545. Kryukov GV, Castellano S, Novoselov SV, Lobanov AV, Zehtab O, Guigó R, Gladyshev VN. Characterization of Mammalian Selenoproteomes. Science. 2003; 300:1439-1443. [PubMed: 12775843]

546. Arnér ESJ. Selenoproteins-What Unique Properties Can Arise with Selenocysteine in Place of Cysteine? Exp Cell Res. 2010; 316:1296-1303. [PubMed: 20206159]

547. Reich HJ, Hondal RJ. Why Nature Chose Selenium. ACS Chem Biol. 2016; 11:821-841. [PubMed: 26949981]

548. Wada M, Nobuki SI, Tenkyuu Y, Natsume S, Asahara M, Erabi T. Bis(2,6-dimethoxyphenyl) Sulfide, Selenide and Telluride, and Their Derivatives. J Organomet Chem. 1999; 580:282-289.

549. Orian L, Mauri P, Roveri A, Toppo S, Benazzi L, Bosello-Travain V, De Palma A, Maiorino M, Miotto G, Zaccarin M, et al. Selenocysteine Oxidation in Glutathione Peroxidase Catalysis: An MS-Supported Quantum Mechanics Study. Free Radical Biol Med. 2015; 87:1-14. [PubMed: 26163004]

550. Spiess PC, Deng B, Hondal RJ, Matthews DE, Van Der Vliet A. Proteomic Profiling of Acrolein Adducts in Human Lung Epithelial Cells. J Proteomics. 2011; 74:2380-2394. [PubMed: 21704744]

551. Randall MJ, Hristova M, Van Der Vliet A. Protein Alkylation by the $a, \beta$-Unsaturated Aldehyde Acrolein. A Reversible Mechanism of Electrophile Signaling? FEBS Lett. 2013; 587:3808-3814. [PubMed: 24157358] 
552. Von Ragué Schleyer P, Kos AJ. The Importance of Negative (Anionic) Hyperconjugation. Tetrahedron. 1983; 39:1141-1150.

553. Flemer S Jr. Selenol Protecting Groups in Organic Chemistry: Special Emphasis on Selenocysteine Se-Protection in Solid Phase Peptide Synthesis. Molecules. 2011; 16:3232. [PubMed: 21512438]

554. Karpinets TV, Greenwood DJ, Sams CE, Ammons JT. RNA:Protein Ratio of the Unicellular Organism as a Characteristic of Phosphorous and Nitrogen Stoichiometry and of the Cellular Requirement of Ribosomes for Protein Synthesis. BMC Biol. 2006; 4:30. [PubMed: 16953894]

555. Polakis ES, Bartley W. Changes in Dry Weight, Protein, Deoxyribonucleic Acid, Ribonucleic Acid and Reserve and Structural Carbohydrate During Aerobic Growth Cycle of Yeast. Biochem J. 1966; 98:883-887. [PubMed: 5911532]

556. Lauhon CT. Orchestrating Sulfur Incorporation into RNA. Nat Chem Biol. 2006; 2:182-183. [PubMed: 16547479]

557. Gomez M, Pérez-Gallardo RV, Sánchez LA, Díaz-Pérez AL, Cortés-Rojo C, Meza Carmen V, Saavedra-Molina A, Lara-Romero J, Jiménez-Sandoval S, Rodríguez F, et al. Malfunctioning of the Iron-Sulfur Cluster Assembly Machinery in Saccharomyces Cerevisiae Produces Oxidative Stress Via an Iron-Dependent Mechanism, Causing Dysfunction in Respiratory Complexes. PLoS One. 2014; 9:e111585. [PubMed: 25356756]

558. Outten FW. Iron-Sulfur Clusters as Oxygen-Responsive Molecular Switches. Nat Chem Biol. 2007; 3:206-207. [PubMed: 17372605]

559. Alhebshi A, Sideri TC, Holland SL, Avery SV. The Essential Iron-Sulfur Protein Rli1 is an Important Target Accounting for Inhibition of Cell Growth by Reactive Oxygen Species. Mol Biol Cell. 2012; 23:3582-3590. [PubMed: 22855532]

560. Hashimoto F, Hayashi H. Significance of catalase in peroxisomal fatty acyl-CoA beta-oxidation: NADH oxidation by acetoacetyl-CoA and H2O2. J Biochem. 1990; 108:426-431. [PubMed: 2277034]

561. Dizdaroglu M, Jaruga P. Mechanisms of Free Radical-Induced Damage to DNA. Free Radic Res. 2012; 46:382-419. [PubMed: 22276778]

562. Stubbe J, Kozarich JW. Mechanisms of Bleomycin-Induced Dna Degradation. Chem Rev. 1987; 87:1107-1136.

563. Nunomura A, Perry G, Pappolla MA, Wade R, Hirai K, Chiba S, Smith MA. RNA Oxidation is a Prominent Feature of Vulnerable Neurons in Alzheimer's Disease. J Neurosci. 1999; 19:19591964. [PubMed: 10066249]

564. Summerfield FW, Tappel AL. Determination of Malondialdehyde-DNA Crosslinks by Fluorescence and Incorporation of Tritium. Anal Biochem. 1981; 111:77-82. [PubMed: 7235243]

565. Choudhury S, Pan J, Amin S, Chung FL, Roy R. Repair Kinetics of trans-4-HydroxynonenalInduced Cyclic 1,N2-Propanodeoxyguanine DNA Adducts by Human Cell Nuclear Extracts. Biochemistry. 2004; 43:7514-7521. [PubMed: 15182193]

566. Levine RL, Yang IY, Hossain M, Pandya GA, Grollman AP, Moriya M. Mutagenesis Induced by a Single 1,N6-Ethenodeoxyadenosine Adduct in Human Cells. Cancer Res. 2000; 60:4098-4104. [PubMed: 10945616]

567. Huang H, Wang H, Qi N, Lloyd RS, Rizzo CJ, Stone MP. The Stereochemistry of Trans-4Hydroxynonenal-Derived Exocyclic 1,N2-2' -Deoxyguanosine Adducts Modulates Formation of Interstrand Cross-Links in the $5^{\prime}{ }^{-}$CpG-3' Sequence. Biochemistry. 2008; 47:11457-11472. [PubMed: 18847226]

568. Chung FL, Komninou D, Zhang L, Nath R, Pan J, Amin S, Richie J. Glutathione Depletion Enhances the Formation of Endogenous Cyclic DNA Adducts Derived from T-4-Hydroxy-2Nonenal in Rat Liver. Chem Res Toxicol. 2005; 18:24-27. [PubMed: 15651845]

569. Singh SP, Chen T, Chen L, Mei N, Mclain E, Samokyszyn V, Thaden JJ, Moore MM, Zimniak P. Mutagenic Effects of 4-Hydroxynonenal Triacetate, a Chemically Protected form of the Lipid Peroxidation Product 4-Hydroxynonenal, as Assayed in L5178Y/Tk+/- Mouse Lymphoma Cells. J Pharmacol Exp Ther. 2005; 313:855-861. [PubMed: 15701709] 
570. Linhart KB, Glassen K, Peccerella T, Waldherr R, Linhart H, Bartsch H, Seitz HK. The Generation of Carcinogenic Etheno-DNA Adducts in the Liver of Patients with Nonalcoholic Fatty Liver Disease. Hepatobiliary Surg Nutr. 2015; 4:117-123. [PubMed: 26005678]

571. Lin D, Lee HG, Liu Q, Perry G, Smith MA, Sayre LM. 4-Oxo-2-nonenal is Both More Neurotoxic and More Protein Reactive than 4-Hydroxy-2-nonenal. Chem Res Toxicol. 2005; 18:1219. [PubMed: 16097795]

572. Zhu P, Lee SH, Wehrli S, Blair IA. Characterization of a Lipid Hydroperoxide-Derived RNA Adduct in Rat Intestinal Epithelial Cells. Chem Res Toxicol. 2006; 19:809-817. [PubMed: 16780360]

573. Lee SH, Blair IA. Characterization of 4-Oxo-2-nonenal as a Novel Product of Lipid Peroxidation. Chem Res Toxicol. 2000; 13:698. [PubMed: 10956056]

574. Chen JJ, Bertrand H, Yu BP. Inhibition of Adenine Nucleotide Translocator by Lipid Peroxidation Products. Free Radical Biol Med. 1995; 19:583-590. [PubMed: 8529917]

575. Vander Jagt DL, Hunsaker LA, Vander Jagt TJ, Gomez MS, Gonzales DM, Deck LM, Royer RE. Inactivation of Glutathione Reductase by 4-Hydroxynonenal and Other Endogenous Aldehydes. Biochem Pharmacol. 1997; 53:1133-1140. [PubMed: 9175718]

576. Gabriel L, Miglietta A, Dianzani MU. 4-Hydroxy-Alkenals Interaction with Purified Microtubular Protein. Chem-Biol Interact. 1985; 56:201-212. [PubMed: 4075448]

577. Barlow R. Enantiomers: How Valid is Pfeiffer's Rule? Trends Pharmacol Sci. 1990; 11:148-150. [PubMed: 2333666]

578. Dabrowski MJ, Zolnerciks JK, Balogh LM, Greene RJ, Kavanagh TJ, Atkins WM. Stereoselective Effects of 4-Hydroxynonenal in Cultured Mouse Hepatocytes. Chem Res Toxicol. 2010; 23:1601-1607. [PubMed: 20873854]

579. Wakita C, Maeshima T, Yamazaki A, Shibata T, Ito S, Akagawa M, Ojika M, Yodoi J, Uchida K. Stereochemical Configuration of 4-Hydroxy-2-nonenal-Cysteine Adducts and their Stereoselective Formation in a Redox-Regulated Protein. J Biol Chem. 2009; 284:28810-28822. [PubMed: 19692331]

580. Van Bergen LaHRoos G, De Proft F. From Thiol to Sulfonic Acid: Modeling the Oxidation Pathway of Protein Thiols by Hydrogen Peroxide. J Phys Chem A. 2014; 118:6078-6084. [PubMed: 25036614]

581. Lopachin RM, Gavin T, Petersen DR, Barber DS. Molecular Mechanisms of 4-Hydroxy-2nonenal and Acrolein Toxicity: Nucleophilic Targets and Adduct Formation. Chem Res Toxicol. 2009; 22:1499-1508. [PubMed: 19610654]

582. Perkins A, Parsonage D, Nelson KJ, Ogba OM, Cheong PH, Poole LB, Karplus PA. Peroxiredoxin Catalysis at Atomic Resolution. Structure. 2016; 24:1668-1678. [PubMed: 27594682]

583. Karplus PA. A Primer on Peroxiredoxin Biochemistry. Free Radical Biol Med. 2015; 80:183-190. [PubMed: 25452140]

584. Hall A, Karplus PA, Poole LB. Typical 2-Cys Peroxiredoxins--Structures, Mechanisms and Functions. FEBS J. 2009; 276:2469-2477. [PubMed: 19476488]

585. Caccuri AM, Antonini G, Nicotra M, Battistoni A, Lo Bello M, Board PG, Parker MW, Ricci G. Catalytic Mechanism and Role Of Hydroxyl Residues in the Active Site of Theta Class Glutathione S-Transferases. Investigation of Ser-9 and Tyr-113 in a Glutathione S-Transferase from the Australian Sheep Blowfly, Lucilia cuprina. J Biol Chem. 1997; 272:29681-29686. [PubMed: 9368035]

586. Deponte M. Glutathione Catalysis and the Reaction Mechanisms of Glutathione-Dependent Enzymes. Biochim Biophys Acta. 2013; 1830:3217-3266. [PubMed: 23036594]

587. Lohman DC, Edwards DR, Wolfenden R. Catalysis by Desolvation: The Catalytic Prowess of SAM-Dependent Halide-Alkylating Enzymes. J Am Chem Soc. 2013; 135:14473-14475. [PubMed: 24041082]

588. Hall A, Parsonage D, Poole LB, Karplus PA. Structural Evidence that Peroxiredoxin Catalytic Power is Based on Transition-State Stabilization. J Mol Biol. 2010; 402:194-209. [PubMed: 20643143] 
589. Sanchez R, Riddle M, Woo J, Momand J. Prediction of Reversibly Oxidized Protein Cysteine Thiols Using Protein Structure Properties. Protein Sci. 2008; 17:473-481. [PubMed: 18287280]

590. Sun MA, Zhang Q, Wang Y, Ge W, Guo D. Prediction of Redox-Sensitive Cysteines Using Sequential Distance and Other Sequence-Based Features. BMC Bioinformatics. 2016; 17:316. [PubMed: 27553667]

591. Ho MC, Menetret JF, Tsuruta H, Allen KN. The Origin of the Electrostatic Perturbation in Acetoacetate Decarboxylase. Nature. 2009; 459:393-397. [PubMed: 19458715]

592. Laursen RA, Westheimer FH. The Active Site of Acetoacetate Decarboxylase1. J Am Chem Soc. 1966; 88:3426-3430. [PubMed: 5946601]

593. Harris TK, Turner GJ. Structural Basis of Perturbed pKa Values of Catalytic Groups in Enzyme Active Sites. IUBMB Life. 2002; 53:85-98. [PubMed: 12049200]

594. Britto PJ, Knipling L, Wolff J. The Local Electrostatic Environment Determines Cysteine Reactivity of Tubulin. J Biol Chem. 2002; 277:29018-29027. [PubMed: 12023292]

595. Snyder GH, Cennerazzo MJ, Karalis AJ, Locey D. Electrostatic Influence of Local Cysteine Environments on Disulfide Exchange Kinetics. Biochemistry. 1981; 20:6509-6519. [PubMed: 6796114]

596. Bocedi A, Fabrini R, Pedersen JZ, Federici G, Iavarone F, Martelli C, Castagnola M, Ricci G. The Extreme Hyper-Reactivity of Selected Cysteines Drives Hierarchical Disulfide Bond Formation in Serum Albumin. FEBS J. 2016; 283:4113-4127. [PubMed: 27685835]

597. Witt AC, Lakshminarasimhan M, Remington BC, Hasim S, Pozharski E, Wilson MA. Cysteine pK(a) Depression by a Protonated Glutamic Acid in Human DJ-1. Biochemistry. 2008; 47:7430 7440. [PubMed: 18570440]

598. Kortemme T, Creighton TE. Ionisation of Cysteine Residues at the Termini of Model AlphaHelical Peptides. Relevance to Unusual Thiol pKa Values in Proteins of the Thioredoxin Family. J Mol Biol. 1995; 253:799-812. [PubMed: 7473753]

599. Chivers PT, Prehoda KE, Volkman BF, Kim BM, Markley JL, Raines RT. Microscopic pKa Values of Escherichia coli Thioredoxin. Biochemistry. 1997; 36:14985-14991. [PubMed: 9398223]

600. Jao SC, English Ospina SM, Berdis AJ, Starke DW, Post CB, Mieyal JJ. Computational and Mutational Analysis of Human Glutaredoxin (Thioltransferase): Probing the Molecular Basis of the Low pKa of Cysteine 22 and its Role in Catalysis. Biochemistry. 2006; 45:4785-4796. [PubMed: 16605247]

601. Hennecke J, Spleiss C, Glockshuber R. Influence of Acidic Residues and the Kink in the ActiveSite Helix on the Properties of the Disulfide Oxidoreductase DsbA. J Biol Chem. 1997; 272:189195. [PubMed: 8995246]

602. Madzelan P, Labunska T, Wilson MA. Influence of Peptide Dipoles and Hydrogen Bonds on Reactive Cysteine pKa Values in Fission Yeast DJ-1. FEBS J. 2012; 279:4111-4120. [PubMed: 22971103]

603. Lim JC, Gruschus JM, Kim G, Berlett BS, Tjandra N, Levine RL. A Low pK(a) Cysteine at the Active Site of Mouse Methionine Sulfoxide Reductase A. J Biol Chem. 2012; 287:25596-25601. [PubMed: 22661719]

604. Mazmanian K, Sargsyan K, Grauffel C, Dudev T, Lim C. Preferred Hydrogen-Bonding Partners of Cysteine: Implications for Regulating Cys Functions. J Phys Chem B. 2016; 120:1028810296. [PubMed: 27635780]

605. Nath A, Atkins WM. A Quantitative Index of Substrate Promiscuity. Biochemistry. 2008; 47:157166. [PubMed: 18081310]

606. Graminski GF, Kubo Y, Armstrong RN. Spectroscopic and Kinetic Evidence for the Thiolate Anion of Glutathione at the Active Site of Glutathione S-Transferase. Biochemistry. 1989; 28:3562-3568. [PubMed: 2742854]

607. Bryan HK, Olayanju A, Goldring CE, Park BK. The Nrf2 Cell Defence Pathway: Keap1Dependent and -Independent Mechanisms of Regulation. Biochem Pharmacol. 2013; 85:705717. [PubMed: 23219527] 
608. Dinkova-Kostova AT, Kostov RV, Canning P. Keap1, the Cysteine-Based Mammalian Intracellular Sensor for Electrophiles and Oxidants. Arch Biochem Biophys. 2017; 617:84-93. [PubMed: 27497696]

609. Lin HY, Haegele JA, Disare MT, Lin Q, Aye Y. A Generalizable Platform for Interrogating Targetand Signal-Specific Consequences of Electrophilic Modifications in Redox-Dependent Cell Signaling. J Am Chem Soc. 2015; 137:6232-6244. [PubMed: 25909755]

610. Kumar MR, Farmer PJ. Trapping Reactions of the Sulfenyl and Sulfinyl Tautomers of Sulfenic Acids. ACS Chem Biol. 2017; 12:474-478. [PubMed: 27984696]

611. Poole LB, Karplus PA, Claiborne A. Protein Sulfenic Acids in Redox Signaling. Annu Rev Pharmacol Toxicol. 2004; 44:325-347. [PubMed: 14744249]

612. Kettenhofen NJ, Wood MJ. Formation, Reactivity and Detection of Protein Sulfenic Acids. Chem Res Toxicol. 2010; 23:1633-1646. [PubMed: 20845928]

613. Florence TM. Degradation of Protein Disulphide Bonds in Dilute Alkali. Biochem J. 1980; 189:507-520. [PubMed: 7213343]

614. Brandt W, Golbraikh A, Täger M, Lendeckel U. A Molecular Mechanism for the Cleavage of a Disulfide Bond as the Primary Function of Agonist Binding to G-Protein-Coupled Receptors Based on Theoretical Calculations Supported by Experiments. Eur J Biochem. 1999; 261:89-97. [PubMed: 10103038]

615. Allison WS, Benitez LV. An Adenosine Triphosphate-Phosphate Exchange Catalyzed by a Soluble Enzyme Couple Inhibited by Uncouplers of Oxidative Phosphorylation. Proc Natl Acad Sci U S A. 1972; 69:3004-3008. [PubMed: 4507619]

616. Percival MD, Ouellet M, Campagnolo C, Claveau D, Li C. Inhibition of Cathepsin K by Nitric Oxide Donors:w Evidence for the Formation of Mixed Disulfides and a Sulfenic Acid. Biochemistry. 1999; 38:13574-13583. [PubMed: 10521264]

617. Fraenkel-Conrat H. The Reaction of Tobacco Mosaic Virus with Iodine. J Biol Chem. 1955; 217:373-382. [PubMed: 13271401]

618. Fawcett DM. The Formation of Sulfenyl Iodides as Intermediates During the in Vitro Iodination of Tyrosine by Calf Thyroid Homogenates. Can J Biochem. 1968; 46:1433-1441. [PubMed: 5704375]

619. Kim Y, Jo H, Lim CJ. Deubiquitinating Activity of Sdu1, a Putative Member of the PPPDE Peptidase Family, in Schizosaccharomyces pombe. Can J Microbiol. 2013; 59:789-796. [PubMed: 24313451]

620. Komander D, Clague MJ, Urbé S. Breaking the Chains: Structure and Function of the Deubiquitinases. Nat Rev Mol Cell Biol. 2009; 10:550. [PubMed: 19626045]

621. Kulathu Y, Garcia FJ, Mevissen TET, Busch M, Arnaudo N, Carroll KS, Barford D, Komander D. Regulation of A20 and Other OTU Deubiquitinases by Reversible Oxidation. Nat Commun. 2013; 4:1569-1569. [PubMed: 23463012]

622. Devarie-Baez NO, Silva Lopez EI, Furdui CM. Biological Chemistry and Functionality of Protein Sulfenic Acids and Related Thiol Modifications. Free Radic Res. 2016; 50:172-194. [PubMed: 26340608]

623. Silva GM, Finley D, Vogel C. K63 Polyubiquitination is a New Modulator of the Oxidative Stress Response. Nat Struct Mol Biol. 2015; 22:116-123. [PubMed: 25622294]

624. Martinez S, Wu R, Sanishvili R, Liu D, Holz R. The Active Site Sulfenic Acid Ligand in Nitrile Hydratases Can Function as a Nucleophile. J Am Chem Soc. 2014; 136:1186-1189. [PubMed: 24383915]

625. Claiborne A, Yeh JI, Mallett TC, Luba J, Crane EJ, Charrier V, Parsonage D. Protein-Sulfenic Acids:w Diverse Roles for an Unlikely Player in Enzyme Catalysis and Redox Regulation. Biochemistry. 1999; 38:15407-15416. [PubMed: 10569923]

626. Boschi-Muller S, Azza S, Sanglier-Cianferani S, Talfournier F, Van Dorsselear A, Branlant G. A Sulfenic Acid Enzyme Intermediate is Involved in the Catalytic Mechanism of Peptide Methionine Sulfoxide Reductase From escherichia coli. J Biol Chem. 2000; 275:35908-35913. [PubMed: 10964927]

627. Fu X, Mueller DM, Heinecke JW. Generation of Intramolecular and Intermolecular Sulfenamides, Sulfinamides, and Sulfonamides by Hypochlorous Acid:w A Potential Pathway for Oxidative 
Cross-Linking of Low-Density Lipoprotein by Myeloperoxidase. Biochemistry. 2002; 41:12931301. [PubMed: 11802729]

628. Kang P, Dalvie D, Smith E, Zhou S, Deese A. Identification of a Novel Glutathione Conjugate of Flutamide in Incubations with Human Liver Microsomes. Drug Metab Dispos. 2007; 35:10811088. [PubMed: 17403914]

629. Allison WS, Benitez LV, Johnson CL. The Formation of a Protein Sulfenamide During the Inactivation of the Acyl Phosphatase Activity of Oxidized Glyceraldehyde-3-Phosphate Dehydrogenase by Benzylamine. Biochem Biophys Res Commun. 1973; 52:1403-1409. [PubMed: 4717755]

630. Van Montfort RLM, Congreve M, Tisi D, Carr R, Jhoti H. Oxidation State of the Active-Site Cysteine in Protein Tyrosine Phosphatase 1B. Nature. 2003; 423:773-777. [PubMed: 12802339]

631. Singh DK, Kumar D, Siddiqui Z, Basu SK, Kumar V, Rao KVS. The Strength of Receptor Signaling is Centrally Controlled Through a Cooperative Loop between Ca2+ and an Oxidant Signal. Cell. 2005; 121:281-293. [PubMed: 15851034]

632. Defelipe LA, Lanzarotti E, Gauto D, Marti MA, Turjanski AG. Protein Topology Determines Cysteine Oxidation Fate: The Case of Sulfenyl Amide Formation Among Protein Families. PLoS Comp Biol. 2015; 11:e1004051.

633. Yang J, Groen A, Lemeer S, Jans A, Slijper M, Roe SM, Den Hertog J, Barford D. Reversible Oxidation of the Membrane Distal Domain of Receptor PTPa is Mediated by a Cyclic Sulfenamide. Biochemistry. 2007; 46:709-719. [PubMed: 17223692]

634. Rodkey EA, Drawz SM, Sampson JM, Bethel CR, Bonomo RA, Van Den Akker F. Crystal Structure of a Preacylation Complex of the $\beta$-lactamase Inhibitor Sulbactam Bound to a Sulfenamide Bond-Containing Thiol- $\beta$-lactamase. J Am Chem Soc. 2012; 134:16798-16804. [PubMed: 22974281]

635. Gray MJ, Li Y, Leichert LIO, Xu Z, Jakob U. Does the Transcription Factor NemR Use a Regulatory Sulfenamide Bond to Sense Bleach? Antioxid Redox Signal. 2015; 23:747-754. [PubMed: 25867078]

636. Kim G, Levine RL. A Methionine Residue Promotes Hyperoxidation of the Catalytic Cysteine of Mouse Methionine Sulfoxide Reductase A. Biochemistry. 2016; 55:3586-3593. [PubMed: 27259041]

637. Lim JC, You Z, Kim G, Levine RL. Methionine Sulfoxide Reductase A is a Stereospecific Methionine Oxidase. Proc Natl Acad Sci U S A. 2011; 108:10472-10477. [PubMed: 21670260]

638. Kuehler TC, Fryklund J, Bergman NK, Weilitz J, Lee A, Larsson H. Structure-Activity Relationship of Omeprazole and Analogs as Helicobacter Pylori Urease Inhibitors. J Med Chem. 1995; 38:4906-4916. [PubMed: 8523404]

639. Bulleid NJ. Disulfide Bond Formation in the Mammalian Endoplasmic Reticulum. Cold Spring Harb Perspect Biol. 2012; 4:a013219. [PubMed: 23125019]

640. Butera D, Cook KM, Chiu J, Wong JWH, Hogg PJ. Control of Blood Proteins by Functional Disulfide Bonds. Blood. 2014; 123:2000-2007. [PubMed: 24523239]

641. Saaranen M, Ruddock L. Disulfide Bond Formation in the Cytoplasm. Antioxid Redox Signal. 2012; 19:46-53. [PubMed: 22870953]

642. Cumming RC, Andon NL, Haynes PA, Park M, Fischer WH, Schubert D. Protein Disulfide Bond Formation in the Cytoplasm During Oxidative Stress. J Biol Chem. 2004; 279:21749-21758. [PubMed: 15031298]

643. Wouters MA, Fan SW, Haworth NL. Disulfides as Redox Switches: From Molecular Mechanisms to Functional Significance. Antioxid Redox Signal. 2010; 12:53-91. [PubMed: 19634988]

644. Sun MA, Wang Y, Zhang Q, Xia Y, Ge W, Guo D. Prediction of Reversible Disulfide Based on Features from Local Structural Signatures. BMC Genomics. 2017; 18:279. [PubMed: 28376774]

645. Lee SR, Yang KS, Kwon J, Lee C, Jeong W, Rhee SG. Reversible Inactivation of the Tumor Suppressor PTEN by H2O2. J Biol Chem. 2002; 277:20336-20342. [PubMed: 11916965]

646. Kwon J, Lee SR, Yang KS, Ahn Y, Kim YJ, Stadtman ER, Rhee SG. Reversible Oxidation and Inactivation of the Tumor Suppressor PTEN in Cells Stimulated with Peptide Growth Factors. Proc Natl Acad Sci U S A. 2004; 101:16419-16424. [PubMed: 15534200] 
647. Caselli A, Marzocchini R, Camici G, Manao G, Moneti G, Pieraccini G, Ramponi G. The Inactivation Mechanism of Low Molecular Weight Phosphotyrosine-Protein Phosphatase by H2O2. J Biol Chem. 1998; 273:32554-32560. [PubMed: 9829991]

648. Sohn J, Rudolph J. Catalytic and Chemical Competence of Regulation of Cdc25 Phosphatase by Oxidation/Reduction. Biochemistry. 2003; 42:10060-10070. [PubMed: 12939134]

649. Buhrman G, Parker B, Sohn J, Rudolph J, Mattos C. Structural Mechanism of Oxidative Regulation of the Phosphatase Cdc25B Via an Intramolecular Disulfide Bond. Biochemistry. 2005; 44:5307-5316. [PubMed: 15807524]

650. Schwertassek U, Haque A, Krishnan N, Greiner R, Weingarten L, Dick TP, Tonks NK. Reactivation of Oxidized PTP1B and PTEN by Thioredoxin 1. FEBS J. 2014; 281:3545-3558. [PubMed: 24976139]

651. Dagnell M, Pace PE, Cheng Q, Frijhoff J, Ostman A, Arner ESJ, Hampton MB, Winterbourn CC. Thioredoxin Reductase 1 and Nadph Directly Protect Protein Tyrosine Phosphatase 1B from Inactivation During H2O2 Exposure. J Biol Chem. 2017; 292:14371-14380. [PubMed: 28684416]

652. Palde PB, Carroll KS. A Universal Entropy-Driven Mechanism for Thioredoxin-Target Recognition. Proc Natl Acad Sci U S A. 2015; 112:7960-7965. [PubMed: 26080424]

653. Verrastro I, Tveen-Jensen K, Woscholski R, Spickett CM, Pitt AR. Reversible Oxidation of Phosphatase and Tensin Homolog (PTEN) Alters its Interactions with Signaling and Regulatory Proteins. Free Radical Biol Med. 2016; 90:24-34. [PubMed: 26561776]

654. Hangen E, Blomgren K, Bénit P, Kroemer G, Modjtahedi N. Life With or Without AIF. Trends Biochem Sci. 2010; 35:278-287. [PubMed: 20138767]

655. Shen SM, Guo M, Xiong Z, Yu Y, Zhao XY, Zhang FF, Chen GQ. AIF Inhibits Tumor Metastasis by Protecting PTEN from Oxidation. EMBO Rep. 2015; 16:1563-1580. [PubMed: 26415504]

656. Dalle-Donne I, Rossi R, Colombo G, Giustarini D, Milzani A. Protein S-Glutathionylation: A Regulatory Device from Bacteria to Humans. Trends Biochem Sci. 2009; 34:85-96. [PubMed: 19135374]

657. Ghezzi P. Protein Glutathionylation in Health and Disease. Biochim Biophys Acta. 2013; 1830:3165-3172. [PubMed: 23416063]

658. Lillig CH, Berndt C. Glutaredoxins in Thiol/Disulfide Exchange. Antioxid Redox Signal. 2012; 18:1654-1665. [PubMed: 23231445]

659. Ghezzi P. Regulation of Protein Function by Glutathionylation. Free Radic Res. 2005; 39:573580. [PubMed: 16036334]

660. Wang J, Pareja KA, Kaiser CA, Sevier CS. Redox Signaling via the Molecular Chaperone BiP Protects Cells Against Endoplasmic Reticulum-Derived Oxidative Stress. eLife. 2014; 3:e03496. [PubMed: 25053742]

661. Wang J, Sevier CS. Formation and Reversibility of BiP Protein Cysteine Oxidation Facilitate Cell Survival During and Post Oxidative Stress. J Biol Chem. 2016; 291:7541-7557. [PubMed: 26865632]

662. Watanabe Y, Murdoch CE, Sano S, Ido Y, Bachschmid MM, Cohen RA, Matsui R. Glutathione Adducts Induced by Ischemia and Deletion of Glutaredoxin-1 Stabilize HIF-1a and Improve Limb Revascularization. Proc Natl Acad Sci U S A. 2016; 113:6011-6016. [PubMed: 27162359]

663. Adachi T, Weisbrod RM, Pimentel DR, Ying J, Sharov VS, Schöneich C, Cohen RA. SGlutathiolation by Peroxynitrite Activates SERCA During Arterial Relaxation by Nitric Oxide. Nat Med. 2004; 10:1200. [PubMed: 15489859]

664. Tsuchiya Y, Peak-Chew SY, Newell C, Miller-Aidoo S, Mangal S, Zhyvoloup A, Baković J, Malanchuk O, Pereira GC, Kotiadis V, et al. Protein CoAlation: A Redox-Regulated Protein Modification by Coenzyme A in Mammalian Cells. Biochem J. 2017; 474:2489-2508. [PubMed: 28341808]

665. Leonardi R, Zhang YM, Rock CO, Jackowski S. Coenzyme A: Back in Action. Prog Lipid Res. 2005; 44:125-153. [PubMed: 15893380]

666. Longen S, Richter F, Köhler Y, Wittig I, Beck KF, Pfeilschifter J. Quantitative Persulfide Site Identification (qPerS-SID) Reveals Protein Targets of H2S Releasing Donors in Mammalian Cells. Sci Rep. 2016; 6:29808. [PubMed: 27411966] 
667. Rickey Welch G, Easterby JS. Metabolic Channeling Versus Free Diffusion: Transition-Time Analysis. Trends Biochem Sci. 1994; 19:193-197. [PubMed: 8048159]

668. Delaunay A, Pflieger D, Barrault MB, Vinh J, Toledano MB. A Thiol Peroxidase is an H2O2 Receptor and Redox-Transducer in Gene Activation. Cell. 2002; 111:471-481. [PubMed: 12437921]

669. Vivancos AP, Castillo EA, Biteau B, Nicot C, Ayte J, Toledano MB, Hidalgo E. A CysteineSulfinic Acid in Peroxiredoxin Regulates H2O2-Sensing by the Antioxidant Pap1 Pathway. Proc Natl Acad Sci U S A. 2005; 102:8875-8880. [PubMed: 15956211]

670. Sobotta MC, Liou W, Stöcker S, Talwar D, Oehler M, Ruppert T, Scharf AND, Dick TP. Peroxiredoxin-2 and STAT3 Form a Redox Relay for H2O2 Signaling. Nat Chem Biol. 2014; 11:64-70. [PubMed: 25402766]

671. Brigelius-Flohe R, Flohe L. Basic Principles and Emerging Concepts in the Redox Control of Transcription Factors. Antioxid Redox Signal. 2011; 15:2335-2381. [PubMed: 21194351]

672. Hung RJ, Pak CW, Terman JR. Direct Redox Regulation of F-actin Assembly and Disassembly by Mical. Science. 2011; 334:1710-1713. [PubMed: 22116028]

673. Veredas FJ, Cantón FR, Aledo JC. Methionine Residues Around Phosphorylation Sites are Preferentially Oxidized in Vivo Under Stress Conditions. Sci Rep. 2017; 7:40403. [PubMed: 28079140]

674. Reddy VY, Desorchers PE, Pizzo SV, Gonias SL, Sahakian JA, Levine RL, Weiss SJ. Oxidative Dissociation of Human Alpha 2-Macroglobulin Tetramers into Dysfunctional Dimers. J Biol Chem. 1994; 269:4683-4691. [PubMed: 7508448]

675. Levine RL, Mosoni L, Berlett BS, Stadtman ER. Methionine Residues as Endogenous Antioxidants inwProteins. Proc Natl Acad Sci U S A. 1996; 93:15036-15040. [PubMed: 8986759]

676. Luo S, Levine RL. Methionine in Proteins Defends Against Oxidative Stress. FASEB J. 2009; 23:464-472. [PubMed: 18845767]

677. Erickson JR, Joiner M-LA, Guan X, Kutschke W, Yang J, Oddis CV, Bartlett RK, Lowe JS, O'donnell S, Aykin-Burns N, et al. A Dynamic Pathway for Calcium-Independent Activation of CaMKII by Methionine Oxidation. Cell. 2008; 133:462-474. [PubMed: 18455987]

678. Snijder J, Rose RJ, Raijmakers R, Heck AJR. Site-Specific Methionine Oxidation in Calmodulin Affects Structural Integrity and Interaction with $\mathrm{Ca} 2+/$ Calmodulin-Dependent Protein Kinase II. J Struct Biol. 2011; 174:187-195. [PubMed: 21156208]

679. Townsend D, Hughes E, Hussain R, Siligardi G, Baldock S, Madine J, Middleton DA. Heparin and Methionine Oxidation Promote the Formation of Apolipoprotein A-I Amyloid Comprising $a-H e l i c a l$ and $\beta$-Sheet Structures. Biochemistry. 2017; 56:1632-1644. [PubMed: 27992182]

680. Carruthers NJ, Stemmer PM. Methionine Oxidation in the Calmodulin-Binding Domain of Calcineurin Disrupts Calmodulin Binding and Calcineurin Activation. Biochemistry. 2008; 47:3085-3095. [PubMed: 18275158]

681. Jeong W, Park SJ, Chang TS, Lee DY, Rhee SG. Molecular Mechanism of the Reduction of Cysteine Sulfinic Acid of Peroxiredoxin to Cysteine by Mammalian Sulfiredoxin. J Biol Chem. 2006; 281:14400-14407. [PubMed: 16565085]

682. Andres-Mateos E, Perier C, Zhang L, Blanchard-Fillion B, Greco TM, Thomas B, Ko HS, Sasaki M, Ischiropoulos H, Przedborski S, et al. DJ-1 Gene Deletion Reveals That DJ-1 is an Atypical Peroxiredoxin-Like Peroxidase. Proc Natl Acad Sci U S A. 2007; 104:14807-14812. [PubMed: 17766438]

683. Canet-Avilés RM, Wilson MA, Miller DW, Ahmad R, Mclendon C, Bandyopadhyay S, Baptista MJ, Ringe D, Petsko GA, Cookson MR. The Parkinson's Disease Protein DJ-1 is Neuroprotective due to Cysteine-Sulfinic Acid-Driven Mitochondrial Localization. Proc Natl Acad Sci U S A. 2004; 101:9103-9108. [PubMed: 15181200]

684. Blackinton J, Lakshminarasimhan M, Thomas KJ, Ahmad R, Greggio E, Raza AS, Cookson MR, Wilson MA. Formation of a Stabilized Cysteine Sulfinic Acid is Critical for the Mitochondrial Function of the Parkinsonism Protein DJ-1. J Biol Chem. 2009; 284:6476-6485. [PubMed: 19124468] 
685. Murakami T, Nojiri M, Nakayama H, Odaka M, Yohda M, Dohmae N, Takio K, Nagamune T, Endo I. Post-Translational Modification is Essential for Catalytic Activity of Nitrile Hydratase. Protein Sci. 2000; 9:1024-1030. [PubMed: 10850812]

686. Fu X, Kassim SY, Parks WC, Heinecke JW. Hypochlorous Acid Oxygenates the Cysteine Switch Domain of Pro-Matrilysin (MMP-7): a Mechanism for Matrix Metalloproteinase Activation and Atherosclerotic Plaque Rupture by Myeloperoxidase. J Biol Chem. 2001; 276:41279-41287. [PubMed: 11533038]

687. Friguet B. Oxidized Protein Degradation and Repair in Ageing and Oxidative Stress. FEBS Lett. 2006; 580:2910-2916. [PubMed: 16574110]

688. Tasaki T, Sriram SM, Park KS, Kwon YT. The N-End Rule Pathway. Annu Rev Biochem. 2012; 81:261-289. [PubMed: 22524314]

689. Chen S-J, Wu X, Wadas B, Oh J-H, Varshavsky A. An N-End Rule Pathway that Recognizes Proline and Destroys Gluconeogenic Enzymes. Science. 2017:355. [PubMed: 28126774]

690. Grzegorski SJ, Chiari EF, Robbins A, Kish PE, Kahana A. Natural Variability of Kozak Sequences Correlates with Function in a Zebrafish Model. PLoS One. 2014; 9:e108475. [PubMed: 25248153]

691. Forte GMA, Pool MR, Stirling CJ. N-Terminal Acetylation Inhibits Protein Targeting to the Endoplasmic Reticulum. PLoS Biol. 2011; 9:e1001073. [PubMed: 21655302]

692. Giglione C, Boularot A, Meinnel T. Protein N-Terminal Methionine Excision. Cell Mol Life Sci. 2004; 61:1455-1474. [PubMed: 15197470]

693. Laemmli UK. Cleavage of Structural Proteins During the Assembly of the Head of Bacteriophage T4. Nature. 1970; 227:680. [PubMed: 5432063]

694. Hu RG, Sheng J, Qi X, Xu Z, Takahashi TT, Varshavsky A. The N-end Rule Pathway as a Nitric Oxide Sensor Controlling the Levels of Multiple Regulators. Nature. 2005; 437:981. [PubMed: 16222293]

695. Lim JC, Choi HI, Park YS, Nam HW, Woo HA, Kwon KS, Kim YS, Rhee SG, Kim K, Chae HZ. Irreversible Oxidation of the Active-Site Cysteine of Peroxiredoxin to Cysteine Sulfonic Acid for Enhanced Molecular Chaperone Activity. J Biol Chem. 2008; 283:28873-28880. [PubMed: 18725414]

696. Fujiwara N, Nakano M, Kato S, Yoshihara D, Ookawara T, Eguchi H, Taniguchi N, Suzuki K. Oxidative Modification To Cysteine Sulfonic Acid of Cys111 in Human Copper-Zinc Superoxide Dismutase. J Biol Chem. 2007; 282:35933-35944. [PubMed: 17913710]

697. Chen X, Shang H, Qiu X, Fujiwara N, Cui L, Li XM, Gao TM, Kong J. Oxidative Modification of Cysteine 111 Promotes Disulfide Bond-Independent Aggregation of SOD1. Neurochem Res. 2012; 37:835-845. [PubMed: 22219129]

698. Kelley EE, Batthyany CI, Hundley NJ, Woodcock SR, Bonacci G, Del Rio JM, Schopfer FJ, Lancaster JR, Freeman BA, Tarpey MM. Nitro-oleic Acid, a Novel and Irreversible Inhibitor of Xanthine Oxidoreductase. J Biol Chem. 2008; 283:36176-36184. [PubMed: 18974051]

699. Rubbo H, Gonzalez-Perilli L, Mastrogiovanni M, Sánchez-Calvo B, Trostchansky A. Biochemistry of oxidative stress. Gelpi RJ, Boveris A, Poderoso JJ, editorsCham: 2016.

700. González-Perilli L, Mastrogiovanni M, De Castro Fernandes D, Rubbo H, Laurindo F, Trostchansky A. Nitroarachidonic Acid (NO2AA) Inhibits Protein Disulfide Isomerase (PDI) Through Reversible Covalent Adduct Formation with Critical Cysteines. Biochim Biophys Acta. 2017; 1861:1131-1139.

701. Jirošova A, Jančařik A, Menezes RC, Bazalova O, Dolejšova K, Vogel H, Jedlička P, Buček A, Brabcova J, Majer P, et al. Co-option of the Sphingolipid Metabolism for the Production of Nitroalkene Defensive Chemicals in Termite Soldiers. Insect Biochem Mol Biol. 2017; 82:52-61. [PubMed: 28126587]

702. Akashi S, Ahmed KA, Sawa T, Ono K, Tsutsuki H, Burgoyne JR, Ida T, Horio E, Prysyazhna O, Oike Y, et al. Persistent Activation of cGMP-Dependent Protein Kinase by a Nitrated Cyclic Nucleotide via Site Specific Protein S-Guanylation. Biochemistry. 2016; 55:751-761. [PubMed: 26784639] 
703. Francis SH, Busch JL, Corbin JD. cGMP-Dependent Protein Kinases and cGMP Phosphodiesterases in Nitric Oxide and cGMP Action. Pharmacol Rev. 2010; 62:525-563. [PubMed: 20716671]

704. Wang N, Wang Y, Yu G, Yuan C, Ma J. Quinoprotein Adducts Accumulate in the Substantia Nigra of Aged Rats and Correlate with Dopamine-Induced Toxicity in sh-sy5y Cells. Neurochem Res. 2011; 36:2169. [PubMed: 21785836]

705. Yu G, Liu H, Zhou W, Zhu X, Yu C, Wang N, Zhang Y, Ma J, Zhao Y, Xu Y, et al. In Vivo Protein Targets for Increased Quinoprotein Adduct Formation in Aged Substantia Nigra. Exp Neurol. 2015; 271:13-24. [PubMed: 25956828]

706. Belluzzi E, Bisaglia M, Lazzarini E, Tabares LC, Beltramini M, Bubacco L. Human SOD2 Modification by Dopamine Quinones Affects Enzymatic Activity by Promoting its Aggregation: Possible Implications for Parkinson's Disease. PLoS One. 2012; 7:e38026. [PubMed: 22723845]

707. Scott CK, Sundell E, Castrovilly L. Studies on the Mechanism of Action of the Gastric Microsomal (H+ + K+)-ATPase Inhibitors SCH 32651 and SCH 28080. Biochem Pharmacol. 1987; 36:97-104. [PubMed: 3026407]

708. Chen W, Koenigs LL, Thompson SJ, Peter RM, Rettie AE, Trager WF, Nelson SD. Oxidation of Acetaminophen to its Toxic Quinone Imine and Nontoxic Catechol Metabolites by BaculovirusExpressed and Purified Human Cytochromes P450 2E1 and 2A6. Chem Res Toxicol. 1998; 11:295-301. [PubMed: 9548799]

709. Mchale CM, Zhang L, Smith MT. Current Understanding of the Mechanism of Benzene-Induced Leukemia in Humans: Implications for Risk Assessment. Carcinogenesis. 2012; 33:240-252. [PubMed: 22166497]

710. Hukkanen J. Induction of Cytochrome P450 Enzymes: A View on Human in Vivo Findings. Expert Rev Clin Pharmacol. 2012; 5:569-585. [PubMed: 23121279]

711. Burczynski ME, Lin HK, Penning TM. Isoform-Specific Induction of a Human Aldo-Keto Reductase by Polycyclic Aromatic Hydrocarbons (PAHs), Electrophiles, and Oxidative Stress: Implications for the Alternative Pathway of PAH Activation Catalyzed by Human Dihydrodiol Dehydrogenase. Cancer Res. 1999; 59:607-614. [PubMed: 9973208]

712. Rao PSS, Kumar S. Polycyclic Aromatic Hydrocarbons and Cytochrome P450 in HIV Pathogenesis. Front Microbiol. 2015; 6:550. [PubMed: 26082767]

713. Nakajima M, Yoshida R, Shimada N, Yamazaki H, Yokoi T. Inhibition and Inactivation of Human Cytochrome P450 Isoforms by Phenethyl Isothiocyanate. Drug Metab Disposition. 2001; 29:1110-1113.

714. Grune T, Davies KJA. The Proteasomal System and HNE-Modified Proteins. Mol Aspects Med. 2003; 24:195-204. [PubMed: 12892997]

715. Ferrington DA, Kapphahn RJ. Catalytic Site-Specific Inhibition of the 20S Proteasome by 4Hydroxynonenal. FEBS Lett. 2004; 578:217-223. [PubMed: 15589823]

716. Shringarpure R, Grune T, Sitte N, Davies KJA. 4-Hydroxynonenal-Modified Amyloid- $\beta$ Peptide Inhibits the Proteasome: Possible Importance in Alzheimer's Disease. Cell Mol Life Sci. 2000; 57:1802-1809. [PubMed: 11130184]

717. Liu Y, Xu G, Sayre LM. Carnosine Inhibits (E)-4-Hydroxy-2-nonenal-Induced Protein CrossLinking: Structural Characterization of Carnosine-HNE Adducts. Chem Res Toxicol. 2003; 16:1589-1597. [PubMed: 14680373]

718. Zarkovic K. 4-Hydroxynonenal and Neurodegenerative Diseases. Mol Aspects Med. 2003; 24:293-303. [PubMed: 12893007]

719. Bosch-Morell F, Flohé L, Marín N, Romero FJ. 4-Hydroxynonenal Inhibits Glutathione Peroxidase: Protection by Glutathione. Free Radical Biol Med. 1999; 26:1383-1387. [PubMed: 10401601]

720. Zhao Y, Long MJC, Wang Y, Zhang S, Aye Y. Ube2V2 is a Rosetta Stone Bridging Redox and Ubiquitin Codes, Coordinating DNA Damage Responses. ACS Cent Sci. 2018; 4:246-259. [PubMed: 29532025]

721. Grimsrud PA, Picklo MJ, Griffin TJ, Bernlohr DA. Carbonylation of Adipose Proteins in Obesity and Insulin Resistance: Identification of Adipocyte Fatty Acid-Binding Protein as a Cellular Target of 4-Hydroxynonenal. Mol Cell Proteomics. 2007; 6:624-637. [PubMed: 17205980] 
722. Hellberg K, Grimsrud PA, Kruse AC, Banaszak LJ, Ohlendorf DH, Bernlohr DA. X-ray Crystallographic Analysis of Adipocyte Fatty Acid Binding Protein (aP2) Modified with 4Hydroxy-2-nonenal. Protein Sci. 2010; 19:1480-1489. [PubMed: 20509169]

723. Dwivedi S, Sharma A, Patrick B, Sharma R, Awasthi YC. Role of 4-Hydroxynonenal and its Metabolites in Signaling. Redox Rep. 2007; 12:4-10. [PubMed: 17263900]

724. Uchida K, Shiraishi M, Naito Y, Torii Y, Nakamura Y, Osawa T. Activation of Stress Signaling Pathways by the End Product of Lipid Peroxidation: 4-Hydroxy-2-nonenal is a Potential Inducer of Intracellular Peroxide Production. J Biol Chem. 1999; 274:2234-2242. [PubMed: 9890986]

725. Awasthi YC, Sharma R, Cheng JZ, Yang Y, Sharma A, Singhal SS, Awasthi S. Role of 4Hydroxynonenal in Stress-Mediated Apoptosis Signaling. Mol Aspects Med. 2003; 24:219-230. [PubMed: 12893000]

726. Murphy MP, Echtay KS, Blaikie FH, Asin-Cayuela J, Cochemé HM, Green K, Buckingham JA, Taylor ER, Hurrell F, Hughes G, et al. Superoxide Activates Uncoupling Proteins by Generating Carbon-Centered Radicals and Initiating Lipid Peroxidation: Studies Using a MitochondriaTargeted Spin Trap Derived from a-Phenyl-n-tert-butylnitrone. J Biol Chem. 2003; 278:4853448545. [PubMed: 12972420]

727. Chen ZH, Saito Y, Yoshida Y, Sekine A, Noguchi N, Niki E. 4-Hydroxynonenal Induces Adaptive Response and Enhances PC12 Cell Tolerance Primarily Through Induction of Thioredoxin Reductase 1 via Activation of Nrf2. J Biol Chem. 2005; 280:41921-41927. [PubMed: 16219762]

728. Chen ZH, Yoshida Y, Saito Y, Noguchi N, Niki E. Adaptive Response Induced by Lipid Peroxidation Products in Cell Cultures. FEBS Lett. 2006; 580:479-483. [PubMed: 16386737]

729. Davies S, Pandian R. Carfilzomib Proteasome Inhibitor, Oncolytic. Drugs Future. 2009; 34:708.

730. Schwartz PA, Kuzmic P, Solowiej J, Bergqvist S, Bolanos B, Almaden C, Nagata A, Ryan K, Feng J, Dalvie D, et al. Covalent EGFR Inhibitor Analysis Reveals Importance of Reversible Interactions to Potency and Mechanisms of Drug Resistance. Proc Natl Acad Sci U S A. 2014; 111:173-178. [PubMed: 24347635]

731. Aye Y, Li M, Long MJC, Weiss RS. Ribonucleotide Reductase and Cancer: Biological Mechanisms and Targeted Therapies. Oncogene. 2014; 34:2011. [PubMed: 24909171]

732. Stubbe J, Van Der Donk WA. Ribonucleotide Reductases: Radical Enzymes with Suicidal Tendencies. Chem Biol. 1995; 2:793-801. [PubMed: 8807812]

733. Albeck A, Kliper S. Mechanism of Cysteine Protease Inactivation by Peptidyl Epoxides. Biochem J. 1997; 322:879-884. [PubMed: 9148764]

734. Tong GC, Cornwell WK, Means GE. Reactions of Acrylamide with Glutathione and Serum Albumin. Toxicol Lett. 2004; 147:127-131. [PubMed: 14757316]

735. Lowther WT, Mcmillen DA, Orville AM, Matthews BW. The Anti-Angiogenic Agent Fumagillin Covalently Modifies a Conserved Active-Site Histidine in the Escherichia coli Methionine Aminopeptidase. Proc Natl Acad Sci U S A. 1998; 95:12153-12157. [PubMed: 9770455]

736. Joharapurkar AA, Dhanesha NA, Jain MR. Inhibition of the Methionine Aminopeptidase 2 Enzyme for the Treatment of Obesity. Diabetes Metab Syndr Obes. 2014; 7:73-84. [PubMed: 24611021]

737. Powers JC, Asgian JL, Ekici OD, James KE. Irreversible Inhibitors of Serine, Cysteine, and Threonine Proteases. Chem Rev. 2002; 102:4639-4750. [PubMed: 12475205]

738. Hedstrom L. Serine Protease Mechanism and Specificity. Chem Rev. 2002; 102:4501-4523. [PubMed: 12475199]

739. Whyte GF, Vilar R, Woscholski R. Molecular Recognition with Boronic Acids-Applications in Chemical Biology. J Chem Biol. 2013; 6:161-174. [PubMed: 24432132]

740. Kisselev Alexei fVan der linden WA, Overkleeft Herman s. Proteasome Inhibitors: An Expanding Army Attacking a Unique Target. Chem Biol. 2012; 19:99-115. [PubMed: 22284358]

741. Zaro BW, Whitby LR, Lum KM, Cravatt BF. Metabolically Labile Fumarate Esters Impart Kinetic Selectivity to Irreversible Inhibitors. J Am Chem Soc. 2016; 138:15841-15844. [PubMed: 27960302]

742. Singh MN, Hemant KSY, Ram M, Shivakumar HG. Microencapsulation: A Promising Technique for Controlled Drug Delivery. Res Pharm Sci. 2010; 5:65-77. [PubMed: 21589795] 
743. De Jong WH, Borm PJA. Drug Delivery and Nanoparticles: Applications and Hazards. Int J Nanomed. 2008; 3:133-149.

744. Firer MA, Gellerman G. Targeted Drug Delivery for Cancer Therapy: The Other Side of Antibodies. J Hematol Oncol. 2012; 5:70-70. [PubMed: 23140144]

745. Xie L, Xie L, Kinnings SL, Bourne PE. Novel Computational Approaches to Polypharmacology as a Means to Define Responses to Individual Drugs. Annu Rev Pharmacol Toxicol. 2012; 52:361-379. [PubMed: 22017683]

746. Wang X, Thijssen B, Yu H. Target Essentiality and Centrality Characterize Drug Side Effects. PLoS Comp Biol. 2013; 9:e1003119.

747. Woolley JF, Stanicka J, Cotter TG. Recent Advances in Reactive Oxygen Species Measurement in Biological Systems. Trends Biochem Sci. 2013; 38:556-565. [PubMed: 24120034]

748. Kalyanaraman B, Darley-Usmar V, Davies KJA, Dennery PA, Forman HJ, Grisham MB, Mann GE, Moore K, Roberts LJ, Ischiropoulos H. Measuring Reactive Oxygen and Nitrogen Species with Fluorescent Probes: Challenges and Limitations. Free Radical Biol Med. 2012; 52:1-6. [PubMed: 22027063]

749. Lin VS, Dickinson BC, Chang CJ. Boronate-Based Fluorescent Probes: Imaging Hydrogen Peroxide in Living Systems. Methods Enzymol. 2013; 526:19-43. [PubMed: 23791092]

750. Guo H, Aleyasin H, Dickinson BC, Haskew-Layton RE, Ratan RR. Recent Advances in Hydrogen Peroxide Imaging for Biological Applications. Cell Biosci. 2014; 4:64. [PubMed: 25400906]

751. Chen X, Zhong Z, Xu Z, Chen L, Wang Y. 2', $7^{\prime}$-Dichlorodihydrofluorescein as a Fluorescent Probe for Reactive Oxygen Species Measurement: Forty Years of Application and Controversy. Free Radic Res. 2010; 44:587-604. [PubMed: 20370560]

752. Wardman P. Fluorescent and Luminescent Probes for Measurement of Oxidative and Nitrosative Species in Cells and Tissues: Progress, Pitfalls, and Prospects. Free Radical Biol Med. 2007; 43:995-1022. [PubMed: 17761297]

753. Fletcher MP, Halpern GM. Effects of Low Concentrations of Arachidonic Acid Derived Mediators on the Membrane Potential and Respiratory Burst Responses of Human Neutrophils as Assessed by Flow Cytometry. Fundam Clin Pharmacol. 1990; 4:65-77. [PubMed: 2160416]

754. Rothe G, Valet G. Flow Cytometric Analysis of Respiratory Burst Activity in Phagocytes with Hydroethidine and 2',7' -Dichlorofluorescin. J Leukoc Biol. 1990; 47:440-448. [PubMed: 2159514]

755. Wan CP, Myung E, Lau BHS. An Automated Micro-Fluorometric Assay for Monitoring Oxidative Burst Activity of Phagocytes. J Immunol Methods. 1993; 159:131-138. [PubMed: 8445246]

756. Lebel CP, Ischiropoulos H, Bondy SC. Evaluation of the Probe $2^{\prime}, 7^{\prime}$-Dichlorofluorescin as an Indicator of Reactive Oxygen Species Formation and Oxidative Stress. Chem Res Toxicol. 1992; 5:227-231. [PubMed: 1322737]

757. Wrona M, Patel K, Wardman P. Reactivity of $2^{\prime}, 7^{\prime}$-Dichlorodihydrofluorescein and Dihydrorhodamine 123 and their Oxidized Forms Toward Carbonate, Nitrogen Dioxide, and Hydroxyl Radicals. Free Radical Biol Med. 2005; 38:262-270. [PubMed: 15607909]

758. Zhu H, Bannenberg GL, Moldéus P, Shertzer HG. Oxidation Pathways for the Intracellular Probe $2^{\prime}, 7^{\prime}$-Dichlorofluorescin. Arch Toxicol. 1994; 68:582-587. [PubMed: 7998826]

759. Brömme HJ, Zühlke L, Silber RE, Simm A. DCFH2 Interactions with Hydroxyl Radicals and Other Oxidants - Influence of Organic Solvents. Exp Gerontol. 2008; 43:638-644. [PubMed: 18337037]

760. Daghastanli NA, Itri R, Baptista MS. Singlet Oxygen Reacts with $2^{\prime}, 7^{\prime}$ Dichlorodihydrofluorescein and Contributes to the Formation of $2^{\prime}, 7^{\prime}$-Dichlorofluorescein. Photochem Photobiol. 2008; 84:1238-1243. [PubMed: 18422880]

761. Possel H, Noack H, Augustin W, Keilhoff G, Wolf G. 2,7-Dihydrodichlorofluorescein Diacetate as a Fluorescent Marker for Peroxynitrite Formation. FEBS Lett. 1997; 416:175-178. [PubMed: 9369208] 
762. Peng T, Wong NK, Chen X, Chan YK, Ho DHH, Sun Z, Hu JJ, Shen J, El-Nezami H, Yang D. Molecular Imaging of Peroxynitrite with HKgreen-4 in Live Cells and Tissues. J Am Chem Soc. 2014; 136:11728-11734. [PubMed: 25058034]

763. Yang D, Wang HL, Sun ZN, Chung NW, Shen JG. A Highly Selective Fluorescent Probe for the Detection and Imaging of Peroxynitrite in Living Cells. J Am Chem Soc. 2006; 128:6004-6005. [PubMed: 16669647]

764. Carballal S, Bartesaghi S, Radi R. Kinetic and Mechanistic Considerations to Assess the Biological Fate of Peroxynitrite. Biochim Biophys Acta. 2014; 1840:768-780. [PubMed: 23872352]

765. Setsukinai KI, Urano Y, Kakinuma K, Majima HJ, Nagano T. Development of Novel Fluorescence Probes that can Reliably Detect Reactive Oxygen Species and Distinguish Specific Species. J Biol Chem. 2003; 278:3170-3175. [PubMed: 12419811]

766. Koide Y, Urano Y, Kenmoku S, Kojima H, Nagano T. Design and Synthesis of Fluorescent Probes for Selective Detection of Highly Reactive Oxygen Species in Mitochondria of Living Cells. J Am Chem Soc. 2007; 129:10324-10325. [PubMed: 17672465]

767. Royall JA, Ischiropoulos H. Evaluation of $2^{\prime}, 7^{\prime}$-Dichlorofluorescin and Dihydrorhodamine as Fluorescent Probes for Intracellular H2o2 in Cultured Endothelial Cells. Arch Biochem Biophys. 1993; 302:348-355. [PubMed: 8387741]

768. Crow JP. Dichlorodihydrofluorescein and Dihydrorhodamine 123 are Sensitive Indicators of Peroxynitritein in Vitro: Implications for Intracellular Measurement of Reactive Nitrogen and Oxygen Species. Nitric Oxide. 1997; 1:145-157. [PubMed: 9701053]

769. Zielonka J, Sarna T, Roberts JE, Wishart JF, Kalyanaraman B. Pulse Radiolysis and Steady-State Analyses of the Reaction Between Hydroethidine and Superoxide and Other Oxidants. Arch Biochem Biophys. 2006; 456:39-47. [PubMed: 17081495]

770. Zielonka J, Kalyanaraman B. Hydroethidine- and Mito-SOX-Derived Red Fluorescence is not a Reliable Indicator of Intracellular Superoxide Formation: Another Inconvenient Truth. Free Radical Biol Med. 2010; 48:983-1001. [PubMed: 20116425]

771. Zielonka J, Vasquez-Vivar J, Kalyanaraman B. Detection of 2-Hydroxyethidium in Cellular Systems: A Unique Marker Product of Superoxide and Hydroethidine. Nat Protocols. 2008; 3:821. [PubMed: 18193017]

772. Robinson KM, Janes MS, Beckman JS. The Selective Detection of Mitochondrial Superoxide by Live Cell Imaging. Nat Protoc. 2008; 3:941-947. [PubMed: 18536642]

773. Mukhopadhyay P, Rajesh M, Yoshihiro K, Haskó G, Pacher P. Simple Quantitative Detection of Mitochondrial Superoxide Production in Live Cells. Biochem Biophys Res Commun. 2007; 358:203-208. [PubMed: 17475217]

774. Sensi SL, Yin HZ, Carriedo SG, Rao SS, Weiss JH. Preferential Zn(2+) Influx Through Ca(2+)Permeable AMPA/Kainate Channels Triggers Prolonged Mitochondrial Superoxide Production. Proc Natl Acad Sci U S A. 1999; 96:2414-2419. [PubMed: 10051656]

775. Quintana-Cabrera R, Fernandez-Fernandez S, Bobo-Jimenez V, Escobar J, Sastre J, Almeida A, Bolaños JP. $\gamma$-Glutamylcysteine Detoxifies Reactive Oxygen Species by Acting as Glutathione Peroxidase-1 Cofactor. Nat Commun. 2012; 3:718. [PubMed: 22395609]

776. Dębski D, Smulik R, Zielonka J, Michałowski B, Jakubowska M, Dębowska K, Adamus J, Marcinek A, Kalyanaraman B, Sikora A. Mechanism of Oxidative Conversion of Amplex® Red to Resorufin: Pulse Radiolysis and Enzymatic Studies. Free Radical Biol Med. 2016; 95:323332. [PubMed: 27021961]

777. Zhou M, Diwu Z, Panchuk-Voloshina N, Haugland RP. A Stable Nonfluorescent Derivative of Resorufin for the Fluorometric Determination of Trace Hydrogen Peroxide: Applications in Detecting the Activity of Phagocyte NADPH Oxidase and Other Oxidases. Anal Biochem. 1997; 253:162-168. [PubMed: 9367498]

778. Towne V, Will M, Oswald B, Zhao Q. Complexities in Horseradish Peroxidase-Catalyzed Oxidation of Dihydroxyphenoxazine Derivatives: Appropriate Ranges for $\mathrm{pH}$ Values and Hydrogen Peroxide Concentrations in Quantitative Analysis. Anal Biochem. 2004; 334:290-296. [PubMed: 15494136] 
779. Votyakova TV, Reynolds IJ. Detection of Hydrogen Peroxide with Amplex Red: Interference by NADH and Reduced Glutathione Auto-Oxidation. Arch Biochem Biophys. 2004; 431:138-144. [PubMed: 15464736]

780. Kettle AJ, Carr AC, Winterbourn CC. Assays Using Horseradish Peroxidase and Phenolic Substrates Require Superoxide Dismutase for Accurate Determination of Hydrogen Peroxide Production by Neutrophils. Free Radical Biol Med. 1994; 17:161-164. [PubMed: 7959174]

781. O'brien J, Wilson I, Orton T, Pognan F. Investiagtion of the Alamar Blue (Resazurin) Fluorescent Dye for the Assessment of Mammalian Cell Cytotoxicity. Eur J Biochem. 2000; 267:5421-5426. [PubMed: 10951200]

782. Panizzi P, Nahrendorf M, Wildgruber M, Waterman P, Figueiredo JL, Aikawa E, Mccarthy J, Weissleder R, Hilderbrand SA. Oxazine Conjugated Nanoparticle Detects in Vivo Hypochlorous Acid and Peroxynitrite Generation. J Am Chem Soc. 2009; 131:15739-15744. [PubMed: 19817443]

783. Koide Y, Urano Y, Hanaoka K, Terai T, Nagano T. Development of an Si-Rhodamine-Based FarRed to Near-Infrared Fluorescence Probe Selective for Hypochlorous Acid and Its Applications for Biological Imaging. J Am Chem Soc. 2011; 133:5680-5682. [PubMed: 21443186]

784. Liu SR, Wu SP. Hypochlorous Acid Turn-On Fluorescent Probe Based on Oxidation of Diphenyl Selenide. Org Lett. 2013; 15:878-881. [PubMed: 23373559]

785. Yuan L, Wang L, Agrawalla BK, Park SJ, Zhu H, Sivaraman B, Peng J, Xu QH, Chang YT. Development of Targetable Two-Photon Fluorescent Probes to Image Hypochlorous Acid in Mitochondria and Lysosome in Live Cell and Inflamed Mouse Model. J Am Chem Soc. 2015; 137:5930-5938. [PubMed: 25905448]

786. Miller EW, Bian SX, Chang CJ. A Fluorescent Sensor for Imaging Reversible Redox Cycles in Living Cells. J Am Chem Soc. 2007; 129:3458-3459. [PubMed: 17335279]

787. Lippert AR, Van De Bittner GC, Chang CJ. Boronate Oxidation as a Bioorthogonal Reaction Approach for Studying the Chemistry of Hydrogen Peroxide in Living Systems. Acc Chem Res. 2011; 44:793-804. [PubMed: 21834525]

788. Chang MCY, Pralle A, Isacoff EY, Chang CJ. A Selective, Cell-Permeable Optical Probe for Hydrogen Peroxide in Living Cells. J Am Chem Soc. 2004; 126:15392-15393. [PubMed: 15563161]

789. Sikora A, Zielonka J, Lopez M, Joseph J, Kalyanaraman B. Direct Oxidation of Boronates by Peroxynitrite: Mechanism and Implications in Fluorescence Imaging of Peroxynitrite. Free Radical Biol Med. 2009; 47:1401-1407. [PubMed: 19686842]

790. Miller EW, Tulyathan O, Isacoff EY, Chang CJ. Molecular Imaging of Hydrogen Peroxide Produced for Cell Signaling. Nat Chem Biol. 2007; 3:263-267. [PubMed: 17401379]

791. Dickinson BC, Huynh C, Chang CJ. A Palette of Fluorescent Probes with Varying Emission Colors for Imaging Hydrogen Peroxide Signaling in Living Cells. J Am Chem Soc. 2010; 132:5906-5915. [PubMed: 20361787]

792. Albers AE, Okreglak VS, Chang CJ. A FRET-Based Approach to Ratiometric Fluorescence Detection of Hydrogen Peroxide. J Am Chem Soc. 2006; 128:9640-9641. [PubMed: 16866512]

793. Srikun D, Miller EW, Domaille DW, Chang CJ. An ICT-Based Approach to Ratiometric Fluorescence Imaging of Hydrogen Peroxide Produced in Living Cells. J Am Chem Soc. 2008; 130:4596-4597. [PubMed: 18336027]

794. Dickinson BC, Tang Y, Chang Z, Chang CJ. Development of a Nuclear-Localized Fluorescent Probe for Hydrogen Peroxide and Applications to the Study of Sirtuin-Mediated Oxidative Stress Responses in Vivo. Chem Biol. 2011; 18:943-948. [PubMed: 21867909]

795. Brown HC, Zweifel G. A Stereospecific cis Hydration of the Double Bond in Cyclic Derivatives. J Am Chem Soc. 1959; 81:247-247.

796. Brewer TF, Garcia FJ, Onak CS, Carroll KS, Chang CJ. Chemical Approaches to Discovery and Study of Sources and Targets of Hydrogen Peroxide Redox Signaling Through NADPH Oxidase Proteins. Annu Rev Biochem. 2015; 84:765-790. [PubMed: 26034893]

797. Dickinson BC, Peltier J, Stone D, Schaffer DV, Chang CJ. Nox2 Redox Signaling Maintains Essential Cell Populations in the Brain. Nat Chem Biol. 2011; 7:106-112. [PubMed: 21186346] 
798. Elian M, Chen MML, Mingos DMP, Hoffmann R. Comparative Bonding Study of Conical Fragments. Inorg Chem. 1976; 15:1148-1155.

799. Dickinson BC, Huynh C, Chang CJ. A palette of fluorescent probes with varying emission colors for imaging hydrogen peroxide signaling in living cells. J Am Chem Soc. 2010:132.

800. Dickinson BC, Chang CJ. A Targetable Fluorescent Probe for Imaging Hydrogen Peroxide in the Mitochondria of Living Cells. J Am Chem Soc. 2008; 130:9638-9639. [PubMed: 18605728]

801. Masanta G, Heo CH, Lim CS, Bae SK, Cho BR, Kim HM. A Mitochondria-Localized TwoPhoton Fluorescent Probe for Ratiometric Imaging of Hydrogen Peroxide in Live Tissue. Chem Commun. 2012; 48:3518-3520.

802. Cocheme HM, Logan A, Prime TA, Abakumova I, Quin C, Mcquaker SJ, Patel JV, Fearnley IM, James AM, Porteous CM, et al. Using the Mitochondria-Targeted Ratiometric Mass Spectrometry Probe MitoB to Measure H2O2 in Living Drosophila. Nat Protoc. 2012; 7:946958. [PubMed: 22517261]

803. Murphy MP, Smith RaJ. Targeting Antioxidants to Mitochondria by Conjugation to Lipophilic Cations. Annu Rev Pharmacol Toxicol. 2007; 47:629-656. [PubMed: 17014364]

804. Srikun D, Albers AE, Nam CI, Iavarone AT, Chang CJ. Organelle-Targetable Fluorescent Probes for Imaging Hydrogen Peroxide in Living Cells via SNAP-Tag Protein Labeling. J Am Chem Soc. 2010; 132:4455-4465. [PubMed: 20201528]

805. Østergaard H, Henriksen A, Hansen FG, Winther JR. Shedding Light on Disulfide Bond Formation: Engineering a Redox Switch in Green Fluorescent Protein. EMBO J. 2001; 20:58535862. [PubMed: 11689426]

806. Wang W, Fang H, Groom L, Cheng A, Zhang W, Liu J, Wang X, Li K, Han P, Zheng M, et al. Superoxide Flashes in Single Mitochondria. Cell. 2008; 134:279-290. [PubMed: 18662543]

807. Schwarzländer M, Murphy MP, Duchen MR, Logan DC, Fricker MD, Halestrap AP, Müller FL, Rizzuto R, Dick TP, Meyer AJ, et al. Mitochondrial Flashes: A Radical Concept RepHined. Trends Cell Biol. 2012; 22:503-508. [PubMed: 22917552]

808. Poburko D, Santo-Domingo J, Demaurex N. Dynamic Regulation of the Mitochondrial Proton Gradient During Cytosolic Calcium Elevations. J Biol Chem. 2011; 286:11672-11684. [PubMed: 21224385]

809. Schwarzlander M, Wagner S, Ermakova YG, Belousov VV, Radi R, Beckman JS, Buettner GR, Demaurex N, Duchen MR, Forman HJ, et al. The Mitoflash Probe cpYFP Does Not Respond to Superoxide. Nature. 2014; 514:E12-E14. [PubMed: 25341790]

810. Lukyanov KA, Belousov VV. Genetically Encoded Fluorescent Redox Sensors. Biochim Biophys Acta. 2014; 1840:745-756. [PubMed: 23726987]

811. Gutscher M, Pauleau AL, Marty L, Brach T, Wabnitz GH, Samstag Y, Meyer AJ, Dick TP. RealTime Imaging of the Intracellular Glutathione Redox Potential. Nat Methods. 2008; 5:553-559. [PubMed: 18469822]

812. Meyer AJ, Dick TP. Fluorescent Protein-Based Redox Probes. Antioxid Redox Signal. 2010; 13:621-650. [PubMed: 20088706]

813. Hanson GT, Aggeler R, Oglesbee D, Cannon M, Capaldi RA, Tsien RY, Remington SJ. Investigating Mitochondrial Redox Potential with Redox-Sensitive Green Fluorescent Protein Indicators. J Biol Chem. 2004; 279:13044-13053. [PubMed: 14722062]

814. Gutscher M, Sobotta MC, Wabnitz GH, Ballikaya S, Meyer AJ, Samstag Y, Dick TP. ProximityBased Protein Thiol Oxidation by H2O2-Scavenging Peroxidases. J Biol Chem. 2009; 284:31532-31540. [PubMed: 19755417]

815. Morgan B, Van Laer K, Owusu TNE, Ezerina D, Pastor-Flores D, Amponsah PS, Tursch A, Dick TP. Real-Time Monitoring of Basal H2O2 Levels with Peroxiredoxin-Based Probes. Nat Chem Biol. 2016; 12:437-443. [PubMed: 27089028]

816. Belousov VV, Fradkov AF, Lukyanov KA, Staroverov DB, Shakhbazov KS, Terskikh AV, Lukyanov S. Genetically Encoded Fluorescent Indicator for Intracellular Hydrogen Peroxide. Nat Methods. 2006; 3:281-286. [PubMed: 16554833]

817. Bilan DS, Belousov VV. HyPer Family Probes: State of the Art. Antioxid Redox Signal. 2015; 24:731-751. 
818. Belousov VV, Fradkov AF, Lukyanov KA, Staroverov DB, Shakhbazov KS, Terskikh AV, Lukyanov S. Genetically encoded fluorescent indicator for intracellular hydrogen peroxide. Nat Methods. 2006:3.

819. Markvicheva KN, Bilan DS, Mishina NM, Gorokhovatsky AY, Vinokurov LM, Lukyanov S, Belousov VV. A Genetically Encoded Sensor for H2O2 with Expanded Dynamic Range. Biorg Med Chem. 2011; 19:1079-1084.

820. Bilan DS, Pase L, Joosen L, Gorokhovatsky AY, Ermakova YG, Gadella TWJ, Grabher C, Schultz C, Lukyanov S, Belousov VV. HyPer-3: A Genetically Encoded H2O2 Probe with Improved Performance for Ratiometric and Fluorescence Lifetime Imaging. ACS Chem Biol. 2013; 8:535542. [PubMed: 23256573]

821. Morinaka A, Yamada M, Itofusa R, Funato Y, Yoshimura Y, Nakamura F, Yoshimura T, Kaibuchi K, Goshima Y, Hoshino M, et al. Thioredoxin Mediates Oxidation-Dependent Phosphorylation of CRMP2 and Growth Cone Collapse. Sci Signaling. 2011; 4:ra26-ra26.

822. Miller EW, Dickinson BC, Chang CJ. Aquaporin-3 Mediates Hydrogen Peroxide Uptake to Regulate Downstream Intracellular Signaling. Proc Natl Acad Sci U S A. 2010; 107:1568115686. [PubMed: 20724658]

823. Love NR, Chen Y, Ishibashi S, Kritsiligkou P, Lea R, Koh Y, Gallop JL, Dorey K, Amaya E. Amputation-Induced Reactive Oxygen Species are Required for Successful Xenopus Tadpole Tail Regeneration. Nat Cell Biol. 2013; 15:222. [PubMed: 23314862]

824. Niethammer P, Grabher C, Look AT, Mitchison TJ. A tissue-scale gradient of hydrogen peroxide mediates rapid wound detection in zebrafish. Nature. 2009:459.

825. Ermakova YG, Bilan DS, Matlashov ME, Mishina NM, Markvicheva KN, Subach OM, Subach FV, Bogeski I, Hoth M, Enikolopov G, et al. Red Fluorescent Genetically Encoded Indicator for Intracellular Hydrogen Peroxide. Nat Commun. 2014; 5:5222. [PubMed: 25330925]

826. Irina M, Mathilde Van-Melle G, Bernard C, Corinne P, Jean-Luc R. Green Autofluorescence, a Double Edged Monitoring Tool for Bacterial Growth and Activity in Micro-Plates. Phys Biol. 2015; 12:066016. [PubMed: 26656747]

827. Croce AC, Bottiroli G. Autofluorescence Spectroscopy and Imaging: A Tool for Biomedical Research and Diagnosis. Eur J Histochem. 2014; 58:2461. [PubMed: 25578980]

828. Simen Zhao B, Liang Y, Song Y, Zheng C, Hao Z, Chen PR. A Highly Selective Fluorescent Probe for Visualization of Organic Hydroperoxides in Living Cells. J Am Chem Soc. 2010; 132:17065-17067. [PubMed: 21077671]

829. Ermakova YG, Bilan DS, Matlashov ME, Mishina NM, Markvicheva KN, Subach OM, Subach FV, Bogeski I, Hoth M, Enikolopov G, et al. Red fluorescent genetically encoded indicator for intracellular hydrogen peroxide. 2014; 5:5222.

830. Zhao BS, Zhang G, Zeng S, He C, Chen PR. Probing Subcellular Organic Hydroperoxide Formation via a Genetically Encoded Ratiometric and Reversible Fluorescent Indicator. Integr Biol. 2013; 5:1485-1489.

831. Spasojević I. Free Radicals and Antioxidants at a Glance Using EPR Spectroscopy. Crit Rev Clin Lab Sci. 2011; 48:114-142. [PubMed: 21875311]

832. Villamena FA, Zweier JL. Detection of Reactive Oxygen and Nitrogen Species by EPR Spin Trapping. Antioxid Redox Signal. 2004; 6:619-629. [PubMed: 15130289]

833. Roubaud V, Sankarapandi S, Kuppusamy P, Tordo P, Zweier JL. Quantitative Measurement of Superoxide Generation Using The Spin Trap 5-(diethoxyphosphoryl)-5-methyl- 1-pyrroline-noxide. Anal Biochem. 1997; 247:404-411. [PubMed: 9177705]

834. Gopalakrishnan B, Nash KM, Velayutham M, Villamena FA. Detection of Nitric Oxide and Superoxide Radical Anion by Electron Paramagnetic Resonance Spectroscopy from Cells Using Spin Traps. J Vis Exp. 2012; doi: 10.3791/28102810

835. Abbas K, Babić N, Peyrot F. Use Of Spin Traps To Detect Superoxide Production In Living Cells By Electron Paramagnetic Resonance (EPR) Spectroscopy. Methods. 2016; 109:31-43. [PubMed: 27163864]

836. Villamena FA, Zweier JL. Detection of Reactive Oxygen and Nitrogen Species by EPR Spin Trapping. Antioxidants \& redox signaling. 2004; 6:619-629. [PubMed: 15130289] 
837. Kelicen P, Cantuti-Castelvetri I, Pekiner C, Paulson KE. The Spin Trapping Agent PBN Stimulates H2O2-Induced Erk and Src Kinase Activity in Human Neuroblastoma Cells. NeuroReport. 2002; 13:1057-1061. [PubMed: 12060808]

838. Das A, Gopalakrishnan B, Voss OH, Doseff AI, Villamena FA. Inhibition of ROS-Induced Apoptosis in Endothelial Cells by Nitrone Spin Traps via Induction of Phase Ii Enzymes and Suppression of Mitochondria-Dependent Pro-Apoptotic Signaling. Biochem Pharmacol. 2012; 84:486-497. [PubMed: 22580046]

839. Hardy M, Poulhés F, Rizzato E, Rockenbauer A, Banaszak K, Karoui H, Lopez M, Zielonka J, Vasquez-Vivar J, Sethumadhavan S, et al. Mitochondria-Targeted Spin Traps: Synthesis, Superoxide Spin Trapping, and Mitochondrial Uptake. Chem Res Toxicol. 2014; 27:1155-1165. [PubMed: 24890552]

840. Han Y, Liu Y, Rockenbauer A, Zweier JL, Durana G, Villamena FA. Lipophilic $\beta$-Cyclodextrin Cyclic-Nitrone Conjugate: Synthesis and Spin Trapping Studies. J Org Chem. 2009; 74:53695380. [PubMed: 19530689]

841. Han Y, Tuccio B, Lauricella R, Villamena FA. Improved Spin Trapping Properties by $\beta$ Cyclodextrin-Cyclic Nitrone Conjugate. J Org Chem. 2008; 73:7108-7117. [PubMed: 18707169]

842. Abbas K, Hardy M, Poulhès F, Karoui H, Tordo P, Ouari O, Peyrot F. Detection of Superoxide Production in Stimulated and Unstimulated Living Cells Using New Cyclic Nitrone Spin Traps. Free Radical Biol Med. 2014; 71:281-290. [PubMed: 24662195]

843. Bézière N, Hardy M, Poulhès F, Karoui H, Tordo P, Ouari O, Frapart Y-M, Rockenbauer A, Boucher J-L, Mansuy D, et al. Metabolic Stability of Superoxide Adducts Derived from Newly Developed Cyclic Nitrone Spin Traps. Free Radical Biol Med. 2014; 67:150-158. [PubMed: 24161442]

844. Hissung A, Isildar M, Von Sonntag C, Witzel H. Radiolysis of Aqueous Solutions of Nucleosides Halogenated at the Sugar Moiety. Int J Radiat Biol Relat Stud Phys, Chem Med. 1981; 39:185193. [PubMed: 6453102]

845. Venkataraman S, Schafer FQ, Buettner GR. Detection of Lipid Radicals Using EPR. Antioxid Redox Signal. 2004; 6:631-638. [PubMed: 15130290]

846. North JA, Spector AA, Buettner GR. Detection of Lipid Radicals by Electron Paramagnetic Resonance Spin Trapping Using Intact Cells Enriched with Polyunsaturated Fatty Acid. J Biol Chem. 1992; 267:5743-5746. [PubMed: 1313417]

847. Stolze K, Udilova N, Nohl H. Spin Adducts of Superoxide, Alkoxyl, and Lipid-Derived Radicals with EMPO and its Derivatives. Biol Chem. 2002; 383:813-820. [PubMed: 12108546]

848. Marchand V, Charlier N, Verrax J, Buc-Calderon P, Levêque P, Gallez B. Use of a Cocktail of Spin Traps for Fingerprinting Large Range of Free Radicals in Biological Systems. PLoS One. 2017; 12:e0172998. [PubMed: 28253308]

849. Ranguelova K, Mason RP. The Fidelity of Spin Trapping with DMPO in Biological Systems. Magn Reson Chem. 2011; 49:152-158. [PubMed: 21246623]

850. Takayanagi T, Kimiya H, Ohyama T. Formation of Artifactual DMPO-OH Spin Adduct in Acid Solutions Containing Nitrite Ions. Free Radic Res. 2017; 51:739-748. [PubMed: 28817986]

851. Leinisch F, Jiang J, Derose EF, Khramtsov VV, Mason RP. Investigation of Spin-Trapping Artifacts Formed by the Forrester-Hepburn Mechanism. Free Radical Biol Med. 2013; 65:14971505. [PubMed: 23851031]

852. Swartz HM. Use of Nitroxides to Measure Redox Metabolism in Cells and Tissues. J Chem SOC Faraday Trans 1. 1987; 83:191-202.

853. Swartz HM, Khan N, Buckey J, Comi R, Gould L, Grinberg O, Hartford A, Hopf H, Hou H, Hug E, et al. Clinical Applications of EPR: Overview and Perspectives. NMR Biomed. 2004; 17:335351. [PubMed: 15366033]

854. Finkelstein E, Rosen GM, Rauckman EJ. Spin Trapping. Kinetics of the Reaction of Superoxide and Hydroxyl Radicals with Nitrones. J Am Chem Soc. 1980; 102:4994-4999.

855. Dikalov SI, Kirilyuk IA, Voinov M, Grigor'ev IA. EPR Detection of Cellular and Mitochondrial Superoxide Using Cyclic Hydroxylamines. Free Radic Res. 2011; 45:417-430. [PubMed: 21128732] 
856. Gomez-Mejiba SE, Zhai Z, Della-Vedova MC, Muñoz MD, Chatterjee S, Towner RA, Hensley K, Floyd RA, Mason RP, Ramirez DC. Immuno-Spin Trapping from Biochemistry to Medicine: Advances, Challenges, and Pitfalls. Focus on Protein-Centered Radicals. Biochim Biophys Acta. 2014; 1840:722-729. [PubMed: 23644035]

857. Heck HDA, White EL, Casanova-Schmitz M. Determination of Formaldehyde in Biological Tissues by Gas Chromatography/Mass Spectrometry. Biol Mass Spectrom. 1982; 9:347-353.

858. Tong Z, Han C, Luo W, Wang X, Li H, Luo H, Zhou J, Qi J, He R. Accumulated Hippocampal Formaldehyde Induces Age-Dependent Memory Decline. Age. 2013; 35:583-596. [PubMed: 22382760]

859. Brewer TF, Chang CJ. An Aza-Cope Reactivity-Based Fluorescent Probe for Imaging Formaldehyde in Living Cells. J Am Chem Soc. 2015; 137:10886-10889. [PubMed: 26306005]

860. Roth A, Li H, Anorma C, Chan J. A Reaction-Based Fluorescent Probe for Imaging of Formaldehyde in Living Cells. J Am Chem Soc. 2015; 137:10890-10893. [PubMed: 26305899]

861. Wang T, Douglass EF, Fitzgerald KJ, Spiegel DA. A “Turn-On” Fluorescent Sensor for Methylglyoxal. J Am Chem Soc. 2013; 135:12429-12433. [PubMed: 23931147]

862. Tang Y, Kong X, Xu A, Dong B, Lin W. Development of a Two-Photon Fluorescent Probe for Imaging of Endogenous Formaldehyde in Living Tissues. Angew Chem Int Ed. 2016; 55:33563359.

863. Bruemmer KJ, Brewer TF, Chang CJ. Fluorescent Probes for Imaging Formaldehyde in Biological Systems. Curr Opin Chem Biol. 2017; 39:17-23. [PubMed: 28527906]

864. Yuen LH, Saxena NS, Park HS, Weinberg K, Kool ET. Dark Hydrazone Fluorescence Labeling Agents Enable Imaging of Cellular Aldehydic Load. ACS Chem Biol. 2016; 11:2312-2319. [PubMed: 27326450]

865. Chen J, Zeng L, Xia T, Li S, Yan T, Wu S, Qiu G, Liu Z. Toward a Biomarker of Oxidative Stress: A Fluorescent Probe for Exogenous and Endogenous Malondialdehyde in Living Cells. Anal Chem. 2015; 87:8052-8056. [PubMed: 26200908]

866. Paulsen CE, Carroll KS. Orchestrating Redox Signaling Networks through Regulatory Cysteine Switches. ACS Chem Biol. 2010; 5:47-62. [PubMed: 19957967]

867. Salmeen A, Andersen JN, Myers MP, Meng TC, Hinks JA, Tonks NK, Barford D. Redox Regulation of Protein Tyrosine Phosphatase $1 \mathrm{~b}$ Involves a Sulphenyl-Amide Intermediate. Nature. 2003; 423:769-773. [PubMed: 12802338]

868. Wani R, Qian J, Yin L, Bechtold E, King SB, Poole LB, Paek E, Tsang AW, Furdui CM. IsoformSpecific Regulation of Akt by PDGF-Induced Reactive Oxygen Species. Proc Natl Acad Sci U S A. 2011; 108:10550-10555. [PubMed: 21670275]

869. Jaffrey SR, Erdjument-Bromage H, Ferris CD, Tempst P, Snyder SH. Protein S-Nitrosylation: A Physiological Signal for Neuronal Nitric Oxide. Nat Cell Biol. 2001; 3:193. [PubMed: 11175752]

870. Saurin AT, Neubert H, Brennan JP, Eaton P. Widespread Sulfenic Acid Formation in Tissues in Response to Hydrogen Peroxide. Proc Natl Acad Sci U S A. 2004; 101:17982-17987. [PubMed: 15604151]

871. García-Santamarina S, Boronat S, Domènech A, Ayté J, Molina H, Hidalgo E. Monitoring in Vivo Reversible Cysteine Oxidation in Proteins Using ICAT and Mass Spectrometry. Nat Protoc. 2014; 9:1131. [PubMed: 24743420]

872. Sethuraman M, Mccomb ME, Huang H, Huang S, Heibeck T, Costello CE, Cohen RA. IsotopeCoded Affinity Tag (ICAT) Approach to Redox Proteomics:w Identification and Quantitation of Oxidant-Sensitive Cysteine Thiols in Complex Protein Mixtures. J Proteome Res. 2004; 3:12281233. [PubMed: 15595732]

873. Sethuraman M, Clavreul N, Huang H, Mccomb ME, Costello CE, Cohen RA. Quantification of Oxidative Post-Translational Modifications of Cysteine Thiols of P21ras Associated with Redox Modulation of Activity Using Isotope-Coded Affinity Tags (ICAT) and Mass Spectrometry. Free Radical Biol Med. 2007; 42:823-829. [PubMed: 17320764]

874. Yang J, Carroll KS, Liebler DC. The Expanding Landscape of the Thiol Redox Proteome. Mol Cell Proteomics. 2016; 15:1-11. [PubMed: 26518762] 
875. Kumar V, Kleffmann T, Hampton MB, Cannell MB, Winterbourn CC. Redox Proteomics of Thiol Proteins in Mouse Heart During Ischemia/Reperfusion Using ICAT Reagents and Mass Spectrometry. Free Radical Biol Med. 2013; 58:109-117. [PubMed: 23376233]

876. Leichert LI, Gehrke F, Gudiseva HV, Blackwell T, Ilbert M, Walker AK, Strahler JR, Andrews PC, Jakob U. Quantifying Changes in the Thiol Redox Proteome upon Oxidative Stress in Vivo. Proc Natl Acad Sci U S A. 2008; 105:8197-8202. [PubMed: 18287020]

877. Go YM, Roede JR, Walker DI, Duong DM, Seyfried NT, Orr M, Liang Y, Pennell KD, Jones DP. Selective Targeting of the Cysteine Proteome by Thioredoxin and Glutathione Redox Systems. Mol Cell Proteomics. 2013; 12:3285-3296. [PubMed: 23946468]

878. Kato D, Boatright KM, Berger AB, Nazif T, Blum G, Ryan C, Chehade KaH, Salvesen GS, Bogyo M. Activity-Based Probes that Target Diverse Cysteine Protease Families. Nat Chem Biol. 2005; 1:33-38. [PubMed: 16407991]

879. Liu Y, Patricelli MP, Cravatt BF. Activity-Based Protein Profiling: The Serine Hydrolases. Proc Natl Acad Sci U S A. 1999; 96:14694-14699. [PubMed: 10611275]

880. Weerapana E, Simon GM, Cravatt BF. Disparate Proteome Reactivity Profiles of Carbon Electrophiles. Nat Chem Biol. 2008; 4:405-407. [PubMed: 18488014]

881. Weerapana E, Wang C, Simon GM, Richter F, Khare S, Dillon MBD, Bachovchin DA, Mowen K, Baker D, Cravatt BF. Quantitative Reactivity Profiling Predicts Functional Cysteines in Proteomes. Nature. 2010; 468:790-795. [PubMed: 21085121]

882. Qian Y, Weerapana E. A Quantitative Mass-Spectrometry Platform to Monitor Changes in Cysteine Reactivity. Methods Mol Biol. 2017; 1491:11-22. [PubMed: 27778278]

883. Abo M, Li C, Weerapana E. Isotopically-Labeled Iodoacetamide-Alkyne Probes For Quantitative Cysteine-Reactivity Profiling. Mol Pharmaceutics. 2018; 15:743-749.

884. Wang C, Weerapana E, Blewett MM, Cravatt BF. A chemoproteomic platform to quantitatively map targets of lipid-derived electrophiles. Nat Methods. 2014; 11:79-85. [PubMed: 24292485]

885. Zhou Y, Wynia-Smith SL, Couvertier SM, Kalous KS, Marletta MA, Smith BC, Weerapana E. A Chemoproteomic Strategy to Quantitatively Monitor Transnitrosation Uncovers Functionally Relevant S-Nitrosation Sites on Cathepsin D and HADH2. Cell Chem Biol. 2016; 23:727-737. [PubMed: 27291402]

886. Deng X, Weerapana E, Ulanovskaya O, Sun F, Liang H, Ji Q, Ye Y, Fu Y, Zhou L, Li J, et al. Proteome-Wide Quantification and Characterization of Oxidation-Sensitive Cysteines in Pathogenic Bacteria. Cell Host Microbe. 2013; 13:358-370. [PubMed: 23498960]

887. Abo M, Weerapana E. A Caged Electrophilic Probe for Global Analysis of Cysteine Reactivity in Living Cells. J Am Chem Soc. 2015; 137:7087-7090. [PubMed: 26020833]

888. Lin S, Yang X, Jia S, Weeks AM, Hornsby M, Lee PS, Nichiporuk RV, Iavarone AT, Wells JA, Toste FD, et al. Redox-Based Reagents for Chemoselective Methionine Bioconjugation. Science. 2017; 355:597-602. [PubMed: 28183972]

889. Rogers LK, Leinweber BL, Smith CV. Detection of Reversible Protein Thiol Modifications in Tissues. Anal Biochem. 2006; 358:171-184. [PubMed: 17007807]

890. Wong HL, Liebler DC. Mitochondrial Protein Targets of Thiol-Reactive Electrophiles. Chem Res Toxicol. 2008; 21:796-804. [PubMed: 18324786]

891. Ying J, Clavreul N, Sethuraman M, Adachi T, Cohen RA. Thiol Oxidation in Signaling and Response to Stress: Detection and Quantification of Physiological and Pathophysiological Thiol Modifications. Free Radic Biol Med. 2007; 43:1099-1108. [PubMed: 17854705]

892. Reisz JA, Bechtold E, King SB, Poole LB, Furdui CM. Thiol-Blocking Electrophiles Interfere with Labeling and Detection of Protein Sulfenic Acids. FEBS J. 2013; 280:6150-6161. [PubMed: 24103186]

893. Bar-Peled L, Kemper EK, Suciu RM, Vinogradova EV, Backus KM, Horning BD, Paul TA, Ichu T-A, Svensson RU, Olucha J, et al. Chemical Proteomics Identifies Druggable Vulnerabilities in a Genetically Defined Cancer. Cell. 2017; 171:696-709.e623. [PubMed: 28965760]

894. Abo M, Bak DW, Weerapana E. Optimization of Caged Electrophiles for Improved Monitoring of Cysteine Reactivity in Living Cells. ChemBioChem. 2017; 18:81-84. [PubMed: 27813293]

895. Bak DW, Pizzagalli MD, Weerapana E. Identifying Functional Cysteine Residues in the Mitochondria. ACS Chem Biol. 2017; 12:947-957. [PubMed: 28157297] 
896. Poole LB, Zeng BB, Knaggs SA, Yakubu M, King SB. Synthesis of Chemical Probes to Map Sulfenic Acid Modifications on Proteins. Bioconjugate Chem. 2005; 16:1624-1628.

897. Seo YH, Carroll KS. Quantification of Protein Sulfenic Acid Modifications Using Isotope-Coded Dimedone And Iododimedone. Angew Chem Int Ed. 2011; 50:1342-1345.

898. Yang J, Gupta V, Tallman KA, Porter NA, Carroll KS, Liebler DC. Global, in Situ, Site-Specific Analysis of Protein S-Sulfenylation. Nat Protocols. 2015; 10:1022-1037. [PubMed: 26086405]

899. Furdui CM, Poole LB. Chemical Approaches to Detect and Analyze Protein Sulfenic Acids. Mass Spectrom Rev. 2014; 33:126-146. [PubMed: 24105931]

900. Benitez LV, Allison WS. The Inactivation of the Acyl Phosphatase Activity Catalyzed by the Sulfenic Acid form of Glyceraldehyde 3-Phosphate Dehydrogenase by Dimedone and Olefins. J Biol Chem. 1974; 249:6234-6243. [PubMed: 4371119]

901. Poole LB, Klomsiri C, Knaggs SA, Furdui CM, Nelson KJ, Thomas MJ, Fetrow JS, Daniel LW, King SB. Fluorescent and Affinity-Based Tools to Detect Cysteine Sulfenic Acid Formation in Proteins. Bioconjugate Chem. 2007; 18:2004-2017.

902. Charles RL, Schröder E, May G, Free P, Gaffney PRJ, Wait R, Begum S, Heads RJ, Eaton P. Protein Sulfenation as a Redox Sensor: Proteomics Studies Using a Novel Biotinylated Dimedone Analogue. Mol Cell Proteomics. 2007; 6:1473-1484. [PubMed: 17569890]

903. Seo YH, Carroll KS. Facile Synthesis and Biological Evaluation of a Cell-Permeable Probe to Detect Redox-Regulated Proteins. Biorg Med Chem Lett. 2009; 19:356-359.

904. Reddie KG, Seo YH, Muse WB, Leonard SE, Carroll KS. A Chemical Approach for Detecting Sulfenic Acid-Modified Proteins in Living Cells. Mol Biosyst. 2008; 4:521-531. [PubMed: 18493649]

905. Paulsen CE, Truong TH, Garcia FJ, Homann A, Gupta V, Leonard SE, Carroll KS. Peroxidedependent sulfenylation of the EGFR catalytic site enhances kinase activity. Nature chemical biology. 2011; 8:57-64. [PubMed: 22158416]

906. Klomsiri C, Nelson KJ, Bechtold E, Soito L, Johnson LC, Lowther WT, Ryu SE, King SB, Furdui CM, Poole LB. Use of Dimedone-Based Chemical Probes for Sulfenic Acid Detection: Evaluation of Conditions Affecting Probe Incorporation into Redox-Sensitive Proteins. Methods Enzymol. 2010; 473:77-94. [PubMed: 20513472]

907. Forman HJ, Davies MJ, Krämer AC, Miotto G, Zaccarin M, Zhang H, Ursini F. Protein Cysteine Oxidation in Redox Signaling: Caveats on Sulfenic Acid Detection and Quantification. Arch Biochem Biophys. 2017; 617:26-37. [PubMed: 27693037]

908. Michalek RD, Nelson KJ, Holbrook BC, Yi JS, Stridiron D, Daniel LW, Fetrow JS, King SB, Poole LB, Grayson JM. The Requirement of Reversible Cysteine Sulfenic Acid Formation for T Cell Activation and Function. J Immunol. 2007; 179:6456-6467. [PubMed: 17982034]

909. Kaplan N, Urao N, Furuta E, Kim SJ, Razvi M, Nakamura Y, Mckinney RD, Poole LB, Fukai T, Ushio-Fukai M. Localized Cysteine Sulfenic Acid Formation by Vascular Endothelial Growth Factor: Role in Endothelial Cell Migration and Angiogenesis. Free Radic Res. 2011; 45:11241135. [PubMed: 21740309]

910. Leonard SE, Reddie KG, Carroll KS. Mining the Thiol Proteome for Sulfenic Acid Modifications Reveals New Targets for Oxidation in Cells. ACS Chem Biol. 2009; 4:783-799. [PubMed: 19645509]

911. Truong TH, Ung PMU, Palde PB, Paulsen CE, Schlessinger A, Carroll KS. Molecular Basis for Redox Activation of Epidermal Growth Factor Receptor Kinase. Cell chemical biology. 2016; 23:837-848. [PubMed: 27427230]

912. Truong TH, Garcia FJ, Seo YH, Carroll KS. Isotope-Coded Chemical Reporter and AcidCleavable Affinity Reagents for Monitoring Protein Sulfenic Acids. Biorg Med Chem Lett. 2011; 21:5015-5020.

913. Yang J, Gupta V, Carroll KS, Liebler DC. Site-Specific Mapping and Quantification of Protein SSulfenylation in Cells. Nature communications. 2014; 5:4776-4776.

914. Poole TH, Reisz JA, Zhao W, Poole LB, Furdui CM, King SB. Strained Cycloalkynes as New Protein Sulfenic Acid Traps. J Am Chem Soc. 2014; 136:6167-6170. [PubMed: 24724926] 
915. Qian J, Klomsiri C, Wright MW, King SB, Tsang AW, Poole LB, Furdui CM. Simple Synthesis of 1,3-Cyclopentanedione Derived Probes for Labeling Sulfenic Acid Proteins. Chem Commun (Cambridge, U K). 2011; 47:9203-9205.

916. Qian J, Wani R, Klomsiri C, Poole LB, Tsang AW, Furdui CM. A Simple and Effective Strategy for Labeling Cysteine Sulfenic Acid in Proteins by Utilization of $\beta$-Ketoesters as Cleavable Probes. Chem Commun (Cambridge, U K). 2012; 48:4091-4093.

917. Gupta V, Carroll KS. Profiling the Reactivity of Cyclic C-Nucleophiles Towards Electrophilic Sulfur in Cysteine Sulfenic Acid. Chem Sci. 2016; 7:400-415. [PubMed: 26819701]

918. Gupta V, Yang J, Liebler DC, Carroll KS. Diverse Redoxome Reactivity Profiles of Carbon Nucleophiles. J Am Chem Soc. 2017; 139:5588-5595. [PubMed: 28355876]

919. Ellis HR, Poole LB. Novel Application of 7-Chloro-4-Nitrobenzo-2-Oxa-1,3-Diazole to Identify Cysteine Sulfenic Acid in the ahpc Component of Alkyl Hydroperoxide Reductase. Biochemistry. 1997; 36:15013-15018. [PubMed: 9398227]

920. Denu JM, Tanner KG. Specific and Reversible Inactivation of Protein Tyrosine Phosphatases by Hydrogen Peroxide:w Evidence for a Sulfenic Acid Intermediate and Implications for Redox Regulation. Biochemistry. 1998; 37:5633-5642. [PubMed: 9548949]

921. Carballal S, Alvarez B, Turell L, Botti H, Freeman BA, Radi R. Sulfenic Acid in Human Serum Albumin. Amino Acids. 2007; 32:543-551. [PubMed: 17061035]

922. Griffiths SW, King J, Cooney CL. The Reactivity and Oxidation Pathway of Cysteine 232 in Recombinant Human a1-Antitrypsin. J Biol Chem. 2002; 277:25486-25492. [PubMed: 11991955]

923. Shetty V, Spellman DS, Neubert TA. Characterization by Tandem Mass Spectrometry of Stable Cysteine Sulfenic Acid in a Cysteine Switch Peptide of Matrix Metalloproteinases. J Am Soc Mass Spectrom. 2007; 18:1544-1551. [PubMed: 17604642]

924. Chang YC, Huang CN, Lin CH, Chang HC, Wu CC. Mapping Protein Cysteine Sulfonic Acid Modifications with Specific Enrichment and Mass Spectrometry: An Integrated Approach to Explore the Cysteine Oxidation. Proteomics. 2010; 10:2961-2971. [PubMed: 20629170]

925. Wagner E, Luche S, Penna L, Chevallet M, Van Dorsselaer A, Leize-Wagner E, Rabilloud T. A Method for Detection of Overoxidation of Cysteines: Peroxiredoxins are Oxidized in Vivo at the Active-Site Cysteine During Oxidative Stress. Biochem J. 2002; 366:777-785. [PubMed: 12059788]

926. Jeong J, Jung Y, Na S, Jeong J, Lee E, Kim M-S, Choi S, Shin D-H, Paek E, Lee H-Y, et al. Novel Oxidative Modifications in Redox-Active Cysteine Residues. Mol Cell Proteomics. 2011; 10:M110.000513.

927. Burkhard RK, Sellers DE, Decou F, Lambert JL. The pKa's of Aromatic Sulfinic Acids. J Org Chem. 1959; 24:767-769.

928. Dai J, Wang J, Zhang Y, Lu Z, Yang B, Li X, Cai Y, Qian X. Enrichment and Identification of Cysteine-Containing Peptides from Tryptic Digests of Performic Oxidized Proteins by Strong Cation Exchange LC and MALDI-TOF/TOF MS. Anal Chem. 2005; 77:7594-7604. [PubMed: 16316166]

929. Paulech J, Liddy KA, Engholm-Keller K, White MY, Cordwell SJ. Global Analysis of Myocardial Peptides Containing Cysteines with Irreversible Sulfinic and Sulfonic Acid Post-Translational Modifications. Mol Cell Proteomics. 2015; 14:609-620. [PubMed: 25561502]

930. Bell RM, Mocanu MM, Yellon DM. Retrograde Heart Perfusion: The Langendorff Technique of Isolated Heart Perfusion. J Mol Cell Cardiol. 2011; 50:940-950. [PubMed: 21385587]

931. Murray CI, Van Eyk JE. Chasing Cysteine Oxidative Modifications: Proteomic Tools for Characterizing Cysteine Redox-Status. Circ Cardiovasc Genet. 2012; 5:591-591. [PubMed: 23074338]

932. Lo Conte M, Carroll KS. Chemoselective Ligation of Sulfinic Acids with Aryl-Nitroso Compounds. Angew Chem Int Ed. 2012; 51:6502-6505.

933. Lo Conte M, Lin J, Wilson MA, Carroll KS. A Chemical Approach for the Detection of Protein Sulfinylation. ACS Chem Biol. 2015; 10:1825-1830. [PubMed: 26039147] 
934. Tarrago L, Péterfi Z, Lee BC, Michel T, Gladyshev VN. Monitoring Methionine Sulfoxide with Stereospecific Mechanism-Based Fluorescent Sensors. Nat Chem Biol. 2015; 11:332-338. [PubMed: 25799144]

935. Hensley K. Detection of Blotted Proteins: Methods and Protocols. Kurien BT, Scofield RH, editorsSpringer New York; New York, NY: 2015.

936. Mateos R, Lecumberri E, Ramos S, Goya L, Bravo L. Determination of Malondialdehyde (MDA) by High-Performance Liquid Chromatography in Serum and Liver as a Biomarker for Oxidative Stress: Application to a Rat Model for Hypercholesterolemia and Evaluation of the Effect of Diets Rich in Phenolic Antioxidants from Fruits. J Chromatogr B: Anal Technol Biomed Life Sci. 2005; 827:76-82.

937. Siegel D, Meinema AC, Permentier H, Hopfgartner G, Bischoff R. Integrated Quantification and Identification of Aldehydes and Ketones in Biological Samples. Anal Chem. 2014; 86:50895100. [PubMed: 24745975]

938. Tamarit J, De Hoogh A, Obis E, Alsina D, Cabiscol E, Ros J. Analysis of Oxidative StressInduced Protein Carbonylation Using Fluorescent Hydrazides. J Proteomics. 2012; 75:37783788. [PubMed: 22579746]

939. Maier CS, Chavez J, Wang J, Wu J. Protein Adducts of Aldehydic Lipid Peroxidation Products: Identification and Characterization of Protein Adducts Using an Aldehyde/Keto Reactive Probe in Combination with Mass Spectrometry. Methods Enzymol. 2010; 473:305-330. [PubMed: 20513485]

940. Soreghan BA, Yang F, Thomas SN, Hsu J, Yang AJ. High-Throughput Proteomic-Based Identification of Oxidatively Induced Protein Carbonylation in Mouse Brain. Pharm Res. 2003; 20:1713-1720. [PubMed: 14661913]

941. Codreanu SG, Zhang B, Sobecki SM, Billheimer DD, Liebler DC. Global Analysis of Protein Damage by the Lipid Electrophile 4-Hydroxy-2-Nonenal. Mol Cell Proteomics. 2009; 8:670 680. [PubMed: 19054759]

942. Galligan JJ, Smathers RL, Fritz KS, Epperson LE, Hunter LE, Petersen DR. Protein Carbonylation in a Murine Model for Early Alcoholic Liver Disease. Chem Res Toxicol. 2012; 25:1012-1021. [PubMed: 22502949]

943. Chen Y, Cong Y, Quan B, Lan T, Chu X, Ye Z, Hou X, Wang C. Chemoproteomic Profiling of Targets of Lipid-Derived Electrophiles by Bioorthogonal Aminooxy Probe. Redox Biol. 2017; 12:712-718. [PubMed: 28411555]

944. Chen Y, Liu Y, Lan T, Qin W, Zhu Y, Qin K, Gao J, Wang H, Hou X, Chen N, et al. Quantitative Profiling of Protein Carbonylations in Ferroptosis by an Aniline-Derived Probe. J Am Chem Soc. 2018; 140:4712-4720. [PubMed: 29569437]

945. Dalle-Donne I, Carini M, Orioli M, Vistoli G, Regazzoni L, Colombo G, Rossi R, Milzani A, Aldini G. Protein Carbonylation: 2,4-Dinitrophenylhydrazine Reacts with both Aldehydes/ Ketones and Sulfenic Acids. Free Radical Biol Med. 2009; 46:1411-1419. [PubMed: 19268703]

946. Landar A, Zmijewski JW, Dickinson DA, Le Goffe C, Johnson MS, Milne GL, Zanoni G, Vidari G, Morrow JD, Darley-Usmar VM. Interaction of Electrophilic Lipid Oxidation Products with Mitochondria in Endothelial Cells and Formation of Reactive Oxygen Species. Am J Physiol Heart Circ Physiol. 2006; 290:H1777-H1787. [PubMed: 16387790]

947. Zmijewski JW, Landar A, Watanabe N, Dickinson DA, Noguchi N, Darley-Usmar VM. Cell Signalling by Oxidized Lipids and the Role of Reactive Oxygen Species in the Endothelium. Biochem Soc Trans. 2005; 33:1385-1389. [PubMed: 16246125]

948. Higdon AN, Dranka BP, Hill BG, Oh JY, Johnson MS, Landar A, Darley-Usmar VM. Methods for Imaging and Detecting Modification of Proteins by Reactive Lipid Species. Free Radical Biol Med. 2009; 47:201-212. [PubMed: 19446632]

949. Landar A, Shiva S, Levonen AL, Oh JY, Zaragoza C, Johnson Michelle s, Darley-Usmar Victor m. Induction of the Permeability Transition and Cytochrome C Release by 15 -Deoxy- $\Delta(12,14)-$ Prostaglandin (2) in Mitochondria. Biochem J. 2006; 394:185-195. [PubMed: 16268779]

950. Tallman KA, Kim HYH, Ji JX, Szapacs ME, Yin H, Mcintosh TJ, Liebler DC, Porter NA. Phospholipid-Protein Adducts of Lipid Peroxidation:w Synthesis and Study of New Biotinylated Phosphatidylcholines. Chem Res Toxicol. 2007; 20:227-234. [PubMed: 17305406] 
951. Szapacs ME, Kim HYH, Porter NA, Liebler DC. Identification of Proteins Adducted by Lipid Peroxidation Products in Plasma and Modifications of Apolipoprotein A1 with a Novel Biotinylated Phospholipid Probe. J Proteome Res. 2008; 7:4237-4246. [PubMed: 18778096]

952. Lincoln R, Greene LE, Zhang W, Louisia S, Cosa G. Mitochondria Alkylation and Cellular Trafficking Mapped with a Lipophilic Bodipy-Acrolein Fluorogenic Probe. J Am Chem Soc. 2017; 139:16273-16281. [PubMed: 28976196]

953. Vila A, Tallman KA, Jacobs AT, Liebler DC, Porter NA, Marnett LJ. Identification of Protein Targets of 4-Hydroxynonenal Using Click Chemistry for Ex Vivo Biotinylation of Azido and Alkynyl Derivatives. Chem Res Toxicol. 2008; 21:432-444. [PubMed: 18232660]

954. Codreanu SG, Ullery JC, Zhu J, Tallman KA, Beavers WN, Porter NA, Marnett LJ, Zhang B, Liebler DC. Alkylation Damage by Lipid Electrophiles Targets Functional Protein Systems. Mol Cell Proteomics. 2014; 13:849-859. [PubMed: 24429493]

955. Kim HYH, Tallman KA, Liebler DC, Porter NA. An Azido-Biotin Reagent for Use in the Isolation of Protein Adducts of Lipid-Derived Electrophiles by Streptavidin Catch and Photorelease. Mol Cell Proteomics. 2009; 8:2080-2089. [PubMed: 19483245]

956. Los GV, Encell LP, Mcdougall MG, Hartzell DD, Karassina N, Zimprich C, Wood MG, Learish R, Ohana RF, Urh M, et al. HaloTag: A Novel Protein Labeling Technology for Cell Imaging and Protein Analysis. ACS Chem Biol. 2008; 3:373-382. [PubMed: 18533659]

957. Fang X, Fu Y, Long MJC, Haegele JA, Ge EJ, Parvez S, Aye Y. Temporally Controlled Targeting of 4-Hydroxynonenal to Specific Proteins in Living Cells. J Am Chem Soc. 2013; 135:1449614499. [PubMed: 24015839]

958. Long MJC, Poganik JR, Ghosh S, Aye Y. Subcellular Redox Targeting: Bridging in Vitro and in Vivo Chemical Biology. ACS Chem Biol. 2017; 12:586-600. [PubMed: 28068059]

959. Parvez S, Long MJC, Lin HY, Zhao Y, Haegele JA, Pham VN, Lee DK, Aye Y. T-REX ondemand redox targeting in live cells. Nat Protocols. 2016; 11:2328-2356. [PubMed: 27809314]

960. Long MJC, Urul DA, Chawla S, Lin HY, Zhao Y, Haegele JA, Wang Y, Aye Y. Precision Electrophile Tagging in Caenorhabditis elegans. Biochemistry. 2017; 57:216-220. [PubMed: 28857552]

961. Long MJ, Poganik JR, Aye Y. On-Demand Targeting: Investigating Biology with ProximityDirected Chemistry. J Am Chem Soc. 2016; 138:3610-3622. [PubMed: 26907082]

962. Van Hall-Beauvais A, Zhao Y, Urul DA, Long MJC, Aye Y. Single-Protein-Specific Redox Targeting in Live Mammalian Cells and C. elegans. Curr Protoc Chem Biol. 2018; In Press. doi: 10.1002/cpch.43

963. Holland R, Fishbein JC. Chemistry of the Cysteine Sensors in Kelch-Like ECH-Associated Protein 1. Antioxid Redox Signal. 2010; 13:1749-1761. [PubMed: 20486763]

964. Surya SL, Long MJC, Urul DA, Zhao Y, Mercer EJ, Eisaid IM, Evans T, Aye Y. Cardiovascular Small Heat Shock Protein HSPB7 is a Kinetically Privileged Reactive Electrophilic Species (RES) Sensor. ACS Chem Biol. 2018; 13:1824-1831. [PubMed: 29397684]

965. Urh M, Rosenberg M. HaloTag, a Platform Technology for Protein Analysis. Curr Chem Genomics. 2012; 6:72-78. [PubMed: 23213345]

966. England CG, Luo H, Cai W. HaloTag Technology: A Versatile Platform for Biomedical Applications. Bioconjugate Chem. 2015; 26:975-986.

967. Nakamura A, Goto S. Analysis of Protein Carbonyls with 2, 4-Dinitrophenyl Hydrazine and its Antibodies by Immunoblot in Two-Dimensional Gel Electrophoresis. J Biochem. 1996; 119:768774. [PubMed: 8743580]

968. Levine RL, Wehr N, Williams JA, Stadtman ER, Shacter E. Stress Response: Methods and Protocols. Walker JM, Keyse SM, editorsHumana Press; Totowa, NJ: 2000.

969. Stankowski J, Codreanu S, Liebler D, Mclaughlin B. Analysis of Protein Targets by Oxidative Stress Using the Oxyblot and Biotin-Avidin-Capture Methodology. Human Press; New York: 2011.

970. Baraibar MA, Hyzewicz J, Rogowska-Wrzesinska A, Bulteau A-L, Prip-Buus C, Butler-Browne G, Friguet B. Impaired Energy Metabolism of Senescent Muscle Satellite Cells is Associated with Oxidative Modifications of Glycolytic Enzymes. Aging (N Y). 2016; 8:3375-3388. 
971. Fenaille F, Tabet JC, Guy PA. Immunoaffinity Purification and Characterization of 4-Hydroxy-2nonenal- and Malondialdehyde-Modified Peptides by Electrospray Ionization Tandem Mass Spectrometry. Anal Chem. 2002; 74:6298-6304. [PubMed: 12510752]

972. Le Boulch M, Ahmed EK, Rogowska-Wrzesinska A, Baraibar MA, Friguet B. Proteome Oxidative Carbonylation During Oxidative Stress-Induced Premature Senescence of WI-38 Human Fibroblasts. Mech Ageing Dev. 2017; 170:59-71. [PubMed: 28757326]

973. Seo YH, Carroll KS. Profiling Protein Thiol Oxidation in Tumor Cells Using Sulfenic AcidSpecific Antibodies. Proc Natl Acad Sci U S A. 2009; 106:16163-16168. [PubMed: 19805274]

974. Paulsen CE, Truong TH, Garcia FJ, Homann A, Gupta V, Leonard SE, Carroll KS. Peroxidedependent sulfenylation of the EGFR catalytic site enhances kinase activity. Nature Chemical Biology. 2011; 8:57. [PubMed: 22158416]

975. Maller C, Schröder E, Eaton P. Glyceraldehyde 3-Phosphate Dehydrogenase is Unlikely to Mediate Hydrogen Peroxide Signaling: Studies with a Novel Anti-Dimedone Sulfenic Acid Antibody. Antioxid Redox Signal. 2010; 14:49-60. [PubMed: 20518697]

976. Persson C, Kappert K, Engström U, Östman A, Sjöblom T. An Antibody-Based Method for Monitoring in Vivo Oxidation of Protein Tyrosine Phosphatases. Methods. 2005; 35:37-43. [PubMed: 15588984]

977. Haque A, Andersen JN, Salmeen A, Barford D, Tonks NK. Conformation-Sensing Antibodies Stabilize the Oxidized Form of PTP1B and Inhibit its Phosphatase Activity. Cell. 2011; 147:185198. [PubMed: 21962515]

978. Woo HA, Won Kang S, Kim HK, Yang KS, Chae HZ, Rhee SG. Reversible Oxidation of the Active Site Cysteine of Peroxiredoxins to Cysteine Sulfinic Acid: Immunoblot Detection with Antibodies Specific for the Hyperoxidized Cysteine-Containing Sequence. J Biol Chem. 2003; 278:47361-47364. [PubMed: 14559909]

979. Uchida K, Szweda LI, Chae HZ, Stadtman ER. Immunochemical Detection of 4-Hydroxynonenal Protein Adducts in Oxidized Hepatocytes. Proc Natl Acad Sci U S A. 1993; 90:8742-8746. [PubMed: 8378358]

980. Neely MD, Sidell KR, Graham DG, Montine TJ. The Lipid Peroxidation Product 4Hydroxynonenal Inhibits Neurite Outgrowth, Disrupts Neuronal Microtubules, and Modifies Cellular Tubulin. J Neurochem. 1999; 72:2323-2333. [PubMed: 10349841]

981. Tanito M, Elliott MH, Kotake Y, Anderson RE. Protein Modifications by 4-Hydroxynonenal and 4-Hydroxyhexenal in Light-Exposed Rat Retina. Invest Ophthalmol Vis Sci. 2005; 46:38593868. [PubMed: 16186375]

982. Hartley DP, Kroll DJ, Petersen DR. Prooxidant-Initiated Lipid Peroxidation in Isolated Rat Hepatocytes:w Detection of 4-Hydroxynonenal- and Malondialdehyde-Protein Adducts. Chem Res Toxicol. 1997; 10:895-905. [PubMed: 9282839]

983. Hartley DP, Kolaja KL, Reichard J, Petersen DR. 4-Hydroxynonenal and Malondialdehyde Hepatic Protein Adducts in Rats Treated with Carbon Tetrachloride: Immunochemical Detection and Lobular Localization. Toxicol Appl Pharmacol. 1999; 161:23-33. [PubMed: 10558920]

984. Uchida K. Current Status of Acrolein as a Lipid Peroxidation Product. Trends Cardiovasc Med. 1999; 9:109-113. [PubMed: 10639724]

985. Uchida K, Kanematsu M, Sakai K, Matsuda T, Hattori N, Mizuno Y, Suzuki D, Miyata T, Noguchi N, Niki E, et al. Protein-Bound Acrolein: Potential Markers for Oxidative Stress. Proc Natl Acad Sci U S A. 1998; 95:4882-4887. [PubMed: 9560197]

986. Nadkarni DV, Sayre LM. Structural Definition of Early Lysine and Histidine Adduction Chemistry of 4-Hydroxynonenal. Chem Res Toxicol. 1995; 8:284-291. [PubMed: 7766813]

987. Sayre LM, Sha W, Xu G, Kaur K, Nadkarni D, Subbanagounder G, Salomon RG. Immunochemical Evidence Supporting 2-Pentylpyrrole Formation on Proteins Exposed to 4Hydroxy-2-Nonenal. Chem Res Toxicol. 1996; 9:1194-1201. [PubMed: 8902276]

988. Gu X, Meer SG, Miyagi M, Rayborn ME, Hollyfield JG, Crabb JW, Salomon RG. Carboxyethylpyrrole Protein Adducts and Autoantibodies, Biomarkers for Age-Related Macular Degeneration. J Biol Chem. 2003; 278:42027-42035. [PubMed: 12923198]

989. Charles RL, Burgoyne JR, Mayr M, Weldon SM, Hubner N, Dong H, Morisseau C, Hammock BD, Landar AL, Eaton P. Redox Regulation of Soluble Epoxide Hydrolase by 15-Deoxy- $\Delta$ - 
Prostaglandin (2) Controls Coronary Hypoxic Vasodilation. Circ Res. 2011; 108:324-334. [PubMed: 21164107]

990. Uchida K, Itakura K, Kawakishi S, Hiai H, Toyokuni S, Stadtman ER. Characterization of Epitopes Recognized by 4-Hydroxy-2-nonenal Specific Antibodies. Arch Biochem Biophys. 1995; 324:241-248. [PubMed: 8554315]

991. Baynes JW, Thorpe SR. Role of Oxidative Stress in Diabetic Complications: A New Perspective on an Old Paradigm. Diabetes. 1999; 48:1-9. [PubMed: 9892215]

992. Kaneto H, Katakami N, Matsuhisa M, Matsuoka TA. Role of Reactive Oxygen Species in the Progression of Type 2 Diabetes and Atherosclerosis. Mediatiors Inflammation. 2010; 2010:453892.

993. Son Y, Kim S, Chung H-T, Pae H-O. Methods Enzymol. Cadenas E, Packer L, editors Vol. 528. Academic Press; 2013.

994. Yoshizumi M, Abe JI, Haendeler J, Huang Q, Berk BC. Src and Cas Mediate JNK Activation But Not ERK1/2 and p38 Kinases by Reactive Oxygen Species. J Biol Chem. 2000; 275:1170611712. [PubMed: 10766791]

995. Frohnert BI, Sinaiko AR, Serrot FJ, Foncea RE, Moran A, Ikramuddin S, Choudry U, Bernlohr DA. increased Adipose Protein Carbonylation in Human Obesity. Obesity. 2011; 19:1735-1741. [PubMed: 21593812]

996. Guo L, Zhang XM, Zhang YB, Huang X, Chi MH. Association of 4-Hydroxynonenal with Classical Adipokines and Insulin Resistance in a Chinese Non-Diabetic Obese Population. Nutr Hosp. 2017; 34:363-368. [PubMed: 28421791]

997. Curtis JM, Grimsrud PA, Wright WS, Xu X, Foncea RE, Graham DW, Brestoff JR, Wiczer BM, Ilkayeva O, Cianflone K, et al. Downregulation of Adipose Glutathione S-Transferase A4 Leads to Increased Protein Carbonylation, Oxidative Stress, and Mitochondrial Dysfunction. Diabetes. 2010; 59:1132-1142. [PubMed: 20150287]

998. Traverso N, Menini S, Odetti P, Pronzato MA, Cottalasso D, Marinari UM. Diabetes Impairs the Enzymatic Disposal of 4-Hydroxynonenal in Rat Liver. Free Radical Biol Med. 2002; 32:350359. [PubMed: 11841925]

999. Elrayess MA, Almuraikhy S, Kafienah W, Al-Menhali A, Al-Khelaifi F, Bashah M, Zarkovic K, Zarkovic N, Waeg G, Alsayrafi M, et al. 4-Hydroxynonenal Causes Impairment of Human Subcutaneous Adipogenesis and Induction of Adipocyte Insulin Resistance. Free Radical Biol Med. 2017; 104:129-137. [PubMed: 28088621]

1000. Fatani SH, Babakr AT, Noureldin EM, Almarzouki AA. Lipid Peroxidation is Associated with Poor Control of Type-2 Diabetes Mellitus. Diabetes Metab Syndr. 2016; 10:S64-S67. [PubMed: 26806326]

1001. Chin M, Lee CYI, Chuang JC, Bumeister R, Wigley WC, Sonis ST, Ward KW, Meyer C. Bardoxolone Methyl Analogs RTA 405 and dh404 are Well Tolerated and Exhibit Efficacy in Rodent Models of Type 2 Diabetes and Obesity. Am J Physiol Renal Physiol. 2013; 304:F1438F1446. [PubMed: 23594825]

1002. Himmelfarb J, Tuttle KR. New Therapies for Diabetic Kidney Disease. New Engl J Med. 2013; 369:2549-2550. [PubMed: 24206460]

1003. De Zeeuw D, Akizawa T, Audhya P, Bakris GL, Chin M, Christ-Schmidt H, Goldsberry A, Houser M, Krauth M, Lambers Heerspink HJ, et al. Bardoxolone Methyl in Type 2 Diabetes and Stage 4 Chronic Kidney Disease. New Engl J Med. 2013; 369:2492-2503. [PubMed: 24206459]

1004. Chartoumpekis DV, Sykiotis GP. Bardoxolone Methyl in Type 2 Diabetes and Advanced Chronic Kidney Disease. New Engl J Med. 2014; 370:1767-1769.

1005. Shahzad K, Bock F, Al-Dabet MDM, Gadi I, Nazir S, Wang H, Kohli S, Ranjan S, Mertens PR, Nawroth PP, et al. Stabilization of Endogenous Nrf2 by Minocycline Protects Against Nlrp3Inflammasome Induced Diabetic Nephropathy. Sci Rep. 2016; 6:34228. [PubMed: 27721446]

1006. Wong MHL, Bryan HK, Copple IM, Jenkins RE, Chiu PH, Bibby J, Berry NG, Kitteringham NR, Goldring CE, O'neill PM, et al. Design and Synthesis of Irreversible Analogues of Bardoxolone Methyl for the Identification of Pharmacologically Relevant Targets and Interaction Sites. J Med Chem. 2016; 59:2396-2409. [PubMed: 26908173] 
1007. Kim GH, Kim JE, Rhie SJ, Yoon S. The Role of Oxidative Stress in Neurodegenerative Diseases. Exp Neurobiol. 2015; 24:325-340. [PubMed: 26713080]

1008. Uttara B, Singh AV, Zamboni P, Mahajan RT. Oxidative Stress and Neurodegenerative Diseases: A Review of Upstream and Downstream Antioxidant Therapeutic Options. Curr Neuropharmacol. 2009; 7:65-74. [PubMed: 19721819]

1009. Jenner P. Oxidative Stress in Parkinson's Disease. Ann Neurol. 2003; 53:S26-S38. [PubMed: 12666096]

1010. Ellis G, Fang E, Maheshwari M, Roltsch E, Holcomb L, Zimmer D, Martinez D, Murray IV. Lipid Oxidation and Modification of Amyloid-Beta (A $\beta$ ) in Vitro And in Vivo. J Alzheimers Dis. 2010; 22:593-607. [PubMed: 20847409]

1011. Ryberg H, Söderling AS, Davidsson P, Blennow K, Caidahl K, Persson LI. Cerebrospinal Fluid Levels of Free 3-Nitrotyrosine Are Not Elevated in the Majority of Patients with Amyotrophic Lateral Sclerosis or Alzheimer's Disease. Neurochem Int. 2004; 45:57-62. [PubMed: 15082222]

1012. Van Raamsdonk JM, Vega IE, Brundin P. Oxidative Stress in Neurodegenerative Disease: Causation or Association? Oncotarget. 2017; 8:10777-10778. [PubMed: 28099897]

1013. Branca C, Ferreira E, Nguyen TV, Doyle K, Caccamo A, Oddo S. Genetic Reduction of Nrf2 Exacerbates Cognitive Deficits in a Mouse Model of Alzheimer's Disease. Hum Mol Genet. 2017; 26:4823-4835. [PubMed: 29036636]

1014. Rojo AI, Pajares M, Rada P, Nuñez A, Nevado-Holgado AJ, Killik R, Van Leuven F, Ribe E, Lovestone S, Yamamoto M, et al. NRF2 Deficiency Replicates Transcriptomic Changes in Alzheimer's Patients and Worsens APP and TAU Pathology. Redox Biol. 2017; 13:444-451. [PubMed: 28704727]

1015. Shrestha A, Megeney LA. Yeast Proteinopathy Models: A Robust Tool for Deciphering the Basis of Neurodegeneration. Microb Cell. 2015; 2:458-465. [PubMed: 28357271]

1016. Gitler AD, Dhillon P, Shorter J. Neurodegenerative Disease: Models, Mechanisms, and a New Hope. Dis Model Mech. 2017; 10:499-502. [PubMed: 28468935]

1017. Marton Rebecca mPa $\bullet$ ca Sergiu p. Neural Differentiation in the Third Dimension: Generating a Human Midbrain. Cell Stem Cell. 2016; 19:145-146. [PubMed: 27494668]

1018. Pedersen JT, Chen SW, Borg CB, Ness S, Bahl JM, Heegaard NHH, Dobson CM, Hemmingsen L, Cremades N, Teilum K. Amyloid- $\beta$ and $\alpha$-Synuclein Decrease the Level of Metal-Catalyzed Reactive Oxygen Species by Radical Scavenging and Redox Silencing. J Am Chem Soc. 2016; 138:3966-3969. [PubMed: 26967463]

1019. Deas E, Cremades N, Angelova PR, Ludtmann MHR, Yao Z, Chen S, Horrocks MH, Banushi B, Little D, Devine MJ, et al. Alpha-Synuclein Oligomers Interact with Metal Ions to Induce Oxidative Stress and Neuronal Death in Parkinson's Disease. Antioxid Redox Signal. 2016; 24:376-391. [PubMed: 26564470]

1020. Liu L, Zhang K, Sandoval H, Yamamoto S, Jaiswal M, Sanz E, Li Z, Hui J, Graham Brett h, Quintana A, et al. Glial Lipid Droplets and ROS Induced by Mitochondrial Defects Promote Neurodegeneration. Cell. 2015; 160:177-190. [PubMed: 25594180]

1021. Spencer NG, Schilling T, Miralles F, Eder C. Mechanisms Underlying Interferon- $\boldsymbol{\gamma}$-Induced Priming of Microglial Reactive Oxygen Species Production. PLoS One. 2016; 11:e0162497. [PubMed: 27598576]

1022. Rojas F, Gonzalez D, Cortes N, Ampuero E, Hernández DE, Fritz E, Abarzua S, Martinez A, Elorza AA, Alvarez A, et al. Reactive Oxygen Species Trigger Motoneuron Death in Non-CellAutonomous Models of ALS through Activation of c-Abl Signaling. Front Cell Neurosci. 2015; 9:203. [PubMed: 26106294]

1023. Ci Y, Shi K, An J, Yang Y, Hui K, Wu P, Shi L, Xu C. ROS Inhibit Autophagy by Downregulating ULK1 Mediated by the Phosphorylation of p53 in Selenite-Treated NB4 Cells. Cell Death Dis. 2014; 5:e1542. [PubMed: 25429619]

1024. Fischer R, Maier O. Interrelation of Oxidative Stress and Inflammation in Neurodegenerative Disease: Role of TNF. Oxid Med Cell Longev. 2015; 2015:18.

1025. Dasuri K, Zhang L, Keller JN. Oxidative Stress, Neurodegeneration, and the Balance of Protein Degradation and Protein Synthesis. Free Radical Biol Med. 2013; 62:170-185. [PubMed: 23000246] 
1026. Velusamy T, Panneerselvam AS, Purushottam M, Anusuyadevi M, Pal PK, Jain S, Essa MM, Guillemin GJ, Kandasamy M. Protective Effect of Antioxidants on Neuronal Dysfunction and Plasticity in Huntington's Disease. Oxid Med Cell Longev. 2017; 2017:3279061. [PubMed: 28168008]

1027. Rebec GV, Barton SJ, Marseilles AM, Collins K. Ascorbate Treatment Attenuates the Huntington Behavioral Phenotype in Mice. NeuroReport. 2003; 14:1263-1265. [PubMed: 12824772]

1028. Andreassen OA, Ferrante RJ, Dedeoglu A, Beal MF. Lipoic Acid Improves Survival in Transgenic Mouse Models of Huntington's Disease. Neuroreport. 2001; 12:3371-3373. [PubMed: 11711888]

1029. Van Raamsdonk JM, Pearson J, Bailey CDC, Rogers DA, Johnson GVW, Hayden MR, Leavitt BR. Cystamine Treatment is Neuroprotective in the YAC128 Mouse Model of Huntington Disease. J Neurochem. 2005; 95:210-220. [PubMed: 16181425]

1030. Ferrante RJ, Andreassen OA, Dedeoglu A, Ferrante KL, Jenkins BG, Hersch SM, Beal MF. Therapeutic Effects of Coenzyme $\mathrm{Q}_{10}$ and Remacemide in Transgenic Mouse Models of Huntington's Disease. J Neurosci. 2002; 22:1592-1599. [PubMed: 11880489]

1031. Møllersen L, Moldestad O, Rowe AD, Bjølgerud A, Holm I, Tveterås L, Klungland A, Retterstøl L. Effects of Anthocyanins on CAG Repeat Instability and Behaviour in Huntington's Disease R6/1 Mice. PLoS Curr. 2016; 8 ecurrents.hd. 58d04209ab04206d04205de00844db04207ef05628ff04267.

1032. Peyser C, Folstein M, Chase G, Starkstein S, Brandt J, Cockrell J, Bylsma F, Coyle J, Mchugh P, Folstein S. Trial of d-alpha-Tocopherol in Huntington's Disease. Am J Psychiatry. 1995; 152:1771-1775. [PubMed: 8526244]

1033. Mcgarry A, Mcdermott M, Kieburtz K, De Blieck EA, Beal F, Marder K, Ross C, Shoulson I, Gilbert P, Mallonee WM, et al. A Randomized, Double-Blind, Placebo-Controlled Trial of Coenzyme Q10 in Huntington Disease. Neurology. 2017; 88:152-159. [PubMed: 27913695]

1034. Gil-Mohapel J, Brocardo PS, Christie BR. The Role of Oxidative Stress in Huntington's Disease: Are Antioxidants Good Therapeutic Candidates? Curr Drug Targets. 2014; 15:454-468. [PubMed: 24428525]

1035. Machiela E, Dues DJ, Senchuk MM, Van Raamsdonk JM. Oxidative Stress is Increased in C. elegans Models of Huntington's Disease but Does Not Contribute to Polyglutamine Toxicity Phenotypes. Neurobiol Dis. 2016; 96:1-11. [PubMed: 27544481]

1036. Ito H, Wate R, Zhang J, Ohnishi S, Kaneko S, Ito H, Nakano S, Kusaka H. Treatment with Edaravone, Initiated at Symptom Onset, Slows Motor Decline and Decreases SOD1 Deposition in ALS Mice. Exp Neurol. 2008; 213:448-455. [PubMed: 18718468]

1037. Abe K, Itoyama Y, Sobue G, Tsuji S, Aoki M, Doyu M, Hamada C, Kondo K, Yoneoka T, Akimoto M, et al. Confirmatory Double-Blind, Parallel-Group, Placebo-Controlled Study of Efficacy And Safety of Edaravone (MCI-186) in Amyotrophic Lateral Sclerosis Patients. Amyotrophic Lateral Scler Frontotemporal Degener. 2014; 15:610-617.

1038. Wang B, Liu Q, Shan H, Xia C, Liu Z. Nrf2 Inducer and cncC Overexpression Attenuates Neurodegeneration due to a-Synuclein in Drosophila. Biochem Cell Biol. 2015; 93:351-358. [PubMed: 26008822]

1039. Stack C, Ho D, Wille E, Calingasan NY, Williams C, Liby K, Sporn M, Dumont M, Beal MF. Triterpenoids CDDO-Ethyl Amide and CDDO-Trifluoroethyl Amide Improve the Behavioral Phenotype and Brain Pathology in a Transgenic Mouse Model of Huntington's Disease. Free Radical Biol Med. 2010; 49:147-158. [PubMed: 20338236]

1040. Dinkova-Kostova AT, Kazantsev AG. Activation of Nrf2 Signaling as a Common Treatment of Neurodegenerative Diseases. Neurodegener Dis Manag. 2017; 7:97-100. [PubMed: 28534719]

1041. Di Domenico F, Tramutola A, Butterfield DA. Role of 4-Hydroxy-2-nonenal (HNE) in the Pathogenesis of Alzheimer Disease and Other Selected Age-Related Neurodegenerative Disorders. Free Radical Biol Med. 2017; 111:253-261. [PubMed: 27789292]

1042. Zhang S, Eitan E, Mattson MP. Early Involvement of Lysosome Dysfunction in the Degeneration of Cerebral Cortical Neurons Caused by the Lipid Peroxidation Product 4-Hydroxynonenal. J Neurochem. 2017; 140:941-954. [PubMed: 28095639] 
1043. Mark RJ, Lovell MA, Markesbery WR, Uchida K, Mattson MP. A Role for 4-Hydroxynonenal, an Aldehydic Product of Lipid Peroxidation, in Disruption of Ion Homeostasis and Neuronal Death Induced by Amyloid $\beta$-Peptide. J Neurochem. 1997; 68:255-264. [PubMed: 8978733]

1044. Kim H, Park BS, Lee KG, Choi CY, Jang SS, Kim YH, Lee SE. Effects of Naturally Occurring Compounds on Fibril Formation and Oxidative Stress of $\beta$-Amyloid. J Agric Food Chem. 2005; 53:8537-8541. [PubMed: 16248550]

1045. Maria SGA, Ignacio MMJ, Ramírez-Pineda Jose R, Marisol LR, Edison O, Patricia CGG. The Flavonoid Quercetin Ameliorates Alzheimer's Disease Pathology and Protects Cognitive and Emotional Function in Aged Triple Transgenic Alzheimer's Disease Model Mice. Neuropharmacology. 2015; 93:134-145. [PubMed: 25666032]

1046. Lee M, Mcgeer EG, Mcgeer PL. Quercetin, Not Caffeine, is a Major Neuroprotective Component in Coffee. Neurobiol Aging. 2016; 46:113-123. [PubMed: 27479153]

1047. Ansari MA, Abdul HM, Joshi G, Opii WO, Butterfield DA. Protective Effect of Quercetin in Primary Neurons Against A $\beta(1-42)$ : Relevance to Alzheimer's Disease. J Nutr Biochem. 2009; 20:269-275. [PubMed: 18602817]

1048. Iqbal N, Iqbal N. Imatinib: A Breakthrough of Targeted Therapy in Cancer. Chemother Res Pract. 2014; 2014:9.

1049. Kumar A, Kumar Singh S, Kumar V, Kumar D, Agarwal S, Rana MK. Huntington's Disease: An Update of Therapeutic Strategies. Gene. 2015; 556:91-97. [PubMed: 25447911]

1050. Chabrier PE, Auguet M. Pharmacological Properties of BN82451: a Novel Multitargeting Neuroprotective Agent. CNS Drug Rev. 2007; 13:317-332. [PubMed: 17894648]

1051. Li Z, Wang P, Yu Z, Cong Y, Sun H, Zhang J, Zhang J, Sun C, Zhang Y, Ju X. The Effect of Creatine and Coenzyme Q10 Combination Therapy on Mild Cognitive Impairment in Parkinson's Disease. Eur Neurol. 2015; 73:205-211. [PubMed: 25792086]

1052. Du Y. Patent, U S, Ed. Chemigen; USA: 2015.

1053. Kemp K, Redondo J, Hares K, Rice C, Scolding N, Wilkins A. Oxidative Injury in Multiple Sclerosis Cerebellar Grey Matter. Brain Res. 2016; 1642:452-460. [PubMed: 27086975]

1054. Newcombe J, Li H, Cuzner ML. Low Density Lipoprotein Uptake by Macrophages in Multiple Sclerosis Plaques: Implications for Pathogenesis. Neuropathol Appl Neurobiol. 1994; 20:152162. [PubMed: 7521019]

1055. Choi IY, Lee P, Adany P, Hughes AJ, Belliston S, Denney DR, Lynch SG. In Vivo Evidence of Oxidative Stress in Brains of Patients with Progressive Multiple Sclerosis. Mult Scler. 2017; 24:1029-1038. [PubMed: 28569645]

1056. Ohl K, Tenbrock K, Kipp M. Oxidative Stress in Multiple Sclerosis: Central and Peripheral Mode of Action. Exp Neurol. 2016; 277:58-67. [PubMed: 26626971]

1057. Elena F, Boris C, Galina K, Maria M, Vladimir S. Mitochondria-Targeted Antioxidants as a Prospective Therapeutic Strategy for Multiple Sclerosis. Curr Med Chem. 2017; 24:2086-2114. [PubMed: 28302008]

1058. Sempere AP, Berenguer-Ruiz L, Khabbaz E. Oral BG-12 in Multiple Sclerosis. New Engl J Med. 2013; 368:1652-1653.

1059. Schulze-Topphoff U, Varrin-Doyer M, Pekarek K, Spencer CM, Shetty A, Sagan SA, Cree BaC, Sobel RA, Wipke BT, Steinman L, et al. Dimethyl Fumarate Treatment Induces Adaptive and Innate Immune Modulation Independent of Nrf2. Proc Natl Acad Sci U S A. 2016; 113:47774782. [PubMed: 27078105]

1060. Kornberg MD, Bhargava P, Kim PM, Putluri V, Snowman AM, Putluri N, Calabresi PA, Snyder SH. Dimethyl Fumarate Targets GAPDH and Aerobic Glycolysis to Modulate Immunity. Science. 2018; doi: 10.1126/science.aan4665

1061. Blewett MM, Xie J, Zaro BW, Backus KM, Altman A, Teijaro JR, Cravatt BF. Chemical Proteomic Map of Dimethyl Fumarate-Sensitive Cysteines in Primary Human T Cells. Sci Signaling. 2016; 9:rs10-rs10.

1062. Leung L, Kwong M, Hou S, Lee C, Chan JY. Deficiency of the Nrf1 and Nrf2 Transcription Factors Results in Early Embryonic Lethality and Severe Oxidative Stress. J Biol Chem. 2003; 278:48021-48029. [PubMed: 12968018] 
1063. Zhao G, Liu Y, Fang J, Chen Y, Li H, Gao K. Dimethyl Fumarate Inhibits the Expression and Function of Hypoxia-Inducible Factor-1a (HIF-1a). Biochem Biophys Res Commun. 2014; 448:303-307. [PubMed: 24569076]

1064. Truong TH, Carroll KS. Redox Regulation of Protein Kinases. Crit Rev Biochem Mol Biol. 2013; 48:332-356. [PubMed: 23639002]

1065. Delmastro-Greenwood M, Freeman BA, Wendell SG. Redox-Dependent Anti-Inflammatory Signaling Actions of Unsaturated Fatty Acids. Annu Rev Physiol. 2014; 76:79-105. [PubMed: 24161076]

1066. Sawa T, Ihara H, Ida T, Fujii S, Nishida M, Akaike T. Formation, Signaling Functions, and Metabolisms of Nitrated Cyclic Nucleotide. Nitric Oxide. 2013; 34:10-18. [PubMed: 23632125]

1067. Higdon A, Diers Anne r, Oh Joo y, Landar A, Darley-Usmar Victor m. Cell Signalling by Reactive Lipid Species: New Concepts and Molecular Mechanisms. Biochem J. 2012; 442:453464. [PubMed: 22364280]

1068. Hallenbeck KK, Turner DM, Renslo AR, Arkin MR. Targeting Non-Catalytic Cysteine Residues Through Structure-Guided Drug Discovery. Curr Top Med Chem. 2017; 17:4-15. [PubMed: 27449257]

1069. Panieri E, Santoro MM. ROS Homeostasis and Metabolism: A Dangerous Liason in Cancer Cells. Cell Death Dis. 2016; 7:e2253. [PubMed: 27277675]

1070. Stratton MR, Campbell PJ, Futreal PA. The Cancer Genome. Nature. 2009; 458:719-724. [PubMed: 19360079]

1071. Chio IIC, Tuveson DA. ROS in Cancer: The Burning Question. Trends Mol Med. 2017; 23:411429. [PubMed: 28427863]

1072. Le Belle JE, Orozco NM, Paucar AA, Saxe JP, Mottahedeh J, Pyle AD, Wu H, Kornblum HI. Proliferative Neural Stem Cells Have High Endogenous ROS Levels that Regulate Self-Renewal and Neurogenesis in a PI3K/Akt-Dependent Manner. Cell Stem Cell. 2011; 8:59-71. [PubMed: 21211782]

1073. Williams CS, Mann M, Dubois RN. The Role of Cyclooxygenases in Inflammation, Cancer, and Development. Oncogene. 1999; 18:7908-7916. [PubMed: 10630643]

1074. Covarrubias L, Hernández-García D, Schnabel D, Salas-Vidal E, Castro-Obregón S. Function of Reactive Oxygen Species During Animal Development: Passive or Active? Dev Biol. 2008; 320:1-11. [PubMed: 18555213]

1075. Dennery PA. Effects of Oxidative Stress on Embryonic Development. Birth Defects Res Part C. 2007; 81:155-162.

1076. Ma Y, Zhang P, Wang F, Yang J, Yang Z, Qin H. The Relationship between Early Embryo Development and Tumourigenesis. J Cell Mol Med. 2010; 14:2697-2701. [PubMed: 21029369]

1077. Aiello NM, Stanger BZ. Echoes of the Embryo: Using the Developmental Biology Toolkit to Study Cancer. Dis Models \& Mech. 2016; 9:105-114.

1078. Loeb LA, Loeb KR, Anderson JP. Multiple Mutations and Cancer. Proc Natl Acad Sci U S A. 2003; 100:776-781. [PubMed: 12552134]

1079. Raj L, Ide T, Gurkar AU, Foley M, Schenone M, Li X, Tolliday NJ, Golub TR, Carr SA, Shamji AF, et al. Selective Killing of Cancer Cells by a Small Molecule Targeting the Stress Response to ROS. Nature. 2011; 475:231-234. [PubMed: 21753854]

1080. Ogrunc M, Di Micco R, Liontos M, Bombardelli L, Mione M, Fumagalli M, Gorgoulis VG, D'adda Di Fagagna F. Oncogene-Induced Reactive Oxygen Species Fuel Hyperproliferation and DNA Damage Response Activation. Cell Death Differ. 2014; 21:998-1012. [PubMed: 24583638]

1081. Delerue T, Khosrobakhsh F, Daloyau M, Emorine LJ, Dedieu A, Herbert CJ, Bonnefoy N, Arnauné-Pelloquin L, Belenguer P. Loss of Msp1p in Schizosaccharomyces pombe Induces a ROS-Dependent Nuclear Mutator Phenotype that Affects Mitochondrial Fission Genes. FEBS Lett. 2016; 590:3544-3558. [PubMed: 27664110]

1082. Halazonetis TD, Gorgoulis VG, Bartek J. An Oncogene-Induced DNA Damage Model for Cancer Development. Science. 2008; 319:1352-1355. [PubMed: 18323444]

1083. Zhang CZ, Leibowitz ML, Pellman D. Chromothripsis and Beyond: Rapid Genome Evolution from Complex Chromosomal Rearrangements. Genes Dev. 2013; 27:2513-2530. [PubMed: 24298051] 
1084. Jones S, Chen W-D, Parmigiani G, Diehl F, Beerenwinkel N, Antal T, Traulsen A, Nowak MA, Siegel C, Velculescu VE, et al. Comparative Lesion Sequencing Provides Insights into Tumor Evolution. Proc Natl Acad Sci U S A. 2008; 105:4283-4288. [PubMed: 18337506]

1085. Bartesaghi S, Graziano V, Galavotti S, Henriquez NV, Betts J, Saxena J, Minieri VAD, Karlsson A, Martins LM, et al. Inhibition of Oxidative Metabolism Leads to p53 Genetic Inactivation and Transformation in Neural Stem Cells. Proc Natl Acad Sci U S A. 2015; 112:1059-1064. [PubMed: 25583481]

1086. Kruiswijk F, Labuschagne CF, Vousden KH. p53 in Survival, Death and Metabolic Health: A Lifeguard with a Licence to Kill. Nat Rev Mol Cell Biol. 2015; 16:393-405. [PubMed: 26122615]

1087. Gorrini C, Baniasadi PS, Harris IS, Silvester J, Inoue S, Snow B, Joshi PA, Wakeham A, Molyneux SD, Martin B, et al. BRCA1 Interacts with Nrf2 to Regulate Antioxidant Signaling and Cell Survival. J Exp Med. 2013; 210:1529-1544. [PubMed: 23857982]

1088. Funes JM, Henderson S, Kaufman R, Flanagan JM, Robson M, Pedley B, Moncada S, Boshoff C. Oncogenic Transformation of Mesenchymal Stem Cells Decreases Nrf2 Expression Favoring in Vivo Tumor Growth and Poorer Survival. Mol Cancer. 2014; 13:20. [PubMed: 24491031]

1089. Reczek CR, Chandel NS. The Two Faces of Reactive Oxygen Species in Cancer. Annu Rev Cancer Biol. 2017; 1:79-98.

1090. Ouyang WC, Liao YW, Chen PN, Lu KH, Yu CC, Hsieh PL. Hinokitiol Suppresses Cancer Stemness and Oncogenicity in Glioma Stem Cells by Nrf2 Regulation. Cancer Chemother Pharmacol. 2017; 80:411-419. [PubMed: 28685346]

1091. Kwak MK, Ryoo IG, Lee SH. Activation of NRF2 Signaling in Breast Cancer Stem CellEnriched Population. Free Radical Biol Med. 2016; 100:S125.

1092. Mayerhofer M, Florian S, Krauth M-T, Aichberger KJ, Bilban M, Marculescu R, Printz D, Fritsch G, Wagner O, Selzer E, et al. Identification of Heme Oxygenase-1 as a Novel Bcr/AblDependent Survival Factor in Chronic Myeloid Leukemia. Cancer Res. 2004; 64:3148-3154. [PubMed: 15126353]

1093. Yamadori T, Ishii Y, Homma S, Morishima Y, Kurishima K, Itoh K, Yamamoto M, Minami Y, Noguchi M, Hizawa N. Molecular Mechanisms for the Regulation of Nrf2-Mediated Cell Proliferation in Non-Small-Cell Lung Cancers. Oncogene. 2012; 31:4768-4777. [PubMed: 22249257]

1094. Greaves M, Maley CC. Clonal Evolution in Cancer. Nature. 2012; 481:306-313. [PubMed: 22258609]

1095. Chung FL, Chen HJC, Guttenplan JB, Nishikawa A, Hard GC. 2,3-Epoxy-4-Hydroxynonanal as a Potential Tumor-Initiating Agent of Lipid Peroxidation. Carcinogenesis. 1993; 14:2073-2077. [PubMed: 8222056]

1096. Eckl PM, Bresgen N. Genotoxicity of Lipid Oxidation Compounds. Free Radical Biol Med. 2017; 111:244-252. [PubMed: 28167130]

1097. Hu W, Feng Z, Eveleigh J, Iyer G, Pan J, Amin S, Chung FL, Tang MS. The Major Lipid Peroxidation Product, Trans- 4-Hydroxy-2-nonenal, Preferentially Forms DNA Adducts at Codon 249 of Human P53 Gene, a Unique Mutational Hotspot in Hepatocellular Carcinoma. Carcinogenesis. 2002; 23:1781-1789. [PubMed: 12419825]

1098. Bekerecioğlu M, Aslan R, Uğras S, Kutluhan A, Sekeroğlu R, Akpolat N, Karakok M. Malondialdehyde Levels in Serum of Patients with Skin Cancer. Eur J Plast Surg. 1998; 21:227229.

1099. Juric-Sekhar G, Zarkovic K, Waeg G, Cipak A, Zarkovic N. Distribution of 4-HydroxynonenalProtein Conjugates as a Marker of Lipid Peroxidation and Parameter of Malignancy in Astrocytic and Ependymal Tumors of the Brain. Tumori. 2015; 95:762-768.

1100. Gęgotek A, Nikliński J, Žarković N, Žarković K, Waeg G, Łuczaj W, Charkiewicz R, Skrzydlewska E. Lipid Mediators Involved in the Oxidative Stress and Antioxidant Defence of Human Lung Cancer Cells. Redox Biol. 2016; 9:210-219. [PubMed: 27567474]

1101. Cheeseman KH. Mechanisms and Effects of Lipid Peroxidation. Mol Aspects Med. 1993; 14:191-197. [PubMed: 8264333] 
1102. Dianzani MU. Lipid Peroxidation and Cancer. Crit Rev Oncol Hematol. 1993; 15:125-147. [PubMed: 8117415]

1103. Hammer A, Ferro M, Tillian HM, Tatzber F, Zollner H, Schauenstein E, Schaur RJ. Effect of Oxidative Stress by Iron on 4-Hydroxynonenal Formation and Proliferative Activity in Hepatomas of Different Degrees of Differentiation. Free Radical Biol Med. 1997; 23:26-33. [PubMed: 9165294]

1104. Barrera G. Oxidative Stress and Lipid Peroxidation Products in Cancer Progression and Therapy. ISRN Oncol. 2012; 2012:21.

1105. Zarkovic N, Ilic Z, Jurin M, Schaur RJ, Puhl H, Esterbauer H. Stimulation of HeLa Cell Growth by Physiological Concentrations of 4-Hydroxynonenal. Cell Biochem Funct. 1993; 11:279-286. [PubMed: 8275553]

1106. Barrera G, Martinotti S, Fazio V, Manzari V, Paradisi L, Parola M, Frati L, Dianzani MU. Effect of 4-Hydroxynonenal on c-myc Expression. Toxicol Pathol. 1987; 15:238-240. [PubMed: 3475758]

1107. Cerbone A, Toaldo C, Laurora S, Briatore F, Pizzimenti S, Dianzani MU, Ferretti C, Barrera G. 4-Hydroxynonenal and PPAR $\gamma$ Ligands Affect Proliferation, Differentiation, and Apoptosis in Colon Cancer Cells. Free Radical Biol Med. 2007; 42:1661-1670. [PubMed: 17462534]

1108. Barrera G, Muraca R, Pizzimenti S, Serra A, Rosso C, Saglio G, Farace MG, Fazio VM, Dianzani MU. Inhibition of c-myc Expression Induced by 4-Hydroxynonenal, a Product of Lipid Peroxidation, in the HL-60 Human Leukemic Cell Line. Biochem Biophys Res Commun. 1994; 203:553-561. [PubMed: 8074703]

1109. Kakishita H, Hattori Y. Vascular Smooth Muscle Cell Activation and Growth by 4Hydroxynonenal. Life Sci. 2001; 69:689-697. [PubMed: 11476190]

1110. Ruef J, Rao GN, Li F, Bode C, Patterson C, Bhatnagar A, Runge MS. Induction of Rat Aortic Smooth Muscle Cell Growth by the Lipid Peroxidation Product 4-Hydroxy-2-Nonenal. Circulation. 1998; 97:1071-1078. [PubMed: 9531254]

1111. Muzio G, Ricci M, Traverso N, Monacelli F, Oraldi M, Maggiora M, Canuto RA. 4Hydroxyhexenal and 4-Hydroxynonenal Are Mediators of the Anti-Cachectic Effect of N-3 and N-6 Polyunsaturated Fatty Acids on Human Lung Cancer Cells. Free Radical Biol Med. 2016; 99:63-70. [PubMed: 27480845]

1112. Sharma R, Brown D, Awasthi S, Yang Y, Sharma A, Patrick B, Saini MK, Singh SP, Zimniak P, Singh SV, et al. Transfection with 4-Hydroxynonenal-Metabolizing Glutathione S-Transferase Isozymes Leads to Phenotypic Transformation and Immortalization of Adherent Cells. Eur J Biochem. 2004; 271:1690-1701. [PubMed: 15096208]

1113. Sharma R, Sharma A, Chaudhary P, Sahu M, Jaiswal S, Awasthi S, Awasthi YC. Role of 4Hydroxynonenal in Chemopreventive Activities of Sulforaphane. Free Radical Biol Med. 2012; 52:2177-2185. [PubMed: 22579574]

1114. Schnelldorfer T, Gansauge S, Gansauge F, Schlosser S, Beger HG, Nussler AK. Glutathione Depletion Causes Cell Growth Inhibition and Enhanced Apoptosis in Pancreatic Cancer Cells. Cancer. 2000; 89:1440-1447. [PubMed: 11013356]

1115. Singhal SS, Singh SP, Singhal P, Horne D, Singhal J, Awasthi S. Antioxidant Role of Glutathione S-Transferases: 4-Hydroxynonenal, a Key Molecule in Stress-Mediated Signaling. Toxicol Appl Pharmacol. 2015; 289:361-370. [PubMed: 26476300]

1116. Fazio VM, Rinaldi M, Ciafrè S, Barrera G, Farace MG. Control of Neoplastic Cell Proliferation and Differentiation by Restoration of 4-Hydroxynonenal Physiological Concentrations. Mol Aspects Med. 1993; 14:217-228. [PubMed: 8264336]

1117. Ollila S, Hyvönen MT, Vattulainen I. Polyunsaturation in Lipid Membranes:wDynamic Properties and Lateral Pressure Profiles. J Phys Chem B. 2007; 111:3139-3150. [PubMed: 17388448]

1118. Rysman E, Brusselmans K, Scheys K, Timmermans L, Derua R, Munck S, Van Veldhoven PP, Waltregny D, Daniëls VW, Machiels J, et al. De Novo Lipogenesis Protects Cancer Cells from Free Radicals and Chemotherapeutics by Promoting Membrane Lipid Saturation. Cancer Res. 2010; 70:8117-8126. [PubMed: 20876798] 
1119. Guillaumond F, Bidaut G, Ouaissi M, Servais S, Gouirand V, Olivares O, Lac S, Borge L, Roques J, Gayet O, et al. Cholesterol Uptake Disruption, in Association with Chemotherapy, is a Promising Combined Metabolic Therapy for Pancreatic Adenocarcinoma. Proc Natl Acad Sci U S A. 2015; 112:2473-2478. [PubMed: 25675507]

1120. Beloribi-Djefaflia S, Vasseur S, Guillaumond F. Lipid Metabolic Reprogramming in Cancer Cells. Oncogenesis. 2016; 5:e189. [PubMed: 26807644]

1121. Dequanter D, Dok R, Nuyts S. Basal Oxidative Stress Ratio of Head and Neck Squamous Cell Carcinomas Correlates with Nodal Metastatic Spread in Patients Under Therapy. OncoTargets Ther. 2017; 10:259-263.

1122. Krock BL, Skuli N, Simon MC. Hypoxia-Induced Angiogenesis: Good and Evil. Genes Cancer. 2011; 2:1117-1133. [PubMed: 22866203]

1123. Borutaite V, Brown GC. Caspases are Reversibly Inactivated by Hydrogen Peroxide. FEBS Lett. 2001; 500:114-118. [PubMed: 11445067]

1124. Tong L, Chuang CC, Wu S, Zuo L. Reactive Oxygen Species in Redox Cancer Therapy. Cancer Lett. 2015; 367:18-25. [PubMed: 26187782]

1125. Nishikawa M. Reactive Oxygen Species in Tumor Metastasis. Cancer Lett. 2008; 266:53-59. [PubMed: 18362051]

1126. Nagel R, Semenova EA, Berns A. Drugging the Addict: Non-Oncogene Addiction as a Target for Cancer Therapy. EMBO Rep. 2016; 17:1516-1531. [PubMed: 27702988]

1127. Trachootham D, Zhou Y, Zhang H, Demizu Y, Chen Z, Pelicano H, Chiao PJ, Achanta G, Arlinghaus RB, Liu J, et al. Selective Killing of Oncogenically Transformed Cells Through a ROS-Mediated Mechanism by Beta-Phenylethyl Isothiocyanate. Cancer Cell. 2006; 10:241-252. [PubMed: 16959615]

1128. Zhao Y, Miriyala S, Miao L, Mitov M, Schnell D, Dhar SK, Cai J, Klein JB, Sultana R, Butterfield DA, et al. Redox Proteomic Identification Of HNE-Bound Mitochondrial Proteins in Cardiac Tissues Reveals a Systemic Effect on Energy Metabolism after Doxorubicin Treatment. Free Radical Biol Med. 2014; 72:55-65. [PubMed: 24632380]

1129. Li Y, Tian F, Shi P, Guo L, Wu H, Chen R, Xue J. 4-Hydroxynonenal Promotes Growth and Angiogenesis of Breast Cancer Cells through HIF-1a Stabilization. Asian Pac J Cancer Prev. 2014; 15:10151-10156. [PubMed: 25556440]

1130. Wang H, Linetsky M, Guo J, Choi J, Hong L, Chamberlain AS, Howell SJ, Howes AM, Salomon RG. 4-Hydroxy-7-oxo-5-heptenoic Acid (HOHA) Lactone is a Biologically Active Precursor for the Generation of 2-( $\omega$-Carboxyethyl)pyrrole (CEP) Derivatives of Proteins and Ethanolamine Phospholipids. Chem Res Toxicol. 2015; 28:967-977. [PubMed: 25793308]

1131. Singhal SS, Singhal J, Yadav S, Sahu M, Awasthi YC, Awasthi S. RLIP76: A Target for Kidney Cancer Therapy. Cancer Res. 2009; 69:4244-4251. [PubMed: 19417134]

1132. Probin V, Wang Y, Zhou D. Busulfan-Induced Senescence is Dependent upon ROS Production Upstream of The MAPK Pathway. Free Radical Biol Med. 2007; 42:1858-1865. [PubMed: 17512465]

1133. Wang X, Mazurkiewicz M, Hillert EK, Olofsson MH, Pierrou S, Hillertz P, Gullbo J, Selvaraju K, Paulus A, Akhtar S, et al. The Proteasome Deubiquitinase Inhibitor VLX1570 Shows Selectivity for Ubiquitin-Specific Protease-14 and Induces Apoptosis of Multiple Myeloma Cells. Sci Rep. 2016; 6:26979. [PubMed: 27264969]

1134. Booth L, Malkin M, Dent P. Repurposing Tecfidera for Cancer. Aging (N Y). 2016; 8:1289_ 1290.

1135. Kastrati I, Siklos MI, Calderon-Gierszal EL, El-Shennawy L, Georgieva G, Thayer EN, Thatcher GRJ, Frasor J. Dimethyl Fumarate Inhibits the Nuclear Factor $x b$ Pathway in Breast Cancer Cells by Covalent Modification of p65 Protein. J Biol Chem. 2016; 291:3639-3647. [PubMed: 26683377]

1136. Pohl SG, Brook N, Agostino M, Arfuso F, Kumar AP, Dharmarajan A. Wnt Signaling in TripleNegative Breast Cancer. Oncogenesis. 2017; 6:e310. [PubMed: 28368389]

1137. Rada P, Rojo AI, Offergeld A, Feng GJ, Velasco-Martín JP, González-Sancho JM, Valverde ÁM, Dale T, Regadera J, Cuadrado A. WNT-3A Regulates an Axin1/Nrf2 Complex that Regulates 
Antioxidant Metabolism in Hepatocytes. Antioxid Redox Signal. 2015; 22:555-571. [PubMed: 25336178]

1138. Manigandan K, Manimaran D, Jayaraj RL, Elangovan N, Dhivya V, Kaphle A. Taxifolin Curbs NF- $\kappa$ B-Mediated Wnt/ $\beta$-Catenin Signaling via Up-Regulating Nrf2 Pathway in Experimental Colon Carcinogenesis. Biochimie. 2015; 119:103-112. [PubMed: 26482805]

1139. Huo L, Li C-W, Huang T-H, Lam YC, Xia W, Tu C, Chang W-C, Hsu JL, Lee D-F, Nie L, et al. Activation of Keap1/Nrf2 Signaling Pathway by Nuclear Epidermal Growth Factor Receptor in Cancer Cells. Am J Transl Res. 2014; 6:649-663. [PubMed: 25628777]

1140. Papaiahgari S, Zhang Q, Kleeberger SR, Cho HY, Reddy SP. Hyperoxia Stimulates an Nrf2ARE Transcriptional Response via ROS-EGFR-PI3K-Akt/ERK MAP Kinase Signaling in Pulmonary Epithelial Cells. Antioxid Redox Signal. 2006; 8:43-52. [PubMed: 16487036]

1141. Liu W, Mao L, Ji F, Chen F, Wang S, Xie Y. Icariside II Activates EGFR-Akt-Nrf2 Signaling and Protects Osteoblasts from Dexamethasone. Oncotarget. 2017; 8:2594-2603. [PubMed: 27911877]

1142. Wakabayashi N, Shin S, Slocum SL, Agoston ES, Wakabayashi J, Kwak M-K, Misra V, Biswal S, Yamamoto M, Kensler TW. Regulation of Notch1 Signaling by Nrf2: Implications for Tissue Regeneration. Sci Signaling. 2010; 3:ra52-ra52.

1143. Purow BW, Sundaresan TK, Burdick MJ, Kefas BA, Comeau LD, Hawkinson MP, Su Q, Kotliarov Y, Lee J, Zhang W, et al. Notch-1 Regulates Transcription of the Epidermal Growth Factor Receptor Through p53. Carcinogenesis. 2008; 29:918-925. [PubMed: 18359760]

1144. Reddy NM, Potteti HR, Vegiraju S, Chen HJ, Tamatam CM, Reddy SP. PI3K-AKT Signaling via Nrf2 Protects Against Hyperoxia-Induced Acute Lung Injury, but Promotes Inflammation PostInjury Independent of Nrf2 in Mice. PLoS One. 2015; 10:e0129676. [PubMed: 26075390]

1145. Abazeed ME, Adams DJ, Hurov KE, Tamayo P, Creighton CJ, Sonkin D, Giacomelli AO, Du C, Fries DF, Wong K-K, et al. Integrative Radiogenomic Profiling of Squamous Cell Lung Cancer. Cancer Res. 2013; 73:6289-6298. [PubMed: 23980093]

1146. Meitzler JL, Antony S, Wu Y, Juhasz A, Liu H, Jiang G, Lu J, Roy K, Doroshow JH. NADPH Oxidases: A Perspective on Reactive Oxygen Species Production in Tumor Biology. Antioxid Redox Signal. 2014; 20:2873-2889. [PubMed: 24156355]

1147. Banskota S, Regmi SC, Kim JA. NOX1 to NOX2 Switch Deactivates AMPK and Induces Invasive Phenotype in Colon Cancer Cells Through Overexpression of MMP-7. Mol Cancer. 2015; 14:123. [PubMed: 26116564]

1148. Steinhubl SR. Why Have Antioxidants Failed in Clinical Trials? Am J Cardiol. 2008; 101:S14S19.

1149. Singh J, Petter RC, Baillie TA, Whitty A. The Resurgence of Covalent Drugs. Nat Rev Drug Discov. 2011; 10:307-317. [PubMed: 21455239]

1150. Wenjuan Z, Yaofei B, Yonghua W, Wei X. Polypharmacology in Drug Discovery: A Review from Systems Pharmacology Perspective. Curr Pharm Des. 2016; 22:3171-3181. [PubMed: 26907941]

1151. Bauer RA. Covalent Inhibitors in Drug Discovery: From Accidental Discoveries to Avoided Liabilities and Designed Therapies. Drug Discovery Today. 2015; 20:1061-1073. [PubMed: 26002380]

1152. Johnson DS, Weerapana E, Cravatt BF. Strategies for Discovering and Derisking Covalent, Irreversible Enzyme Inhibitors. Future Med Chem. 2010; 2:949-964. [PubMed: 20640225]

1153. Liu Q, Sabnis Y, Zhao Z, Zhang T, Buhrlage SJ, Jones LH, Gray NS. Developing Irreversible Inhibitors of the Protein Kinase Cysteinome. Chem Biol. 2013; 20:146-159. [PubMed: 23438744]

1154. Knight ZA, Lin H, Shokat KM. Targeting the Cancer Kinome Through Polypharmacology. Nat Rev Cancer. 2010; 10:130-137. [PubMed: 20094047]

\section{Biographies}

Saba Parvez 
Saba Parvez was born and raised in India. He received his undergraduate training in biochemistry at Bates College, USA where he worked with Prof. Rachel Austin. He then moved to Ithaca to undertake his doctoral studies under the guidance of Professor Yimon Aye as an HHMI international predoctoral fellow. He is interested in studying cell signaling pathways, and understand how the deregulation of these pathways cause disease.

\section{Marcus Long.}

Marcus Long was born and raised in England. He read chemistry at Oxford University, UK. Having completed his Ph.D. in biochemistry in Brandeis University, USA, as an HHMI international predoctoral fellow under the guidance of Professor Lizbeth Hedstrom, he now focuses on understanding the small-molecule regulation of cell signaling pathways, including redox signaling in the Aye laboratory.

Jesse Poganik:

Jesse Poganik was born and raised in Queens, New York. He received his B.S. in Chemistry from the State University of New York at Stony Brook where he worked in the lab of Prof. Dale Drueckhammer. He then went on to pursue doctoral studies in chemical biology in the lab of Prof. Yimon Aye at Cornell University in Ithaca, New York. His ongoing thesis research as an American Heart Association predoctoral fellow focuses on understanding novel regulatory pathways controlled by precision signaling activities of reactive electrophiles.

Yimon Aye:

Yimon Aye was born and raised in Burma. She read chemistry at Oxford University, UK, and subsequently completed her graduate training in organic chemistry with Professor David Evans at Harvard University, USA, in 2009. She then pursued her postdoctoral training with Professor JoAnne Stubbe at Massachusetts Institute of Technology, researching the cellular and biochemical regulatory mechanisms of the enzyme ribonucleotide reductase. In her independent career at Cornell University that began in mid-2012, she set out to understand the detailed mechanisms of precision electrophile signaling. This impetus culminated in the development of REX technologies, T-REX ${ }^{\mathrm{TM}}$ delivery and G-REX ${ }^{\mathrm{TM}}$ profiling. 


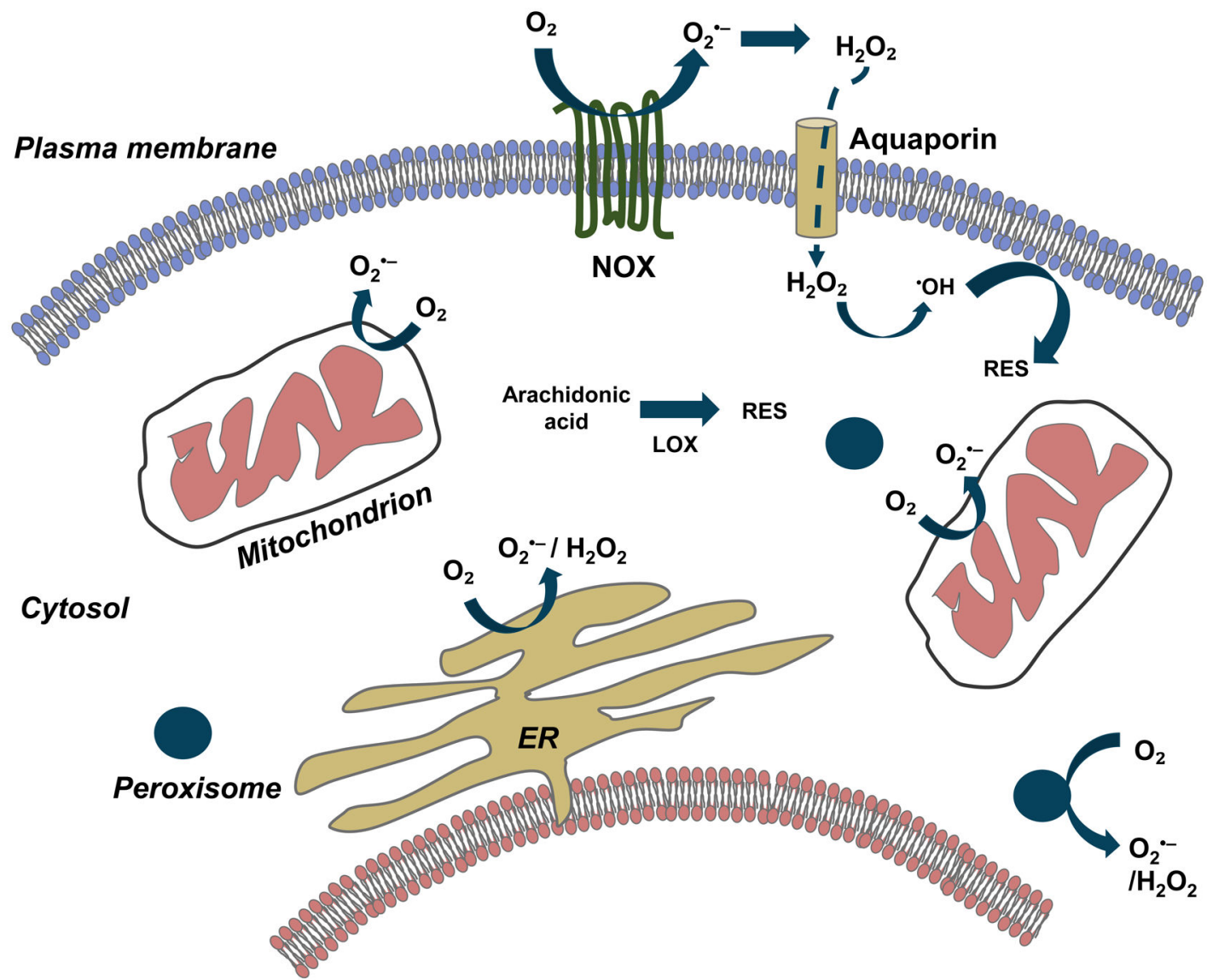

Nucleus

Figure 1.

The compartmentalized/subcellular generation of various chemotypes of ROS and RES. Some of the most important sources of ROS in biological systems include NOX enzymes localized on plasma membrane and membranes of cellular organelles, mitochondrial ETC, and metabolic enzymes in the ER and peroxisomes. Enzymatic and non-enzymatic oxidation of fatty acids are the primary sources of RES in mammalian cells. 

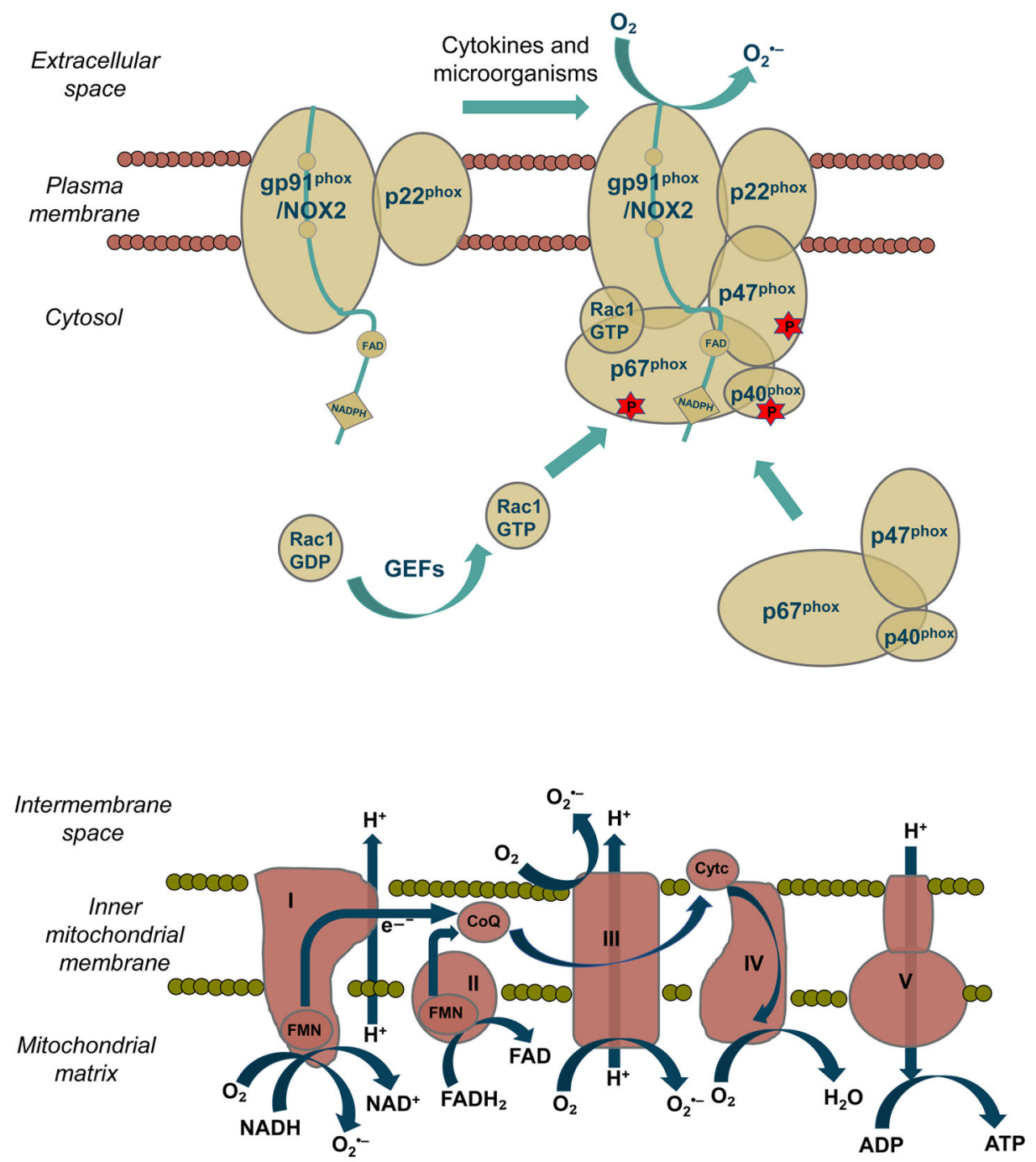


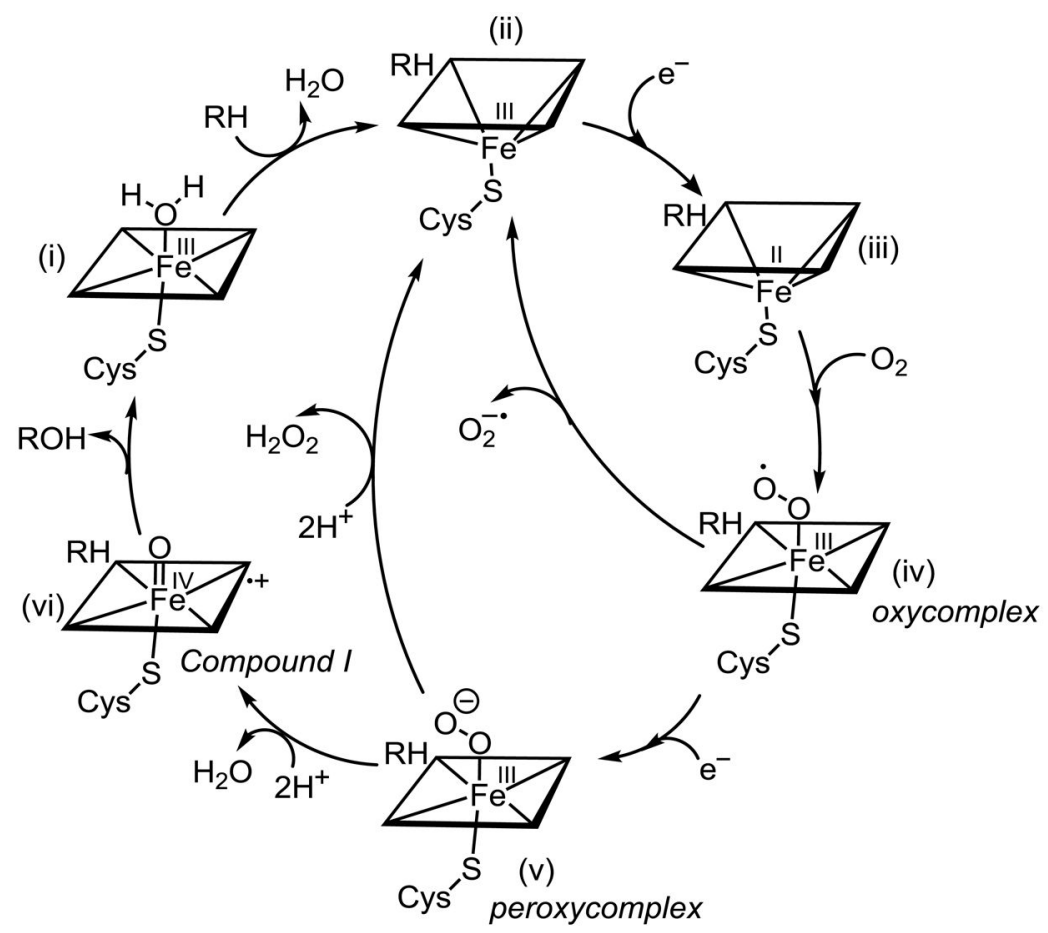

Figure 2.

Sites of ROS generation in eukaryotic cells (A) NOX enzymes generate $\mathrm{O}_{2}{ }^{-}$by a single electron reduction of ${ }^{3} \mathrm{O}_{2}$. $(144,145)$ NOX enzymes (NOX2 shown here), consist of multisubunit complexes comprising transmembrane subunits (gp91 ${ }^{\text {phox }} / \mathrm{NOX} 2$ and $\mathrm{p} 22^{\text {phox }}$ ) and various cytosolic regulatory domains. Stimulation with cytokines or microorganisms results in phosphorylation and subsequent recruitment of the cytosolic components p67phox, $\mathrm{p} 47^{\text {phox }}$, and $\mathrm{p} 40^{\text {phox }}$ and a Rac1 GTP to the transmembrane subunits. The p67 ${ }^{\text {phox }}$ initiates electron transfer by accepting two electrons from NADPH. The electrons are relayed through FAD and then sequentially to two heme cofactors (depicted as orange circles) housed in the transmembrane gp91 ${ }^{\text {phox }}$ domain. $\mathrm{O}_{2}$ acts as the terminal electron acceptor generating two molecules of $\mathrm{O}_{2}{ }^{-}$per molecule of NADPH used. GDI: guanosine nucleotide dissociation inhibitor. (B) Complex I and Complex III are the two important sites of $\mathrm{O}_{2}$ ${ }^{-}$-generation in the mitochondrial electron transport chain (mETC). At Complex I, $\mathrm{O}_{2}{ }^{-}$is generated by the reaction of ${ }^{3} \mathrm{O}_{2}$ with reduced FMN. $\mathrm{O}_{2}{ }^{-}$-generation at Complex I is favored under conditions of high proton motive force and when $\mathrm{CoQ}$ is reduced, resulting in a reverse electron transfer from reduced $\mathrm{CoQ}$ to FMN site at Complex I. ${ }^{3} \mathrm{O}_{2}$ reduction at Complex III is mediated by ubisemiquinone housed in this subunit. Complex I releases $\mathrm{O}_{2}$ -- primarily in the mitochondrial matrix whereas Complex III generated $\mathrm{O}_{2}{ }^{-}{ }^{-}$is released in both the mitochondrial matrix and the intermembrane space. (C) The catalytic cycle of microsomal monooxygenase (MMOs) CYP450. CYP450 is a heme-iron-containing protein. In the resting state, iron is in a hexa-coordinated ferric form $\left(\mathrm{Fe}^{3+}\right)$-equatorial sites are taken up by the heme cofactor (denoted by rhombus); a cysteine thiol from the protein, and a water molecule occupy the apical sites (i). Substrate binding displaces the water molecule, and a subsequent one-electron reduction by NAD(P)H via FAD/FMN-containing CYP450 reductase (CPR) generates the penta-coordinated ferrous complex $\left(\mathrm{Fe}^{2+}\right)$ (iii). Addition of 
$\mathrm{O}_{2}$ to (iii) generates a hexa-coordinated $\mathrm{Fe}^{3+-} \mathrm{O}_{2}$ oxycomplex (iv). MMOs in the eukaryotic ER generate ROS because of inefficient utilization of activated $\mathrm{O}_{2}$ for substrate oxidation. Two uncoupling reactions that result in the generation of ROS are shown. The first route generates $\mathrm{O}_{2}{ }^{-}$-due to the decay of one-electron reduced oxycomplex (iv). A possible route of $\mathrm{H}_{2} \mathrm{O}_{2}$ generation includes protonation and subsequent uncoupling of the peroxycomplex (v). Adapted with permission from ref. (146). Copyright 2004 Elsevier. 


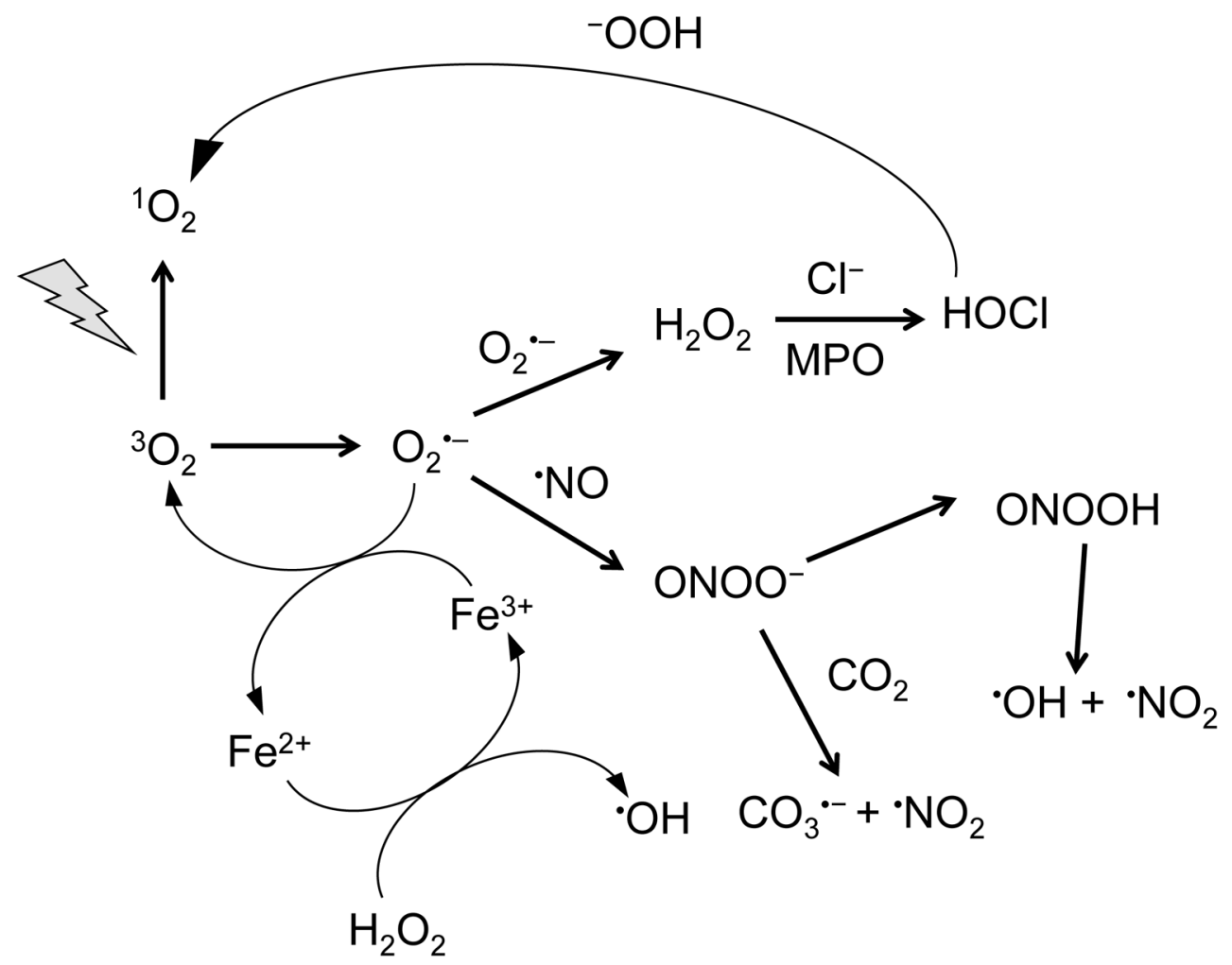

Figure 3.

ROS interconversion in eukaryotic cells. ROS generated in cells are highly dynamic and rapidly interconvert. $\mathrm{O}_{2}{ }^{-}$- generated by enzymatic pathways such as NOX enzymes and mETC by reduction of ground-state ${ }^{3} \mathrm{O}_{2}$, rapidly dismutates to yield $\mathrm{H}_{2} \mathrm{O}_{2}$. SODs also catalyze this conversion. $\mathrm{O}_{2}{ }^{-}$- also reacts with ${ }^{\circ} \mathrm{NO}$ yielding $\mathrm{ONOO}^{-}$, which can further decompose to generate ${ }^{\circ} \mathrm{OH} . \mathrm{H}_{2} \mathrm{O}_{2}$ generates ${ }^{\circ} \mathrm{OH}$ in presence of trace metals such as reduced iron. Reactive radicals such as ${ }^{\circ} \mathrm{OH}$ initiate lipid peroxidation by abstracting a bisallylic hydrogen from PUFAs (see Figure 5a). Myeloperoxidase enzyme (MPO) in neutrophils and macrophages(203) utilize $\mathrm{H}_{2} \mathrm{O}_{2}$ to generate $\mathrm{HOCl}$ that can react with ${ }^{-} \mathrm{OOH}$ to generate singlet oxygen $\left({ }^{1} \mathrm{O}_{2}\right)$ in phagocytic cells. ${ }^{1} \mathrm{O}_{2}$ is also directly generated by the excitation of ${ }^{3} \mathrm{O}_{2}$ by $\mathrm{UV}$ radiation. 

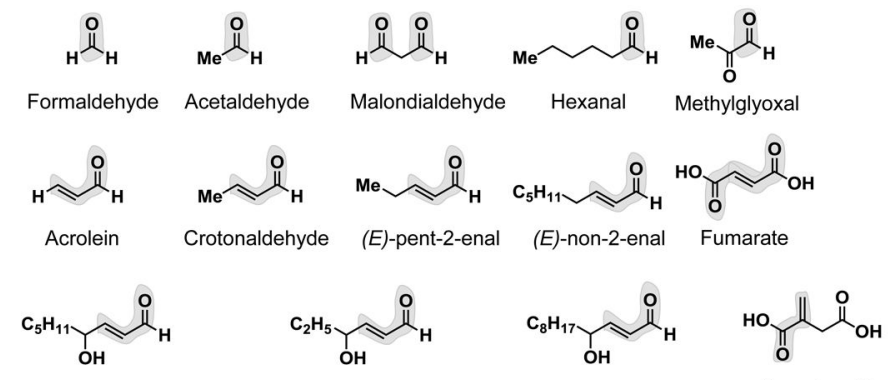

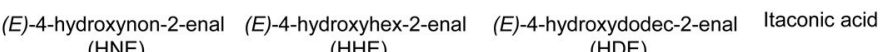
(HNE) (HDE)

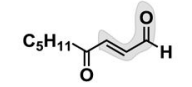

(E)-4-oxonon-2-enal (ONE)

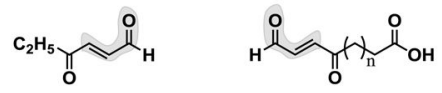
$\begin{array}{ll}\text { (E)-4-oxohex-2-enal } & \mathrm{n}=6: 9,12 \text { dioxo-10dodecenoic acid } \\ \mathrm{n}=2: 5,8 \text { dioxo-10octenoic acid }\end{array}$

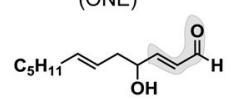

4-hydroxy-2,6-dodecadienal $\quad 4,5$ epoxy-2-(E)decenal $\quad(2 E, 4 E)$-deca-2,4-dienal L-DOPAquinone
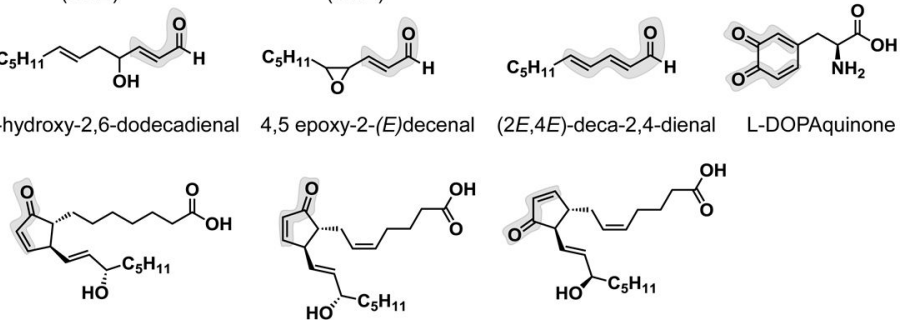

Prostaglandin A1 $\left(\mathrm{PGA}_{1}\right)$

Prostaglandin A2 $\left(\mathrm{PGA}_{2}\right)$ Prostaglandin J2 $\left(\mathrm{PGJ}_{2}\right)$
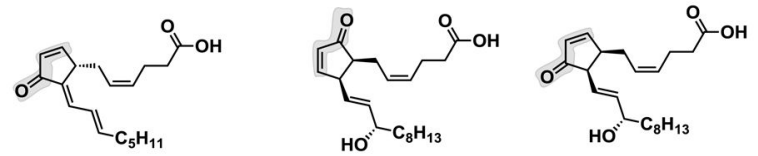

15-deoxy- $\triangle$-12,14-prostaglandin J2 $\left(15 \mathrm{~d}-\mathrm{PGJ}_{2}\right)$

$\mathrm{A}_{4}$-Neuroprostane

$\mathrm{J}_{4}$-Neuroprostane
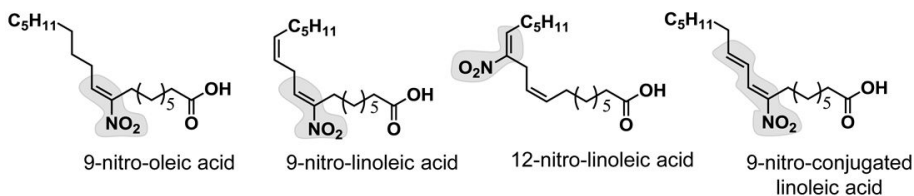

Figure 4.

A selection of endogenously-generated carbonyl-containing reactive electrophilic species (RES) in biological systems. 


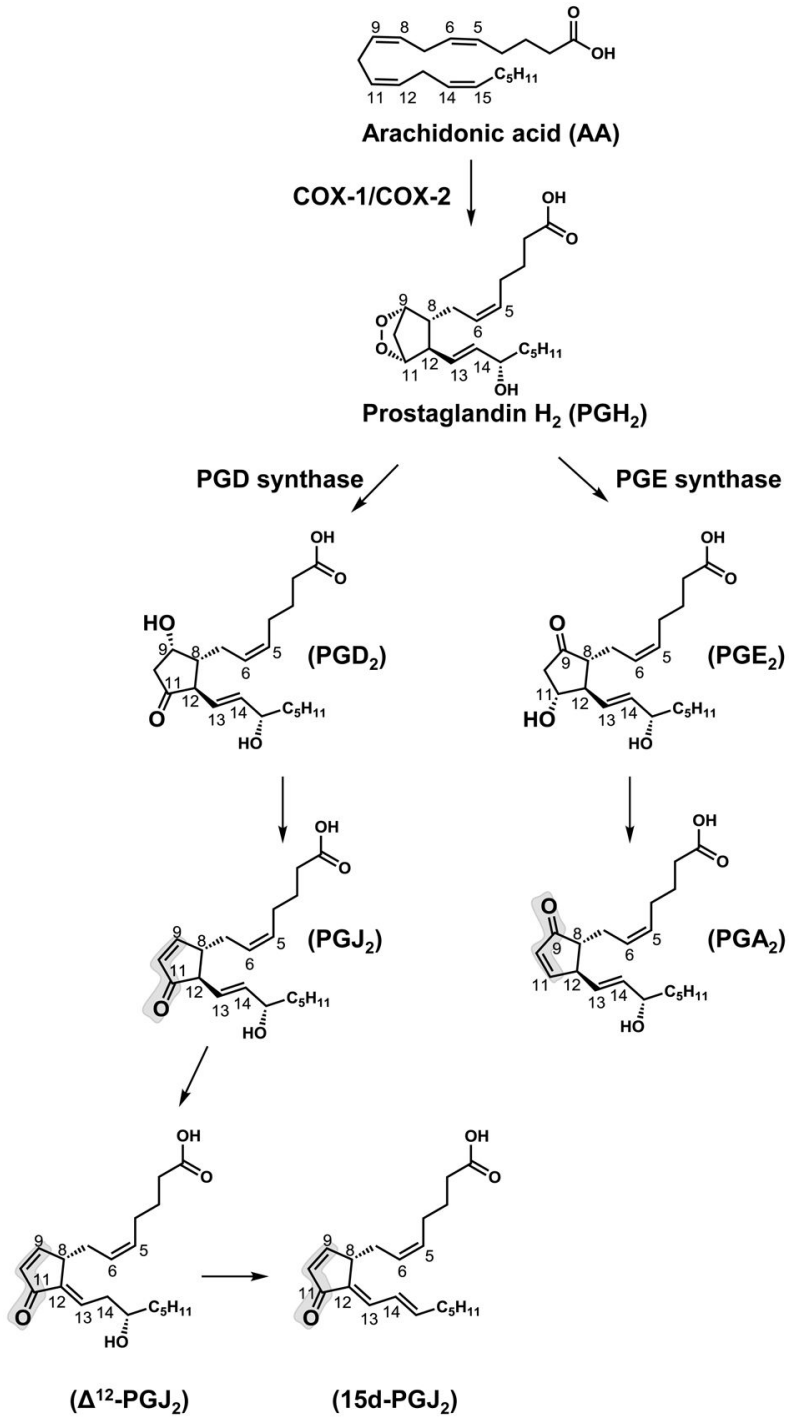

Figure 5.

Enzymatic generation of lipid-derived RES.(250) COX-1/COX-2-catalyzes the formation of $\mathrm{PGH}_{2}$ using arachidonic acid (AA) as the substrate. $\mathrm{PGH}_{2}$ acts as an intermediate for the generation of $\mathrm{PGD}_{2}$ and $\mathrm{PGE}_{2}$ by the action of PGD synthase and PGE synthase,

respectively. $\mathrm{PGH}_{2}$ is also an intermediate for the generation of a number of other bioactive non-electrophilic molecules (not shown). $\mathrm{PGD}_{2}$ and $\mathrm{PGE}_{2}$ undergo spontaneous dehydration at $\mathrm{C}_{(9)}$ and $\mathrm{C}_{(11)}$ to yield $\mathrm{PGJ}_{2}$ and $\mathrm{PGA}_{2}$, respectively. $\mathrm{PGJ}_{2}$ can then isomerize at $\mathrm{C}_{(12)}-$ $\mathrm{C}_{(13)}-\mathrm{C}_{(14)}$ to generate $\Delta^{12}-\mathrm{PGJ}_{2}$ and further dehydrate at $\mathrm{C}_{(15)}$ to $15 \mathrm{~d}-\mathrm{PGJ}_{2}$. 


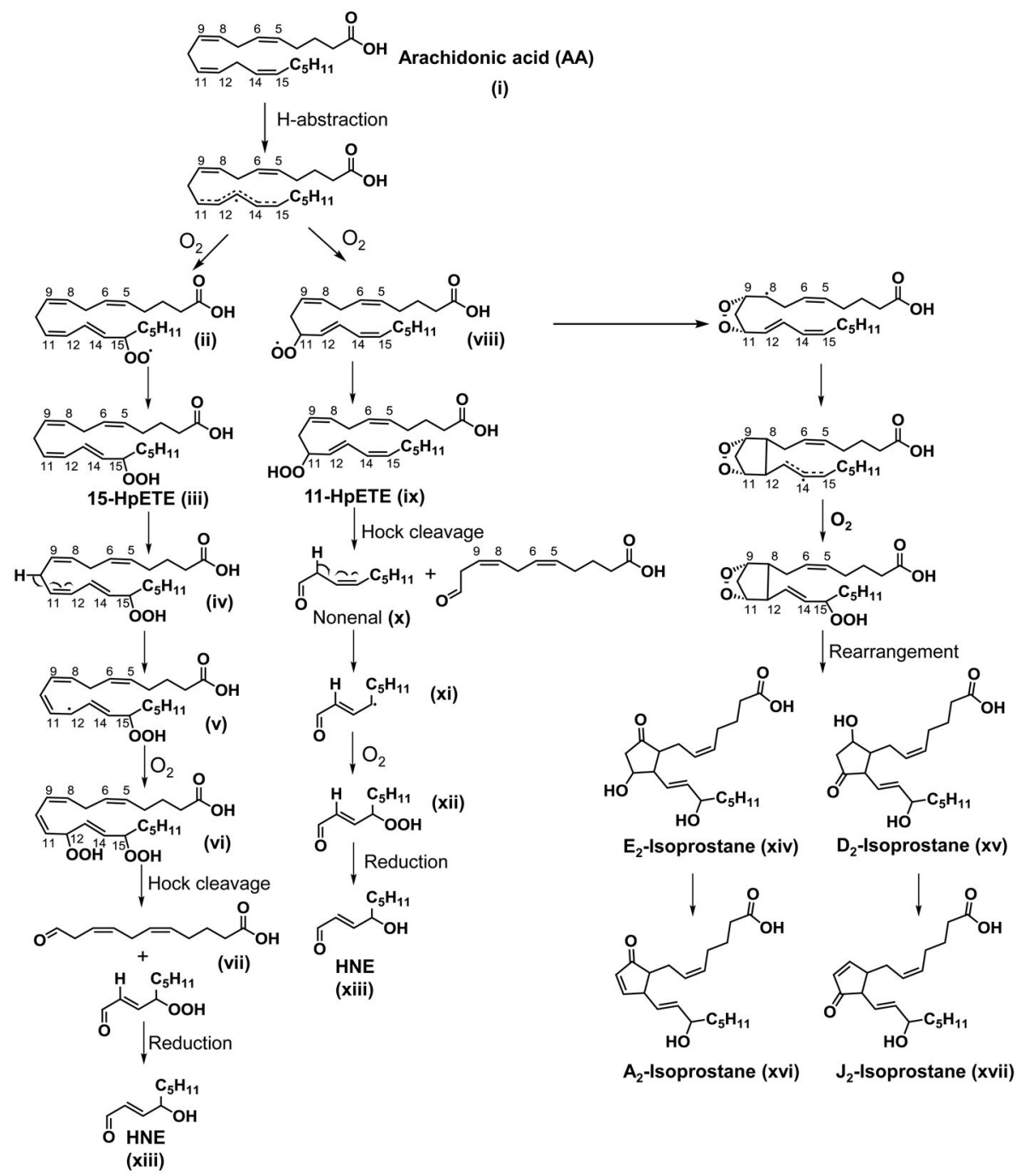

Figure 6.

Non-enzymatic generation of reactive lipid-derived electrophiles (LDEs): The generation of HNE (xiii) and $\mathrm{A}_{2}$-(xvi) and $\mathrm{J}_{2}$-isoprostanes (xvii) from $\omega$-6-FAs such as AA (i) is shown here. Reactive radicals such as ${ }^{\circ} \mathrm{OH}$ initiate lipid peroxidation by abstracting a bis-allylic hydrogen from $\mathrm{AA}$ (i). $\mathrm{O}_{2}$ addition at either $\mathrm{C}_{(15)}$ or $\mathrm{C}_{(11)}$ generates the corresponding peroxy radical (ii and viii), which abstracts an $\mathrm{H}$ radical (possibly from another $\mathrm{AA}$ molecule thus propagating the radical chain reaction) to generate the hydroperoxy intermediates 15-HpETE (iii) and 11-HpETE (ix), respectively. The abstraction of hydrogen at $\mathrm{C}_{(10)}$ of 15 -HpETE forms a radical intermediate which migrates to $\mathrm{C}_{(12)}$, reacts with $\mathrm{O}_{2}$, yielding the dihydroperoxide intermediate (iv). This intermediate then undergoes Hock cleavage to yield HNE as one possible product. 11-HpETE is shown to first undergo a Hock cleavage to generate the nonenal $(\mathbf{x})$, which undergoes oxygenation to yield the hydroperoxide (xii) and its further reduction to generate HNE (xiii). AA peroxidation also generates isoprostanes. The peroxy radical intermediate (viii) at $\mathrm{C}_{(11)}$ can undergo cyclization, further oxygenation, and rearrangement to yield $\mathrm{E}_{2}-(\mathbf{x i v})$ and $\mathrm{D}_{2}$-isoprostanes (xv). Dehydration of the $\mathrm{E}_{2}$ - and $\mathrm{D}_{2}$-isoprostanes result in $\mathrm{J}_{2}$ (xvi) and $\mathrm{A}_{2}$-isoprostanes 
(xvii), respectively. Analogous peroxidation pathway from $\omega$-3-FA such as DHA generates HHE. 


\section{Low oxygen}<smiles>C/C=C\C/C=C\C</smiles>

(i)<smiles>C/C=C\C=C/[C@@H](/C=C\C)C(/C=C\C(C)[N+](=O)[O-])=C\C=C/C</smiles>

Nitroalkane
$>$<smiles>CCC[N+](=O)[O-]</smiles><smiles>C=CCC(C)C(C)[N+](=O)[O-]</smiles>

(ii)

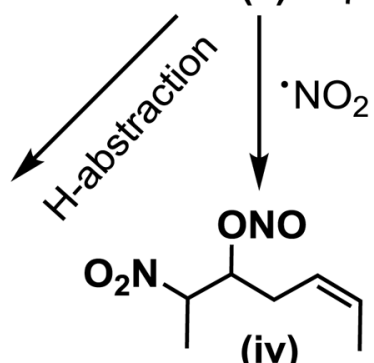

(iv)
High oxygen<smiles>C/C=C\C/C=C\C</smiles>

(i)<smiles></smiles><smiles>C/C=C\C=C/C=C\C</smiles><smiles>[O-][18O]</smiles><smiles>C/C=C\C=C\C(C)OO</smiles>

Lipid hydroperoxide (vii)<smiles>C/C=C\C/C=C(\C)[N+](=O)[O-]</smiles>

Nitroalkene

(v)

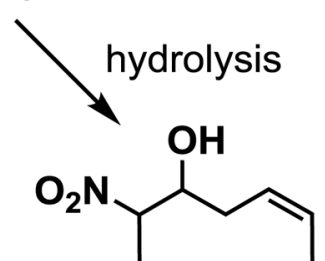

nitrohydroxy derivative (vi)

Figure 7.

Generation of $\mathrm{NO}_{2}$-FA from the alkyl portion of unsaturated FAs. Under low ${ }^{3} \mathrm{O}_{2},{ }^{\circ} \mathrm{NO}_{2}$ undergoes radical adduction with unsaturated FAs (i) (such as linoleic acid) to generate a nitroalkyl radical intermediate (ii). This intermediate can either abstract a hydrogen to generate nitroalkane (iii) or further react with ${ }^{\bullet} \mathrm{NO}_{2}$ to generate a nitro/nitrite intermediate (iv). Subsequent loss of nitrous acids (HONO) yields a nitroalkene (v) whereas hydrolysis of the intermediate generates a hydroxy-nitro FA (vi). Under high ${ }^{3} \mathrm{O}_{2}$, a lipid hydroperoxide (vii) is formed. 

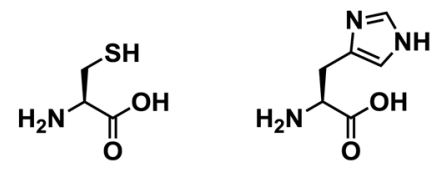

Cysteine

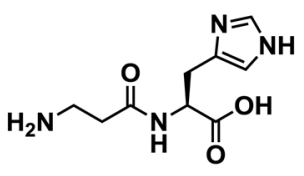

Carnosine

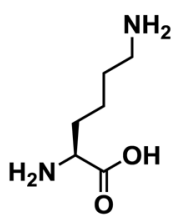

Lysine

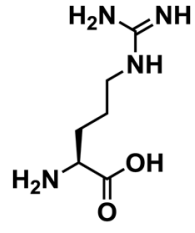

Arginine

Figure 8.

Nucleophilic amino acid and amino-acid derived molecules (in order of decreasing nucleophilicity) 


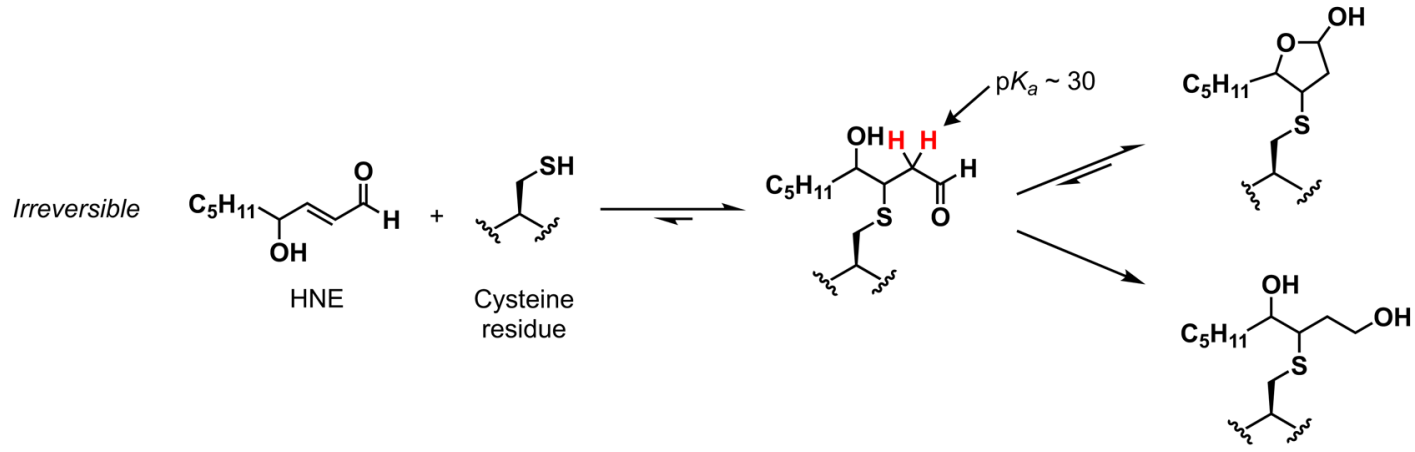

$\mathrm{C}_{5} \mathrm{H}_{11}$
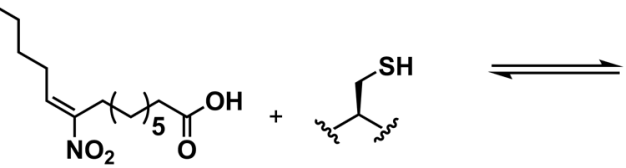

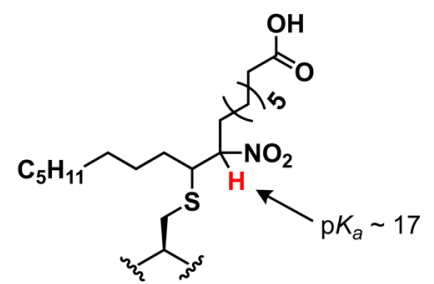

9-nitro-oleic Cysteine acid residue

Figure 9.

Reversible and irreversible RES conjugates: $\mathrm{p} K_{\mathrm{a}}$ of the $\mathrm{C}-\mathrm{H}$ proton(s) a-to the carbonyl or nitro group affects the reversibility of the thiol conjugates. In the case of the reduced HNEthiol adduct, the source of the reductant is currently unknown, but this modification has been reported by independent laboratories for the RES-sensor Keap1 following global treatment of cells with HNE. $(259,511)$ This modification was also detected following T-REXmediated HNE-delivery to another RES-sensor, Akt3.(512) See also Figure 13. 
A

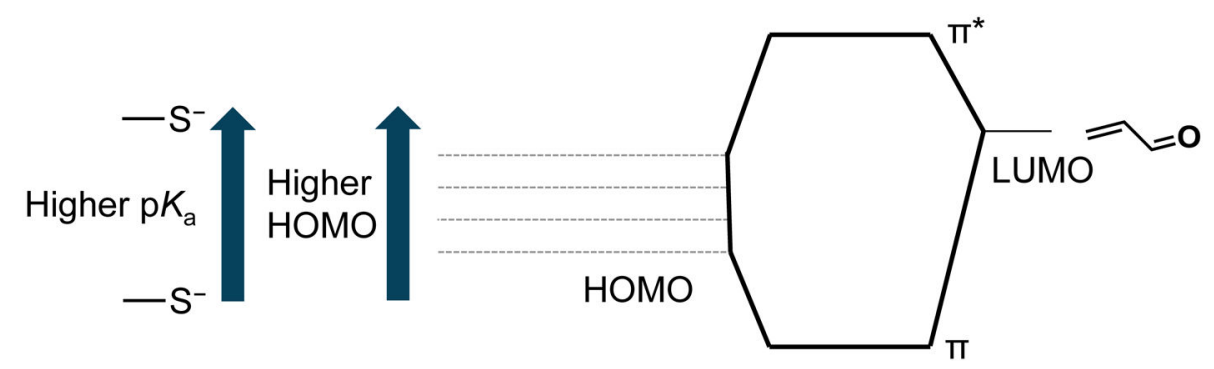

B

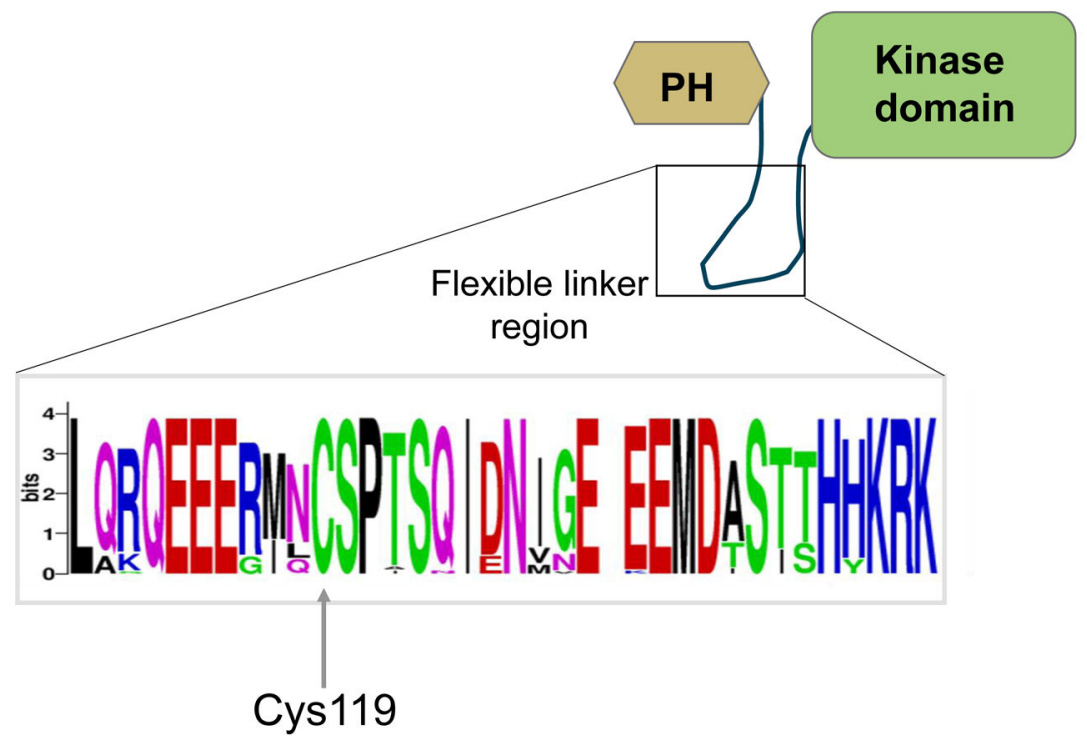

Figure 10.

Properties of a privileged redox sensor. (A) Although lowering of $\mathrm{p} K_{\mathrm{a}}$ is widely credited with increasing nucleophilicity of the sensing cysteine residues, other factors must be responsible for privileged redox sensing ability. Indeed, thiolate nucleophiles formed from thiols with higher $\mathrm{p} K_{\mathrm{a}}$ are more nucleophilic compared to those formed from low $\mathrm{p} K_{\mathrm{a}}$ thiols. Thiolate formed from higher $\mathrm{p} K_{\mathrm{a}}$ thiols have a higher HOMO resulting in a better overlap with the LUMO of the electrophile making them more nucleophilic. Other factors such as the extent of solvent exposure and the microenvironment of a sensor cysteine also affect privileged redox sensing (B). In Akt3, a privileged RES sensor, C119 located in the flexible linker region of the enzyme and surrounded by charged amino acids is a privileged HNE sensing residue. PH: Pleckstrin homology domain. Inset: Logo demonstrates the high sequence conservation of $\mathrm{C} 119$ and the surrounding charged residue reflecting their functional importance. Sequence alignment of 35 vertebrate Akt3 sequences was performed using Mega7.0. Logo was created using WebLogo (Berkeley). 


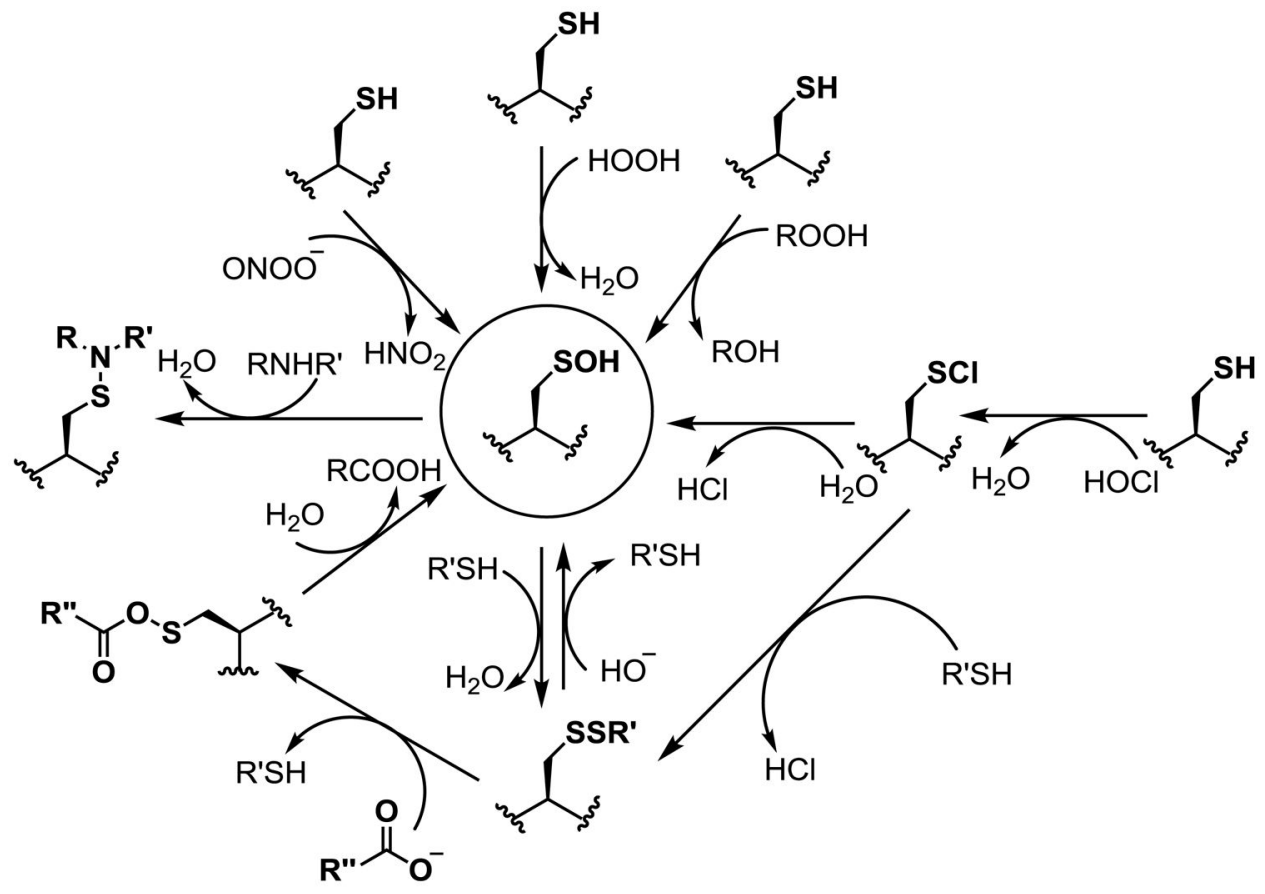

Figure 11.

Oxidation of cysteine by various oxidants generates sulfenic acid. Sulfenic acid can also act as an intermediate to other cysteine modifications including glutathionylation, disulfide bond formation, and sulfenamide species. 
A

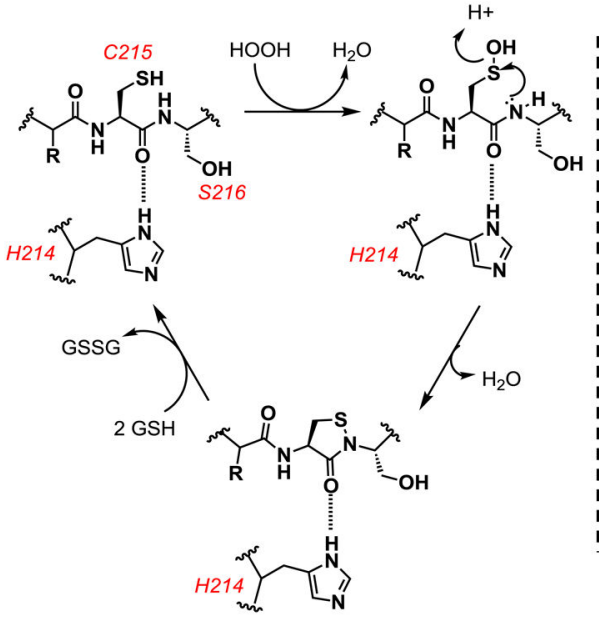

B

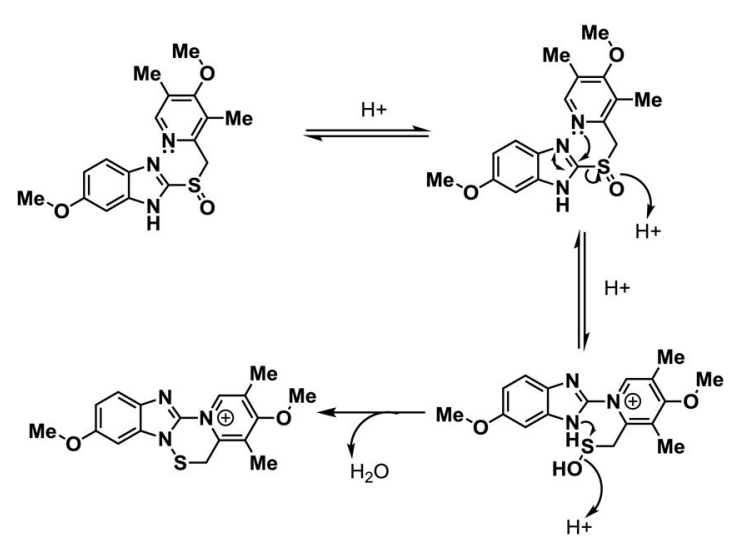

Figure 12.

(A) Schematic of sulfenylamide formation in enzyme catalytic pocket. Sulfenylamide formation at the catalytic sites of enzymes such as PTP1B prevents them from irreversible hyperoxidation. (B) A sulfenamide intermediate is also formed by the drug omeprazole. 

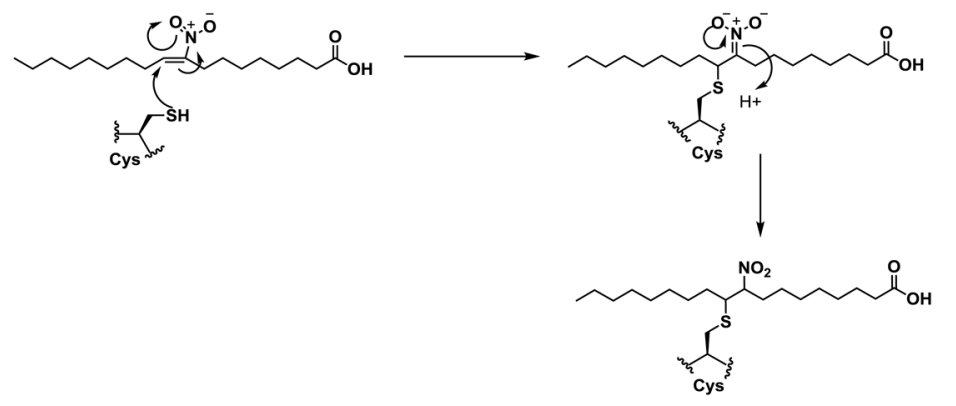

B

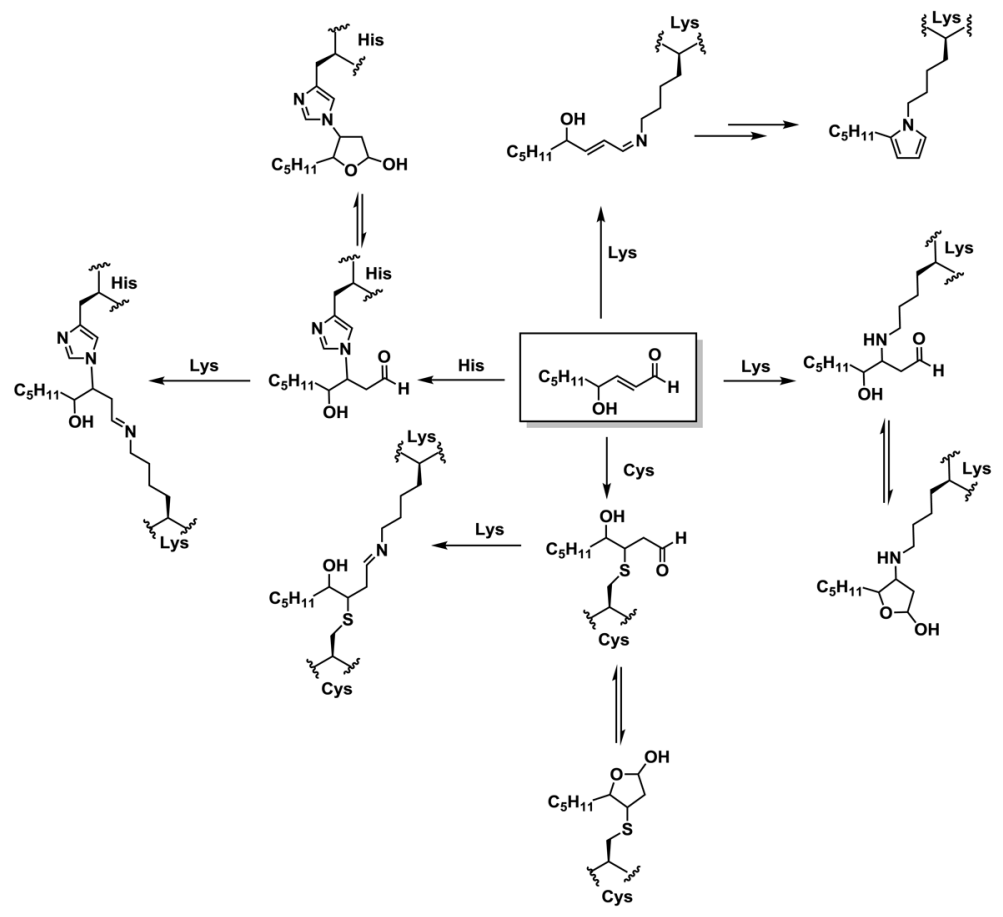

Figure 13.

Protein alkylation by (A) monofunctional RES, 9-nitroleic acid, versus (B) modifications by the multifunctional RES, HNE. See also Figure 9. 
A

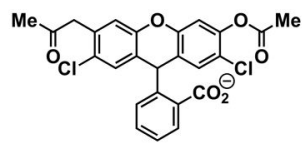
Dichlorodihydrofluorescein diester
(DCFH 2 -DA)
$\mathrm{H}_{2} \mathrm{~N}$

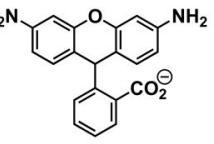

Dihydrorhodamine
(DHR)

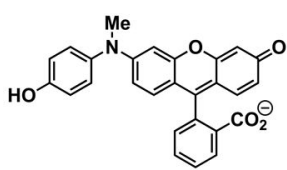

HK-Green4

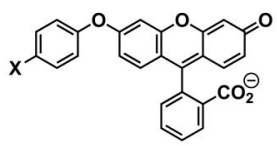

$\mathrm{X}=\mathrm{OH}, \mathrm{HPF}$
$\mathrm{X}=\mathrm{NH}_{2}, \mathrm{APF}$

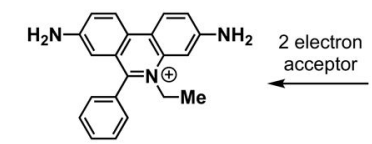

Ethidium ( $E+)$

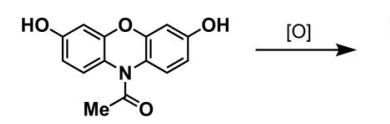

Amplex Red

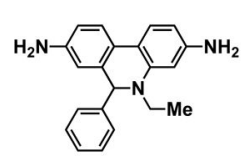

Hydroxyethidine (HE)

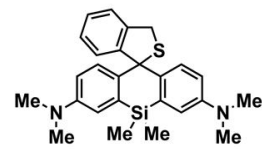

MMSiR

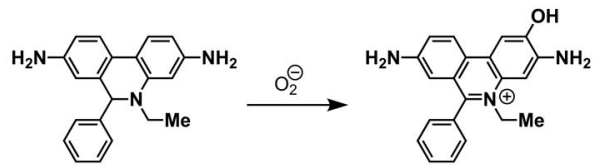

Hydroxyethidine (HE)

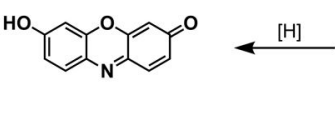

Resorufin
2-OH-ethidium (2-OH-E+)

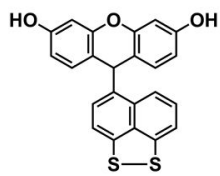

Redox Fluor1 (RF-1)

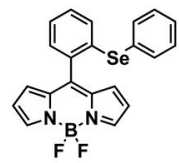

HCSe

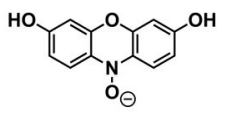

Resazurin (alamarBlue)

Figure 14.

(A) A selection of fluorescent probes for measuring ROS in cells. (B) Mechanism of Hydroxyethidine (HE) oxidation. Oxidation by superoxide generates $2-\mathrm{OH}-\mathrm{E}^{+}$with distinct spectral properties compared to Ethidium $\left(\mathrm{E}^{+}\right)$generated by a two-electron oxidation step by hydride acceptors. (C) Oxidation of Amplex Red ( $a n \mathrm{H}_{2} \mathrm{O}_{2}$ sensor) or reduction of resazurin (a cell viability reagent) leads to the fluorescent product resorufin. 
A
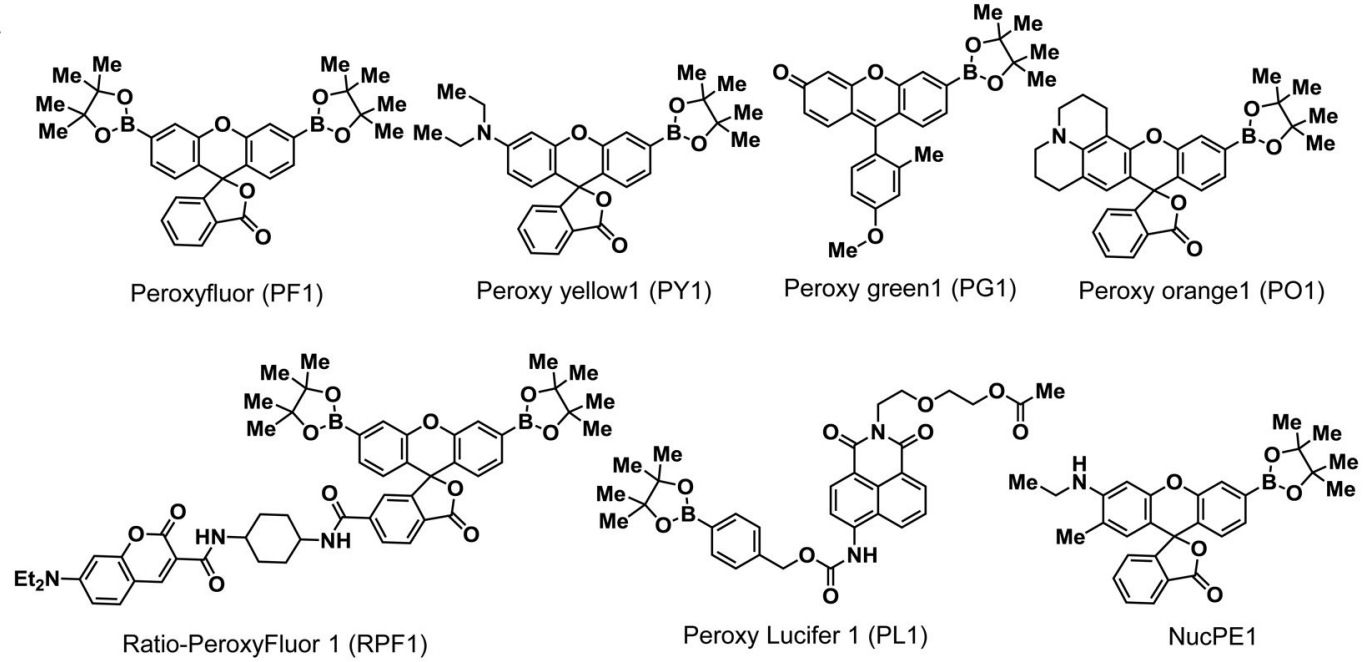

B

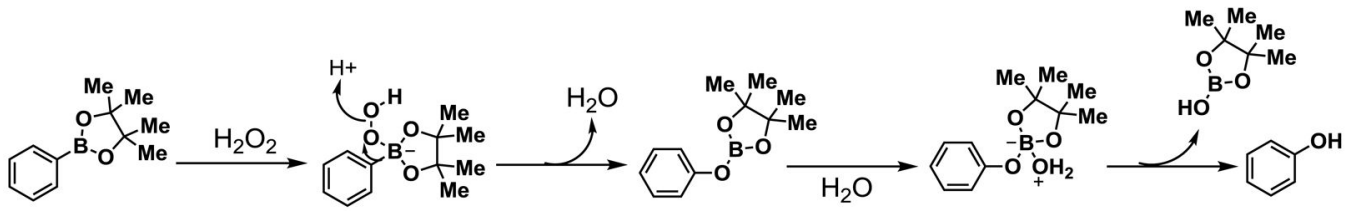

Figure 15.

(A) A selection of boron ":ate"-based fluorescent-probes for measuring $\mathrm{H}_{2} \mathrm{O}_{2}$ in cells. (B) Mechanism of boron "ate" probe oxidation by $\mathrm{H}_{2} \mathrm{O}_{2}$. 
A

Orp1-roGFP2

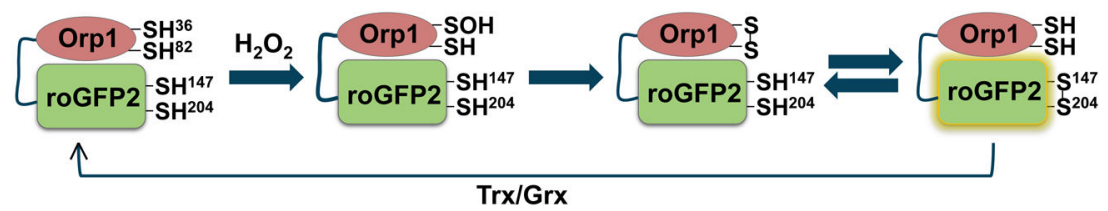

HyPer

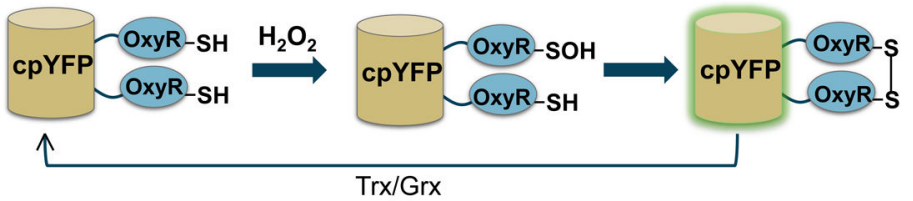

B
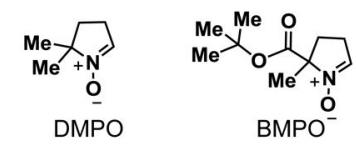

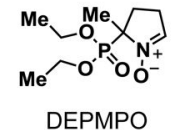

${ }_{\mathrm{NH}}^{\mathrm{Ph}_{3} \mathrm{P}^{+}}$
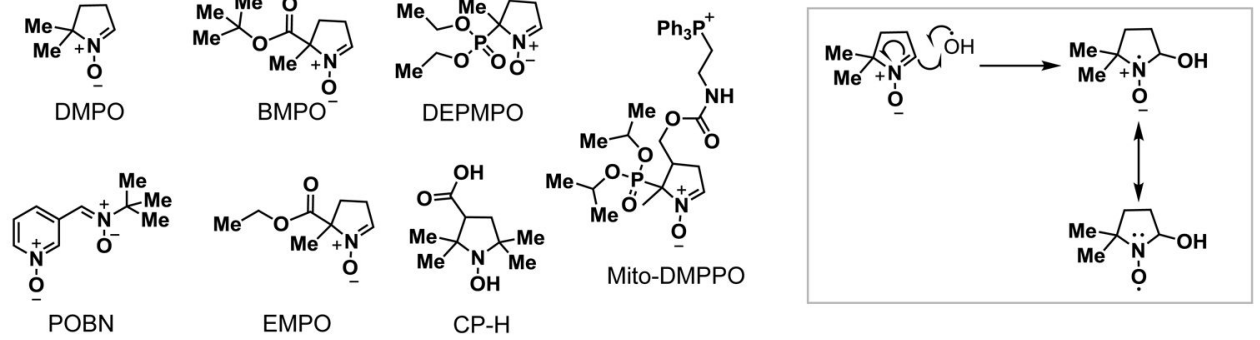

C
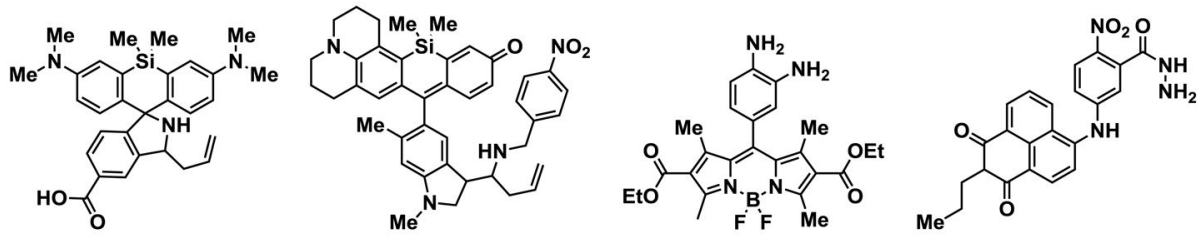

FAP-1

FP-1

Methyl diaminobenzene BODIPY (Mbo)

MDAP-1 


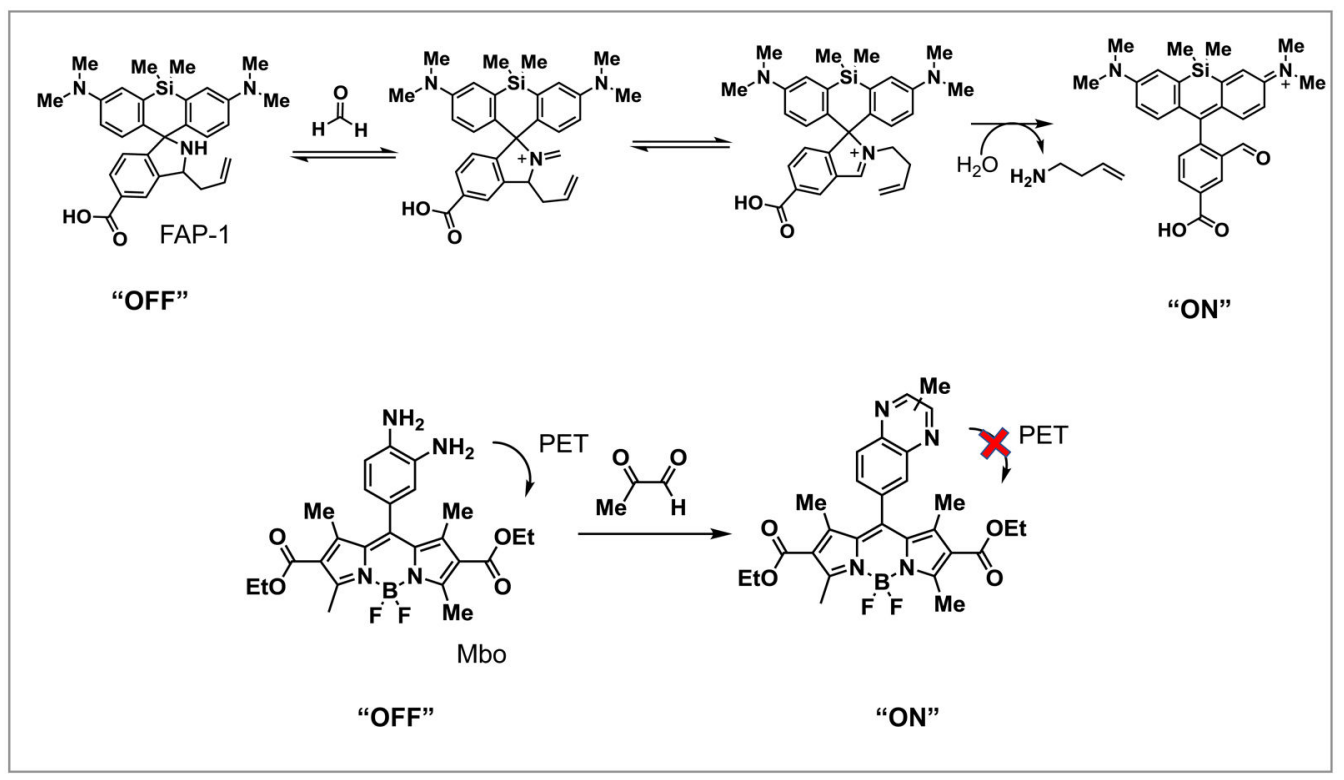

Figure 16.

(A) Examples of genetically-encoded $\mathrm{H}_{2} \mathrm{O}_{2}$ sensors. The reversibility of genetically-encoded sensors makes them an excellent tool to measure intracellular flux of $\mathrm{H}_{2} \mathrm{O}_{2}$. Top panel: Orp1-roGFP2 consists of a Orp1, a member of the yeast glutathione peroxidase, fused the redox-sensitive roGFP2. The peroxidatic cysteine on Orp (C36) forms a sulfenic acid in presence of $\mathrm{H}_{2} \mathrm{O}_{2}$, which is rapidly condensed by the resolving C82 of Orp1. This redox relay is transferred to the conjugated roGFP2 through a thiol-disulfide exchange. Disulfide bond formation of roGFP2 results in conformational change and a gain in fluorescence. Oxidized Orp1 is reversible by Trx. Oxidized roGFP2 can be reversed by Grx. Bottom panel: HyPer uses a circularly permutated YFP protein fused in between the regulatory domain of $\mathrm{OxyR}$, a bacterial $\mathrm{H}_{2} \mathrm{O}_{2}$-sensing transcription factor. The peroxidatic cysteine of OxyR (C199) is oxidized by $\mathrm{H}_{2} \mathrm{O}_{2}$ and resolved by $\mathrm{C} 208$ forming a disulfide bond. The consequent change in conformation of the conjugated cpYFP results in increase in fluorescence. Trx: Thioredoxin; Grx: Glutaredoxin. (B) A selection of EPR probes used for detecting radical RES and ROS generation in cells. Inset: Mechanism of radical trapping by cyclic nitrone spin traps to generate a stable nitroxide radical (C) Examples of fluorescent probes for the detection of free RES in cells. Inset: Top: Mechanism of 2-aza-cope reaction based fluorescent probes for detecting formaldehyde. Bottom: Mechanism of Mbo, a 'turnon' fluorescent probe for methylglyoxal. Other hydrazine-based probes for detecting malondialdehyde and formaldehyde work on a similar principle. 

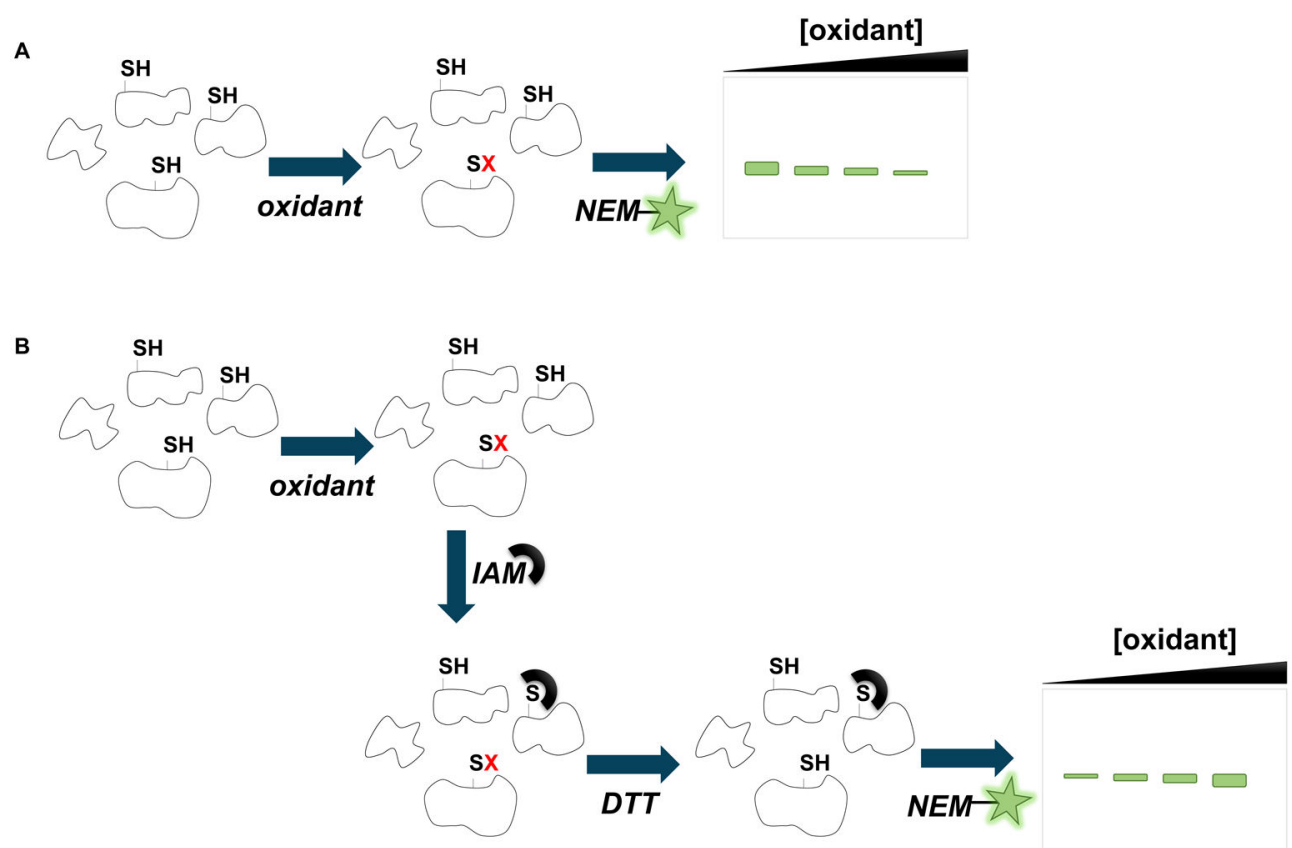

c Control

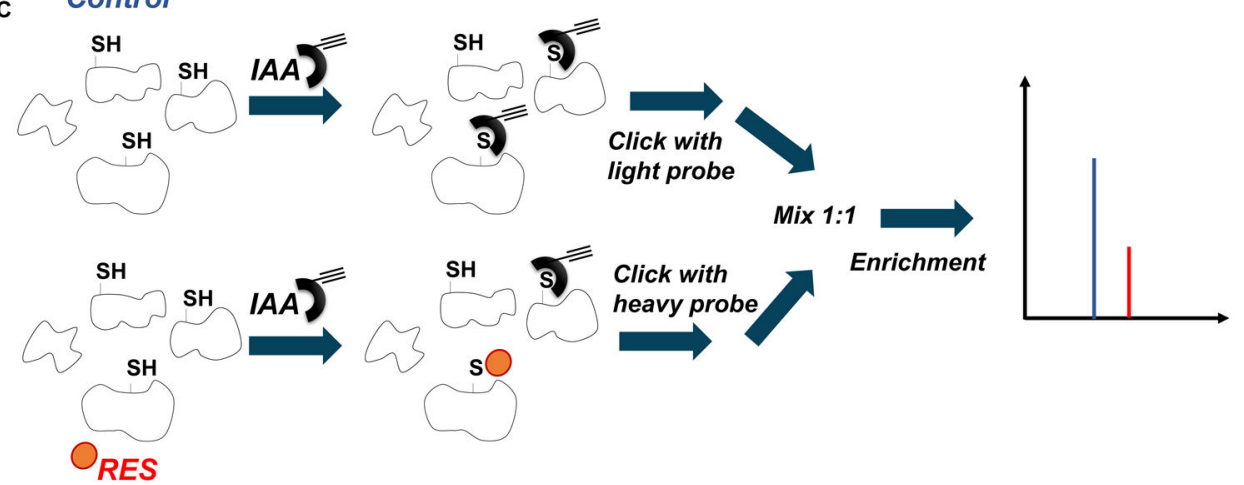

Figure 17.

Indirect methods to detect oxidative and electrophilic modifications of proteins (A) Oxidative and electrophilic modification of proteins renders them unreactive to a biotin/ fluorophore conjugated alkylating agent such as $\mathrm{N}$-ethyl maleimide (NEM)-Fluorescein. Modified proteins are detected from the loss in signal intensity using SDS-PAGE analysis (B) Similar to (A) except unmodified proteins are first capped using a broadly-reactive alkylating agent such as iodoacetamide (IAM, denoted by black cap). The oxidative modification $(\mathrm{X})$ on proteins is then reduced using a strong reducing agent such as DTT. A fluorophore/biotin-conjugated probe is used to label the reduced proteins. An increase in signal intensity is detected using SDS-PAGE/western blot corresponding to the increase in modification of protein with increasing oxidant concentration. (C) Isotope-labeling methods coupled with mass spectrometric approaches allow quantitative determination of protein modification. In methods such as competitive-isoTOP ABPP, the experimental sample is treated with the desired RES (red/orange circle) and the control sample is treated with the vehicle. The unreacted cysteines in the protein lysate are then capped with an alkylating agent with an alkyne handle (IA alkyne; IAA which caps most but not necessarily all 
remaining cysteines) which enables click-coupling with isotope-labeled and TEV-proteasecleavable-biotin azide. The samples are then mixed 1:1, enriched using streptavidin beads, the bound proteins are eluted using TEV protease, and a MS analysis performed subsequent to trypsin digestion. Proteins labeled with RES won't react with alkyne-tagged alkylating agents resulting in a loss in signal intensity in MS spectrum. 
A<smiles>CC(C)=O</smiles>

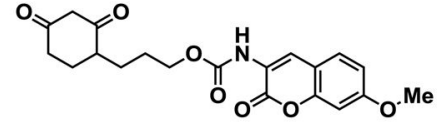

DCP-MCC<smiles>O=C1CCC(CCCOC(=O)CCCCC2SCC3NC(=O)NC32)C(=O)C1</smiles>

DCP-Bio1
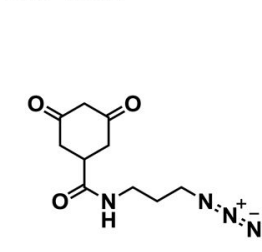

DAz-1
B

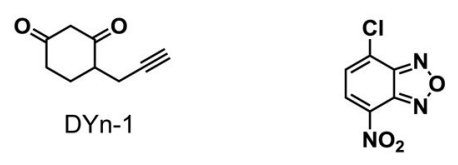

NBD-Cl

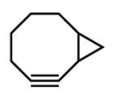

$\mathrm{BCN}$

c

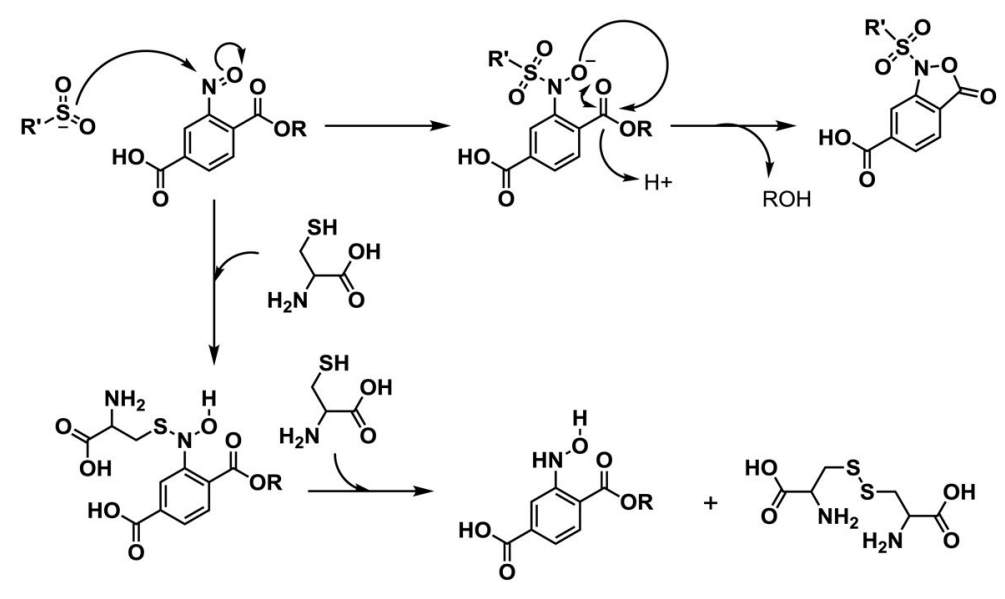

Figure 18.

(A) A selection of dimedone-based nucleophilic probes and (B) electrophilic probes for detecting sulfenic acid formation. (C) Aryl nitroso compounds enable detection of sulfinic acid although their reactivity with reduced thiol may limit their ability to detect sulfinic acid in biological systems. Capping reduced thiols with alkylating agents prior to probe treatment may mitigate this problem. A biotinylated aryl nitroso probe has recently been used for detection of sulfinic acid modified proteins in biological samples. 
A

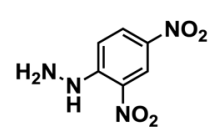

DNPH

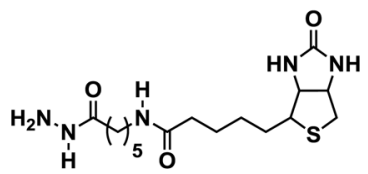

Biotin hydrazide<smiles>NC(=O)c1ccccc1C1c2ccc(O)cc2Oc2cc(O)ccc21</smiles>

Fluorescein hydrazide

B

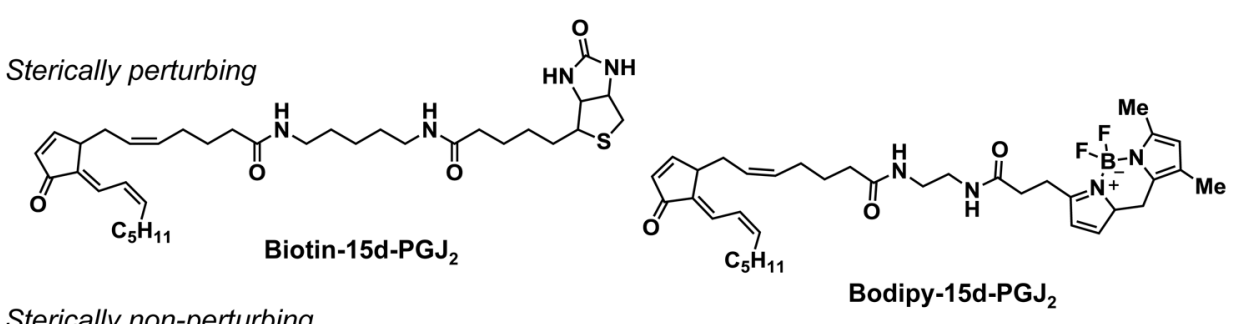

Sterically non-perturbing
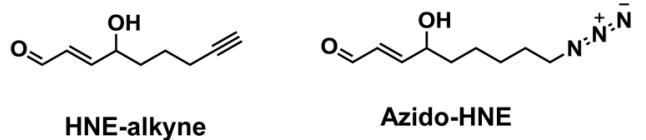

Azido-HNE

C

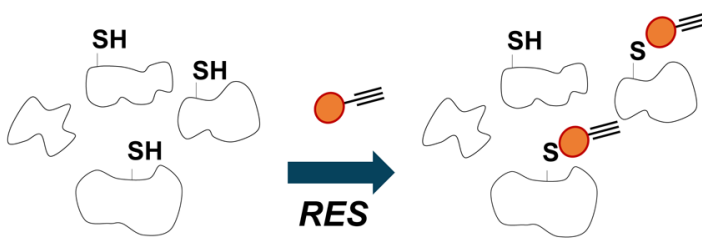

Click
coupling
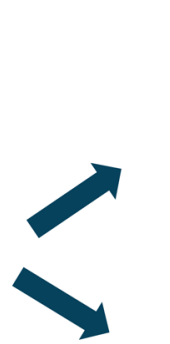

Enrichment

\section{Figure 19.}

(A) A selection of nucleophilic probes used for aldehyde capture of proteins-modified with endogenous electrophilic signals. Biotinylated and fluorescently-labeled hydrazide can be used for aldehyde capture for detection by WB/mass spectrometry and in-gel fluorescence, respectively. (B) Biotinylated and fluorescently-labeled LDEs can also be used to directly enrich or detect protein targets. Alternatively, alkyne and azide functional groups provide largely non-intrusive handles to assess protein targets of electrophiles. (C) Schematic of direct methods for profiling LDE sensitive cysteines in the proteome. Cells/lysates are treated with an excess of alkyne-functionalized RES (red diamond). Modified proteins are either observed using in-gel fluorescence after click-coupling with fluorophore-azide or enriched using streptavidin beads post click coupling with biotin-azide. Subsequent tandemMS reveals the modified peptides, and depending on the experimental context, the site(s) of modification. 
A

In live cultured cells, fish, and worms
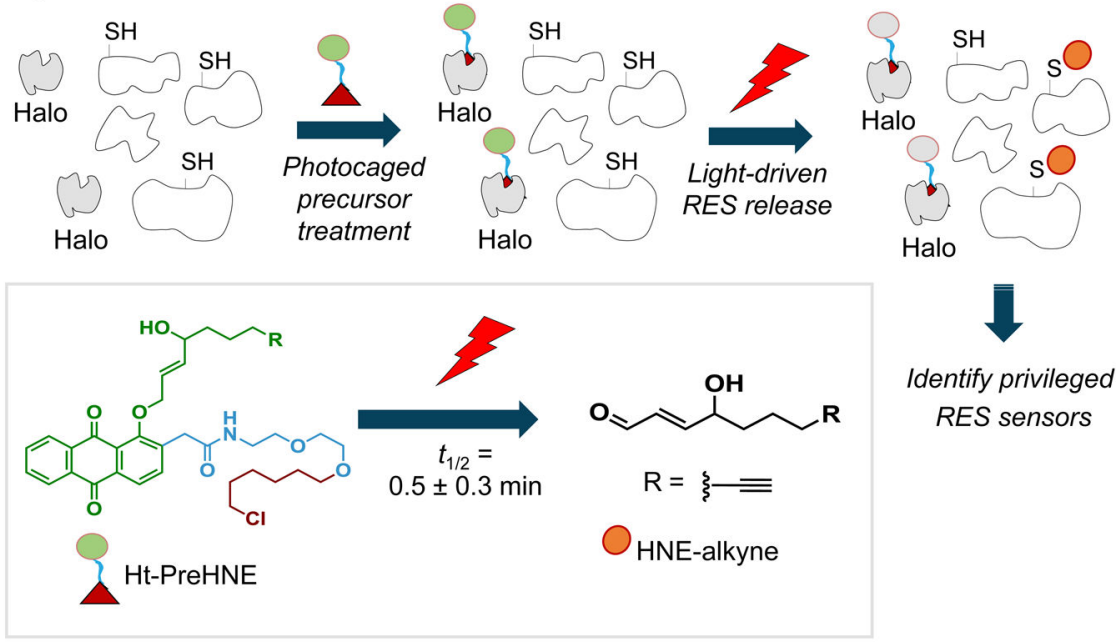

Identify privileged RES sensors

B

In live cultured cells, fish, and worms
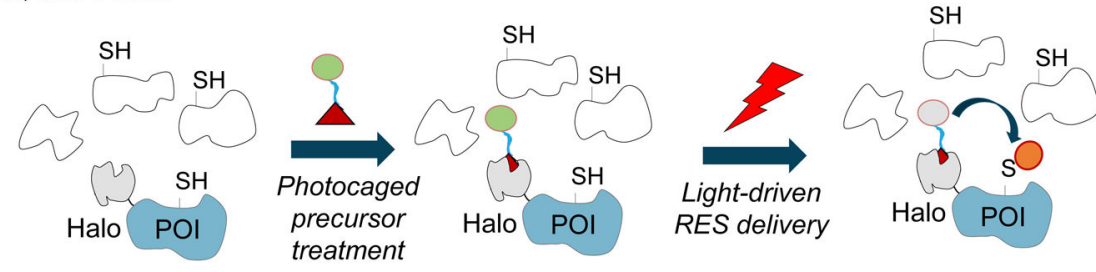

Evaluate target- and signal-specific response

Figure 20.

Workflow of G/T-REX. (A) G-REX employs expression of HaloTag in live cells to bind a non-invasive photocaged precursor to HNE (Ht-PreHNE; see inset) and release maximally 5 $\mu \mathrm{M}$ HNE in situ upon photoactivation. This method can be coupled to proteomics to globally profile privileged RES sensors. (B) T-REX is built on a proximity-targeting concept of a freely-diffusible RES (demonstrated thus far for various native bioactive LDEs such as HNE) to a specific sensor protein-of-interest (POI). As such, T-REX enables low-occupancy on-target RES-modifications at a user-defined time and space in living systems which can be directly linked to functional redox responses (thus far proven compatible with live cells, fish, and worms). Under these native electrophile-limited settings, competition is set up between innate diffusivity of RES and reactivity (kinetic privilege) of the sensor protein/cysteine. TREX involves incubating the live cells/animals expressing functional HaloTagged-POI with sub-micromolar bioinert bifunctional photocaged precursors to an LDE such as HNE (see Inset) non-invasively, followed by rinsing out the excess probe unbound to Halo, and subsequent light exposure. The latter rapidly liberates the LDE in the amount at maximum stoichiometric to in vivo concentration of Halo-POI within the coordination shell of the 
Halo-POI. Built-in controls ensure/account for no off-target labeling/responses.

$(252,259,389,512,609,957,958)$ Signaling consequences resulting from the on-target LDE modification of a privileged LDE-sensor POI have been shown to be evaluated using various downstream readouts.

Inset: One terminus of the bifunctional photocaged probe (exemplified for HNE) consists of a hexyl chloride linker (brown) that can covalently conjugate with high specificity and affinity to a HaloTag (gray) genetically fused to a POI (blue). The other terminus consists of photocaged-LDE which upon photouncaging using a low-power UV lamp generates LDE such as HNE. 


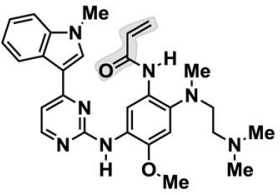

Osimertinib (2015) NSCLC

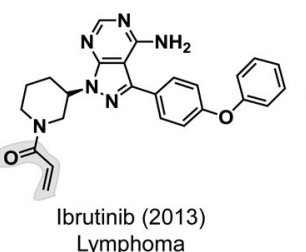

Lymphoma

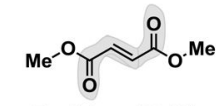

Tecfidera (2013)

Relapsing remitting MS

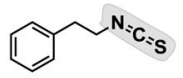

Phenethyl isothiocyanate (Phase II)

Lung cancer

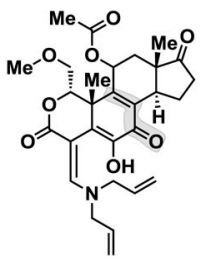

PX-866 (Phase I/II) Various cancers

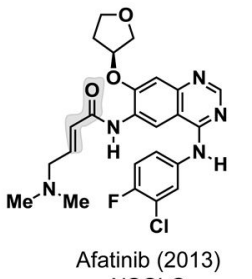
NSCLC

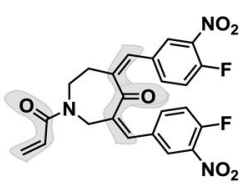

VLX1570 (Phase I/II)
Relapsed multiple myeloma

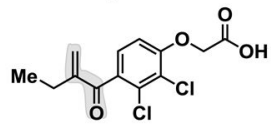

Ethacrynic acid (Prior to 1982) Diuretic

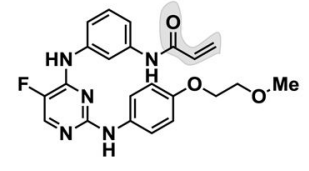

CC-292 (Phase I/II) Rheumatoid arthritis, B-cell Lymphoma

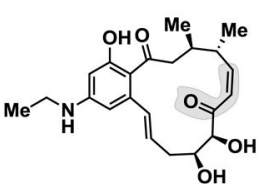

E6201 (Phase I/II) Leukemia

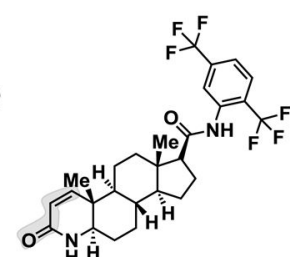

Dutasteride (2001) Benign prostate hyperplasia

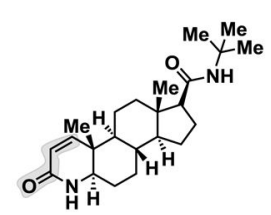

Finasteride (1992) Benign prostate hyperplasia

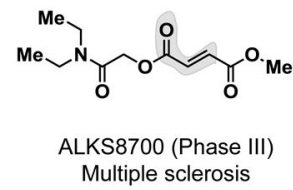

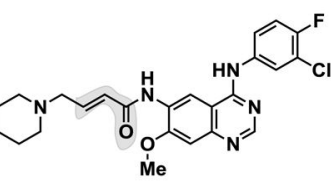

Dacomitinib (Phase III) NSCLC

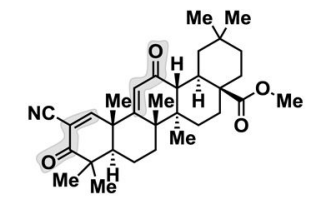

Bardoxolone methyl (Phase I/II)

Pulmonary hypertension

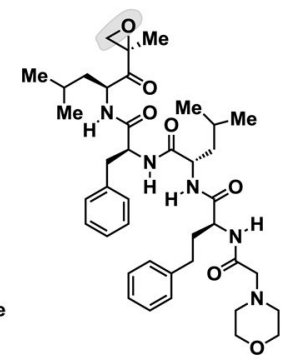

Carfilozomib (2012) Advanced multiple myeloma

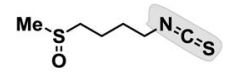

Sulforaphane (Phase II) Prostate cancer, Autism

Figure 21.

Small molecules bearing electrophilic pharmacophores approved by the FDA (year approved in parenthesis) or currently in clinical trials in the treatment of indicated disease. HER2 ${ }^{+}$: human EGFR-2-positive. MS: Multiple sclerosis. NSCLC: Non-small cell lung carcinoma. 


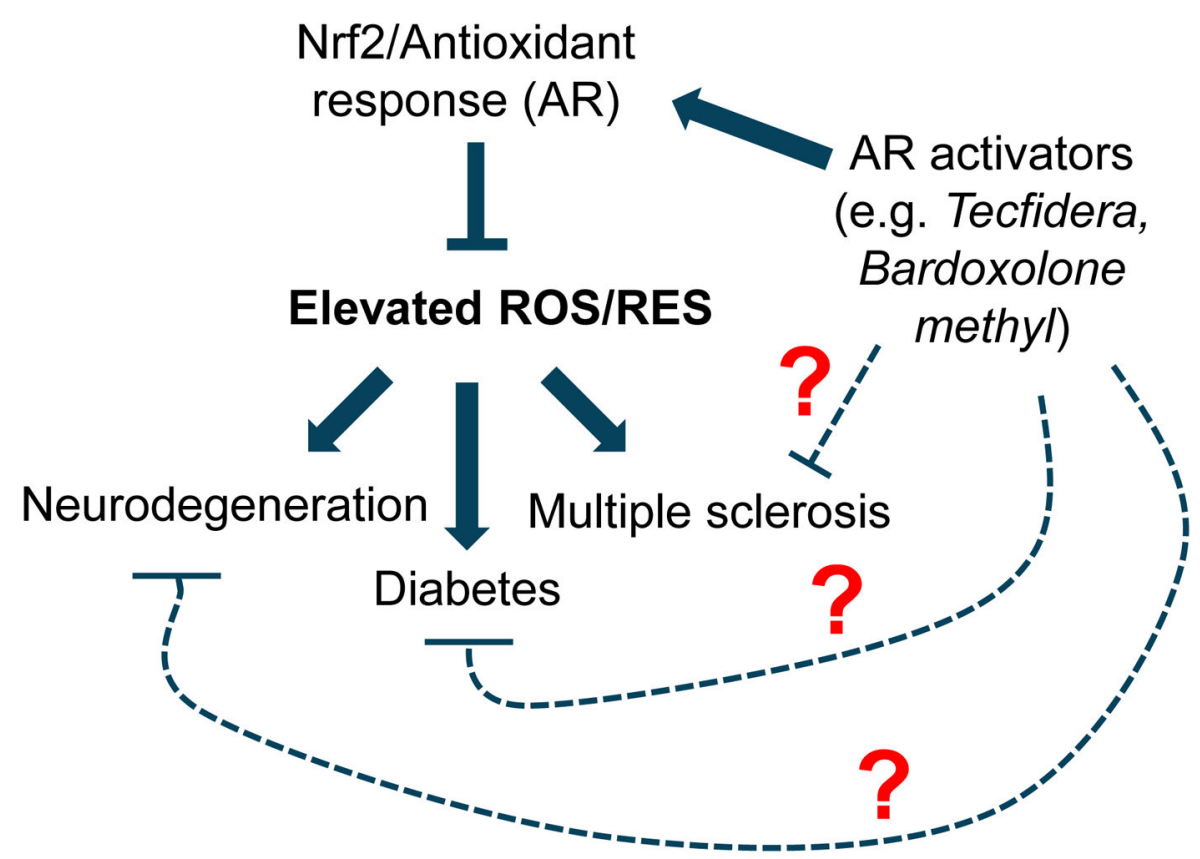

Figure 22.

Elevated ROS and RES levels are markers of various diseases including diabetes, multiple sclerosis, and neurodegenerative disorders. Activation of ROS/RES detoxification pathways such as the Nrf2/AR has shown promise in ameliorating these diseases. However, pathways other than Nrf2/AR may play important role in mediating the beneficial effects of broadspecificity electrophilic drugs/drug candidates such as Tecfidera. 


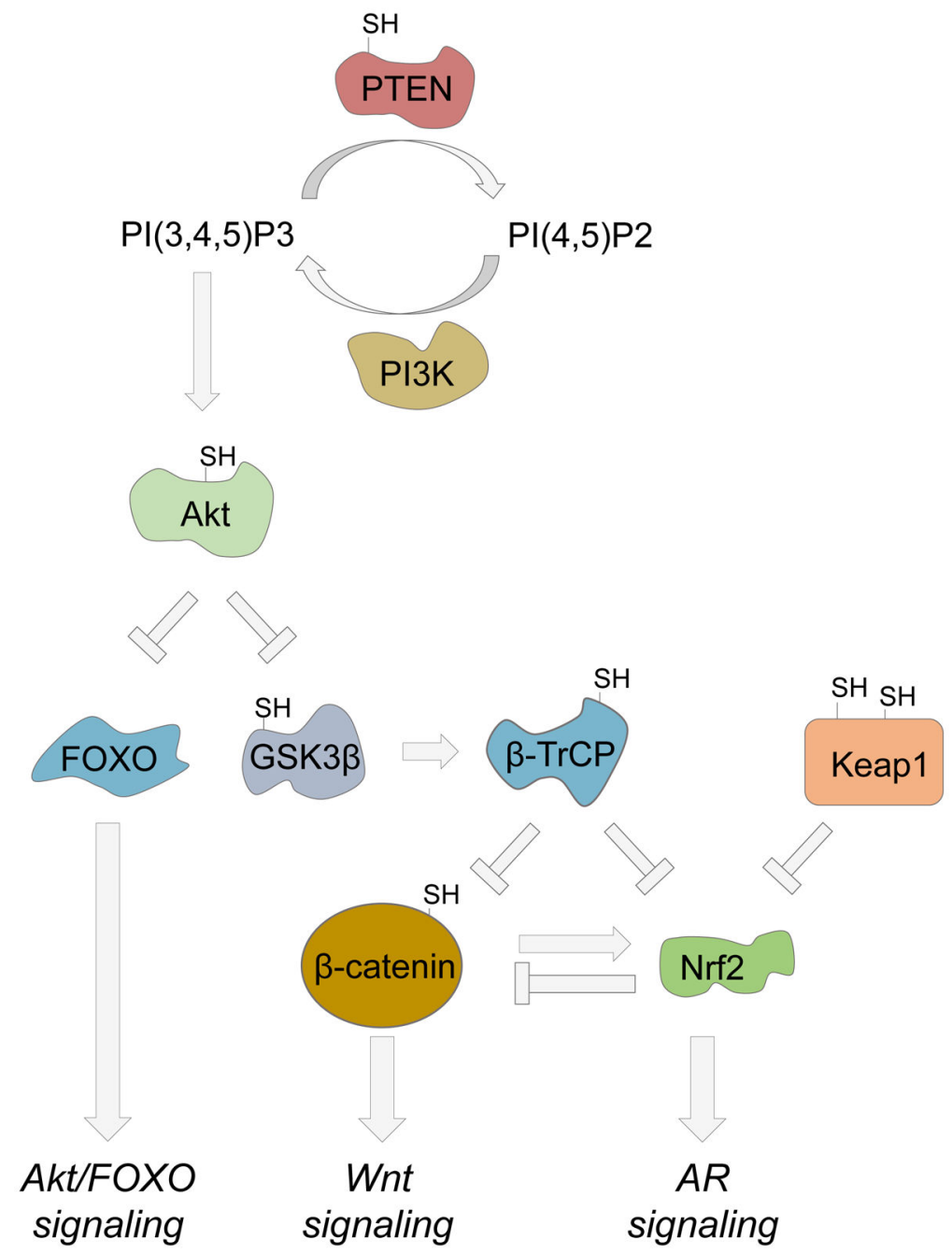

Figure 23.

Nrf2/AR signaling is at the crossroads of Wnt and Akt/FOXO signaling pathways. Nrf2, the master transcriptional regulator of the AR, is controlled by multiple redox-sensitive proteins. Keap1 negatively regulates AR signaling by targeting Nrf2 for degradation under non-stress conditions. Nrf2 is also regulated by the Akt signaling axis: activation of the Akt pathway triggers GSK3 $\beta$-mediated phosphorylation of Nrf2, resulting in the degradation of Nrf2 mediated by $\beta$-TrCP. Recent studies have identified the role of $\beta$-catenin, another substrate of $\beta$-TrCP, in regulating Nrf2/AR. Nrf2 inhibits $\beta$-catenin-mediated Wnt signaling, and $\beta$ catenin stimulates Nrf2-dependent AR. 


\section{Table 1}

Comparison of various endogenously-generated ROS and RES: their estimated cellular concentrations, halflives, diffusion distances in plasma or aqueous buffers, and second-order reaction rate constants (with $\mathrm{pH}$ and temperature at which the rates were determined given when possible) with biological molecules such as GSH. [For reported second-order reaction rate constants between a representative RES, 4-hydroxynonenal (HNE), and proteins, see Table S1].

\begin{tabular}{|c|c|c|c|c|}
\hline Species & Cellular concentration & Half-lives* & Diffusion distance ${ }^{* * *}$ & $\begin{array}{l}\text { Second-order rate } \\
\text { constants with } \\
\text { biological molecules } \\
\mathbf{M}^{-1} \mathrm{~s}^{-1}\end{array}$ \\
\hline $\mathrm{O}_{2}^{\cdot-}$ & $10^{-9}-10^{-12} \mathrm{M}(58)$ & $10^{-6} \mathrm{~s}(24,59,60)$ & Maximally $0.5 \mu \mathrm{m}(59)$ & $\begin{array}{l}\text { Variable (see text) } \\
\sim 200 \mathrm{M}^{-1} \mathrm{~s}^{-1} \text { with GSH } \\
\text { at } \mathrm{pH} 7.0,37^{\circ} \mathrm{C} \\
(61,62) \\
<10^{3} \mathrm{M}^{-1} \mathrm{~s}^{-1} \text { with NAC } \\
\text { at } \mathrm{pH} 7.4,25^{\circ} \mathrm{C}(63)\end{array}$ \\
\hline $\mathrm{H}_{2} \mathrm{O}_{2}$ & $\begin{array}{l}10^{-8}-10^{-9} \mathrm{M}(24) \\
10^{-7} \mathrm{M} \text { (stimulated)(24) }\end{array}$ & $\begin{array}{l}\left.10^{-3}-10^{-5} \text { s ( } 24\right) ; \\
\text { possibly longer in some } \\
\text { cells/under some } \\
\text { conditions (64) }\end{array}$ & $\begin{array}{l}1 \mu \mathrm{m}(65) ; \text { possibly } \\
\text { longer in some cells }\end{array}$ & $\begin{array}{l}\sim 1 \mathrm{M}^{-1} \mathrm{~s}^{-1} \text { with GSH at } \\
\mathrm{pH} 7.4,37^{\circ} \mathrm{C}(66)\end{array}$ \\
\hline$\cdot \mathrm{OH}$ & $10^{-9}-10^{-15} \mathrm{M}(58)$ & $10^{-9} \mathrm{~s}(67)$ & $0.001-0.005 \mu \mathrm{m}(67)$ & $\begin{array}{l}10^{9}-10^{10} \mathrm{M}^{-1} \mathrm{~s}^{-1} \text { with } \\
\mathrm{GSH} \text { at } \mathrm{pH} 7.0,22^{\circ} \mathrm{C} \\
\text { and NAC at } \mathrm{pH} 7.4, \\
25^{\circ} \mathrm{C}(68,69)\end{array}$ \\
\hline $\mathrm{ONOO}^{-}$ & $10^{-9} \mathrm{M}(70)$ & $10^{-3} \mathrm{~s}(71-73)$ & $5-20 \mu \mathrm{m}(72,73)$ & $\begin{array}{l}>10^{3} \mathrm{M}^{-1} \mathrm{~s}^{-1} \text { with } \\
\text { cysteine at } \mathrm{pH} 7.4 \\
37^{\circ} \mathrm{C}(72)\end{array}$ \\
\hline${ }^{1} \mathrm{O}_{2}$ & Not determined & $\begin{array}{l}4 \times 10^{-6} \mathrm{~s} \text { in water }(74- \\
76) \\
1.4 \times 10^{-5} \mathrm{~s} \text { in lipids } \\
(77) \\
3 \times 10^{-6} \mathrm{~s} \text { in nuclei/ } \\
\text { cells }(77,78) \\
\text { Possibly as short as } \\
0.2 \times 10^{-6} \mathrm{~s} \text { in cells }(79)\end{array}$ & $\begin{array}{l}\text { Between } 0.01 \text { and } 0.25 \\
\mu \mathrm{m}(76,80) \text {; up to } 0.4 \mu \mathrm{m} \\
\text { in lipids. (81) }\end{array}$ & $\begin{array}{l}10^{7} \mathrm{M}^{-1} \mathrm{~s}^{-1} \text { with } \\
\text { cysteine/Methionine at } \\
\mathrm{pH} 7.4,37^{\circ} \mathrm{C}(82)\end{array}$ \\
\hline $\mathrm{HOCl}$ & & $\begin{array}{l}\text { ca. } 60 \mathrm{~s} \text { in cells (see } \\
\text { text) }(47)\end{array}$ & $\begin{array}{l}\sim 0.03 \mu \mathrm{m} \text { in presence of } \\
\text { millimolar concentration } \\
\text { of thiols }(83)\end{array}$ & $\begin{array}{l}\sim 10^{8} \mathrm{M}^{-1} \mathrm{~s}^{-1} \text { with } \\
\text { cysteine/GSH/ } \\
\text { Methionine at pH } 7.4, \\
22^{\circ} \mathrm{C}(47,49)\end{array}$ \\
\hline HNE & $\begin{array}{l}8 \times 10^{-6} \mathrm{M}(84) \\
(0.3-1) \times 10^{-6} \mathrm{M}(85-87) \\
1.5 \times 10^{-5} \mathrm{M} \\
5 \times 10^{-3} \mathrm{M} \text { (stressed) }(85)\end{array}$ & $\begin{array}{l}<4 \mathrm{~s} \text { in rat heart }(85) \\
30 \mathrm{~s} \text { in hepatocyte } \\
\text { suspension }(88)\end{array}$ & $\begin{array}{l}0.010-0.100 \mu \mathrm{m} \text { in lipid } \\
\text { prior to movement to } \\
\text { cytosol; }(89) \text { likely } \\
\text { several } \mu \mathrm{m} \text { (longer than } \\
\mathrm{H}_{2} \mathrm{O}_{2} \text { ) }\end{array}$ & $\begin{array}{l}0.0021 \mathrm{M}^{-1} \mathrm{~s}^{-1} \text { with } \mathrm{N}- \\
\text { acetyl histidine; } \\
0.0013 \mathrm{M}^{-1} \mathrm{~s}^{-1} \text { with } \mathrm{N}- \\
\text { acetyl lysine; } 1.33 \mathrm{M} \\
{ }^{-1} \mathrm{~s}^{-1} \text { with } \mathrm{GSH} ; 1.21 \\
\mathrm{M}^{-1} \mathrm{~s}^{-1} \text { with cysteine, } \\
\text { all at } \mathrm{pH} 7.4,23^{\circ} \mathrm{C} \\
\text { (90) }\end{array}$ \\
\hline Nitro-Fatty acids & $\begin{array}{l}\sim 10^{-9} \mathrm{M} \text { (nitro-conjugated } \\
\text { linoleic acid) in human plasma } \\
\text { and }<10^{-8} \mathrm{M} \text { in human urine } \\
(91-93) \\
\sim 1.7 \times 10^{-8} \mathrm{M} \text { of nitro- } \\
\text { conjugated linoleic acid in } \\
\text { heart tissue under stress }(94) \\
\sim 9.5 \times 10^{-9} \mathrm{M} \text { of free nitro- } \\
\text { oleic acid in heart tissue under } \\
\text { stress }(91) \\
\sim 6 \times 10^{-7} \mathrm{M} \text { of free nitro-oleic } \\
\text { acid in plasma }(91)\end{array}$ & $\begin{array}{l}\text { Nitro-linoleic acid } \\
\sim 1.8 \times 10^{3} \mathrm{~s} \text { in } \\
\text { phosphate buffer at } \mathrm{pH} \\
7.4,37^{\circ} \mathrm{C}(91) \\
\text { Nitro-oleic acid } \\
\sim 7.2 \times 10^{3} \mathrm{~s} \text { in } \\
\text { phosphate buffer at } \mathrm{pH} \\
7.4,37^{\circ} \mathrm{C}(91)\end{array}$ & Not determined & $\begin{array}{l}\sim 350 \mathrm{M}^{-1} \mathrm{~s}^{-1} \text { for GSH } \\
\text { with nitro-linoleic acid } \\
\text { at } \mathrm{pH} 7.4,37^{\circ} \mathrm{C}(95) \\
\sim 180 \mathrm{M}^{-1} \mathrm{~s}^{-1} \text { for } \mathrm{GSH} \\
\text { with nitro-oleic acid at } \\
\text { pH } 7.4,37^{\circ} \mathrm{C}(95)\end{array}$ \\
\hline
\end{tabular}




\begin{tabular}{|c|c|c|c|c|}
\hline Species & Cellular concentration & Half-lives ${ }^{*}$ & Diffusion distance ${ }^{* * *}$ & $\begin{array}{l}\text { Second-order rate } \\
\text { constants with } \\
\text { biological molecules } \\
\mathbf{M}^{-1} \mathbf{s}^{-1}\end{array}$ \\
\hline Prostaglandins $\left(15 \mathrm{~d}-\mathrm{PGJ}_{2}\right)$ & $\begin{array}{l}\sim 2 \times 10^{-12} \mathrm{M} \text { of } 15 \mathrm{~d}-\mathrm{PGJ}_{2} \text { in } \\
3 \mathrm{~T} 3-\mathrm{LT} 1 \text { preadipocyte culture } \\
\text { medium }(96) \\
10^{-9} \mathrm{M} 15 \mathrm{~d}-\mathrm{PGJ}_{2} \text { intracellular } \\
(96)\end{array}$ & $\begin{array}{l}>1.8 \times 10^{5} \mathrm{~s} \text { in aqueous } \\
\text { media at } 37^{\circ} \mathrm{C} \text { for } 15 \mathrm{~d}- \\
\mathrm{PGJ}_{2}(96)\end{array}$ & Not determined & $\begin{array}{l}\mathrm{PGJ}_{2} \sim 0.7 \mathrm{M}^{-1} \mathrm{~s}^{-1} \text { for } \\
\mathrm{GSH} \text { with } 15 \mathrm{~d}-\mathrm{PGJ}_{2} \text { at } \\
\mathrm{pH} 7.4,37^{\circ} \mathrm{C}(95)\end{array}$ \\
\hline
\end{tabular}

Half-life: The time taken for the initial concentration of a reactive species to be reduced by $50 \%$.

Diffusion distance: The distance travelled by a reactive species before $90 \%$ is consumed by a chemical process. The half-life and diffusion distance of a reactive species are interrelated (see section 3.3). The half-life (and thus the diffusion distance) is also dependent on the identity and concentration of substrates that lie in the path of the reactive species. Where applicable, we have specified the medium (buffer/plasma/in cell) in which the half-lives and the diffusion distances were calculated. See section 3.3 and cited references therein for more detailed discussion. 


\section{Table 2}

A selection of cellular ROS and RES scavengers, their intracellular concentrations, and reaction kinetics (with $\mathrm{pH}$ and temperature at which the rates were determined given when possible) with various RES/ROS.

\begin{tabular}{|c|c|c|}
\hline RES/ROS scavengers & Concentration range & Reaction rates with RES/ROS \\
\hline GSH & $1-10 \mathrm{mM}(97,98)$ & See Table 1 \\
\hline Carnosine & $150 \mathrm{mM}(99)$ & $\begin{array}{l}\text { HNE }\left(0.0021 \mathrm{M}^{-1} \mathrm{~s}^{-1}\right) \\
\text { ONE }\left(0.022 \mathrm{M}^{-1} \mathrm{~s}^{-1}\right) \\
\text { both at } \mathrm{pH} 7.4,37^{\circ} \mathrm{C}(100)\end{array}$ \\
\hline SOD & $\begin{array}{l}24 \mu \mathrm{M} \mathrm{Cu}-\mathrm{Zn} \text { SOD in rat liver cytosol;(101) } 11 \\
\mu \mathrm{M} \mathrm{Mn-SOD} \text { in rat liver mitochondria;(101) } 20- \\
90 \mathrm{U} / \mathrm{mg} \text { in most cell lines of total SOD } \\
\text { activity(102) }\end{array}$ & $\begin{array}{l}\text { Diffusion controlled reaction with superoxide }\left(10^{9} \mathrm{M}^{-1} \mathrm{~s}^{-1}\right) \text { at } \\
\text { pH } 7.8(103) \\
\text { Also possesses peroxidase activity }\left(1600 \mathrm{M}^{-1} \mathrm{~s}^{-1} \text { for } \mathrm{HO}_{2} \mathrm{CO}_{2}^{-}\right. \\
\left.\text {at } \mathrm{pH} 7.4,25^{\circ} \mathrm{C}\right)(104)\end{array}$ \\
\hline peroxiredoxins & $\begin{array}{l}\text { Peroxiredoxin1 and } 2(\text { Cytosol; } 65 \mu \mathrm{M}) ;(105) \\
\text { Peroxiredoxin3 (mitochondria; } 125 \mu \mathrm{M})(105)\end{array}$ & $\begin{array}{l}\text { React with } \mathrm{ONOO}^{-} \text {at } 10^{6}-10^{8} \mathrm{M}^{-1} \mathrm{~s}^{-1} \text { at } \mathrm{pH} 7.4,25^{\circ} \mathrm{C} \\
(106,107) \\
\text { React with } \mathrm{H}_{2} \mathrm{O}_{2} \text { at } 10^{5}-10^{8} \mathrm{M}^{-1} \mathrm{~s}^{-1} \text { at } \mathrm{pH} 7.4,20^{\circ} \mathrm{C}(108-111)\end{array}$ \\
\hline Catalase & $\sim 10^{5} \mathrm{U} / \mathrm{mg}$ of protein $(102)$ & Reacts with $\mathrm{H}_{2} \mathrm{O}_{2}$ at $10^{7} \mathrm{M}^{-1} \mathrm{~s}^{-1}$ at $\mathrm{pH} 7.4,20^{\circ} \mathrm{C}(108,112)$ \\
\hline Glutathione peroxidase & $\begin{array}{l}\text { 14-30 U/mg of protein }(102) \\
\sim 2 \mu \mathrm{M} \text { in cells }(112)\end{array}$ & Reacts with $\mathrm{H}_{2} \mathrm{O}_{2}$ at $10^{7}-10^{8} \mathrm{M}^{-1} \mathrm{~s}^{-1}$ at $\mathrm{pH} 7.4,20^{\circ} \mathrm{C}(108,112)$ \\
\hline
\end{tabular}




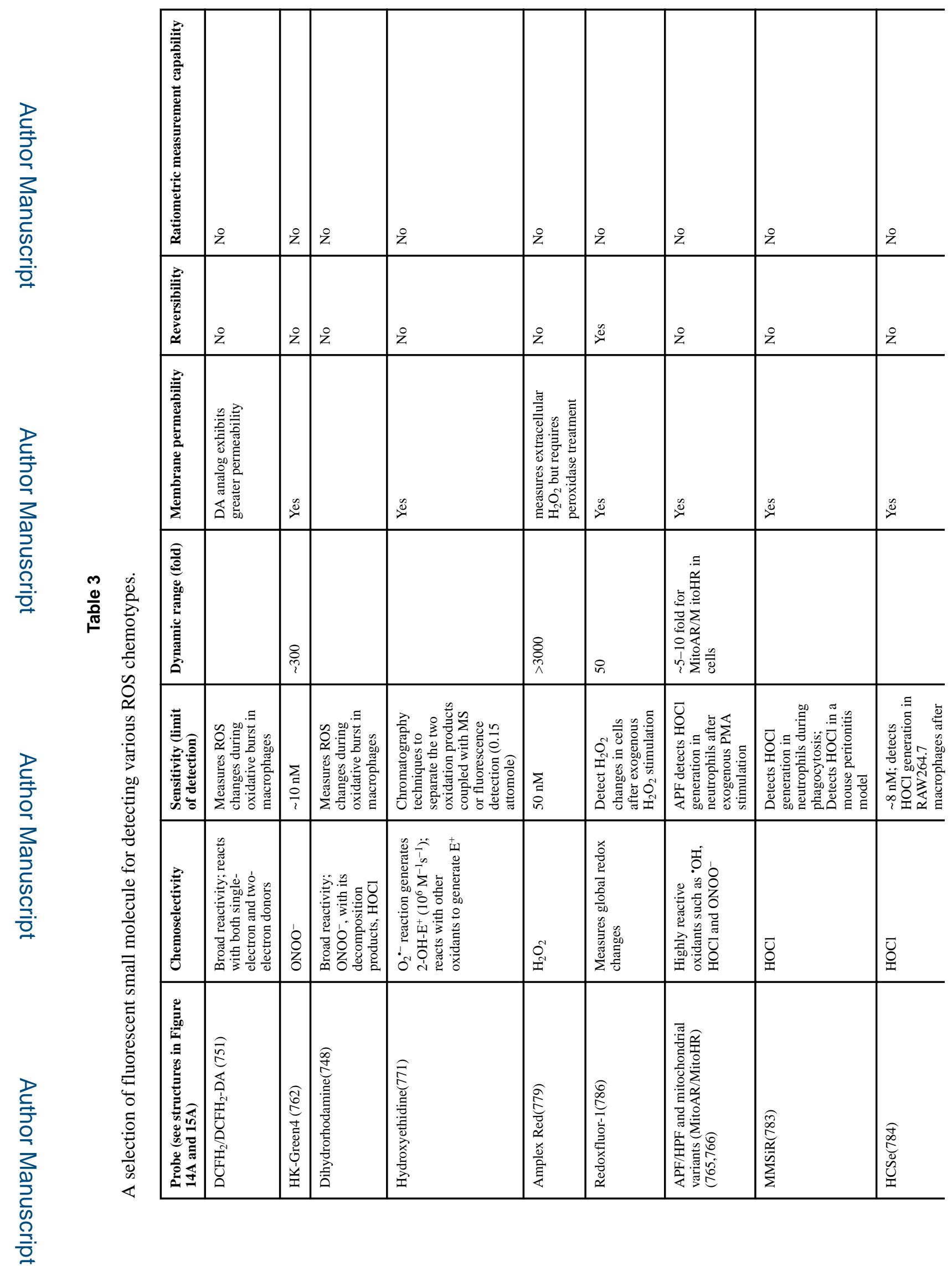

Chem Rev. Author manuscript; available in PMC 2019 September 26. 


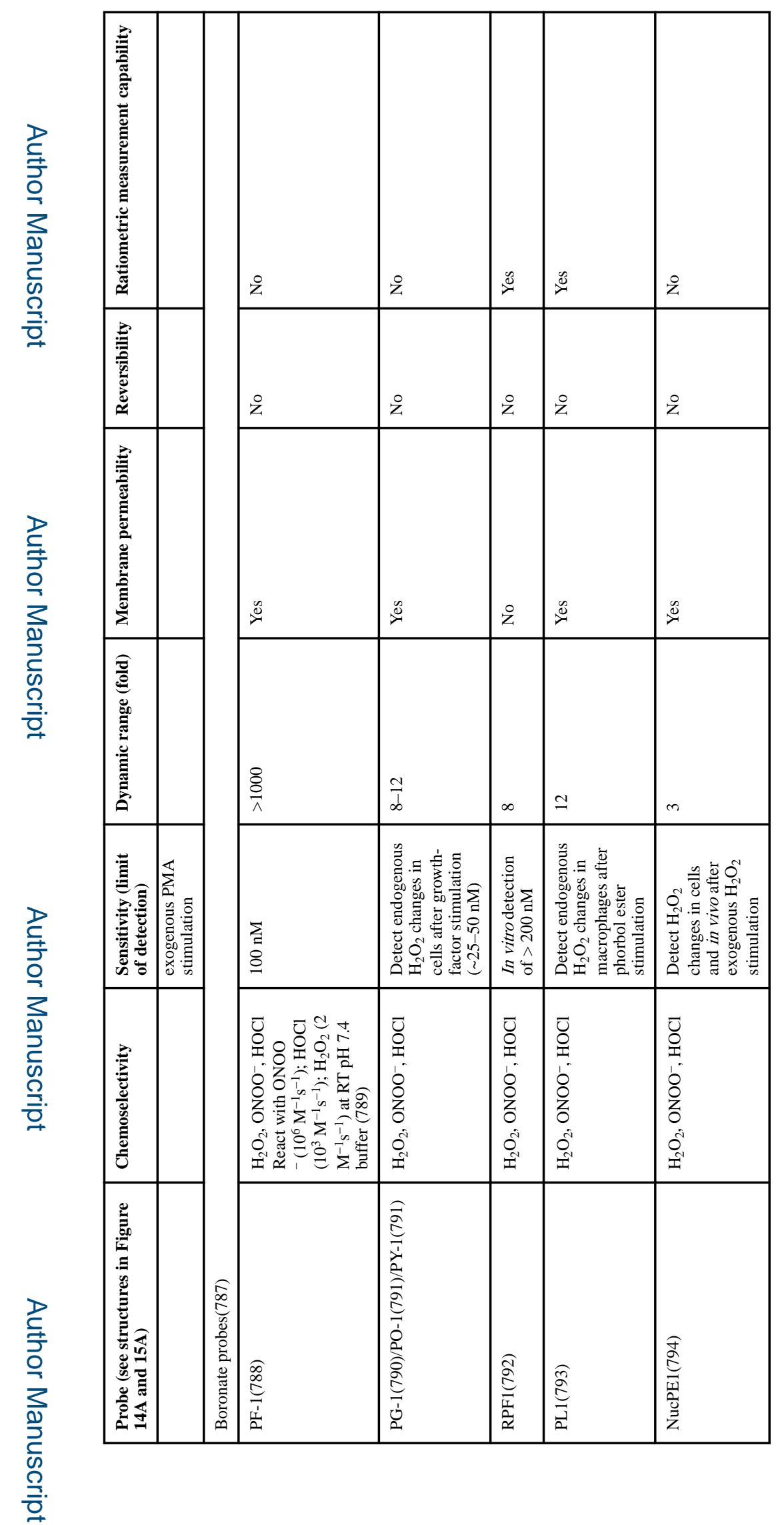

Chem Rev. Author manuscript; available in PMC 2019 September 26. 


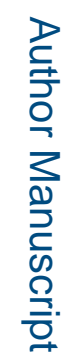

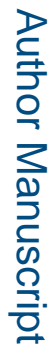

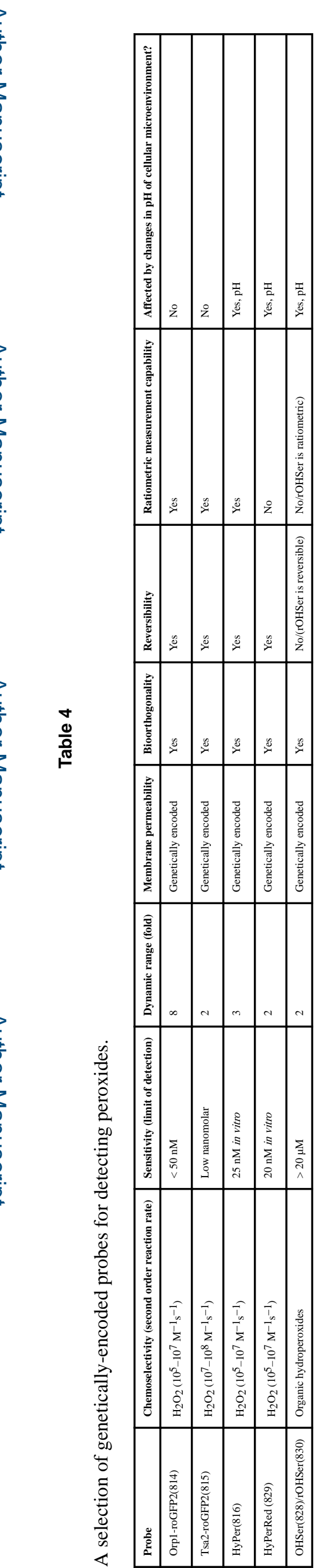

ב

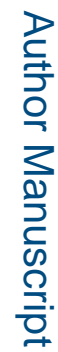

